Scaffold10141_498901-498975(+) mir-203

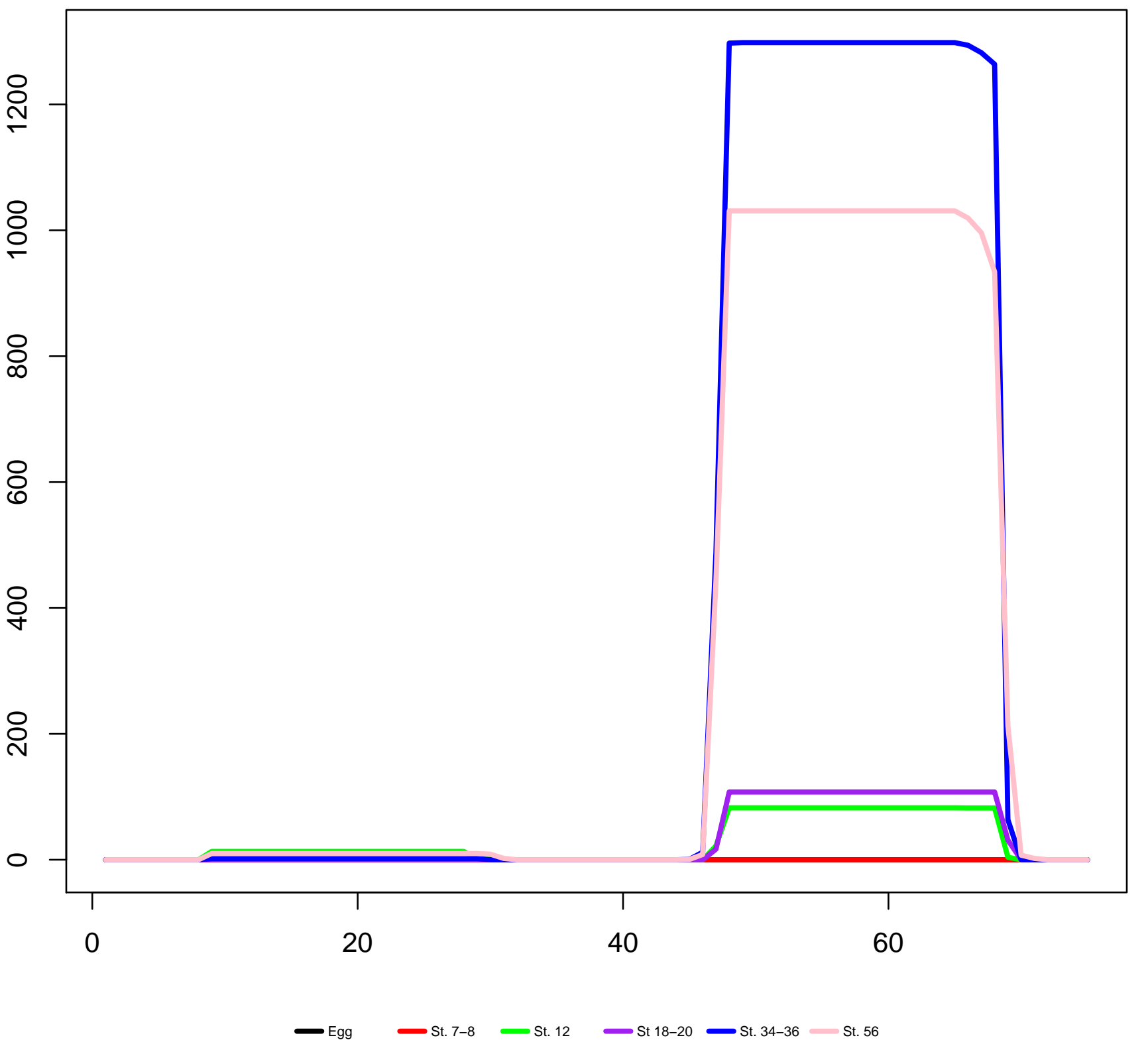


Scaffold101411_31235-31296(+) mir-1b

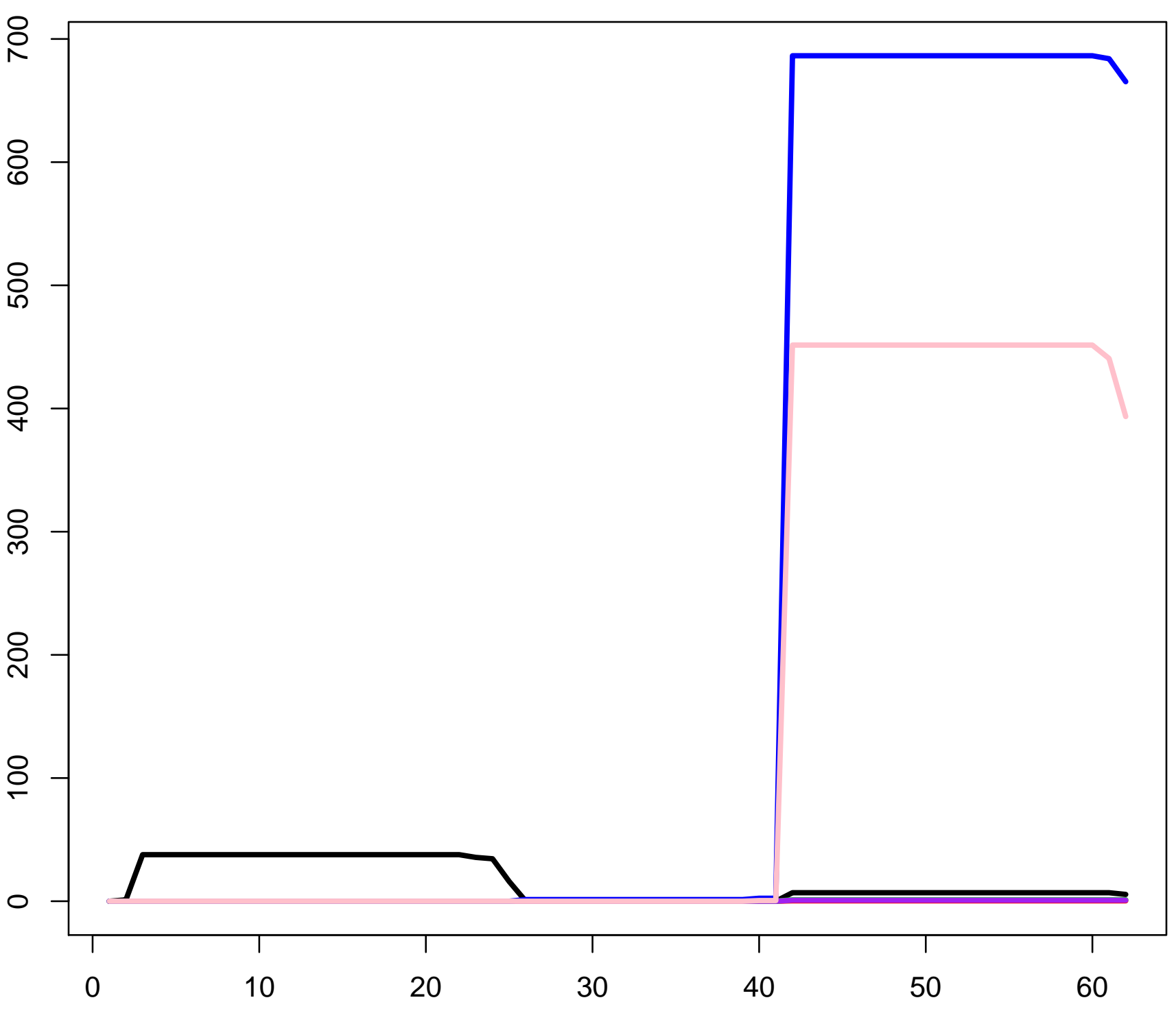


Scaffold101411_31647-31721(+) mir-133d

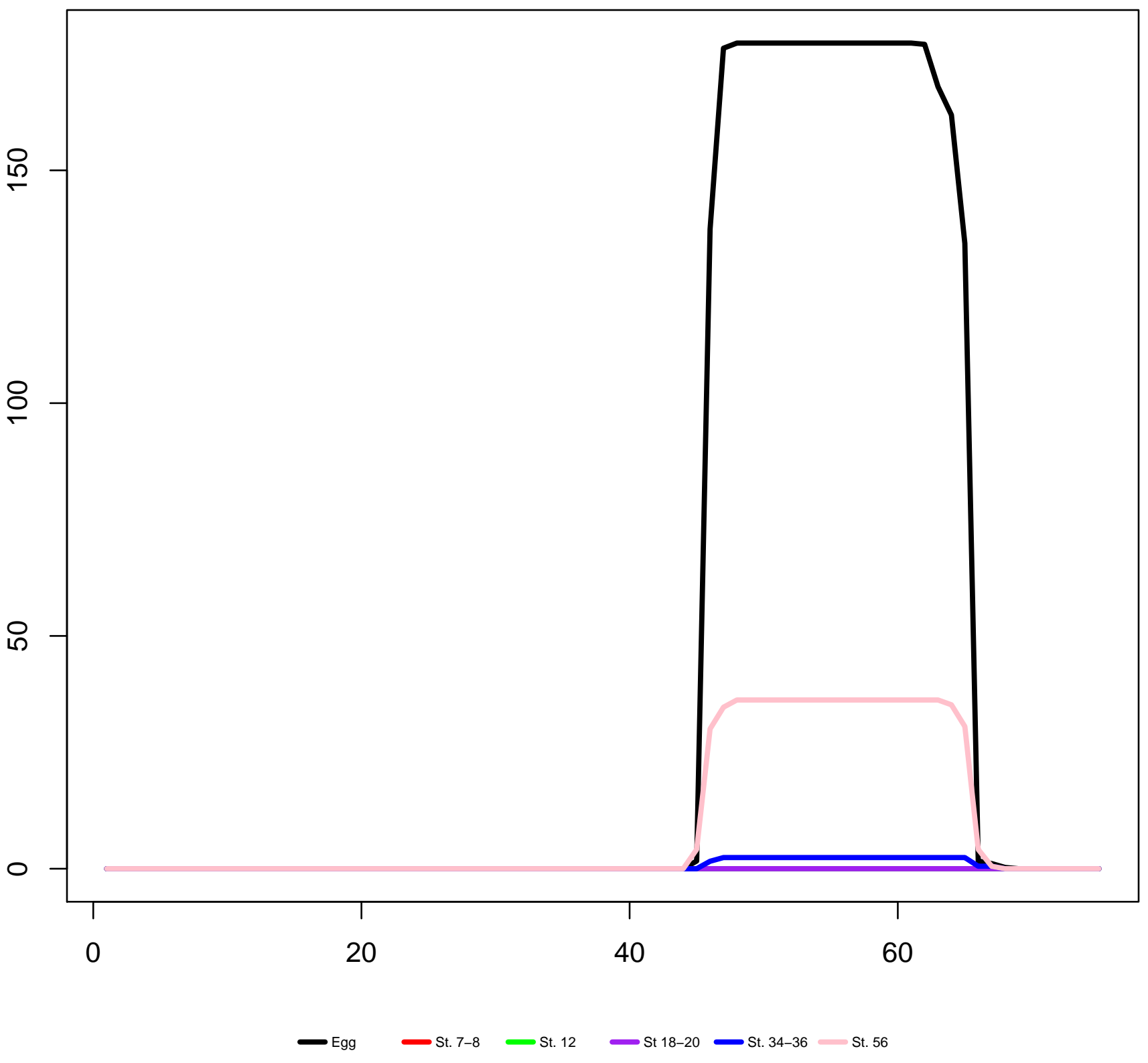


Scaffold10218_605312-605388(-) mir-30b

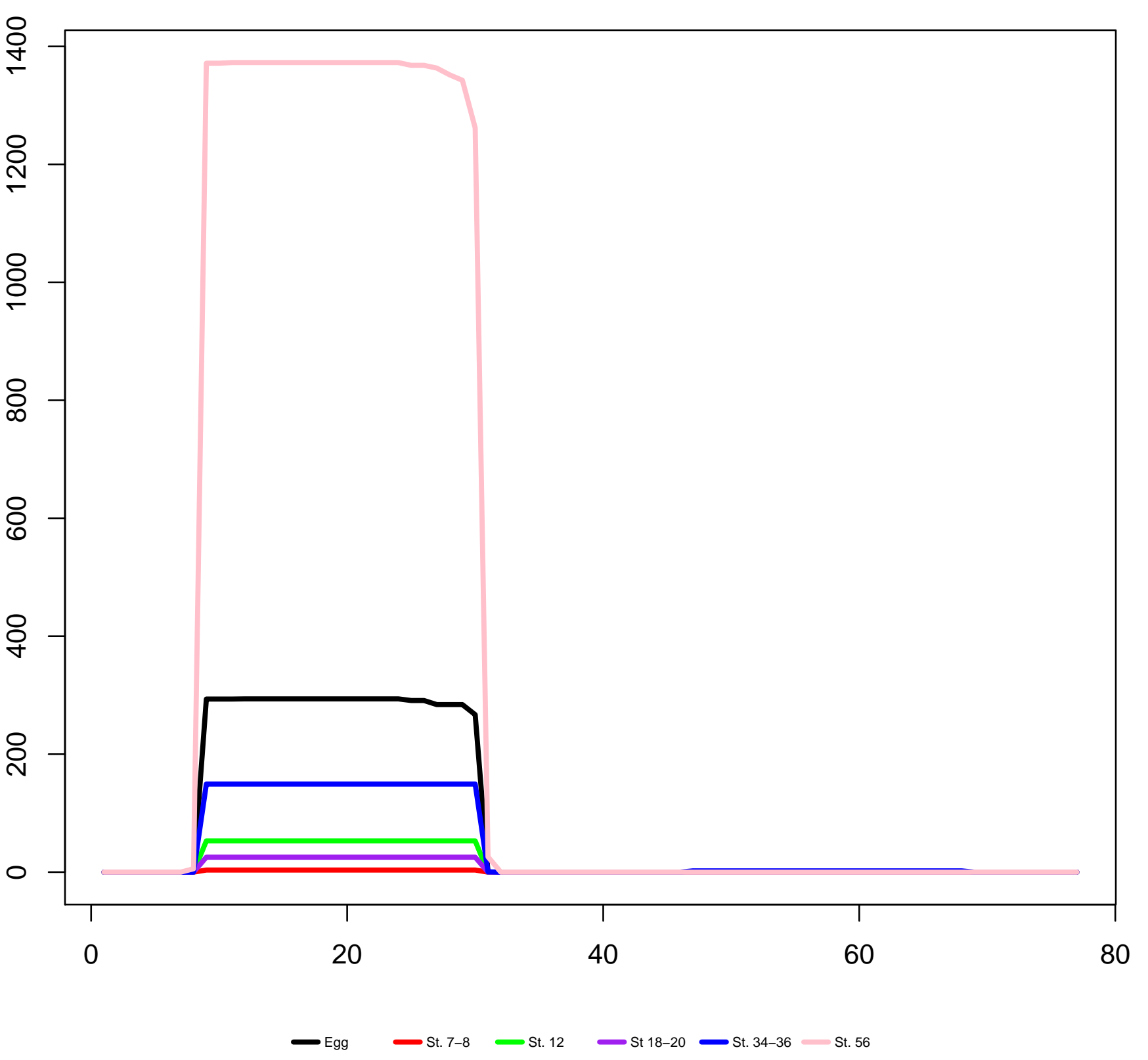


Scaffold102692_237773-237853(-) mir-128-1

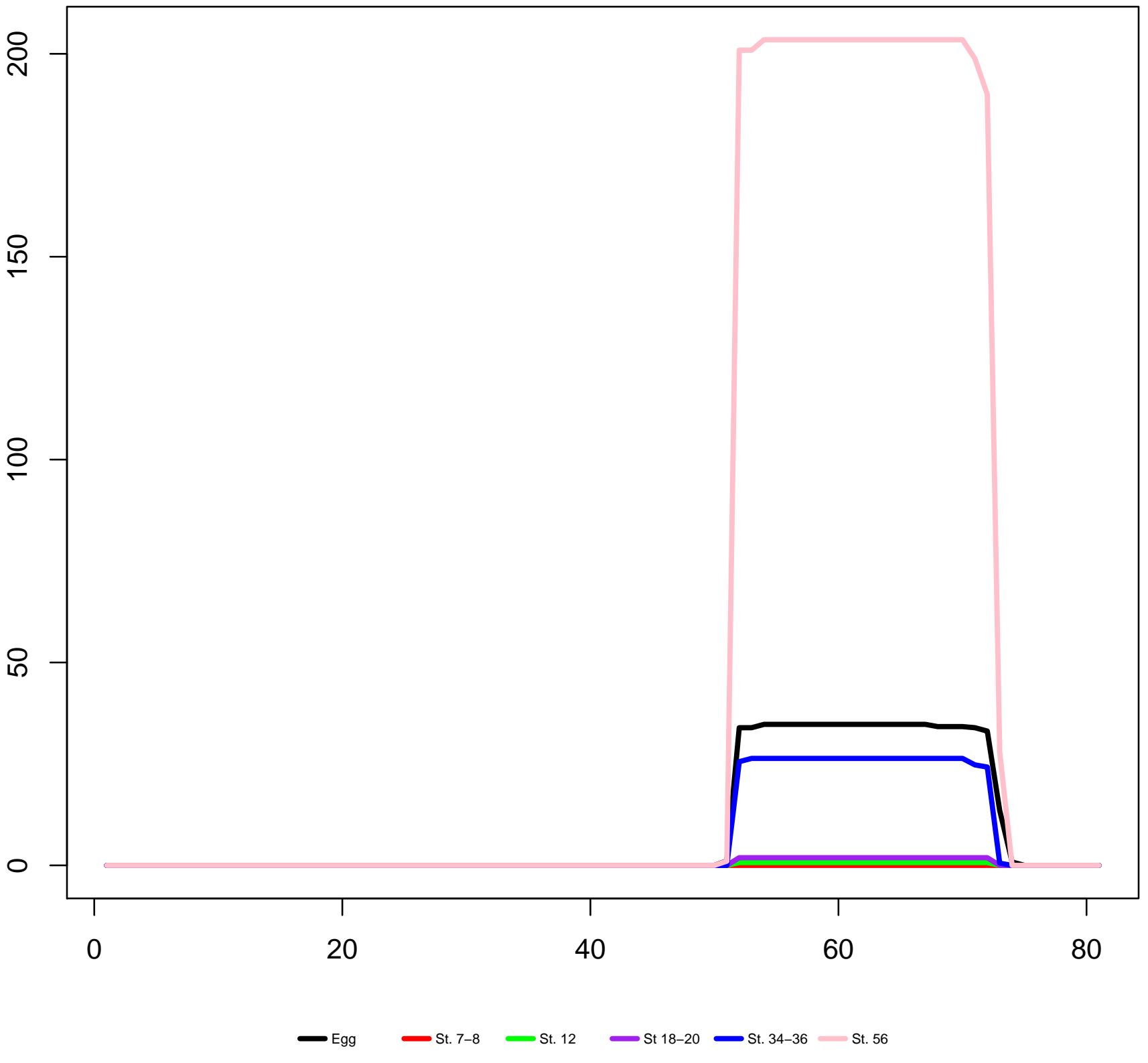


Scaffold105945_612251-612341(+) mir-101-2

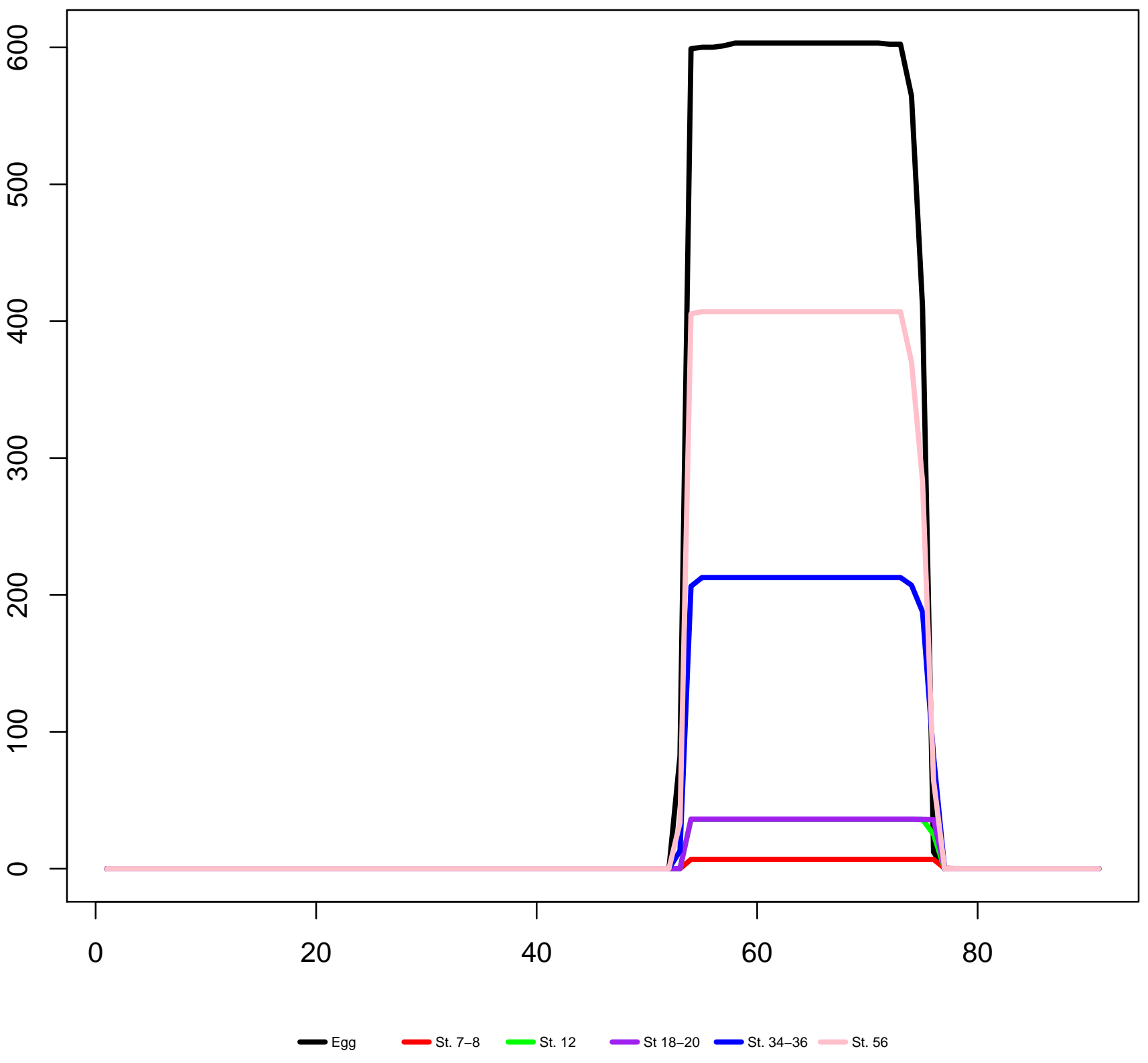


Scaffold10627_2149498-2149586(+) mir-218-2

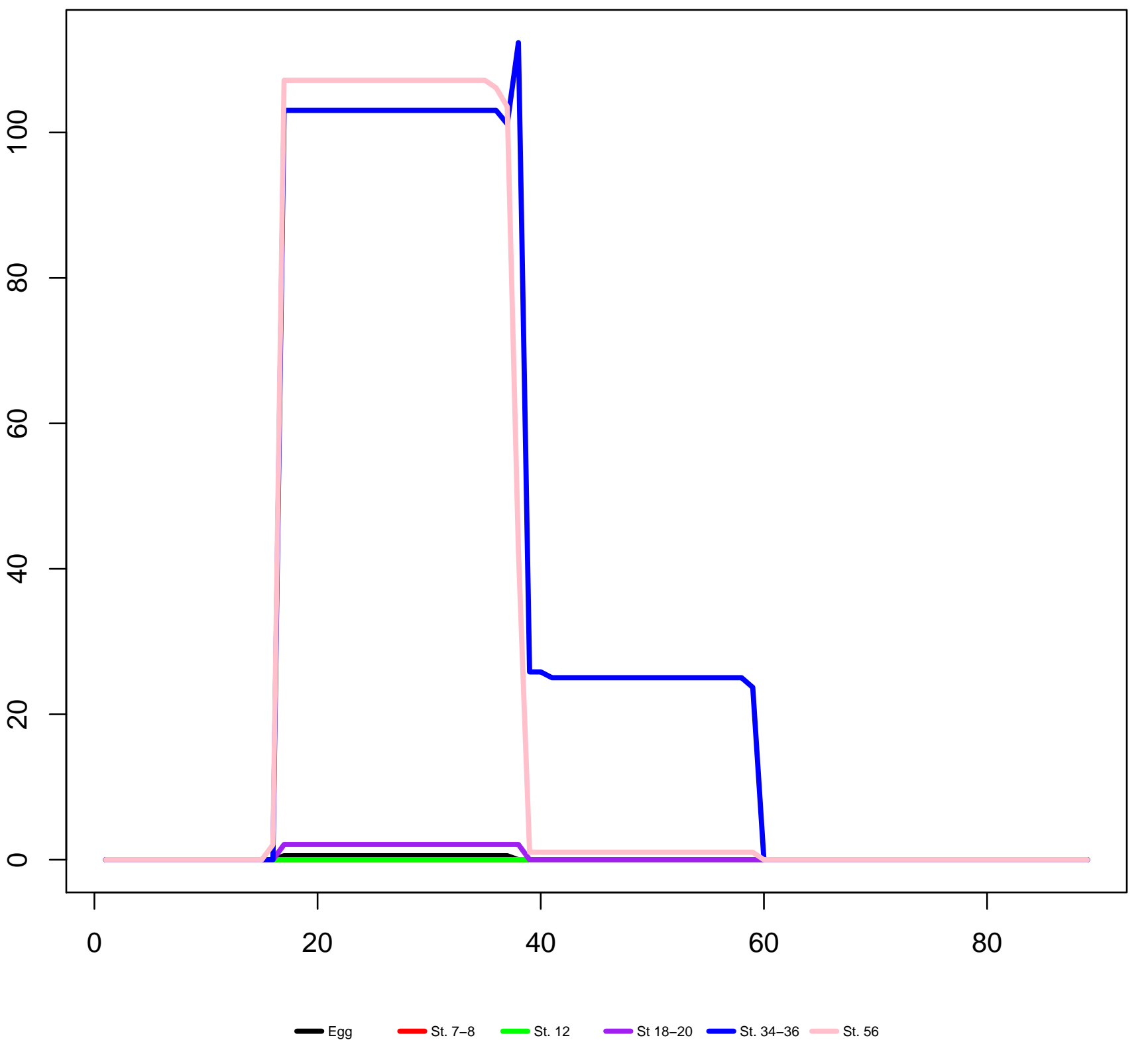


Scaffold10791_1087685-1087759(-) mir-338-3

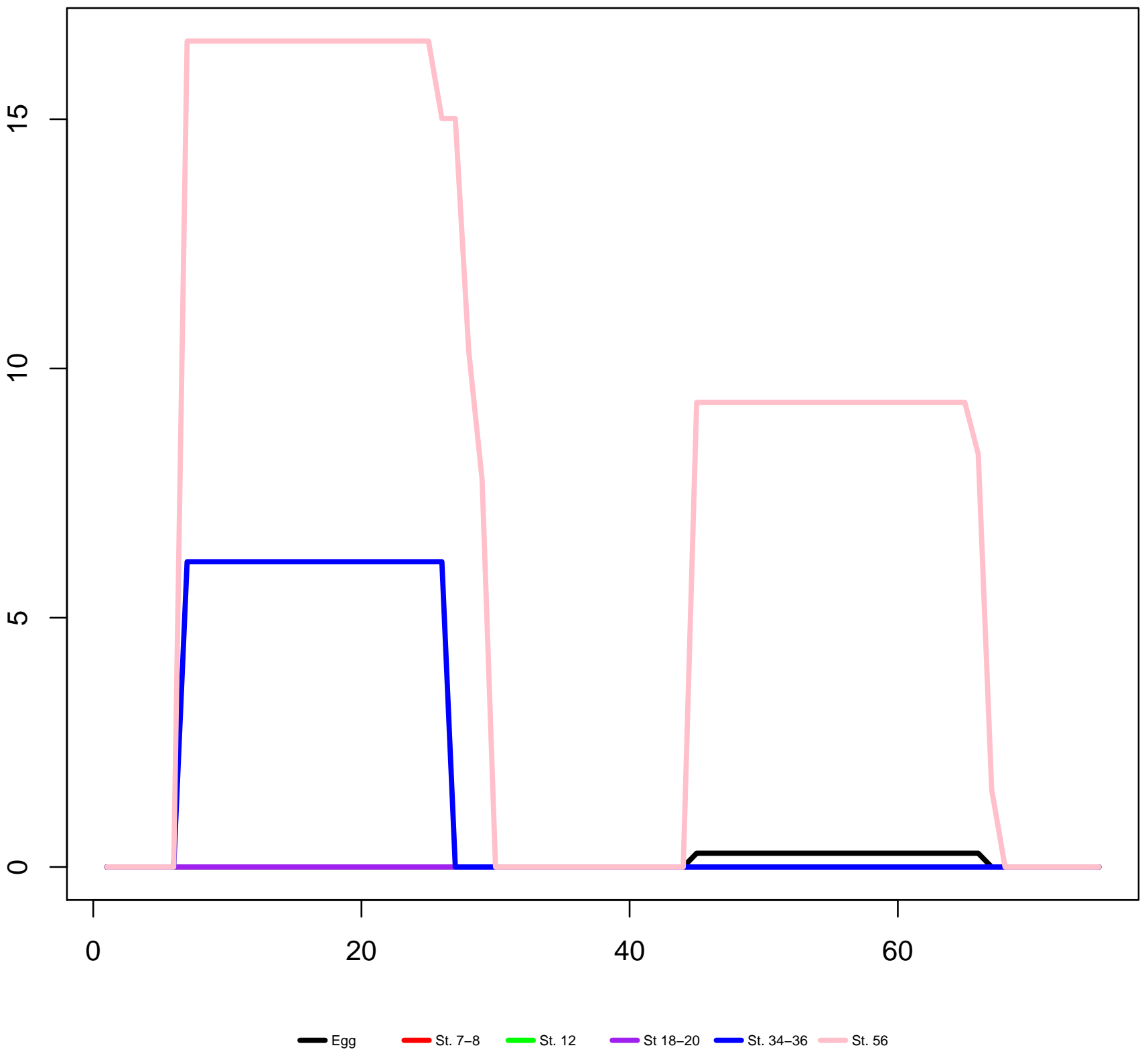


Scaffold10835_65585-65672(-) mir-196-2

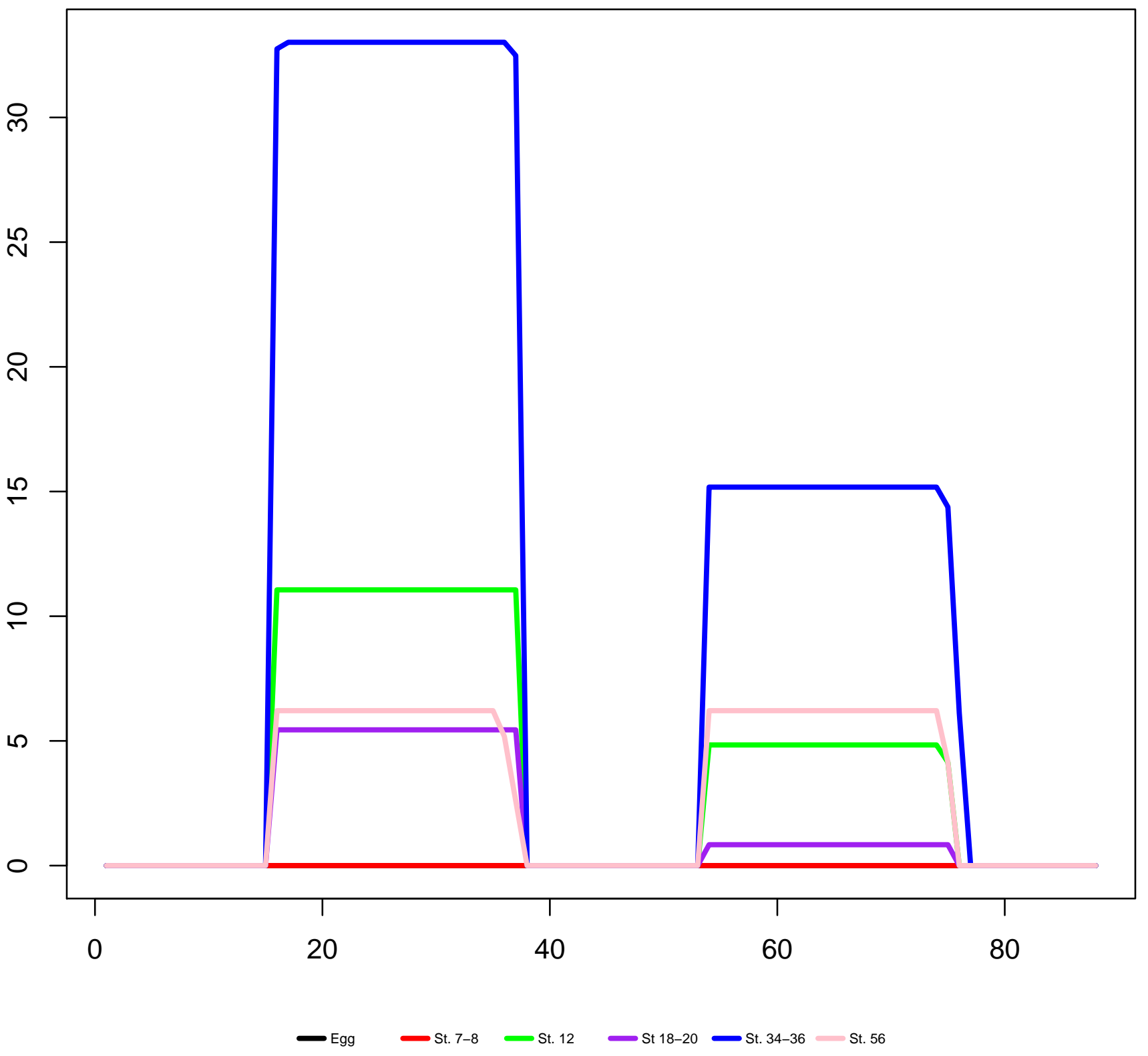


Scaffold10870_213552-213633(-) mir-129

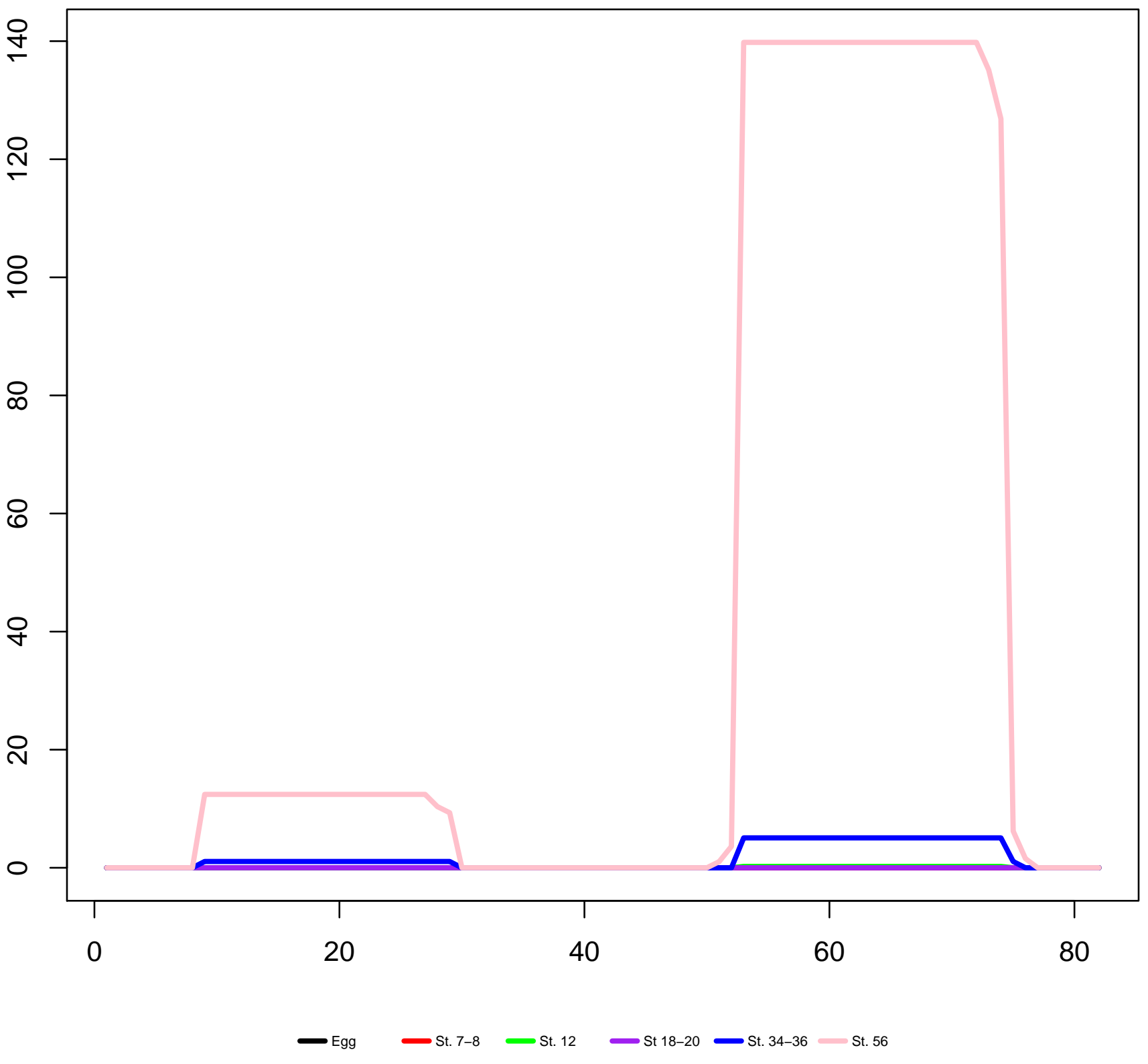


Scaffold118305_589175-589249(+) mir-26b

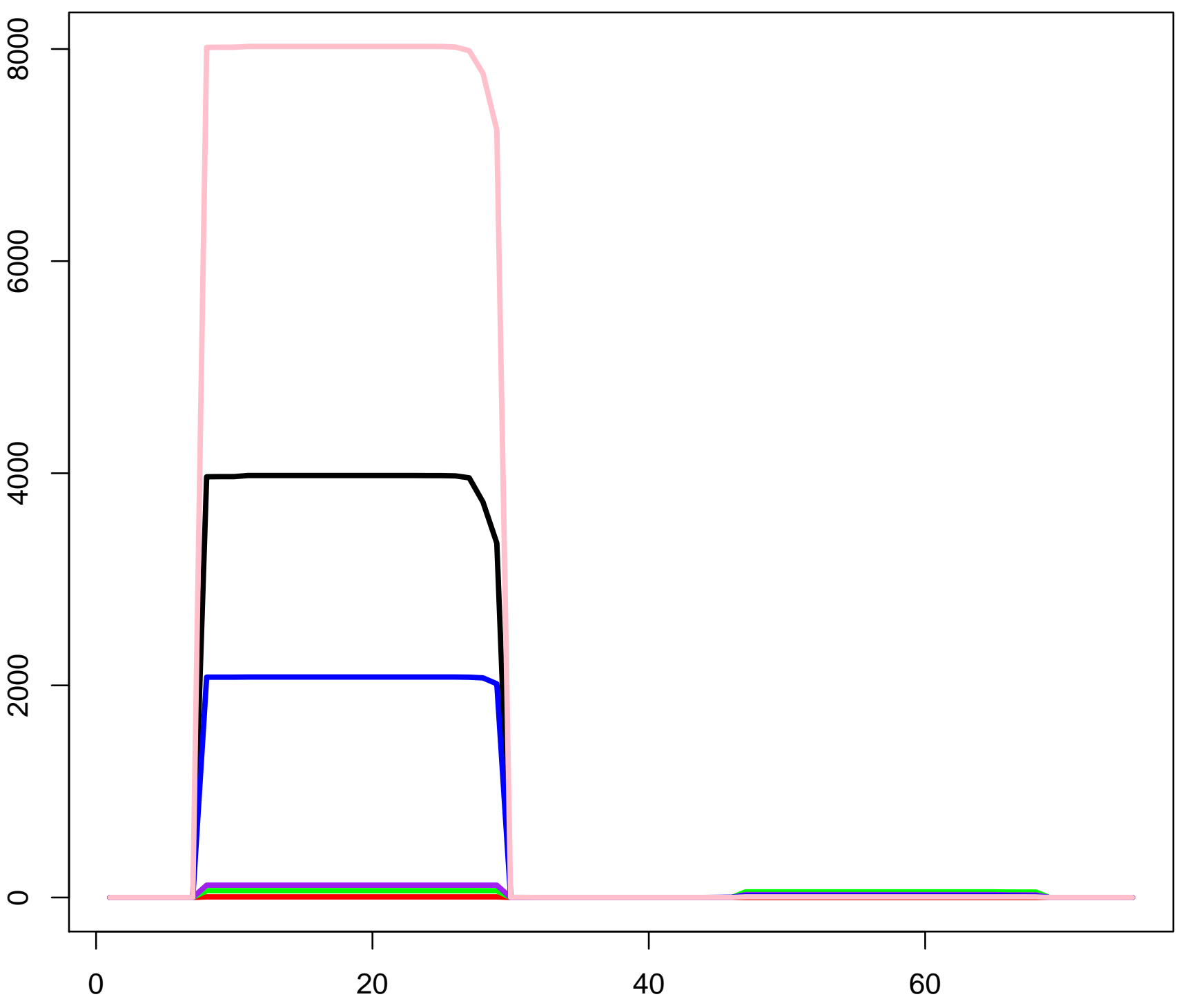


Scaffold12053_196336-196452(-) mir-7-2

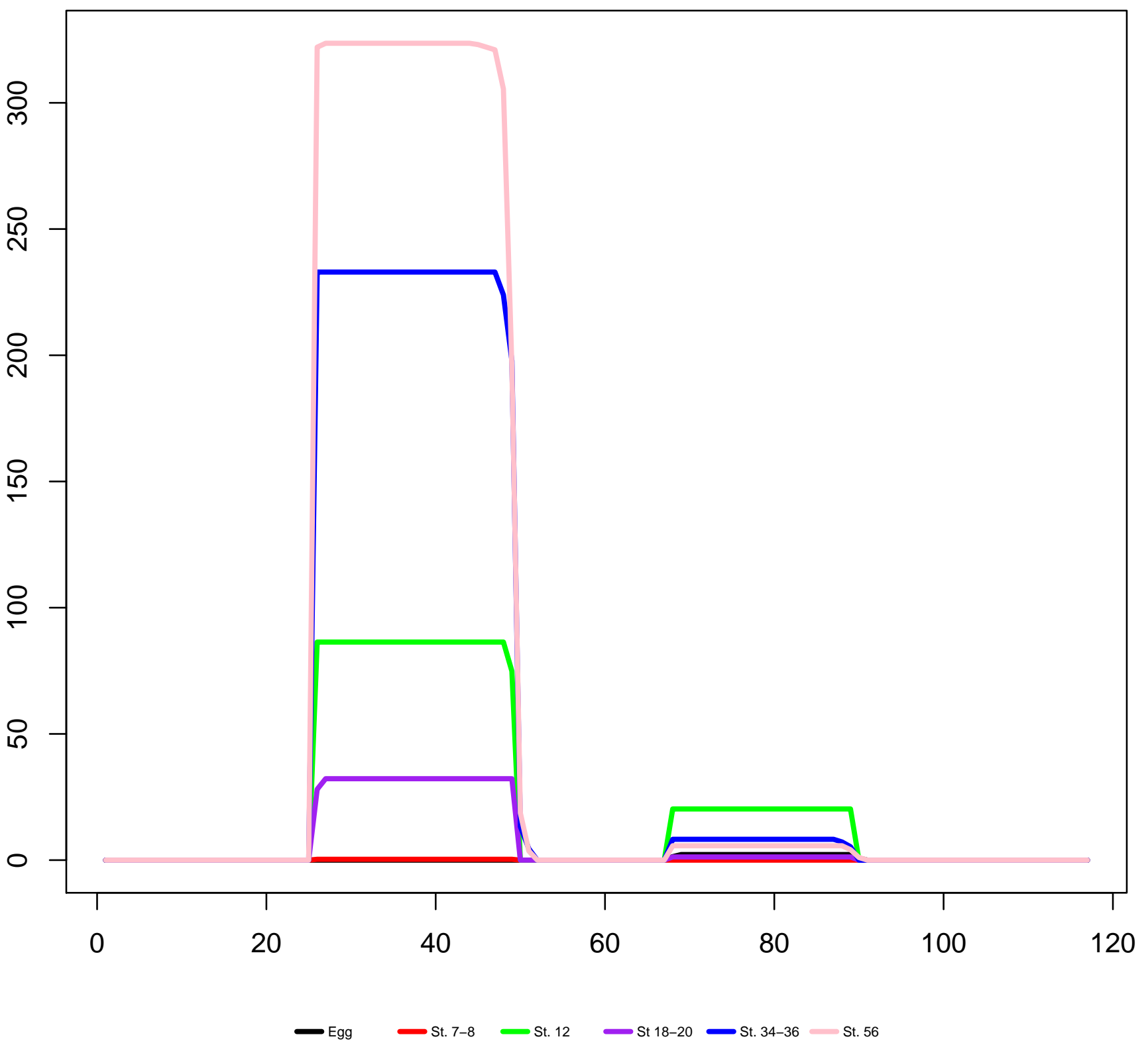


Scaffold12053_1652945-1653042(+) mir-27b

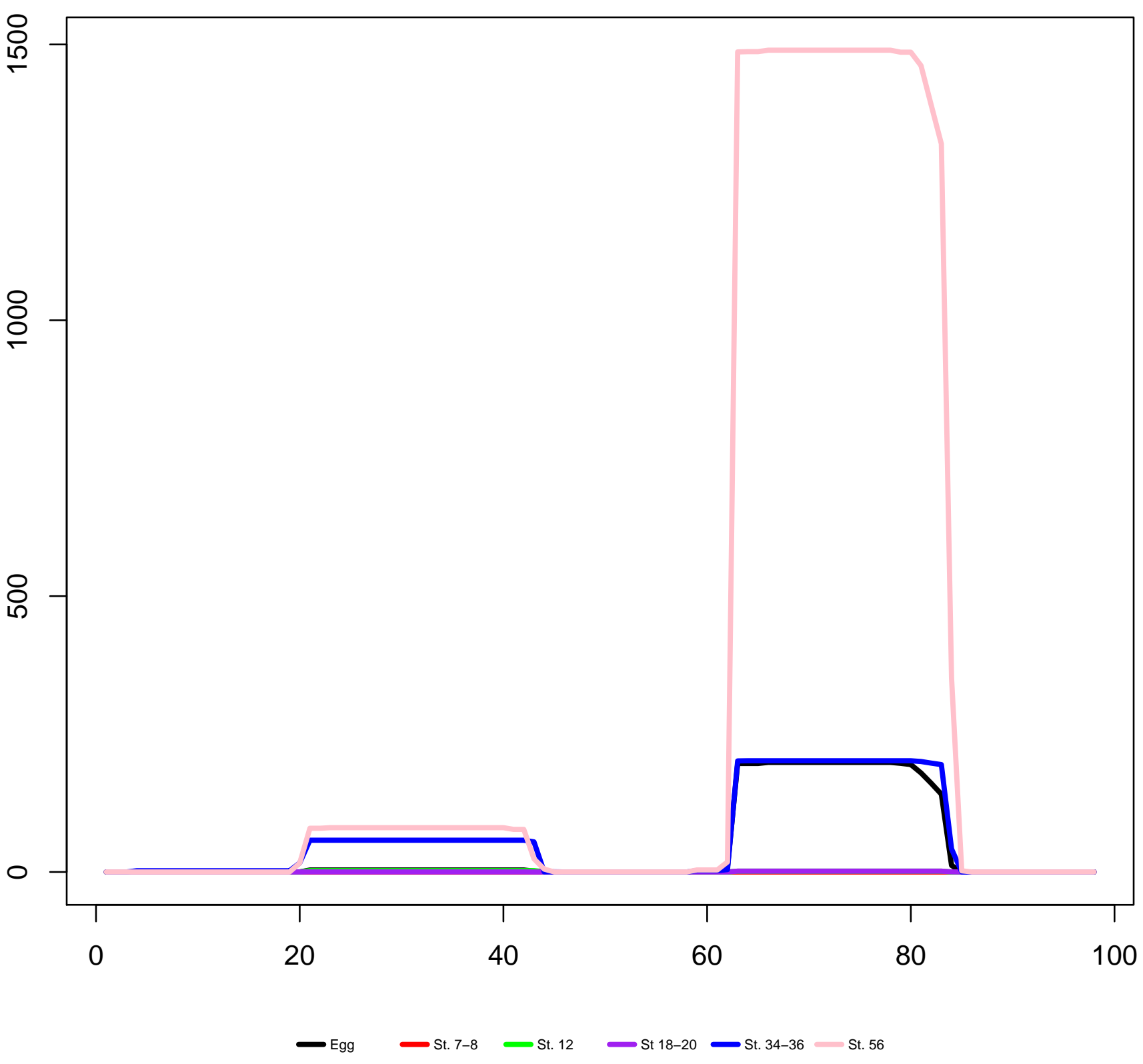


Scaffold12407_362879-362945(+) mir-302

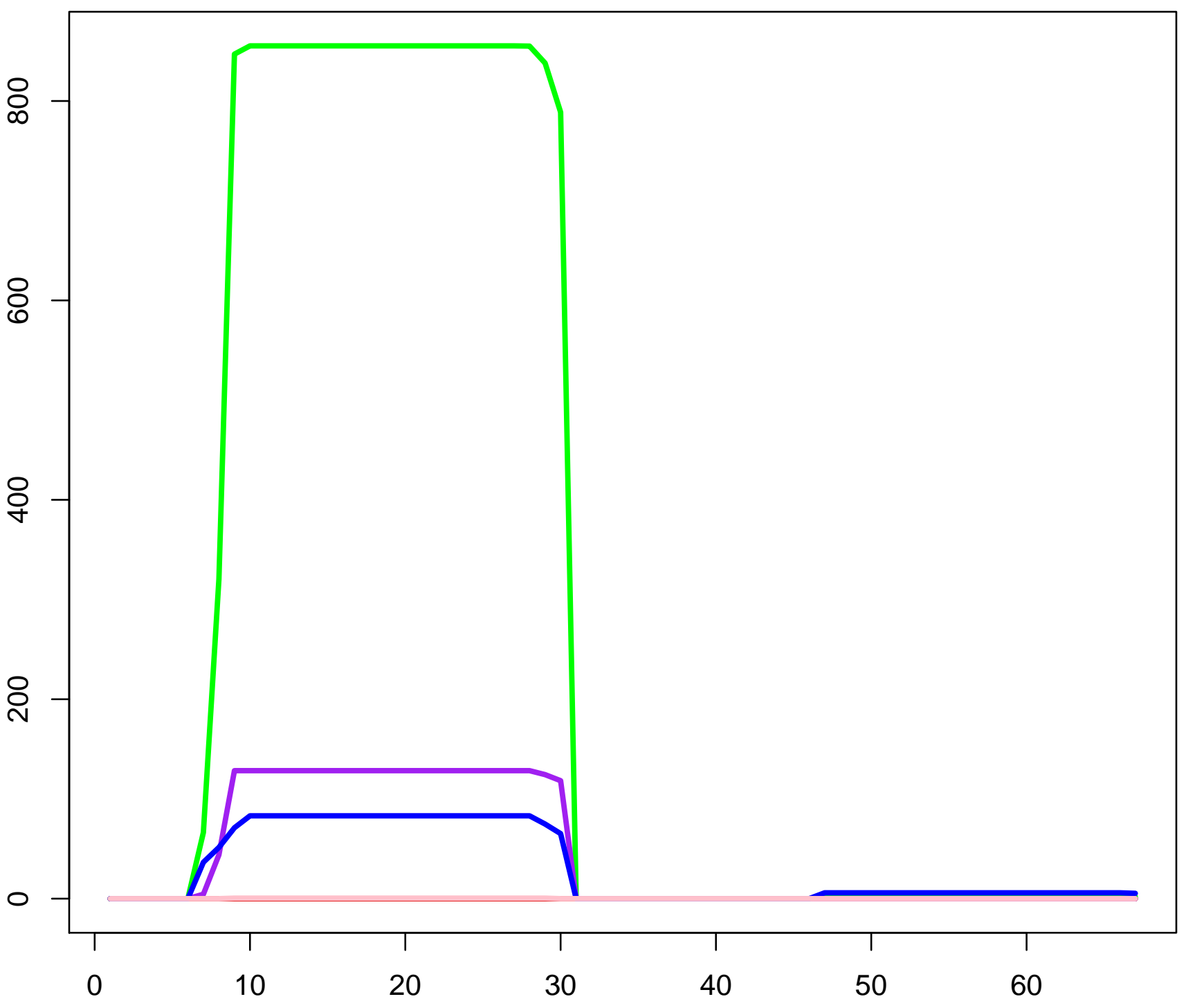


Scaffold12424_18929-18998(-) mir-19b

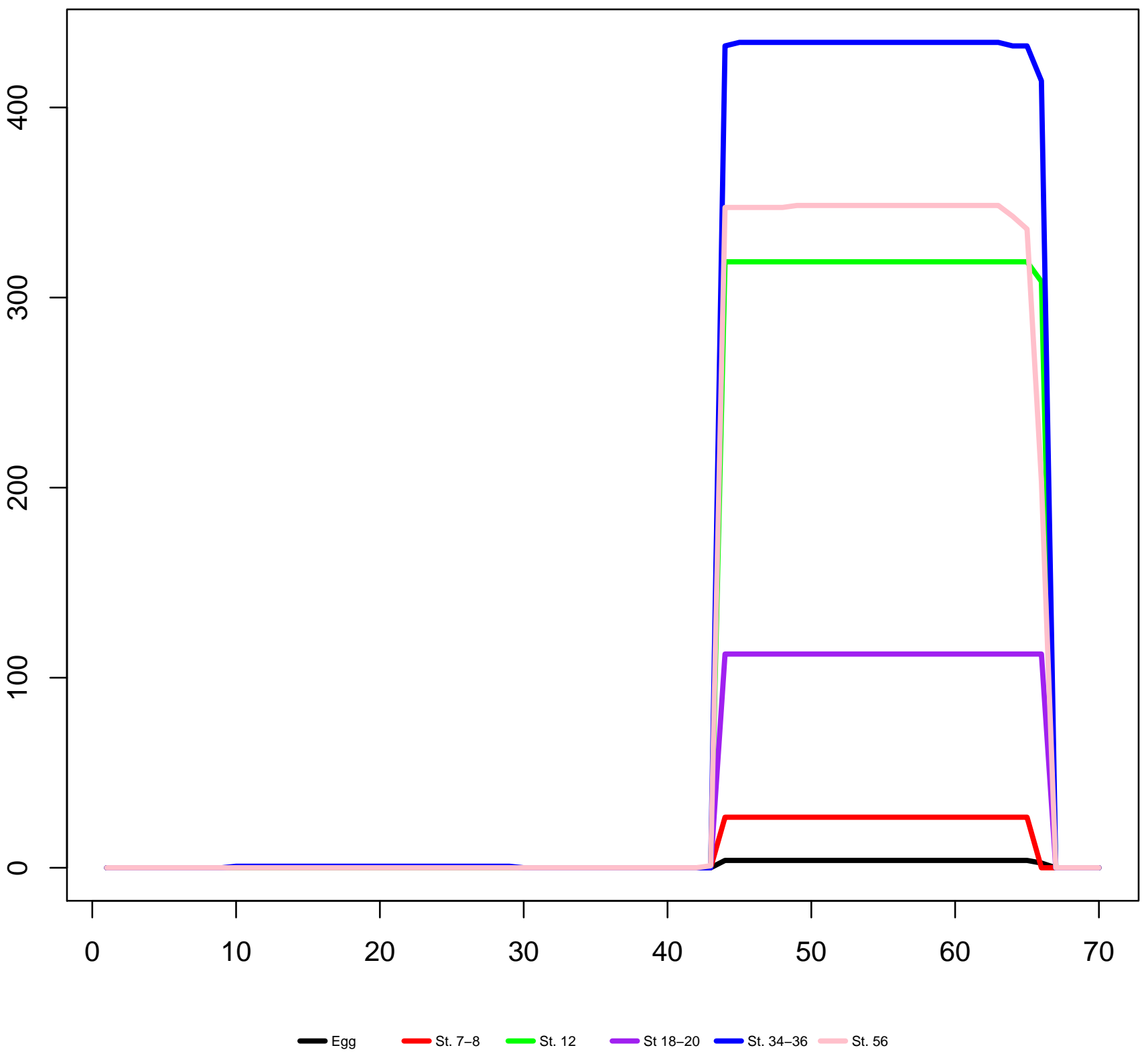


Scaffold12424_19327-19389(-) mir-106

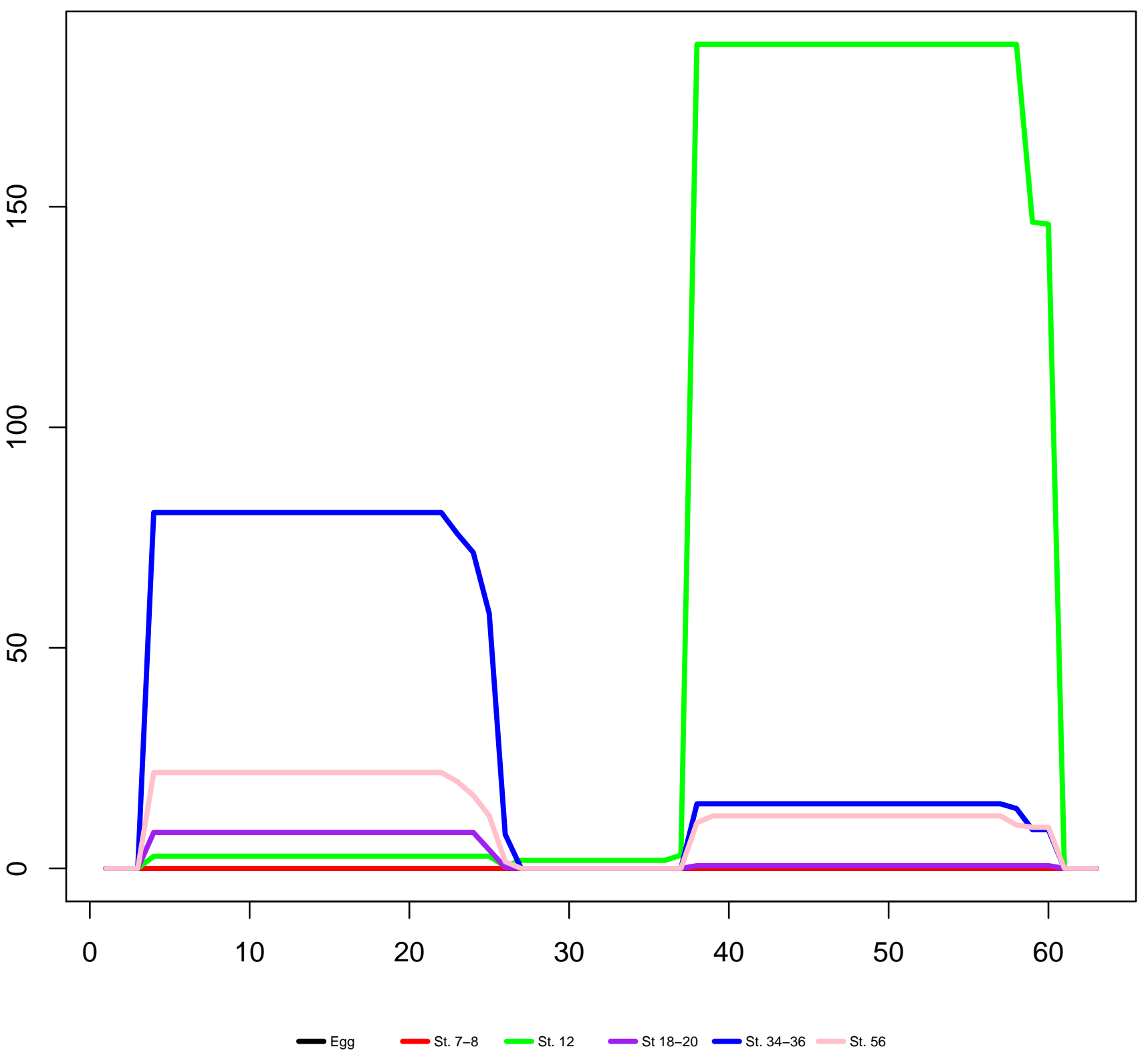


Scaffold12526_351541-351635(-) mir-204a-2

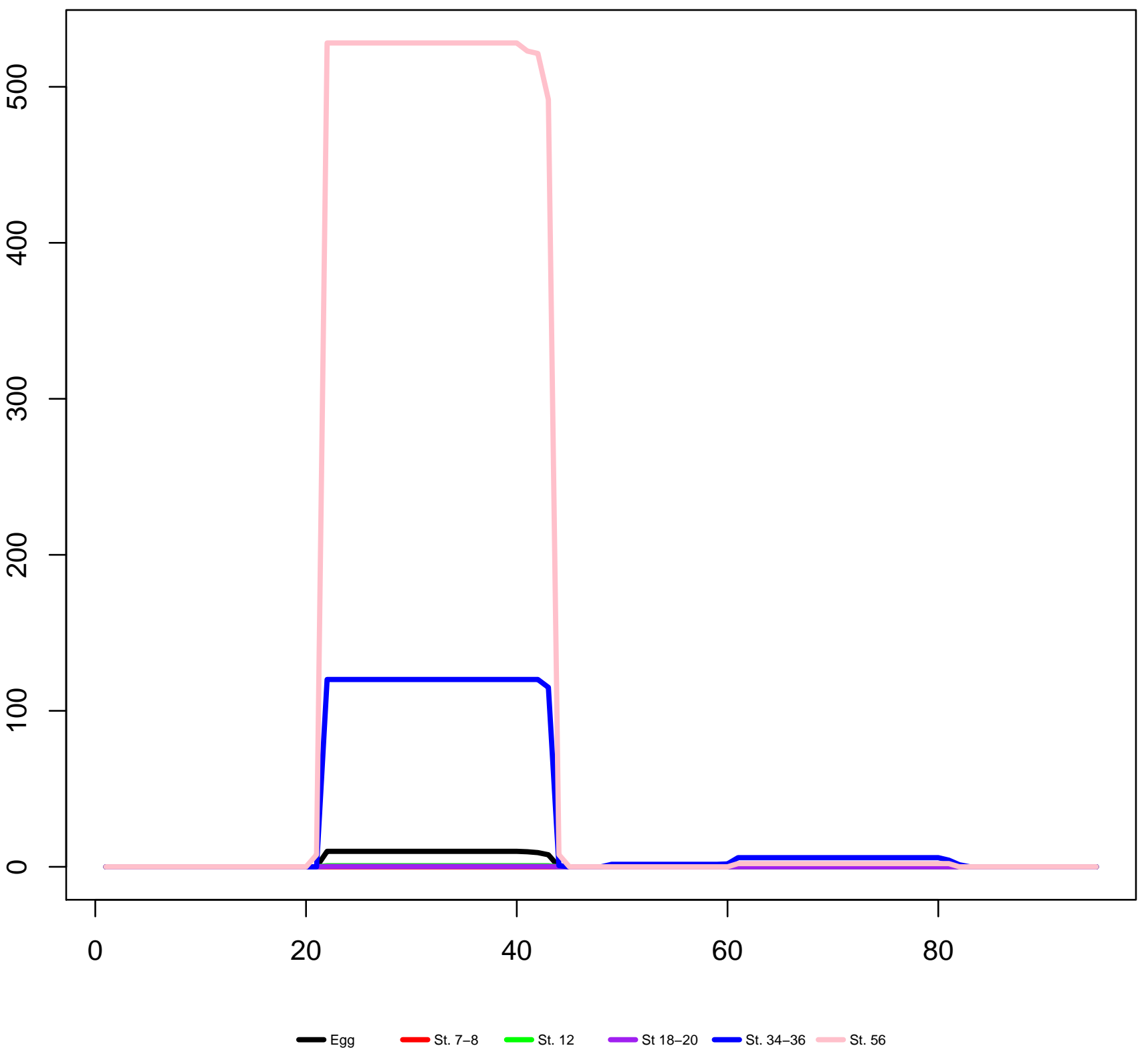


Scaffold131793_839153-839245(+) mir-1329

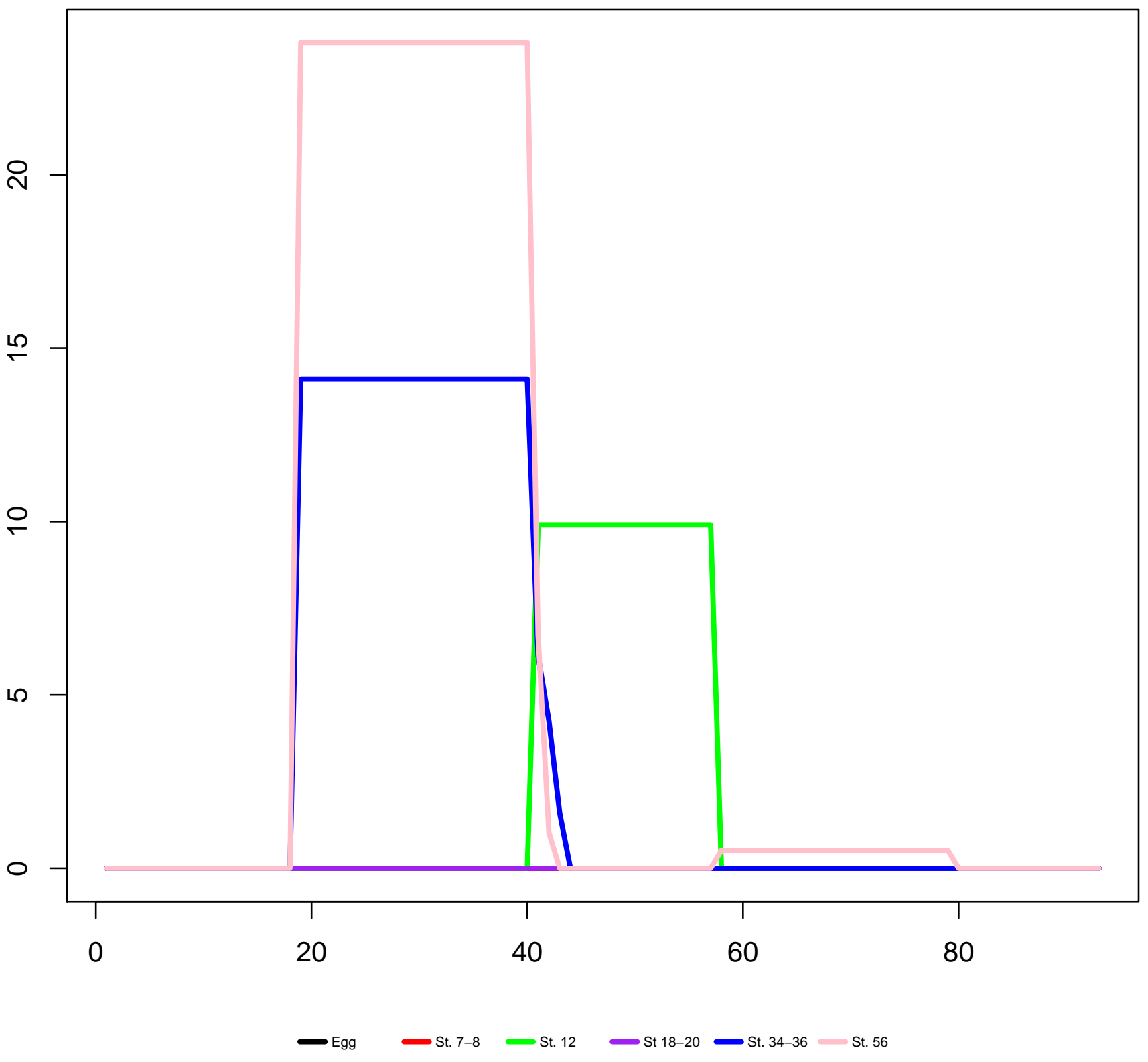


Scaffold133533_1-99(-) mir-153-1

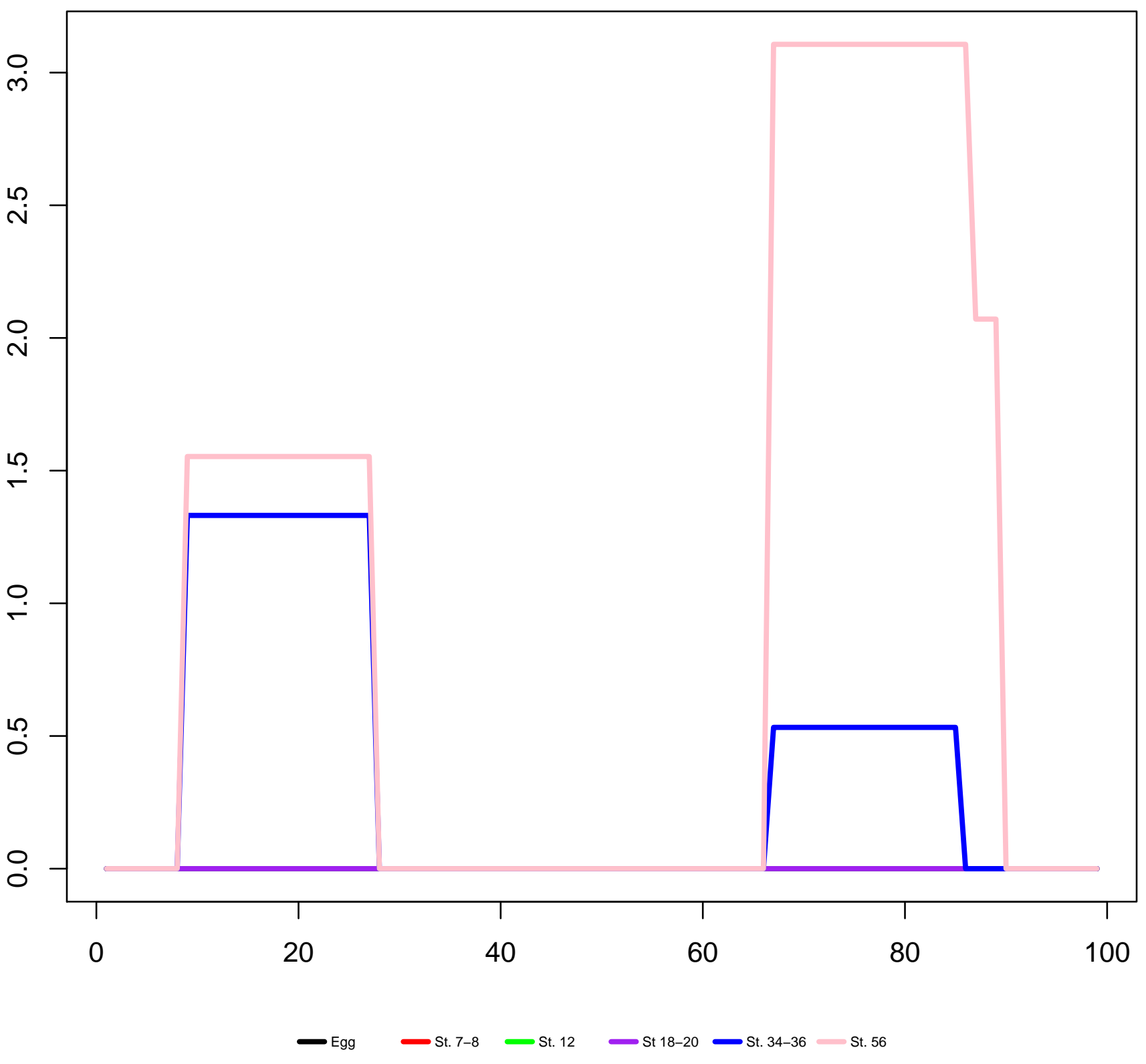


Scaffold134640_14-105(-) mir-455

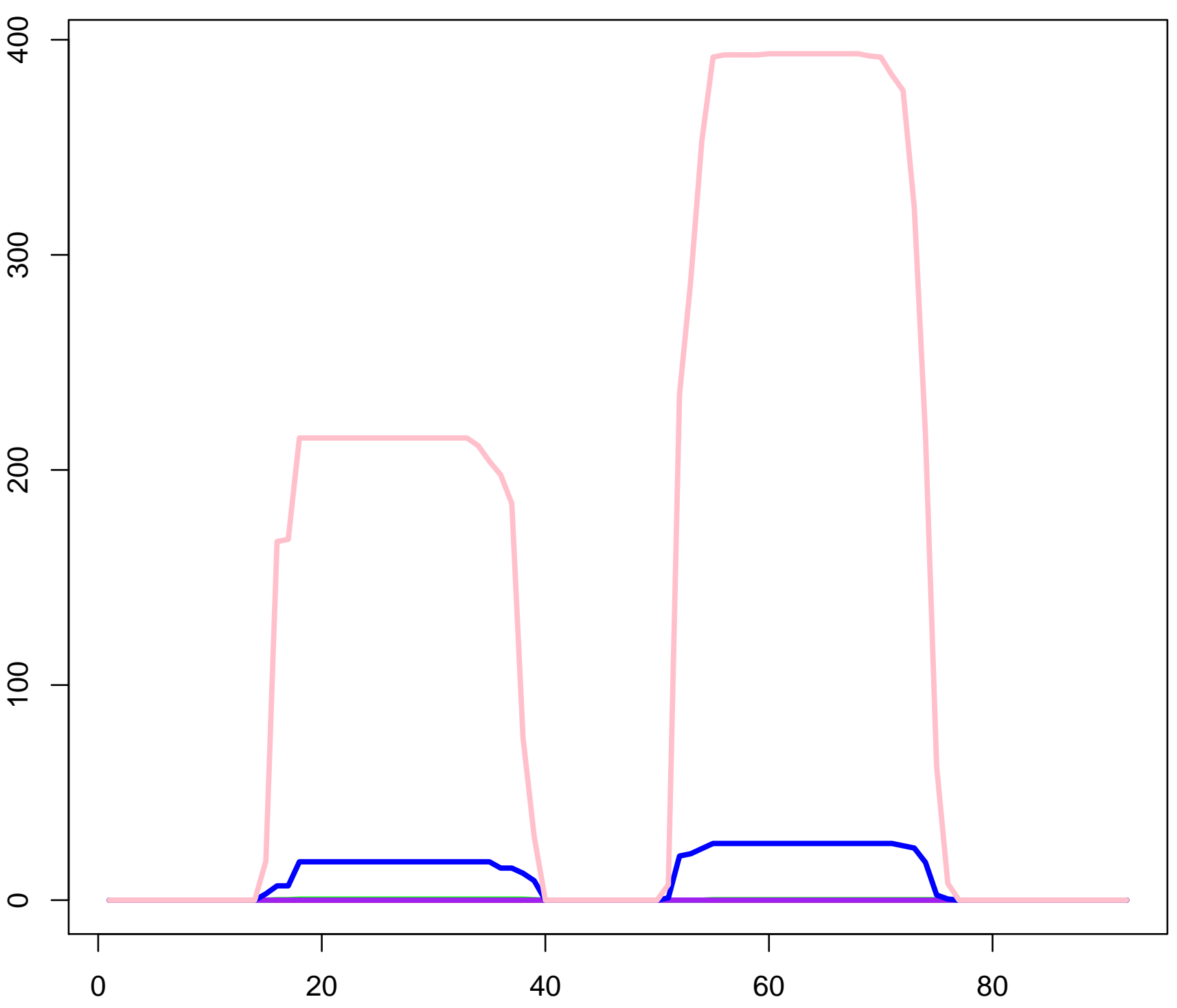

Egg $\longrightarrow$ St. 7-8 $\longrightarrow$ St. $12 \longrightarrow$ St 18-20 St. 34-36 St. 56 
Scaffold1376_1107396-1107479(-) mir-103

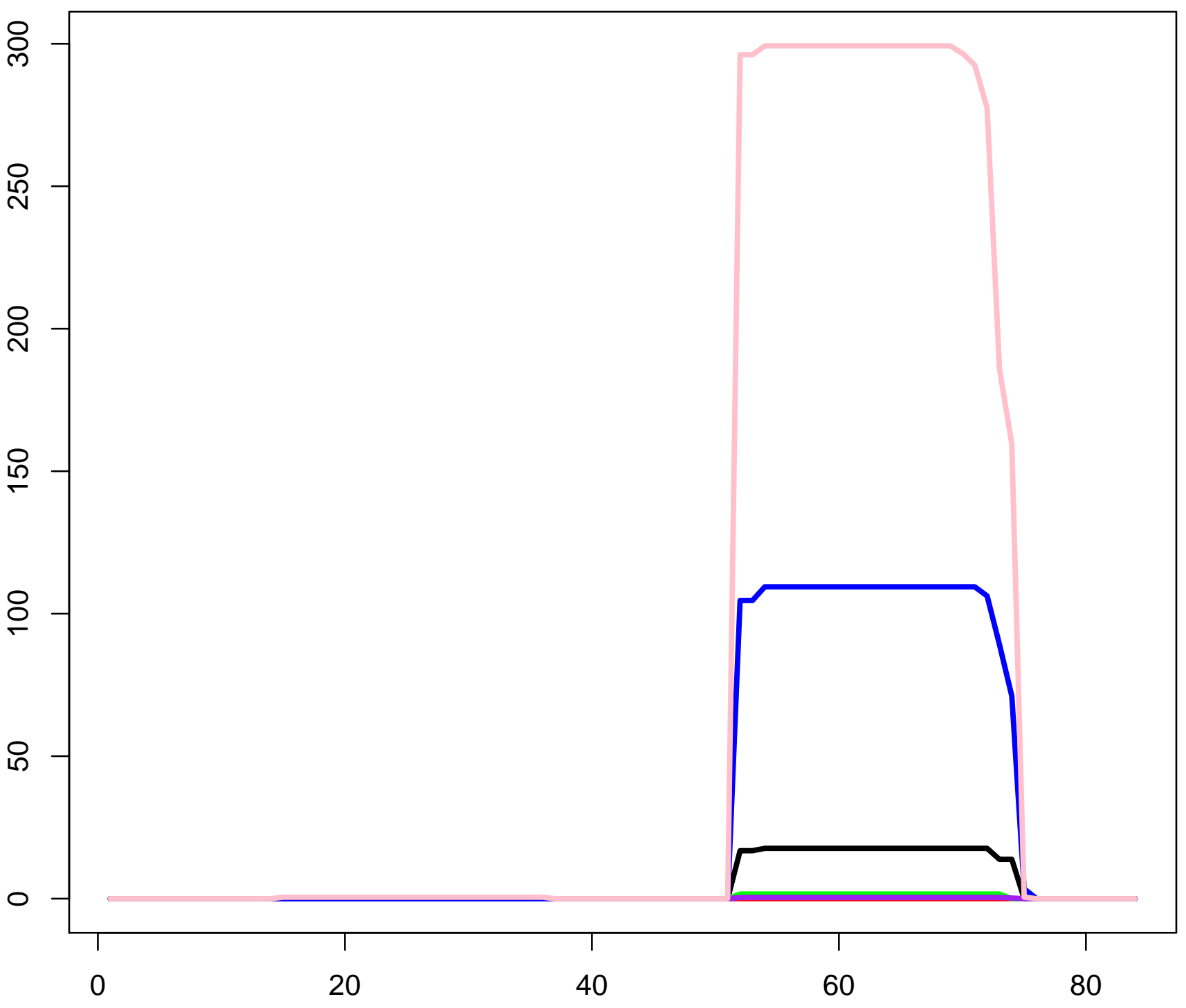


Scaffold13944_101763-101843(-) mir-181b-1

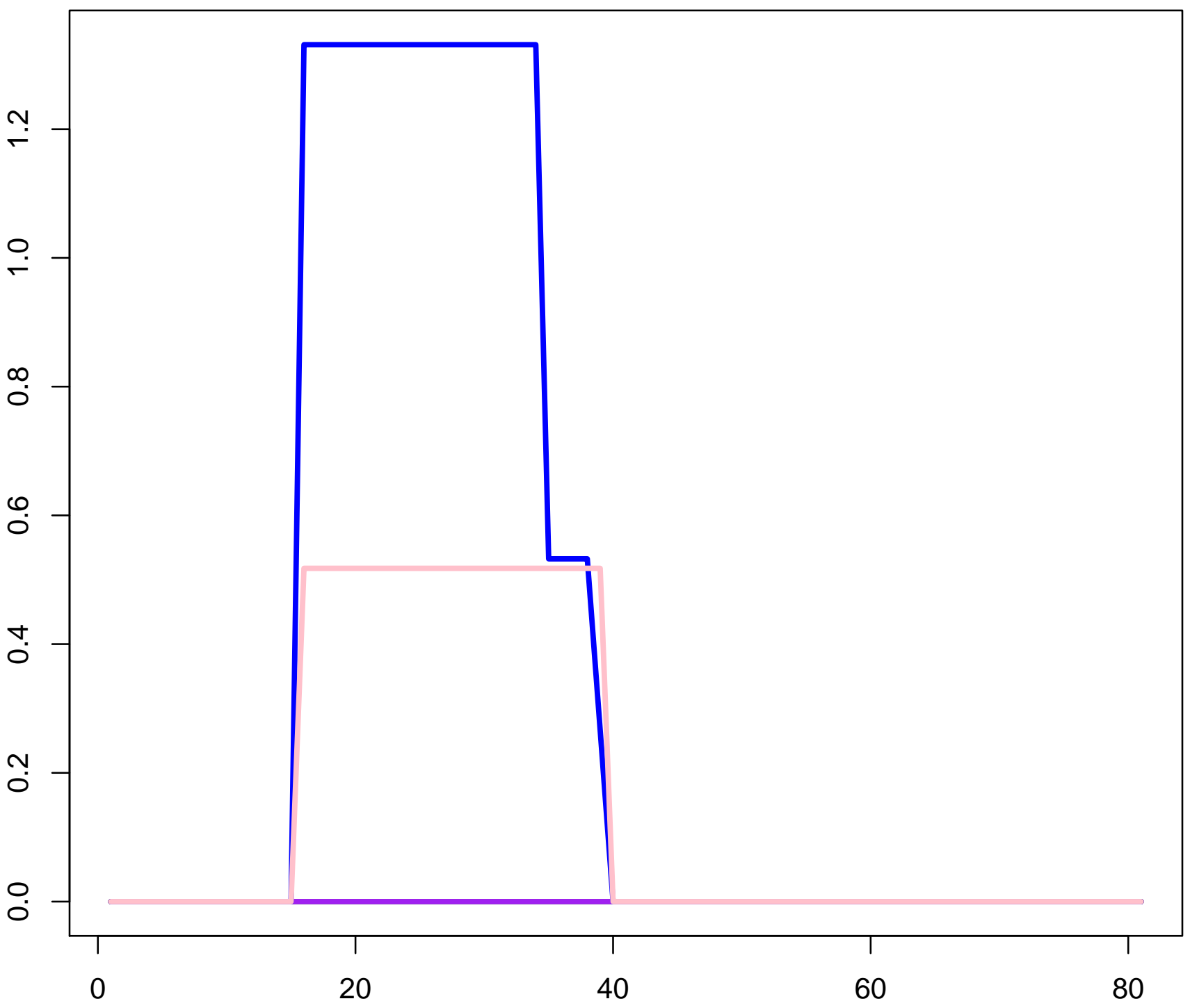

$\longrightarrow$ Egg $\longrightarrow$ St. 7-8 St. $12 \rightleftharpoons$ St 18-20 St. 34-36 St. 56 
Scaffold13944_103793-103887(-) mir-181a-1

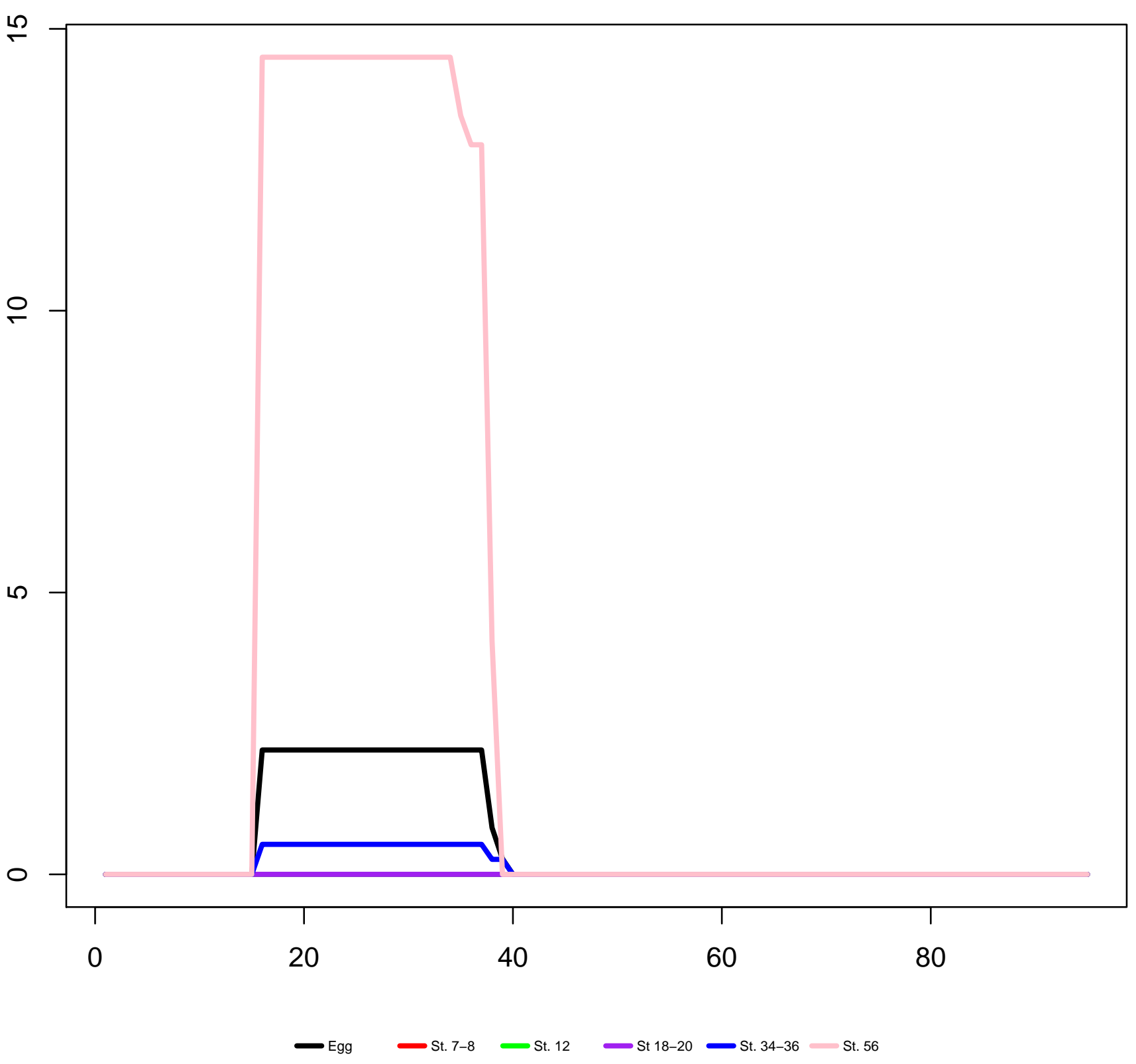


Scaffold141328_637933-638002(+) mir-206

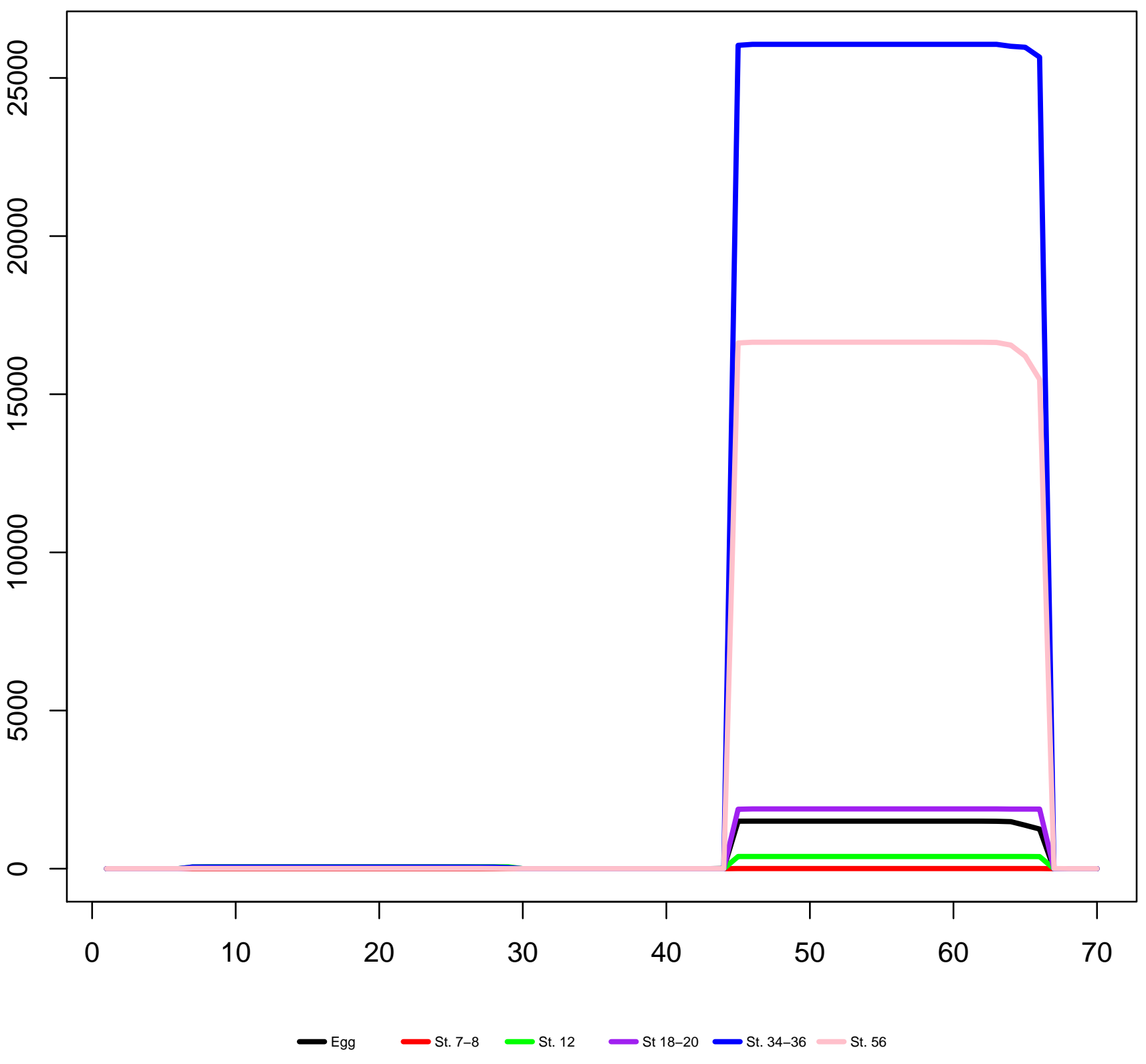


Scaffold141328_640751-640829(+) mir-133b

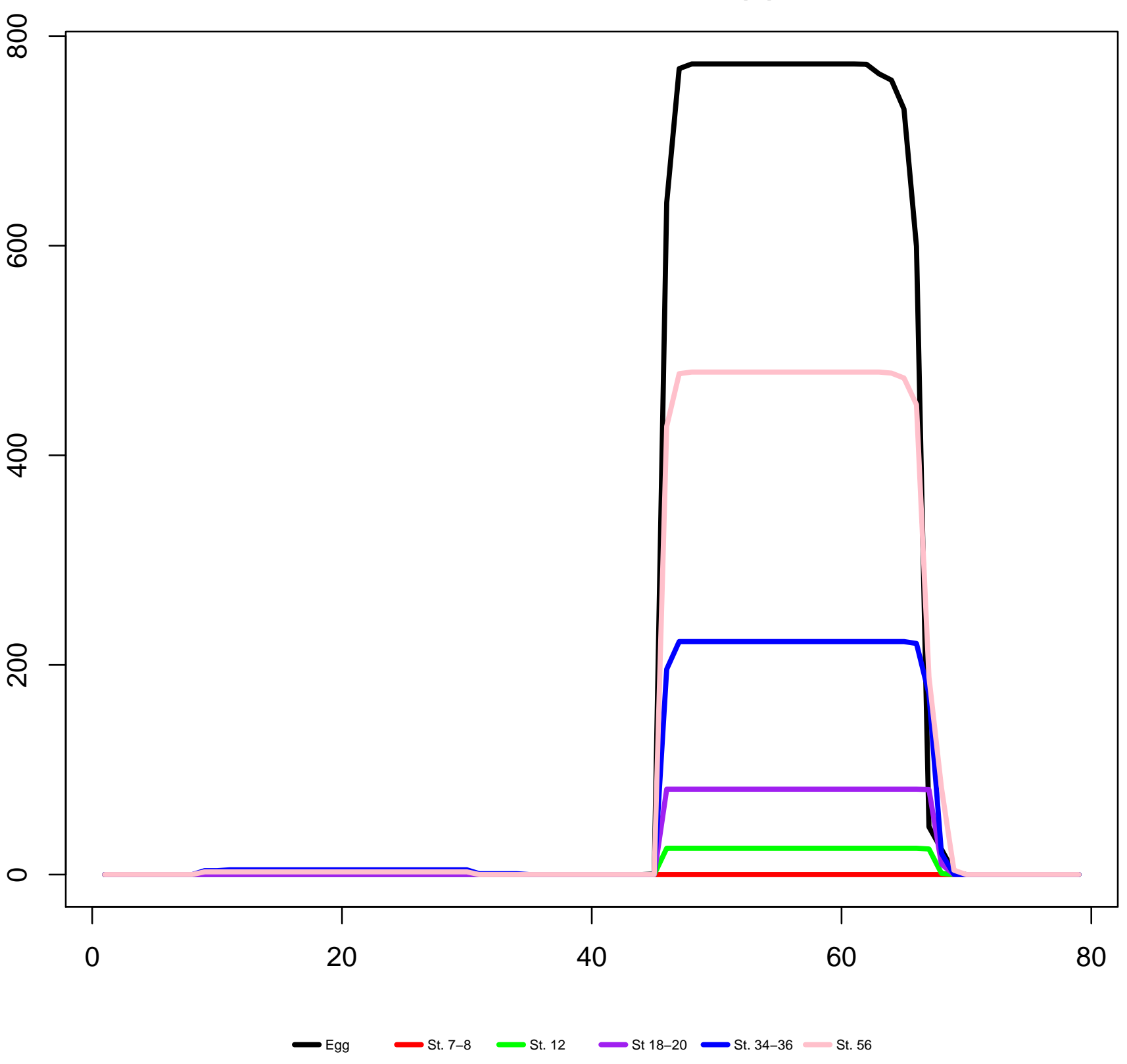


Scaffold147129_69377-69468(-) mir-219

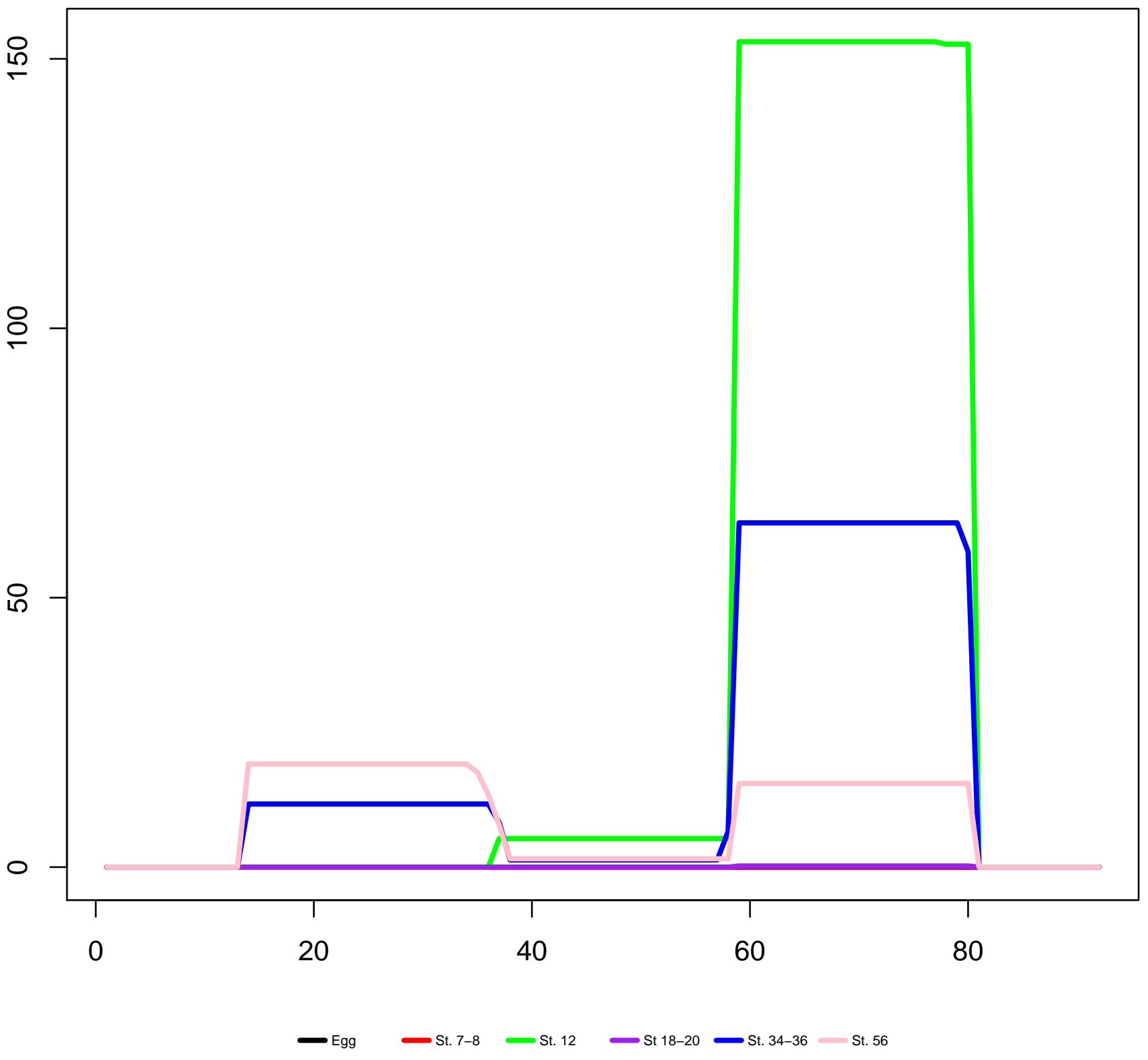


Scaffold14786_388703-388775(-) mir-1662

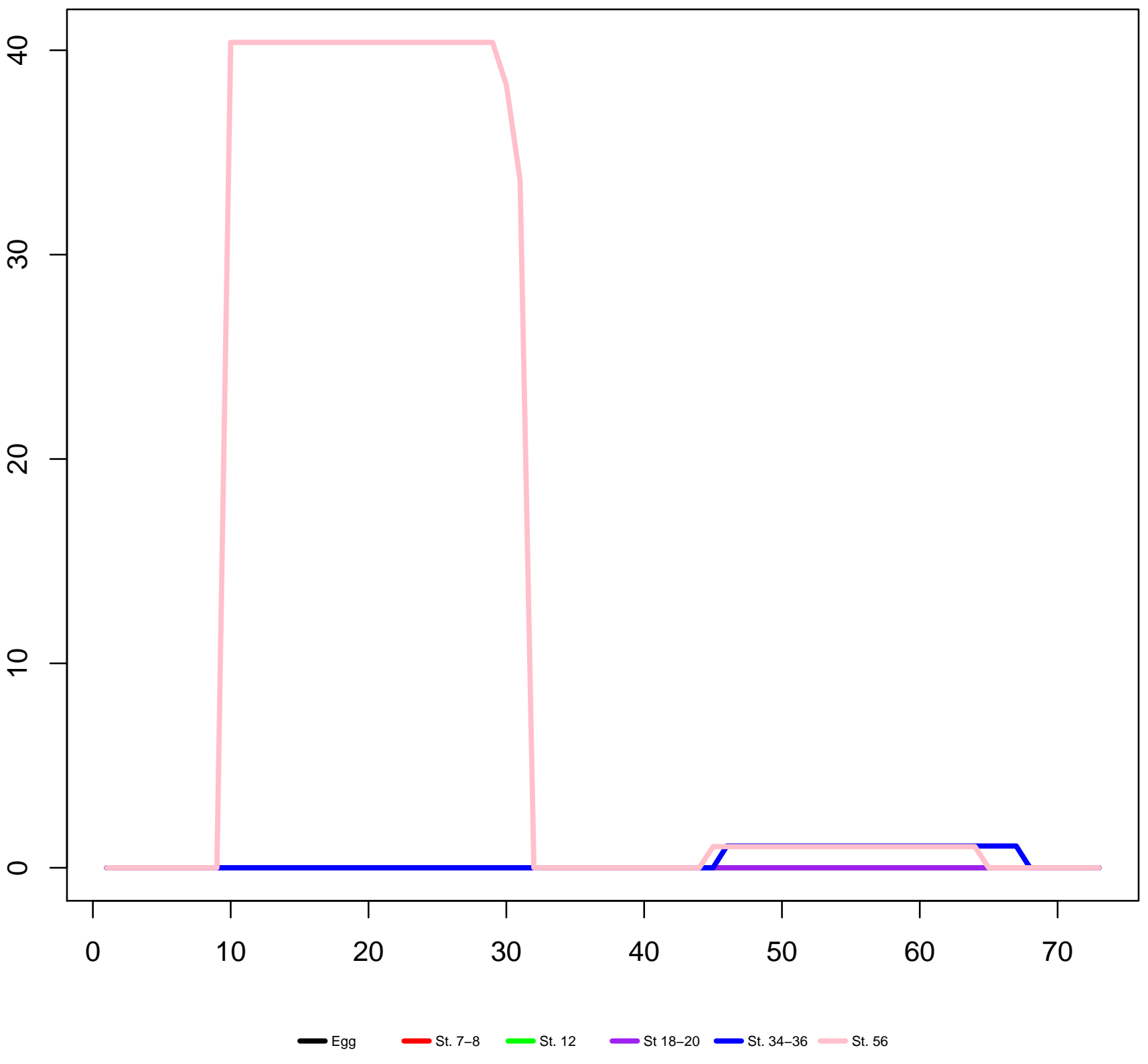


Scaffold15487_669554-669625(-) mir-10c

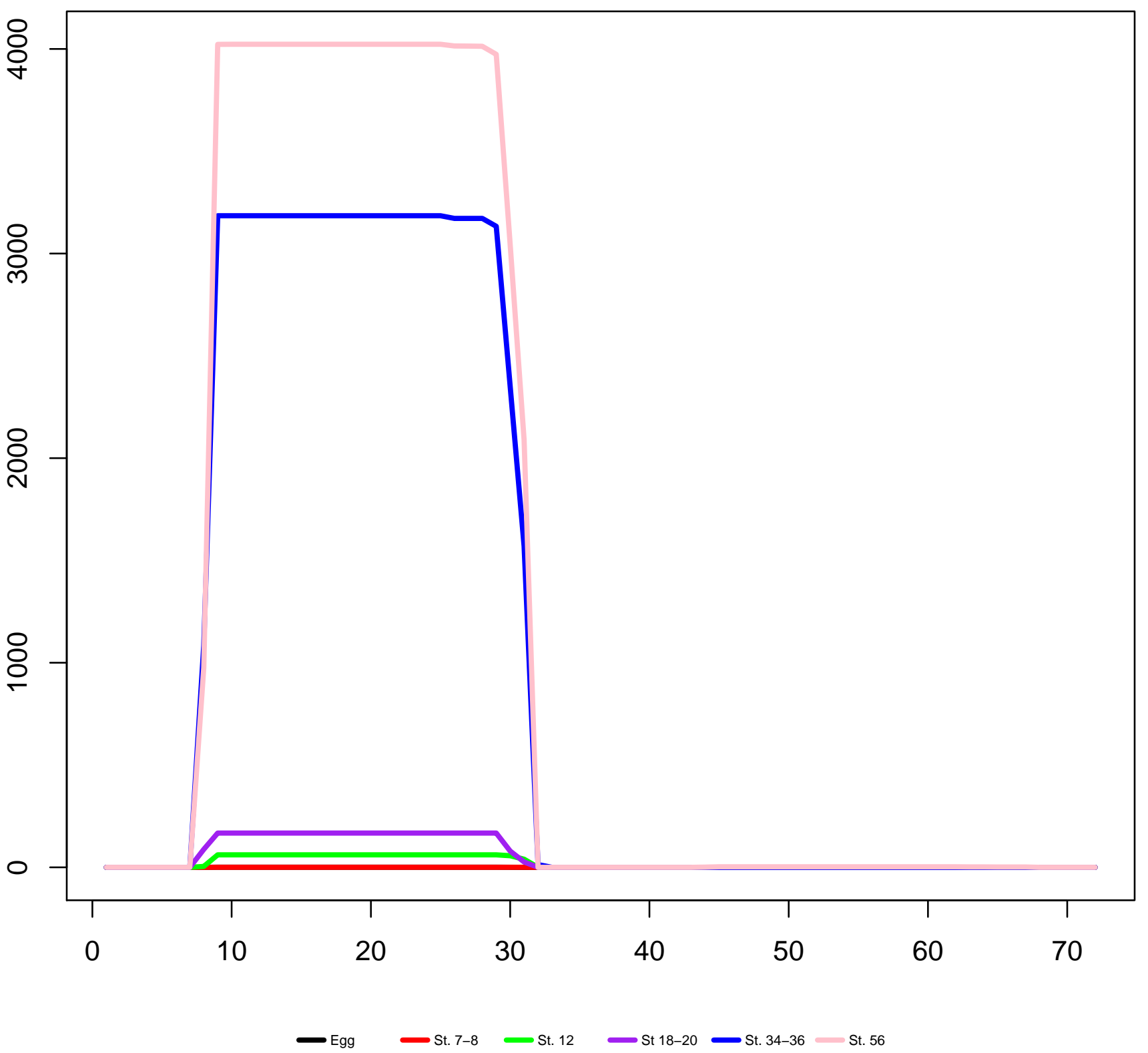




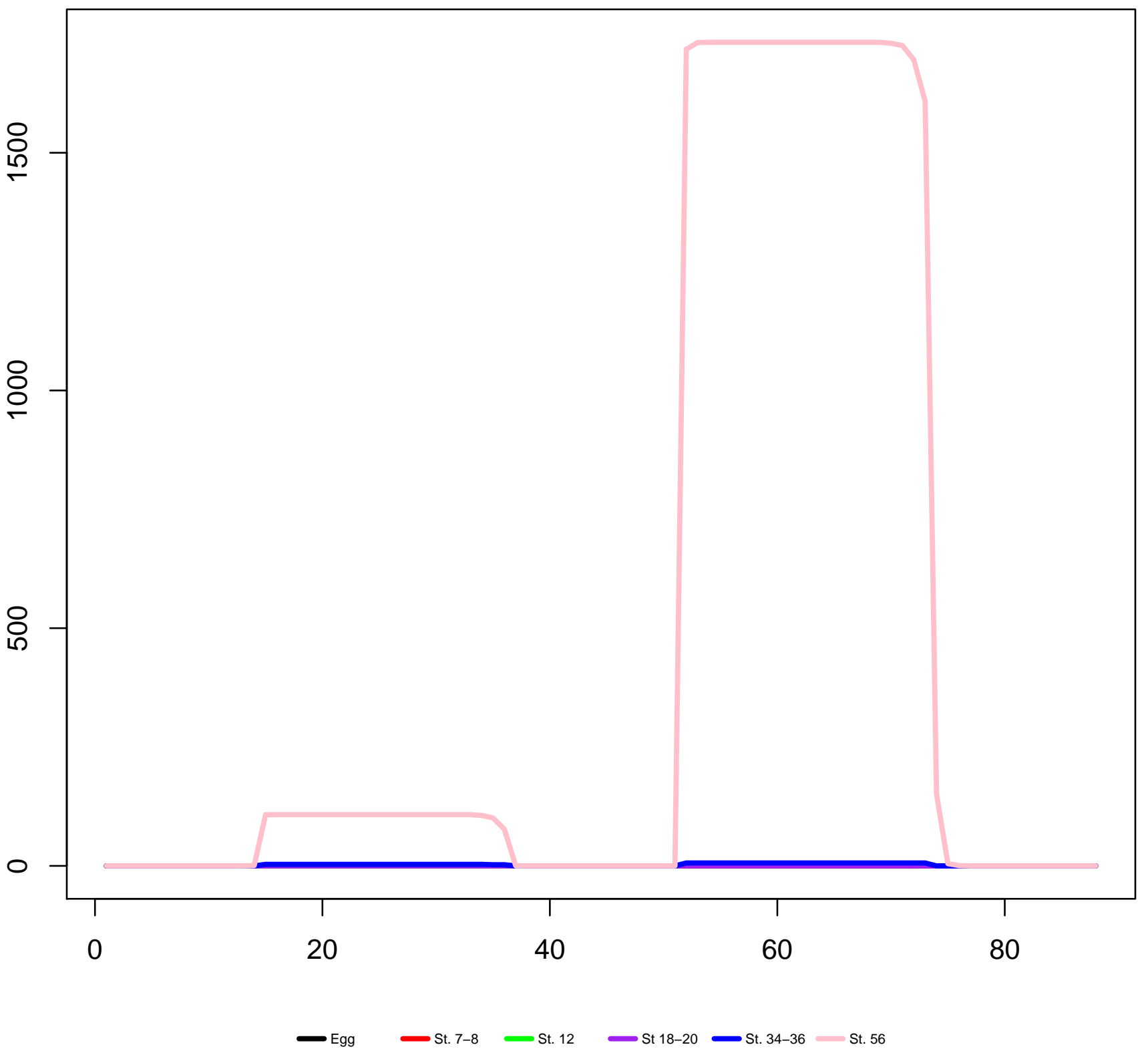




\section{Scaffold1583_763252-763334(-) mir-30c}

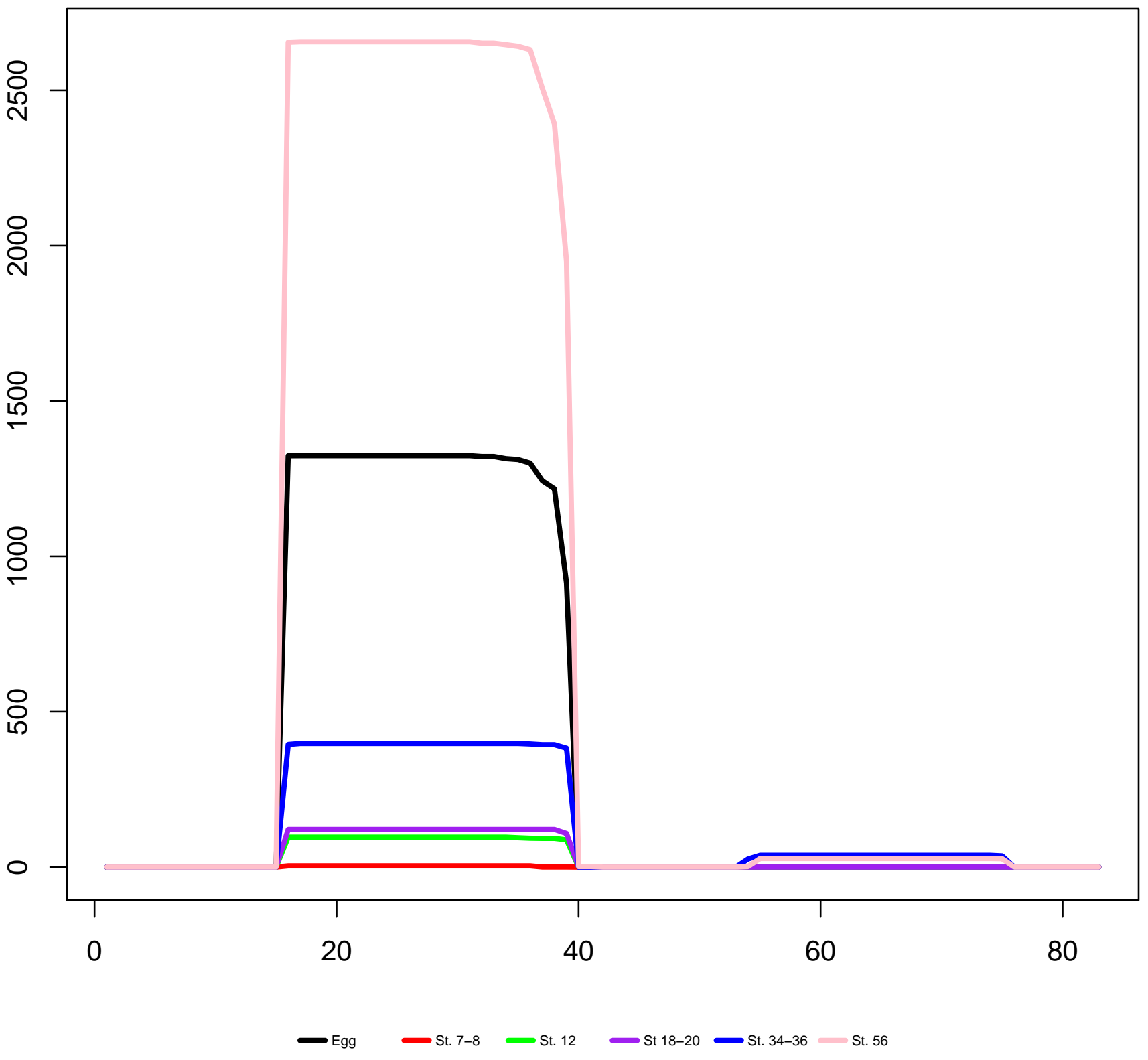


Scaffold1583_774877-774966(-) mir-30a

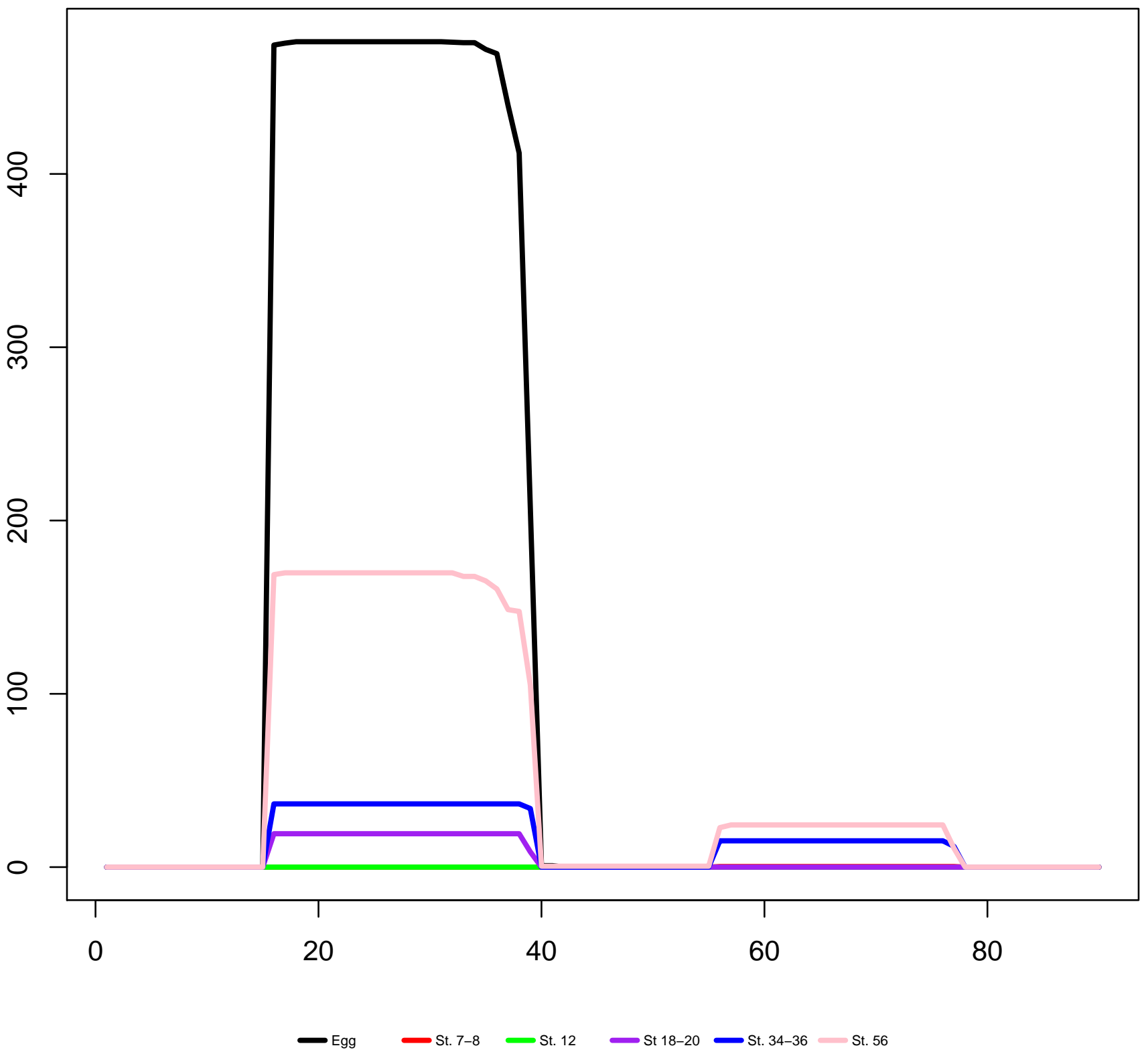


Scaffold160920_12-84(+) mir-92b

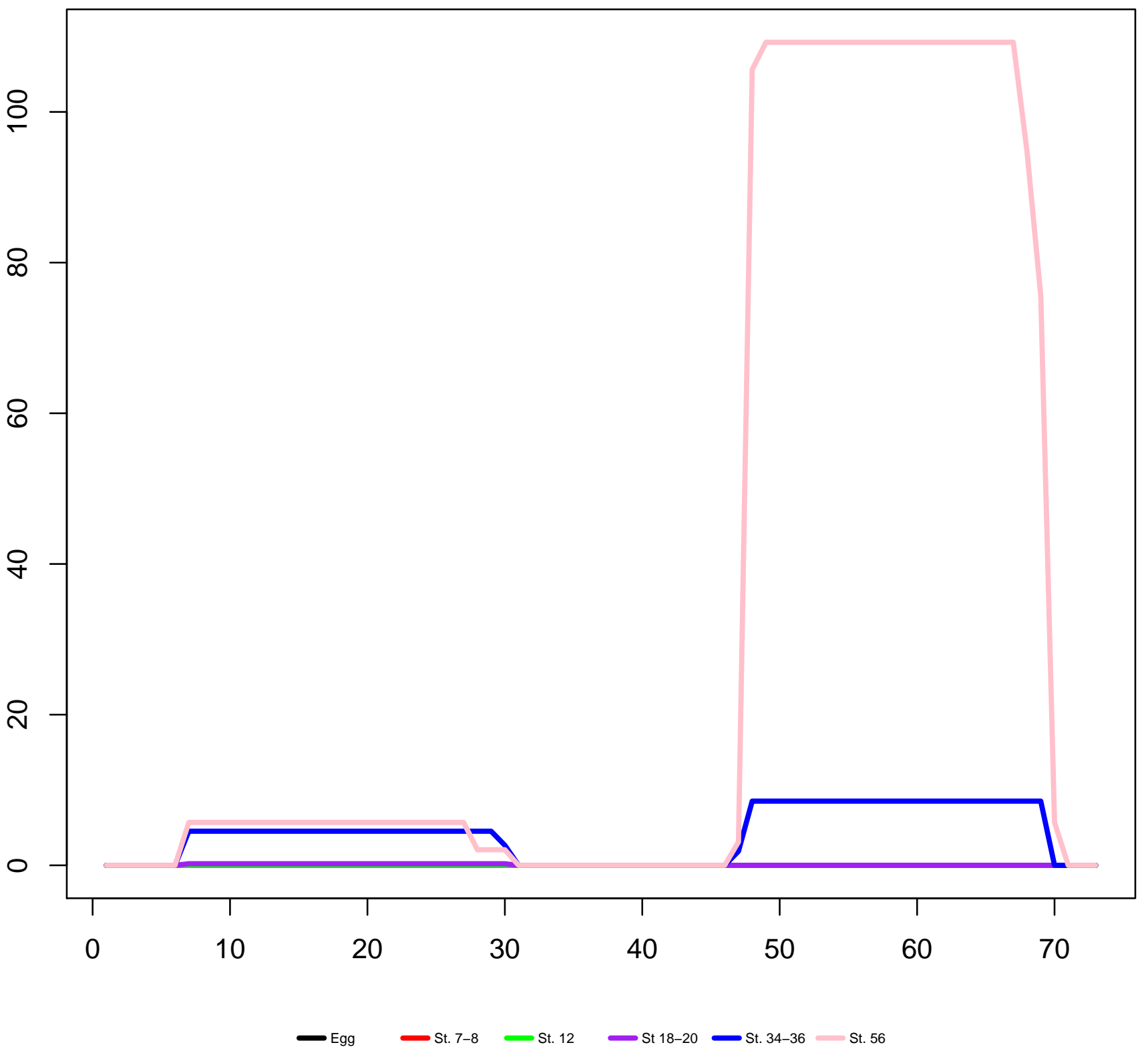


Scaffold16112_318821-318921(+) mir-383

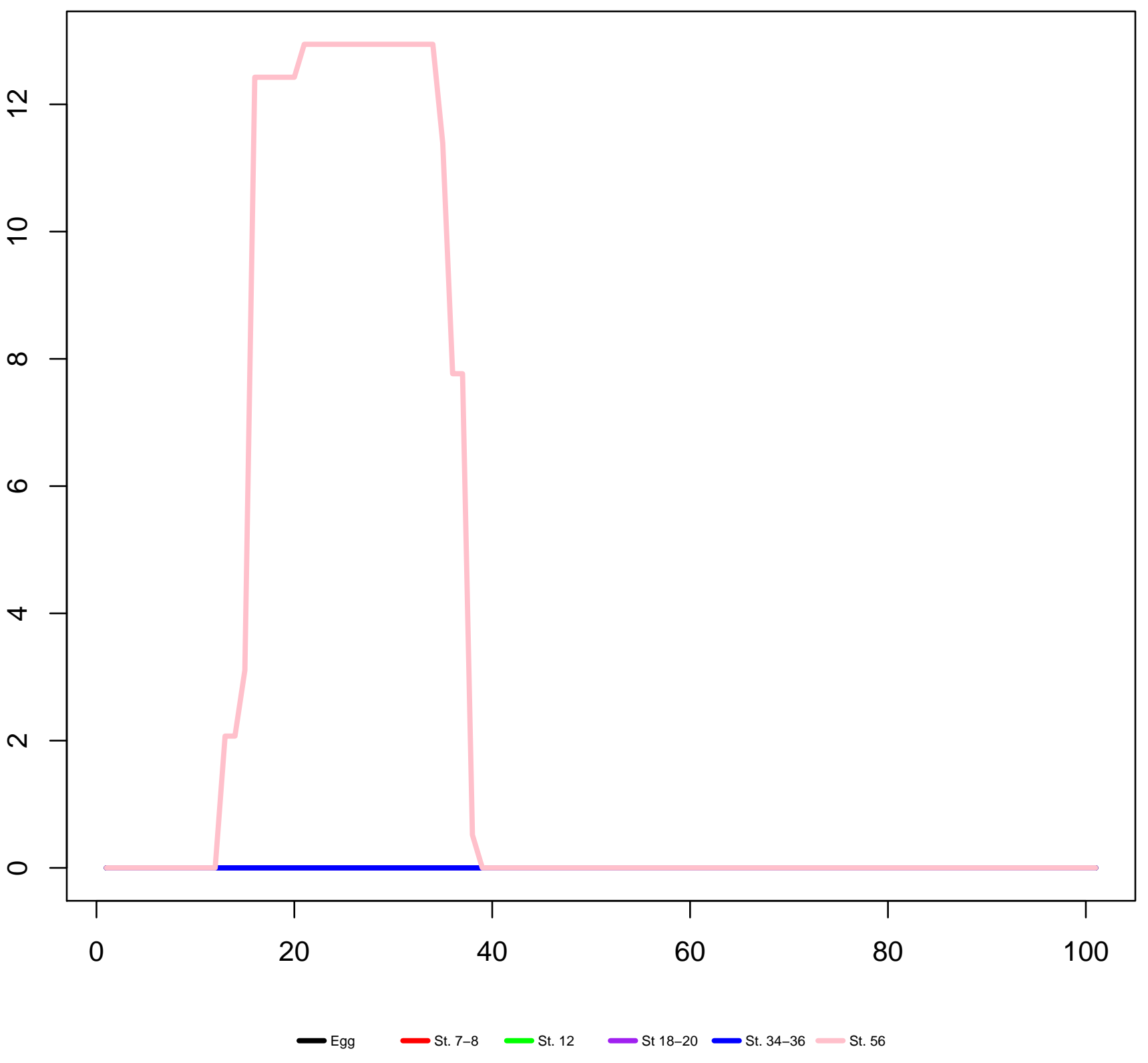


Scaffold164200_145969-146063(-) mir-140

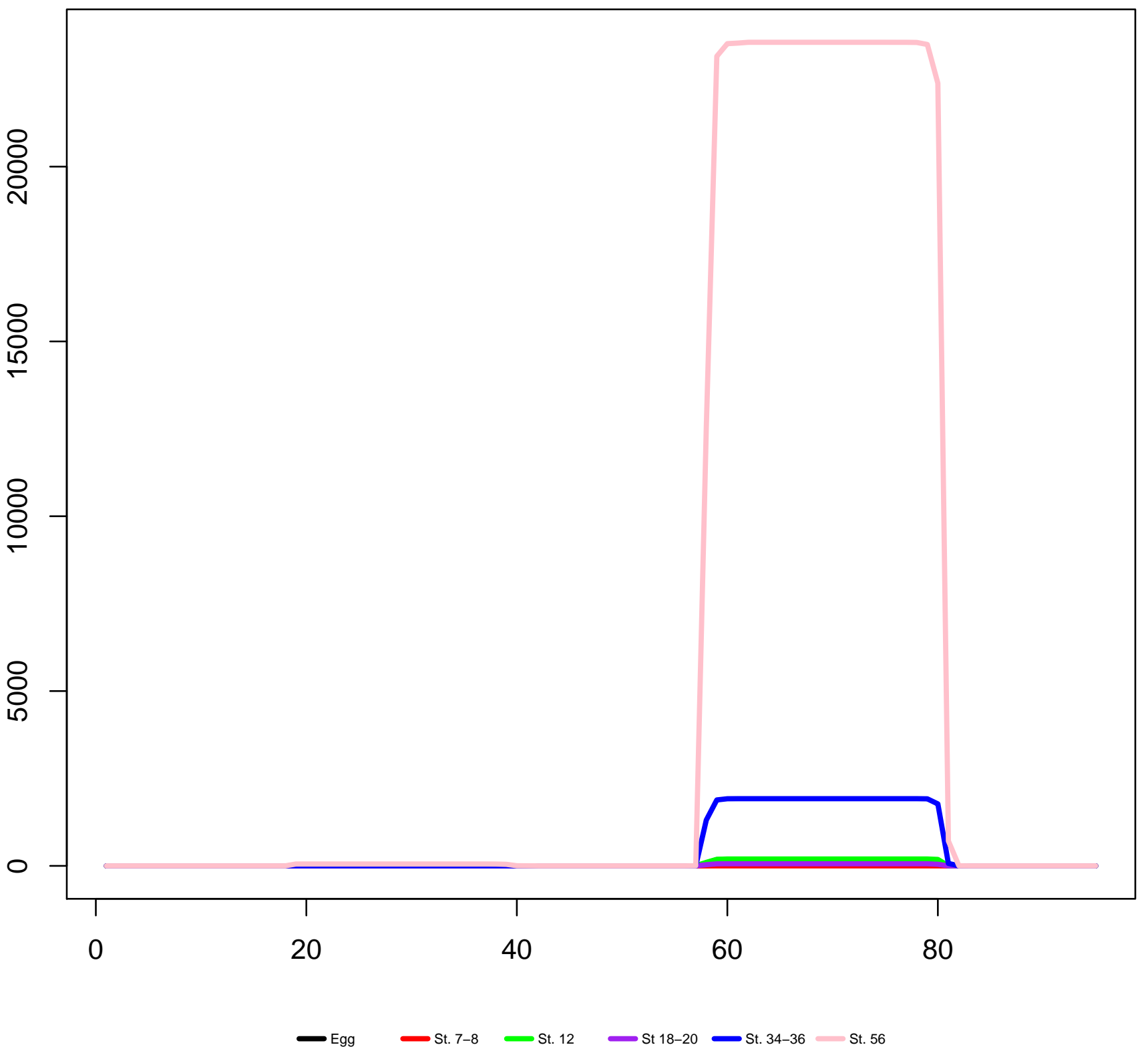


Scaffold1657_253815-253896(-) mir-460

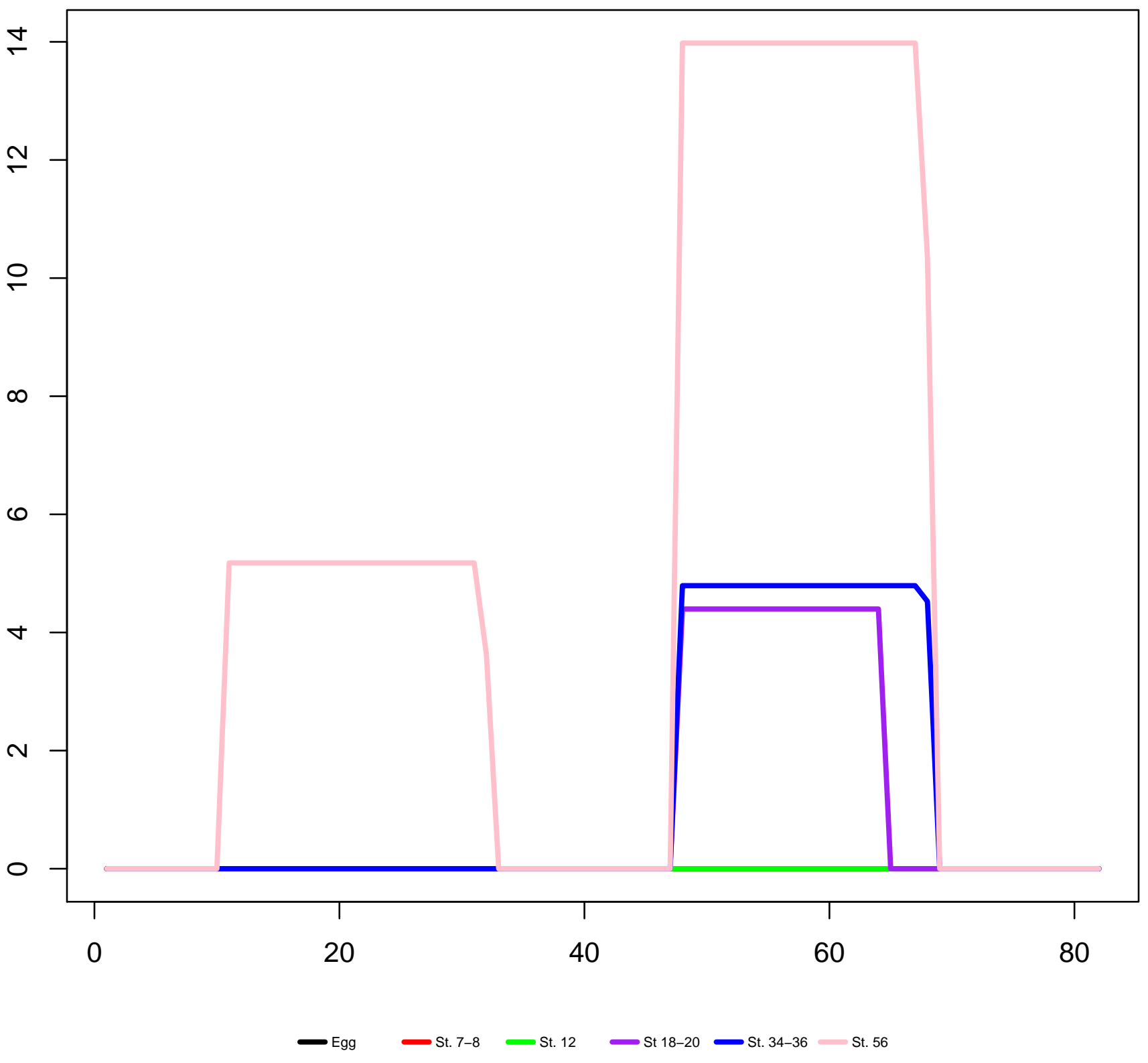


Scaffold16771_1013357-1013436(+) mir-184

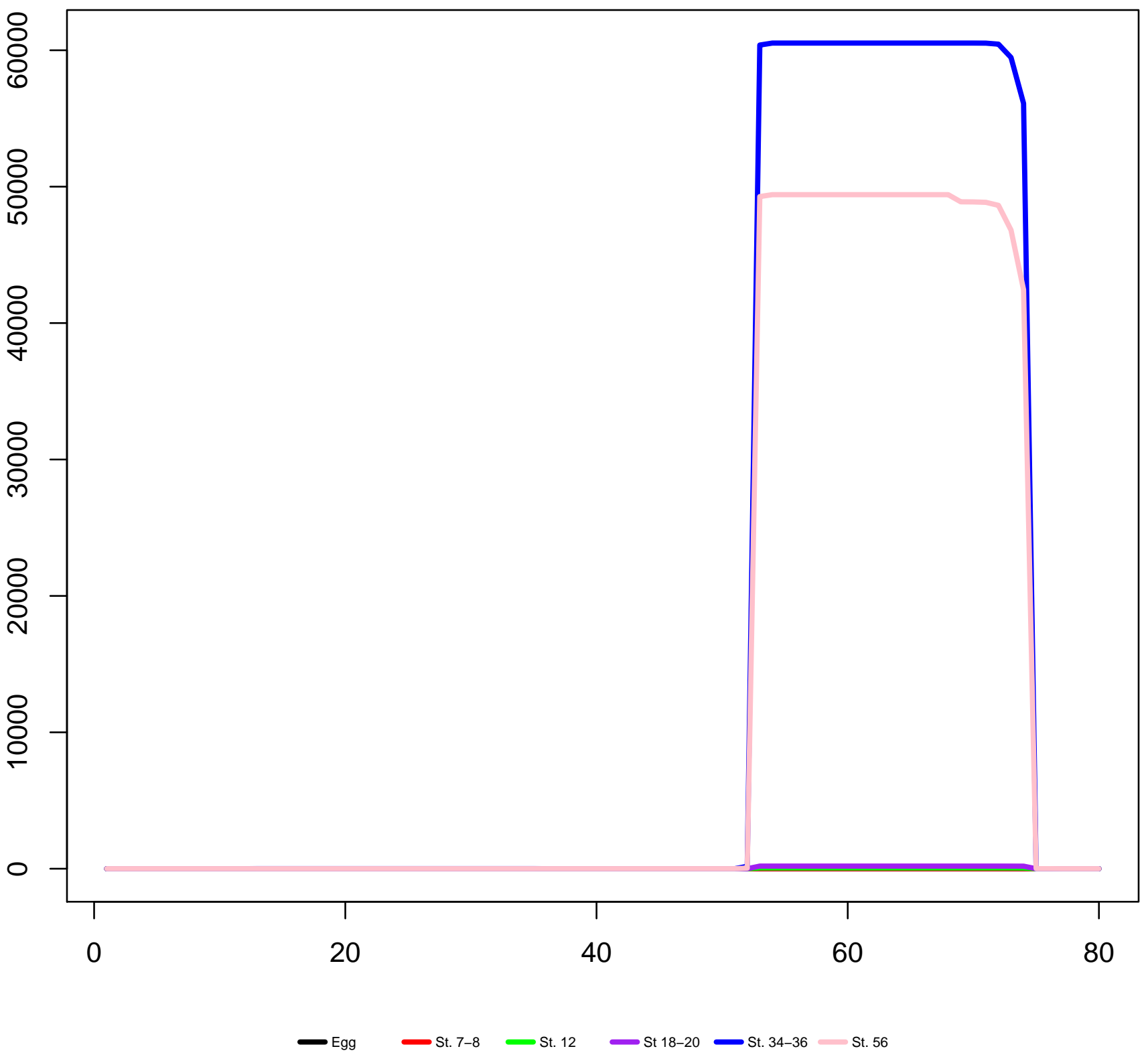


Scaffold168775_245472-245562(-) mir-133a-1

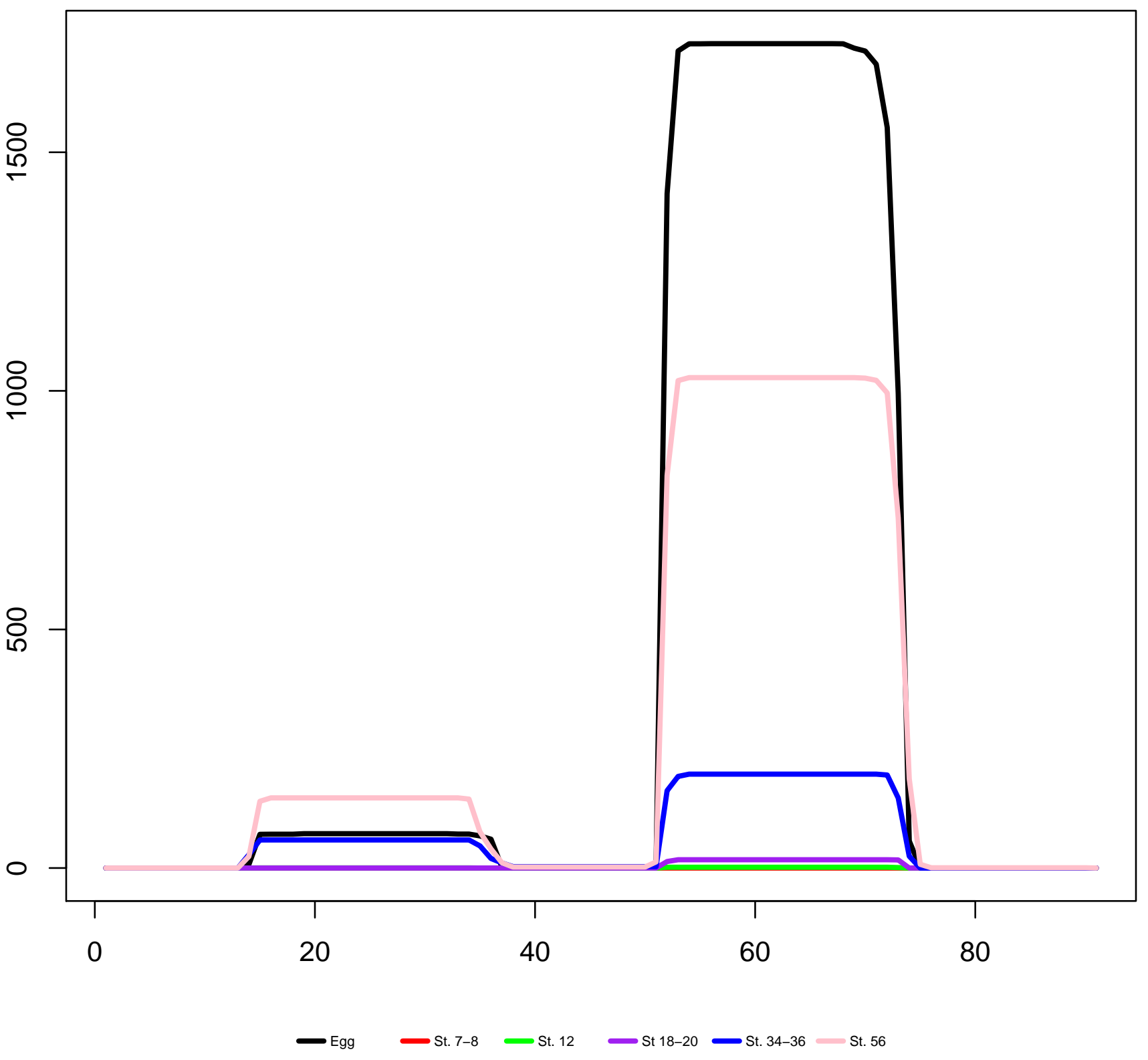


Scaffold168775_261843-261919(-) mir-1a-2

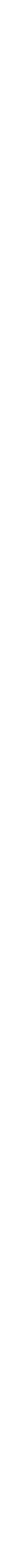


Scaffold17216_646100-646188(-) mir-124a-1

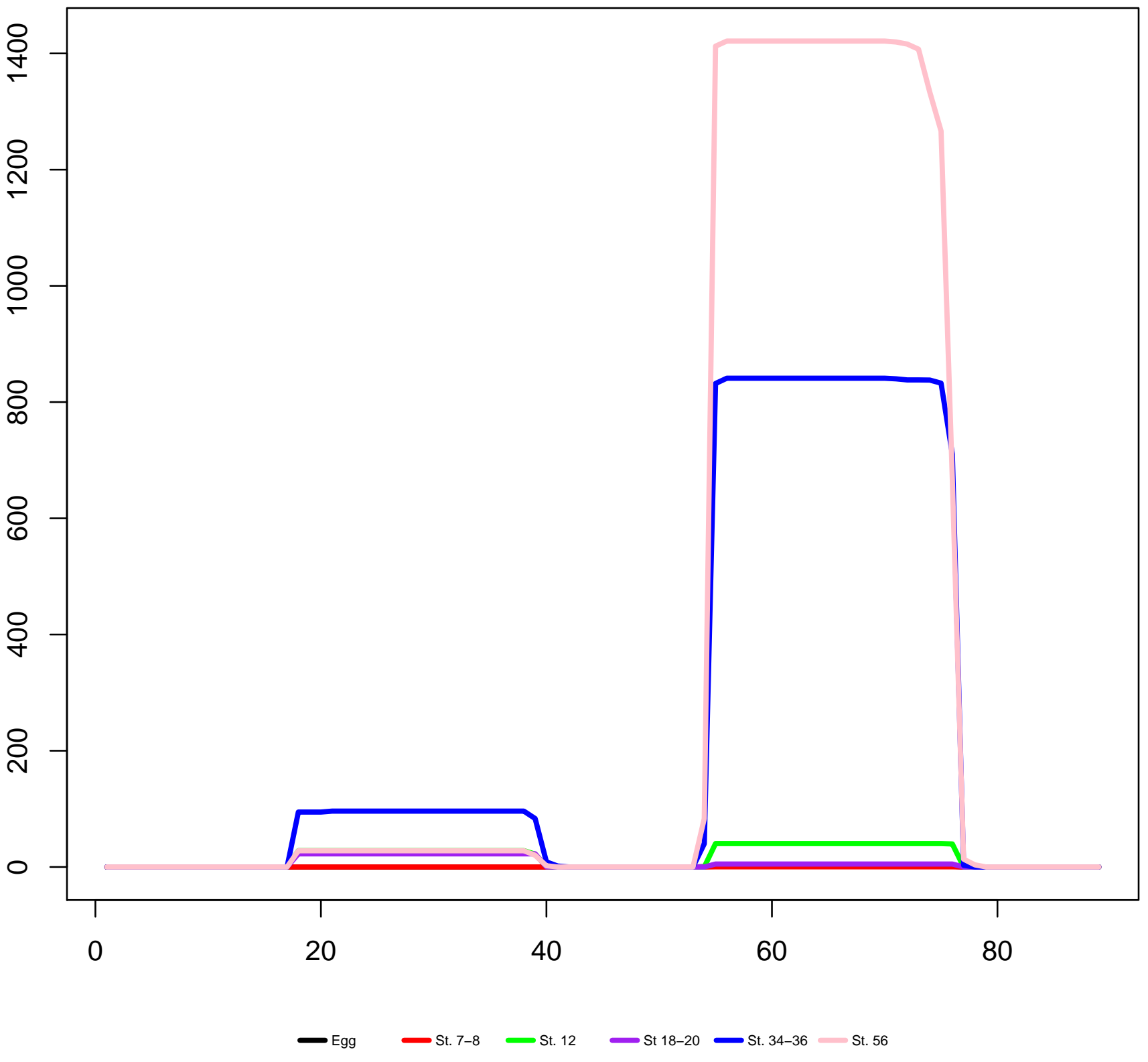


Scaffold172192_1-126(+) mir-135-2

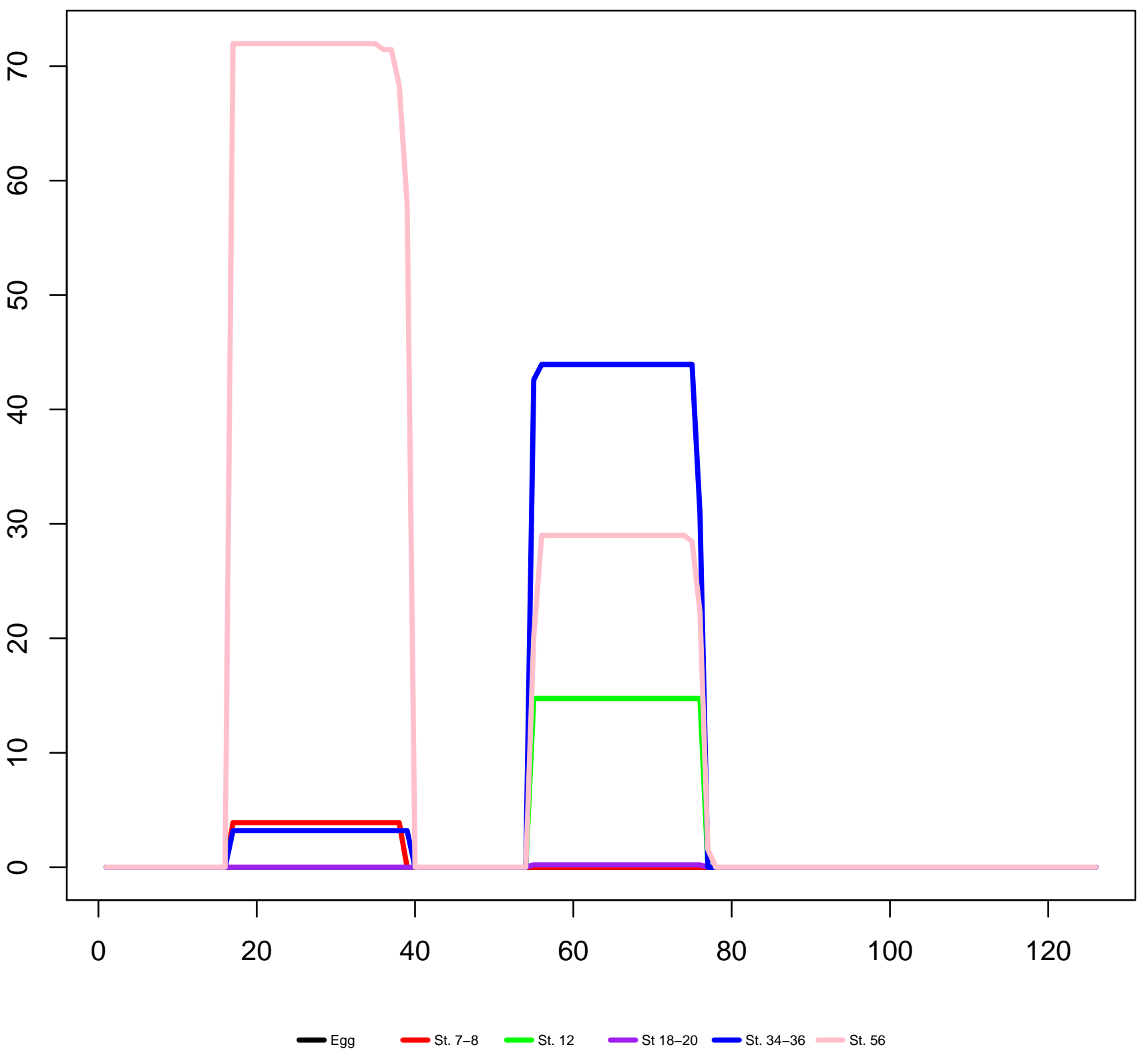


Scaffold17362_56334-56397(-) mir-150

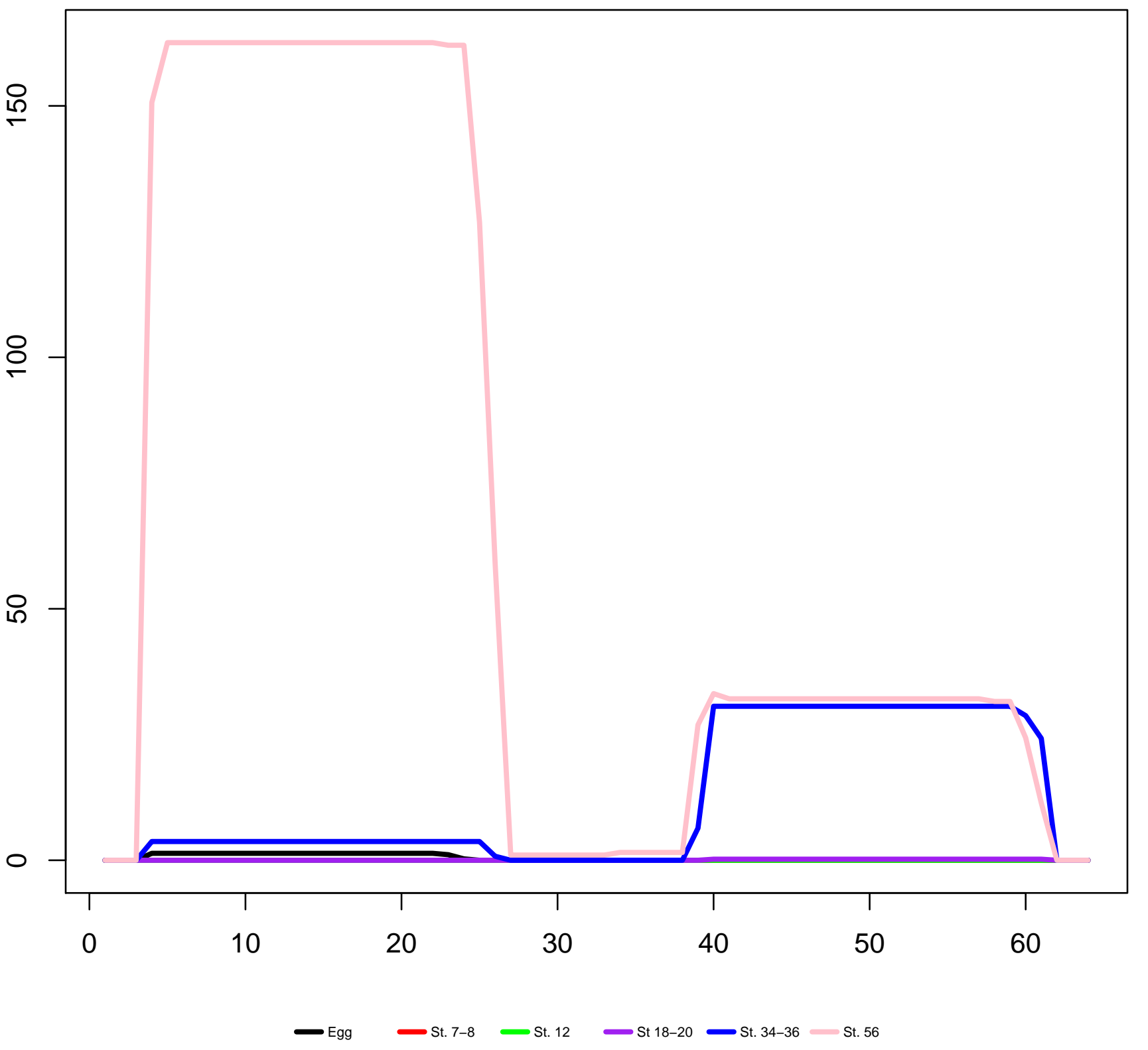


Scaffold17423_1047890-1047961(-) mir-205b

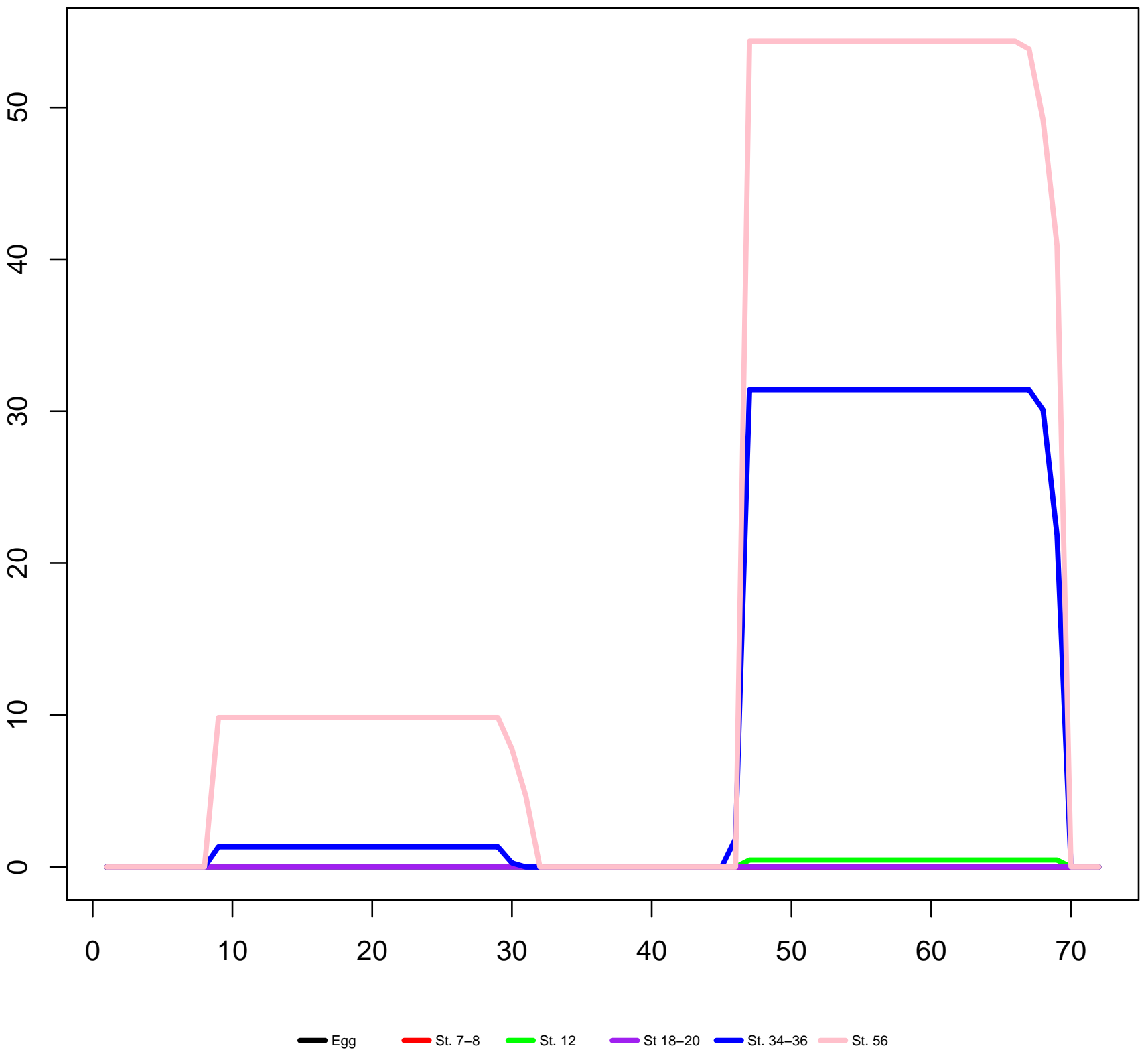


Scaffold175049_36239-36306(+) mir-27a

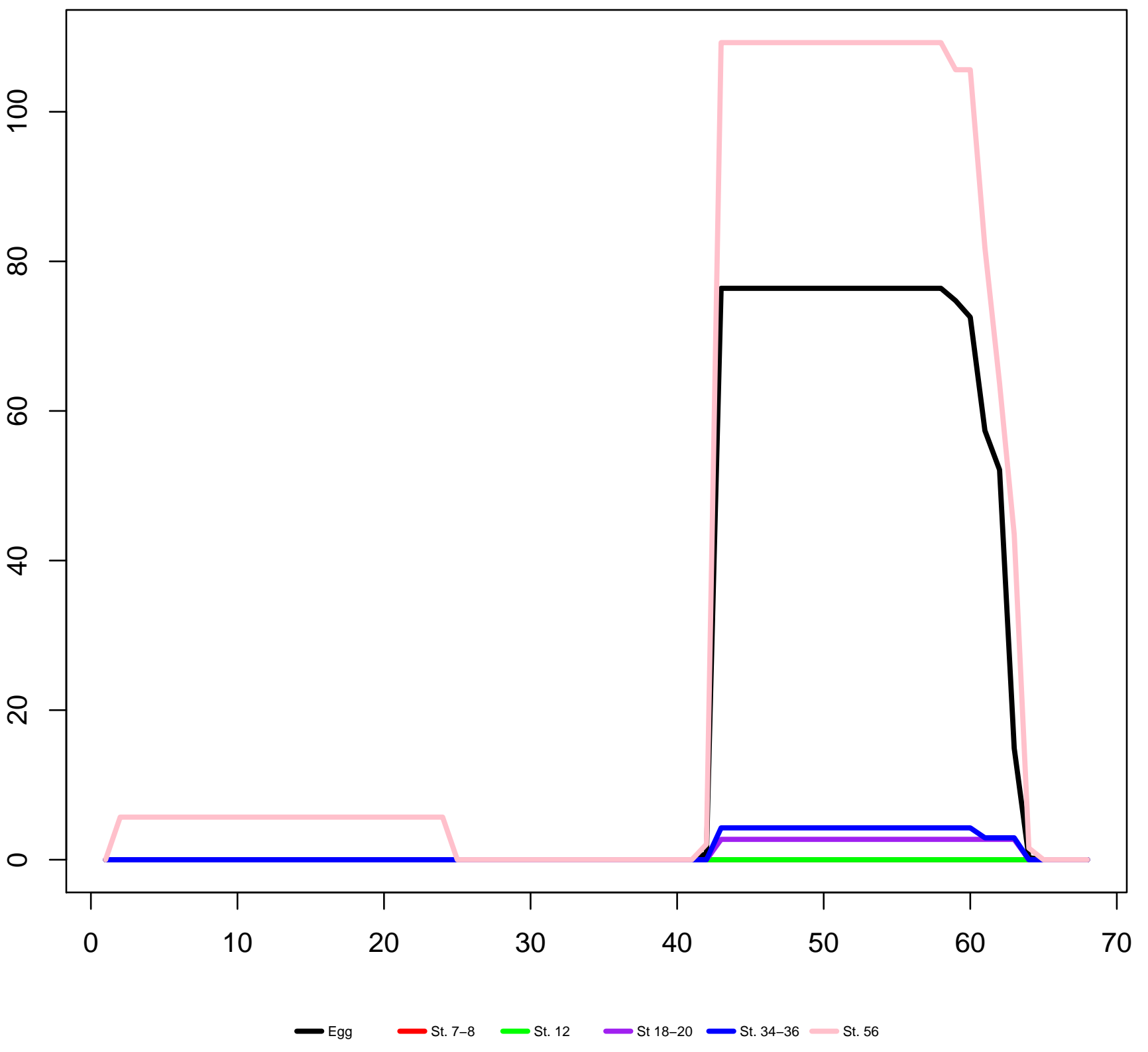


Scaffold176952_51630-51720(+) mir-210

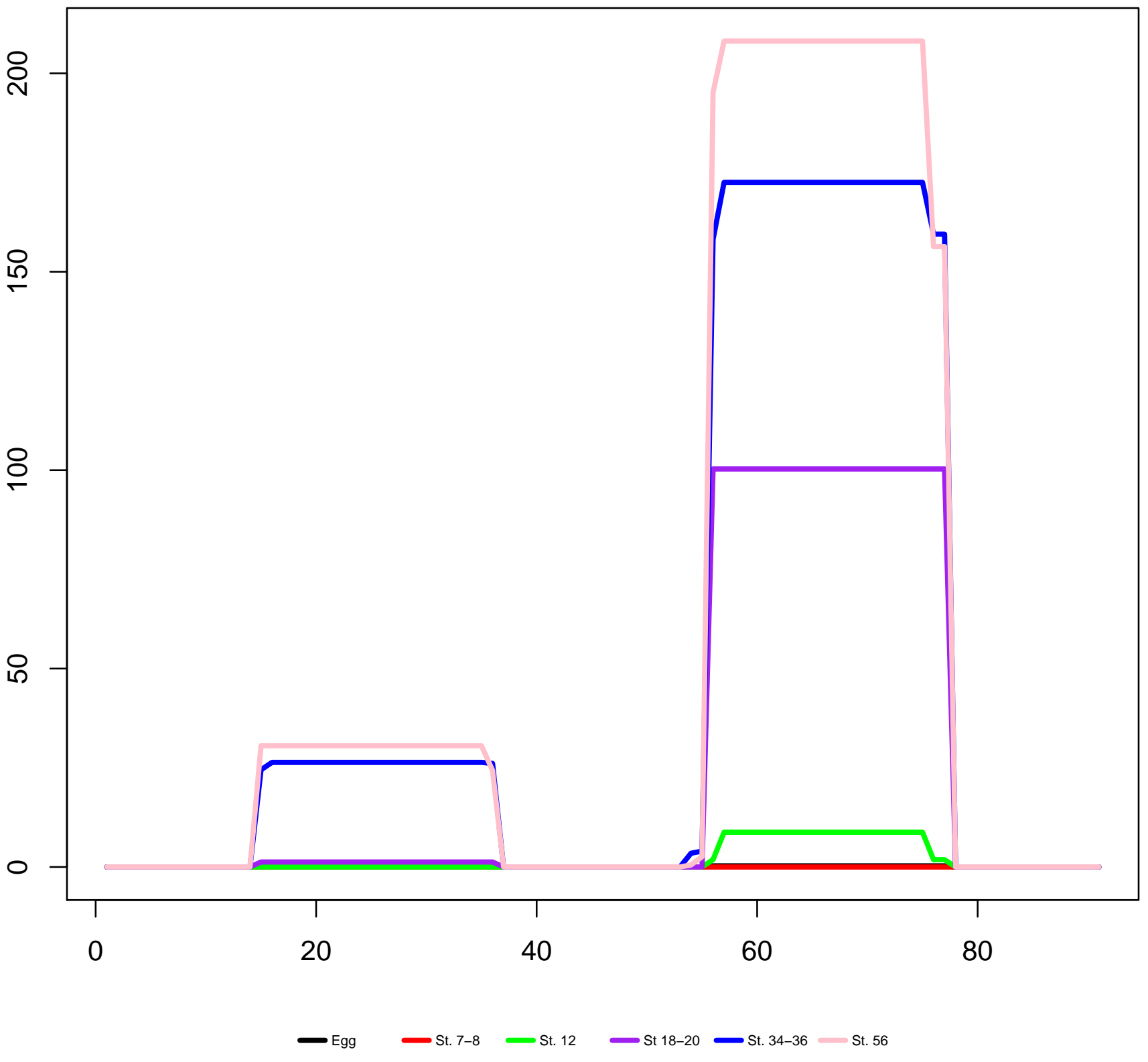


Scaffold18050_1210919-1211042(-) mir-214

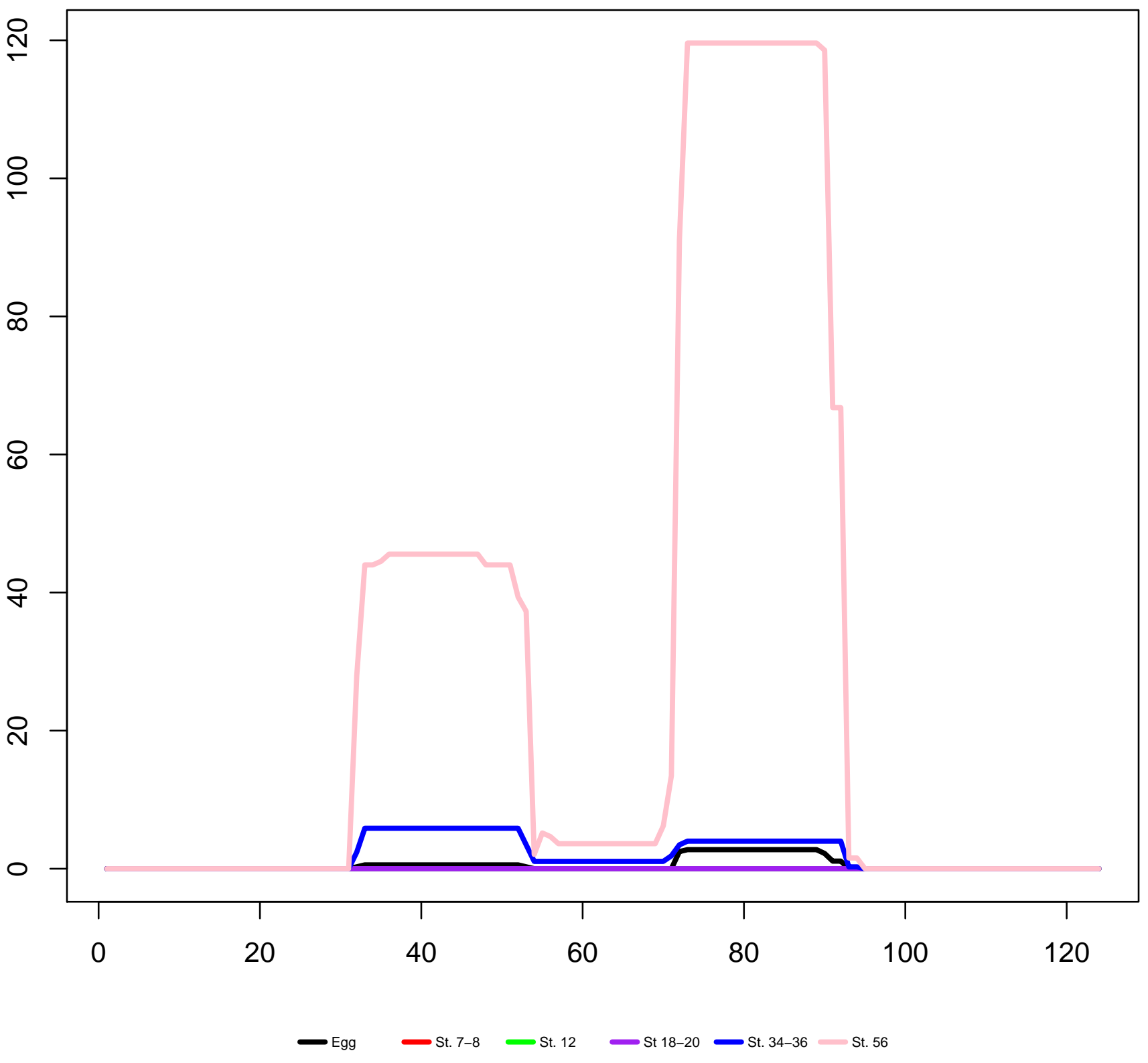


Scaffold18050_1217760-1217860(-) mir-199a-1

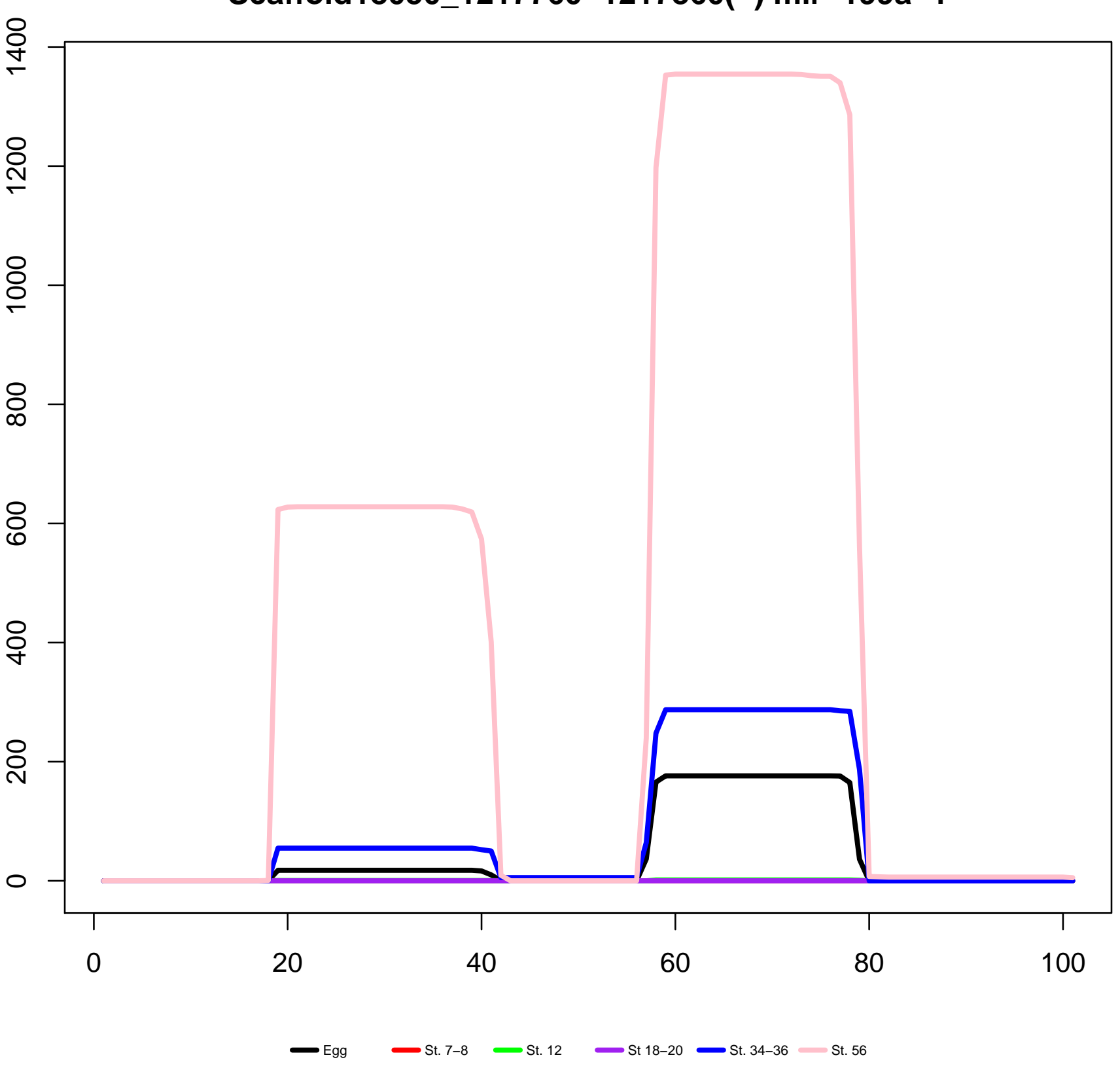


Scaffold18193_1347827-1347906(+) mir-194

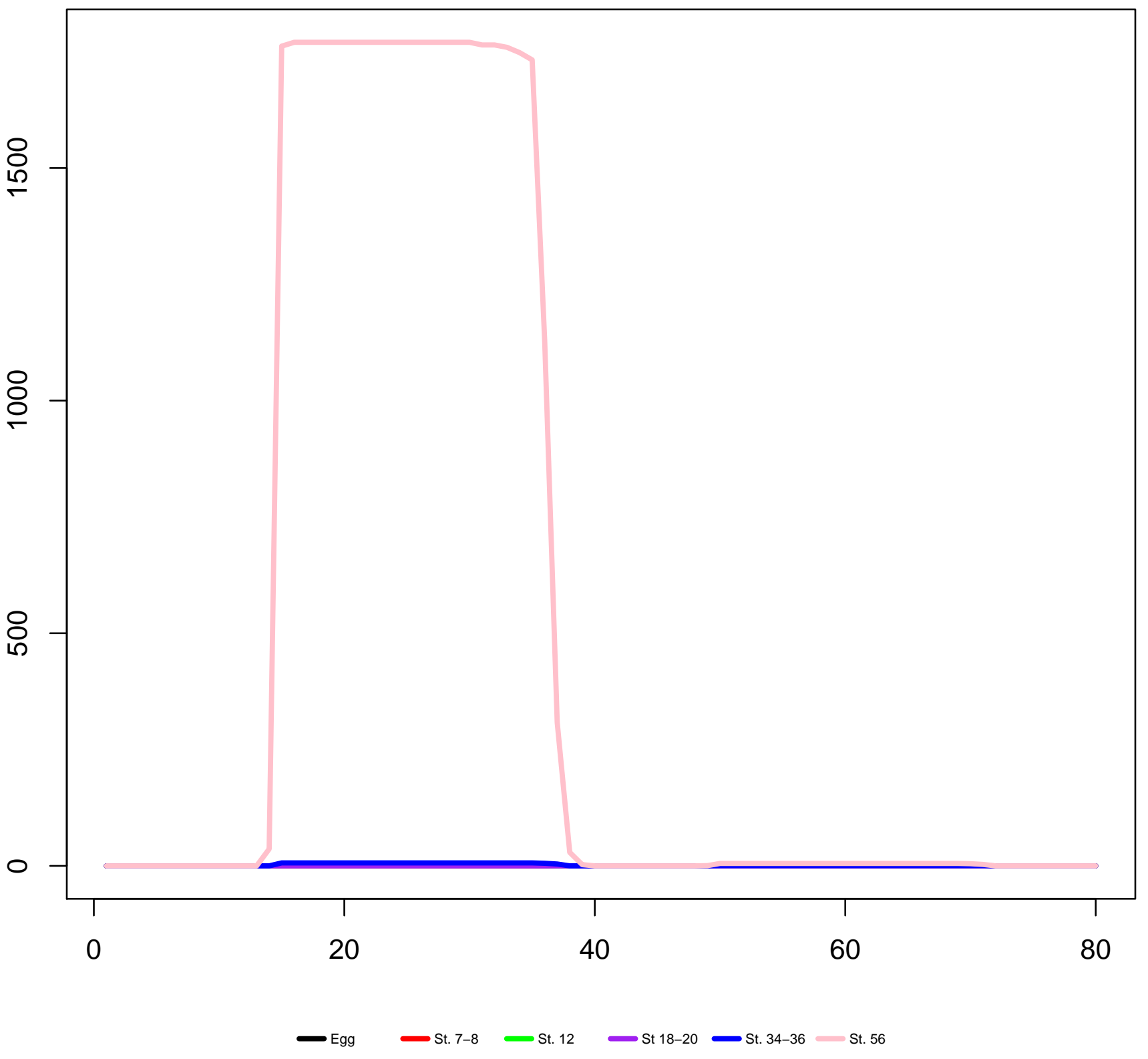


Scaffold18779_45658-45751(+) mir-34a

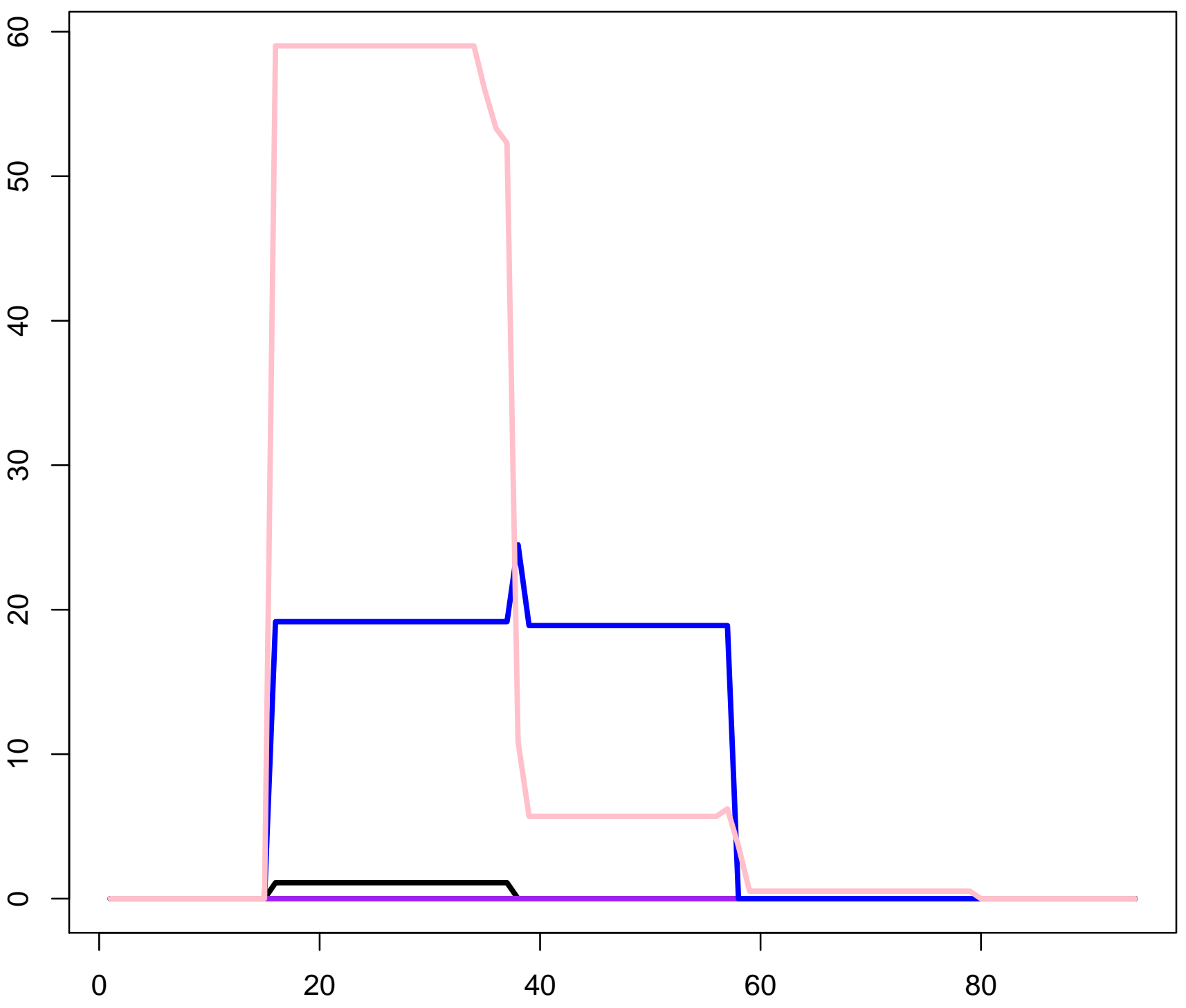


Scaffold18923_1099028-1099105(-) mir-204a

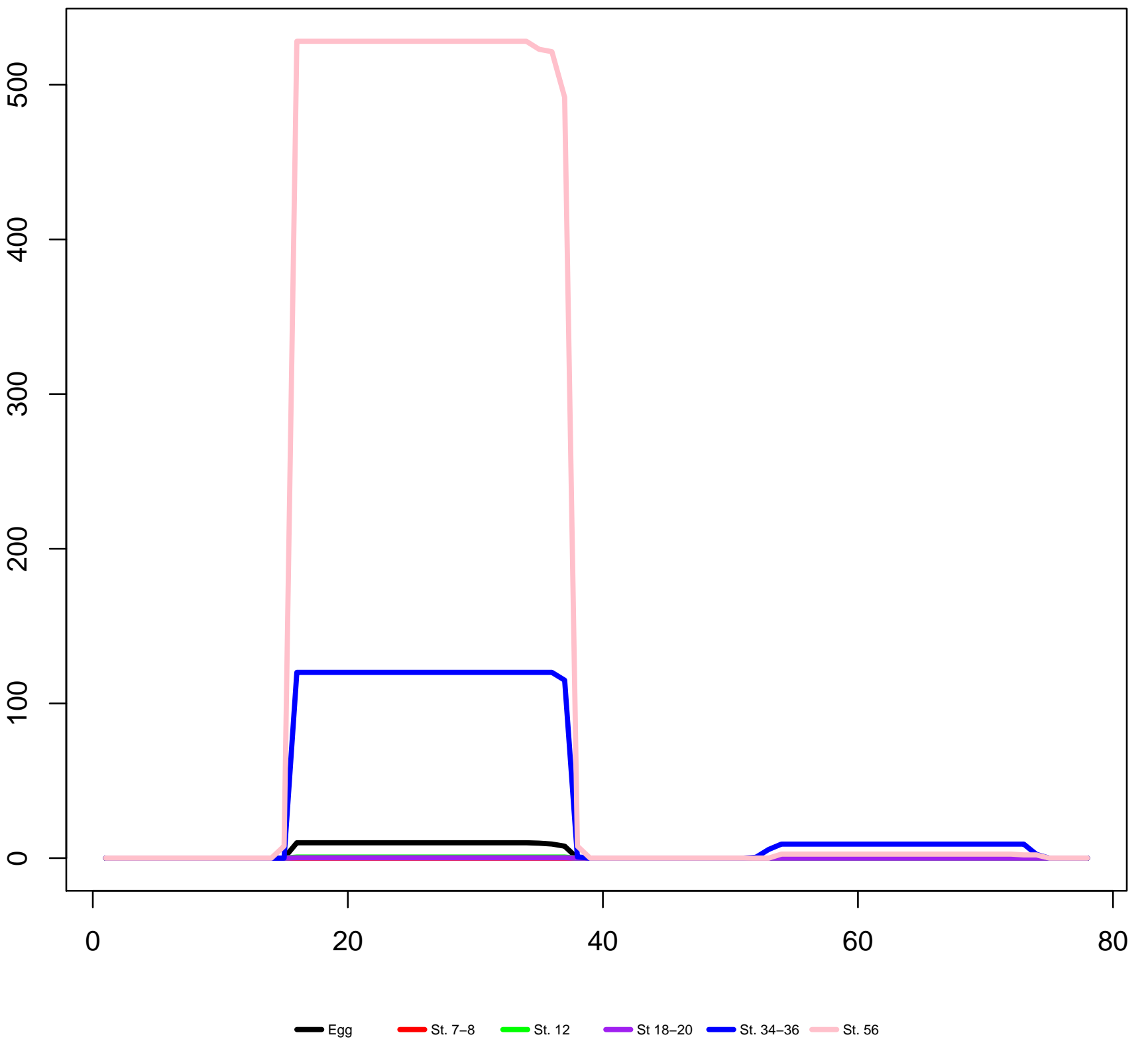


Scaffold18961_923931-924019(+) mir-17

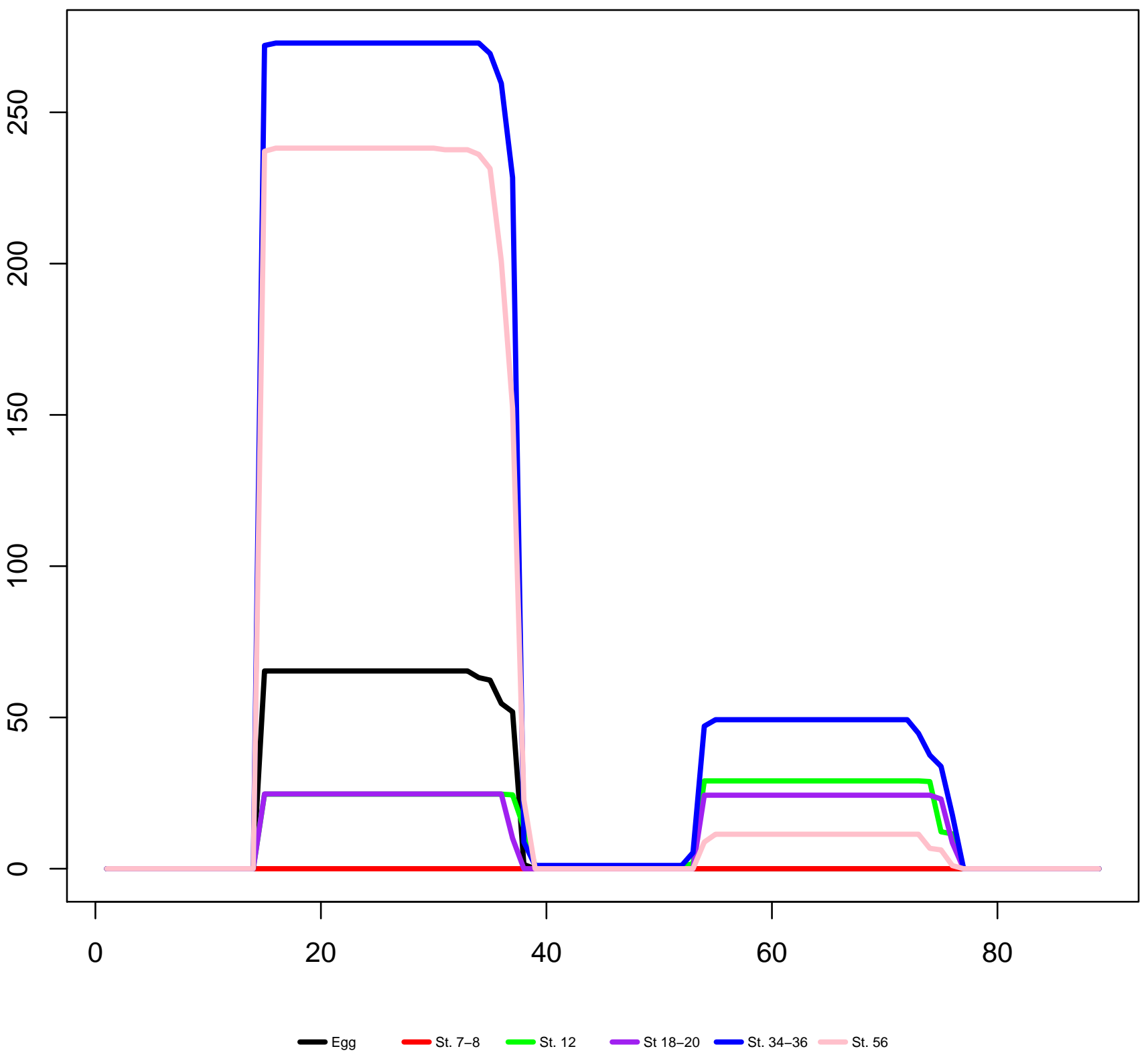


Scaffold18961_924059-924142(+) mir-18a

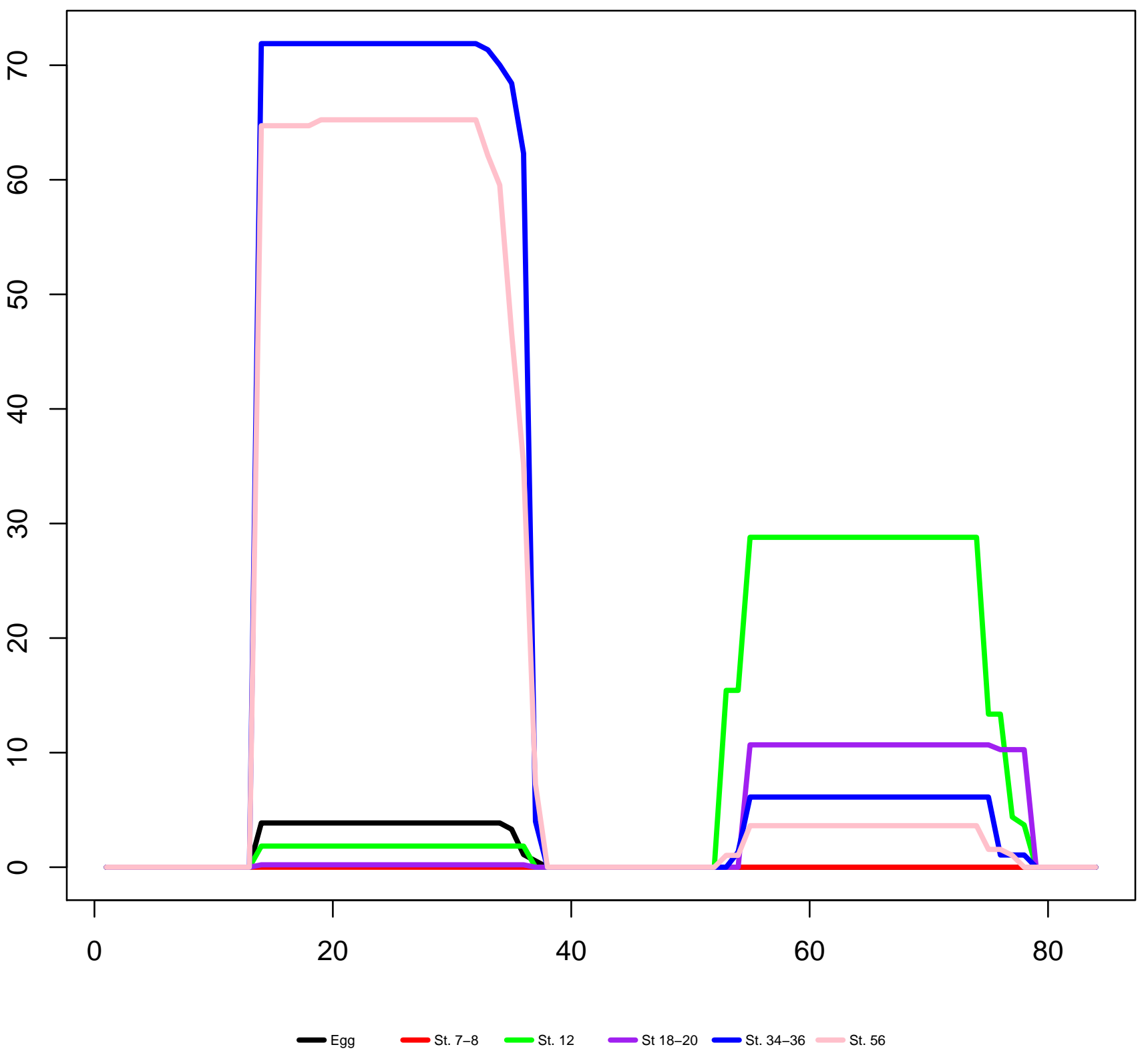


Scaffold18961_924492-924567(+) mir-19b

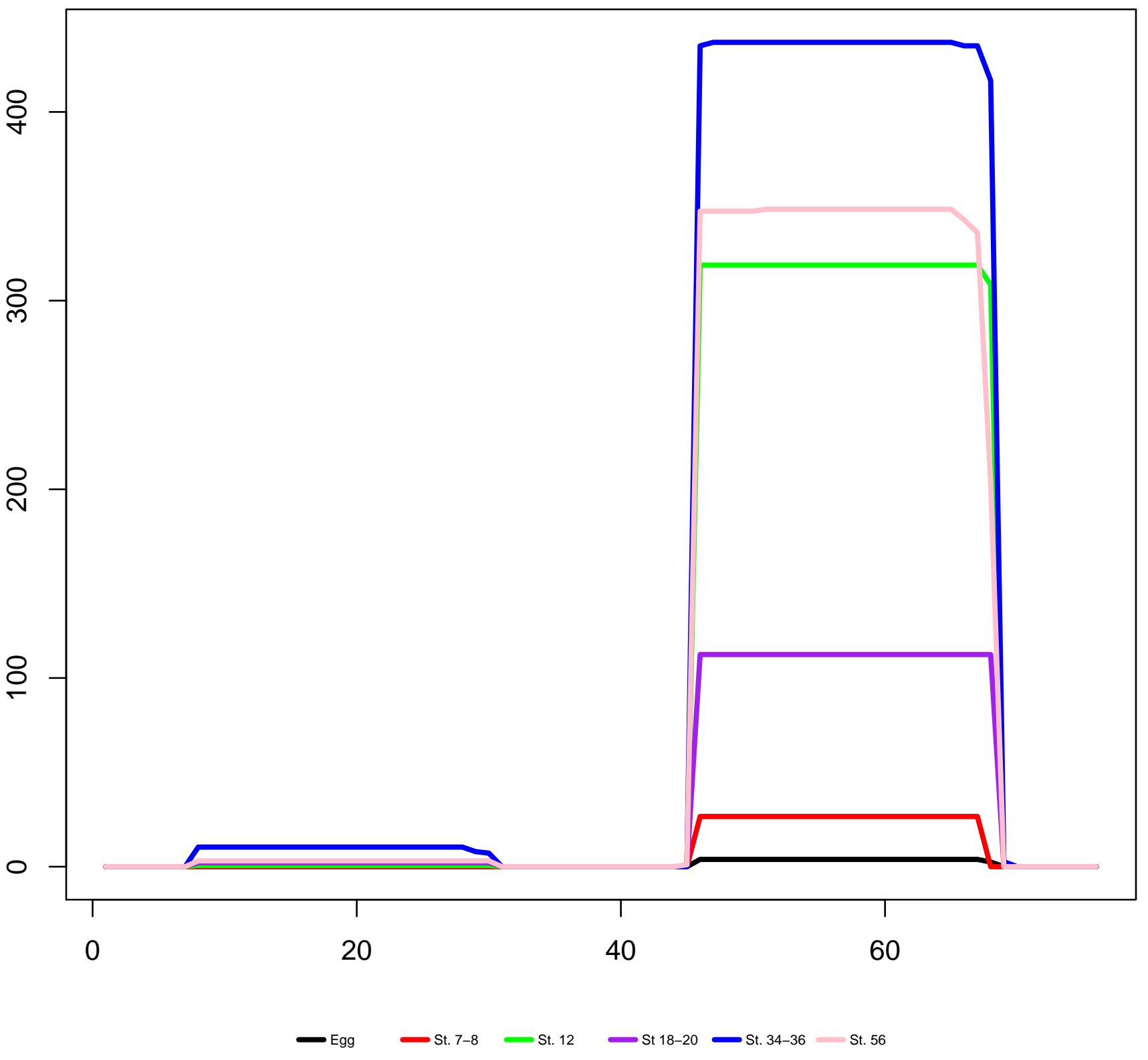


Scaffold191373_11-102(+) mir-458

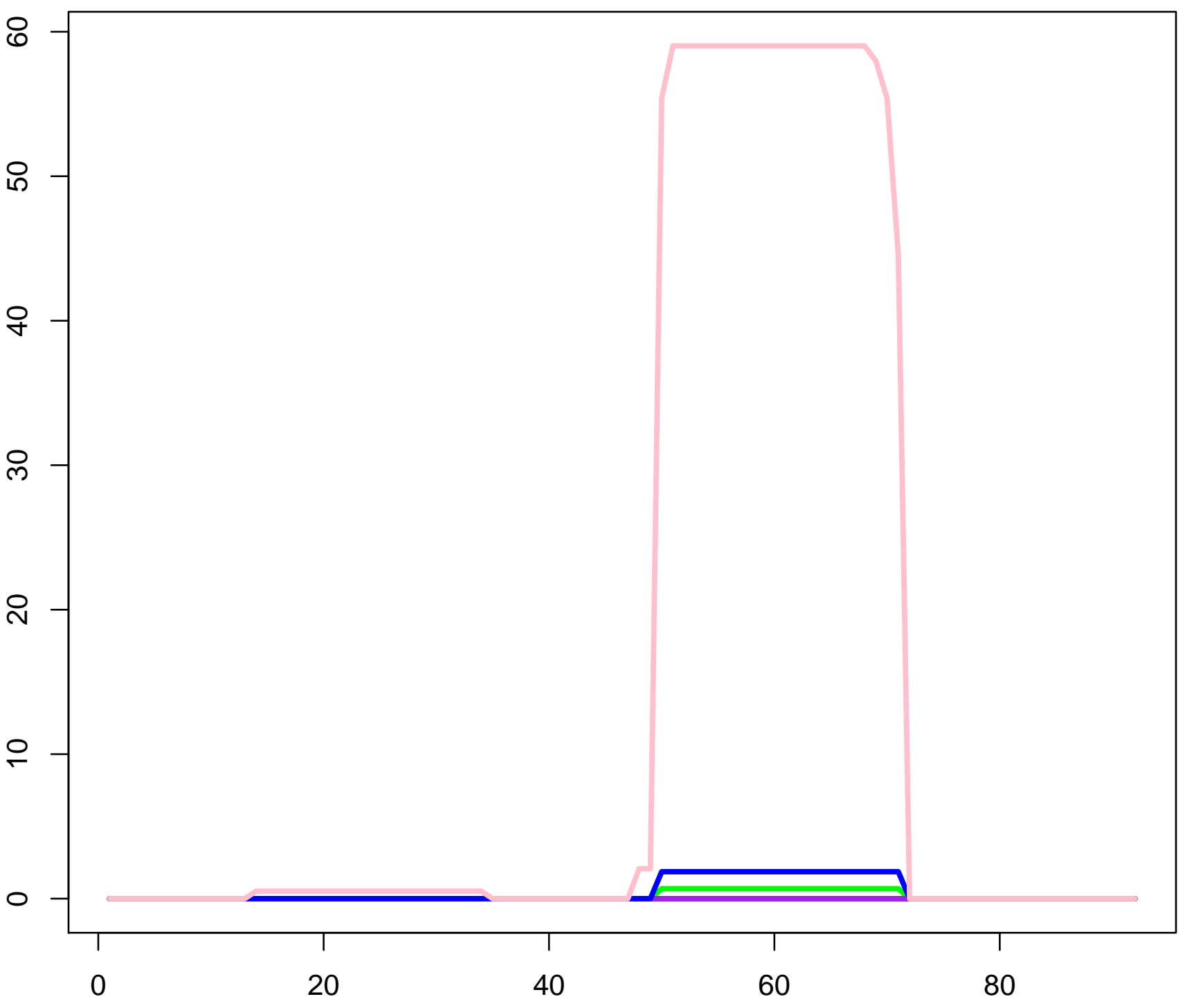


Scaffold19271_640732-640795(-) mir-33a

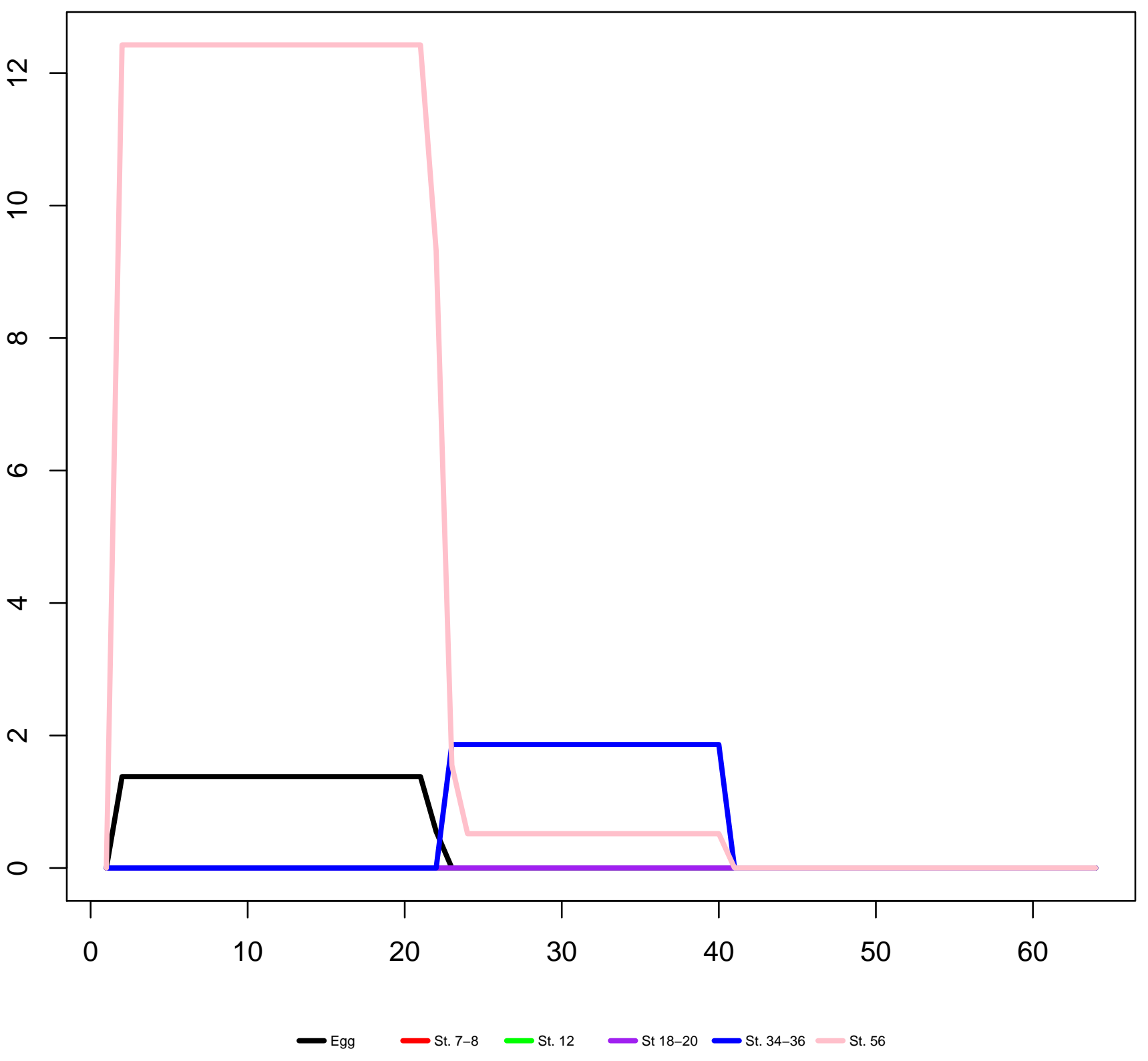


Scaffold19422_10524-10585(-) mir-6236

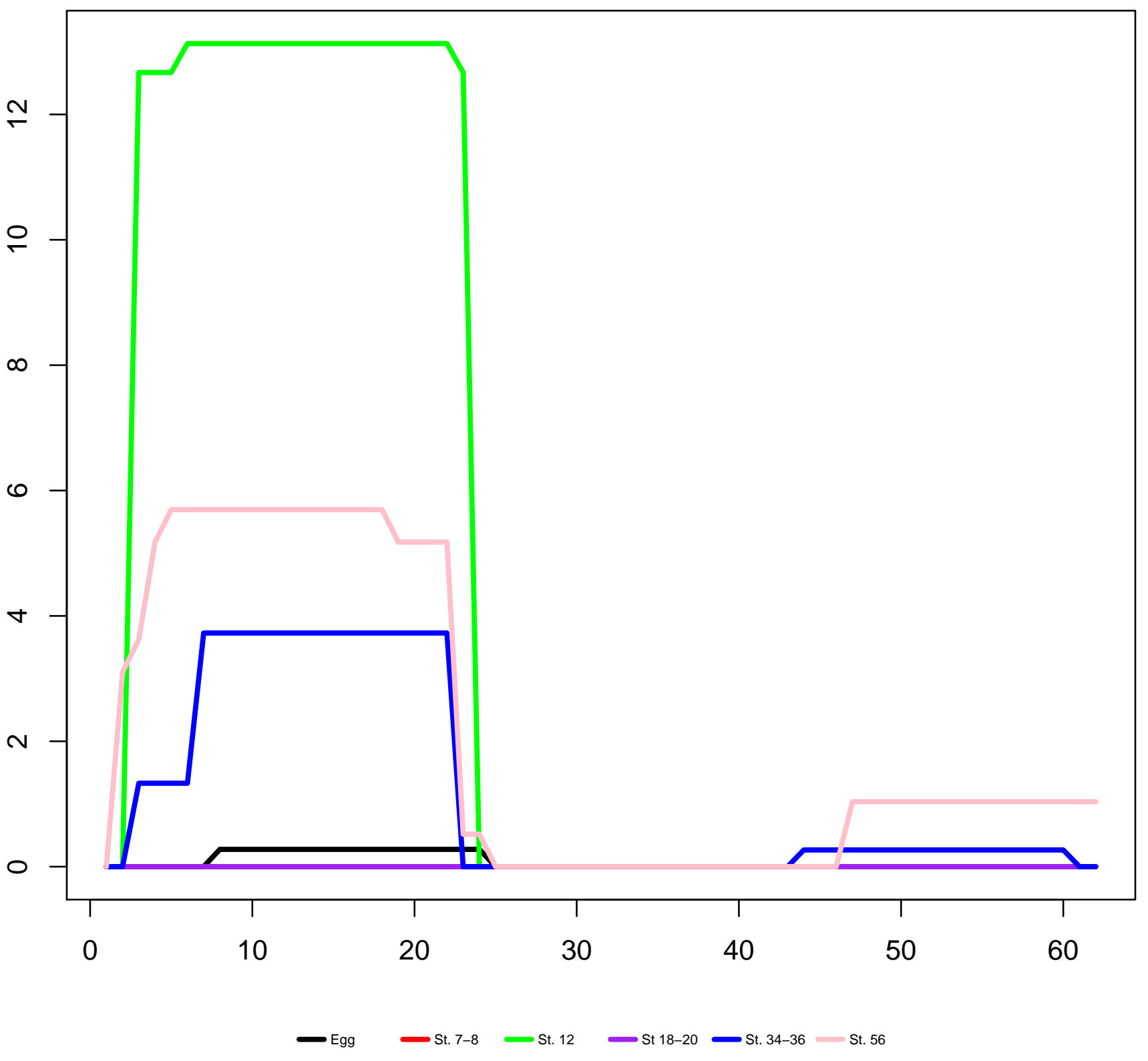


Scaffold20556_1-120(+) mir-218-1

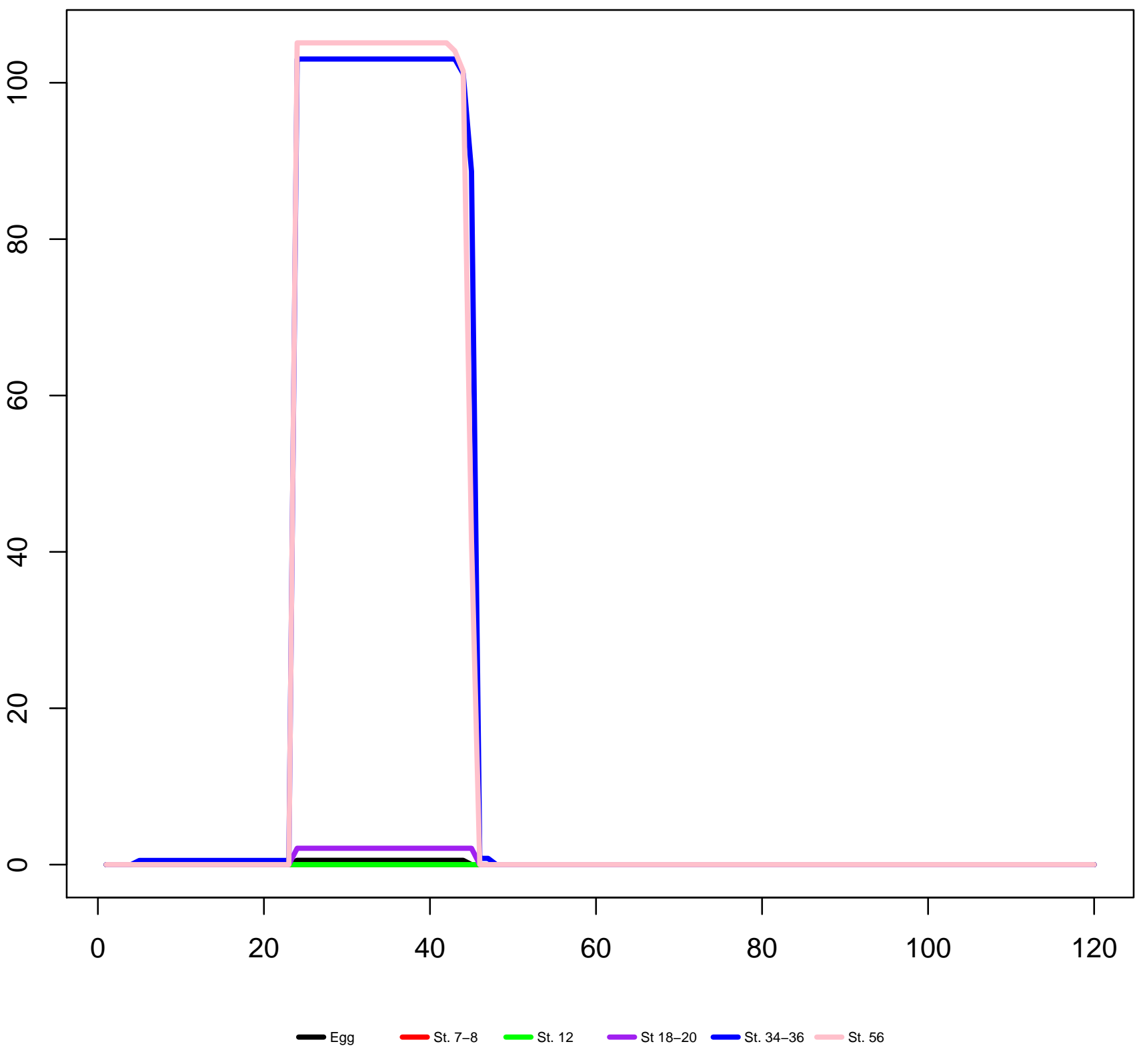


Scaffold20871_437870-437958(+) mir-449c

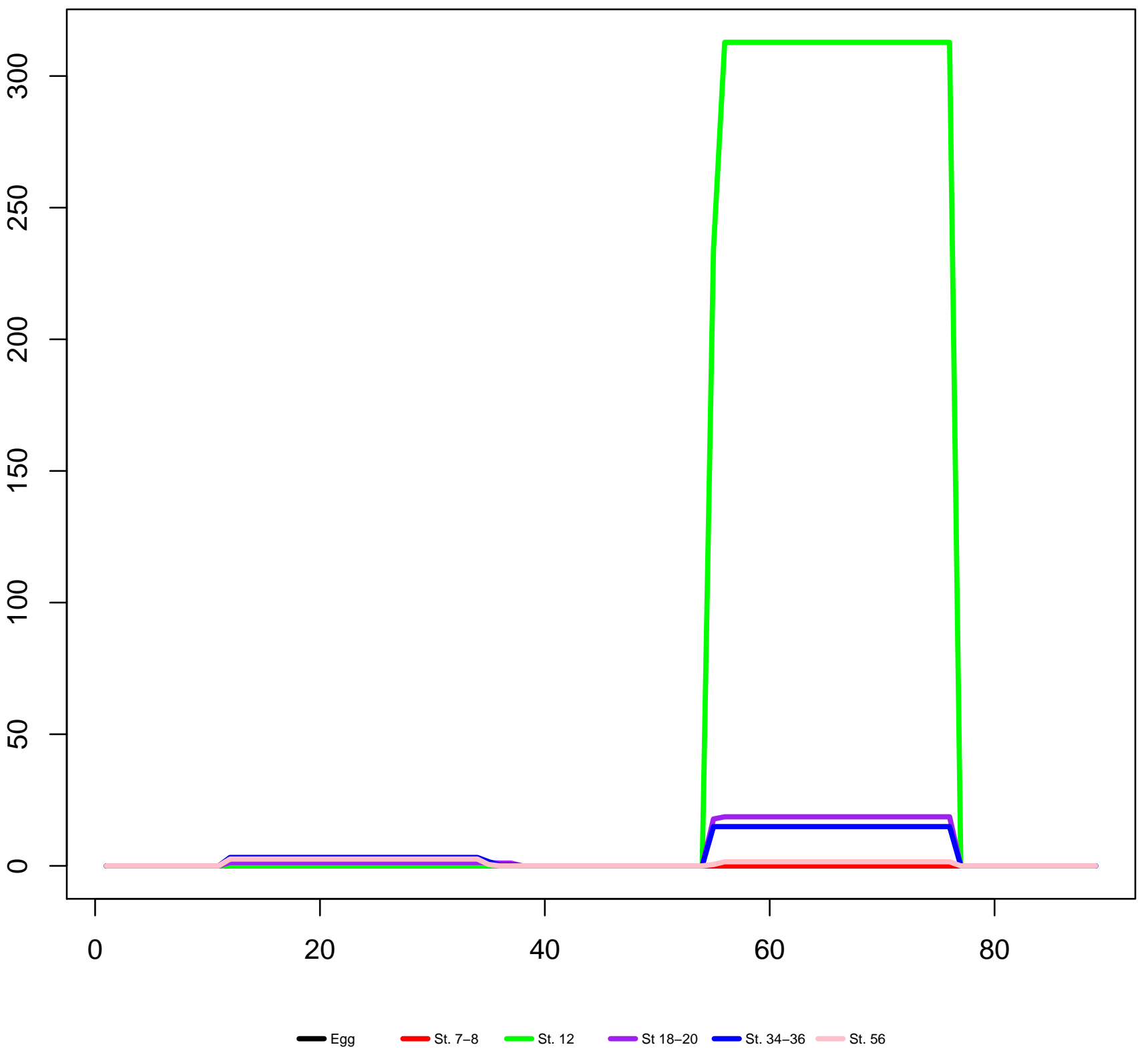




\section{Scaffold20871_438220-438306(+) mir-449a}

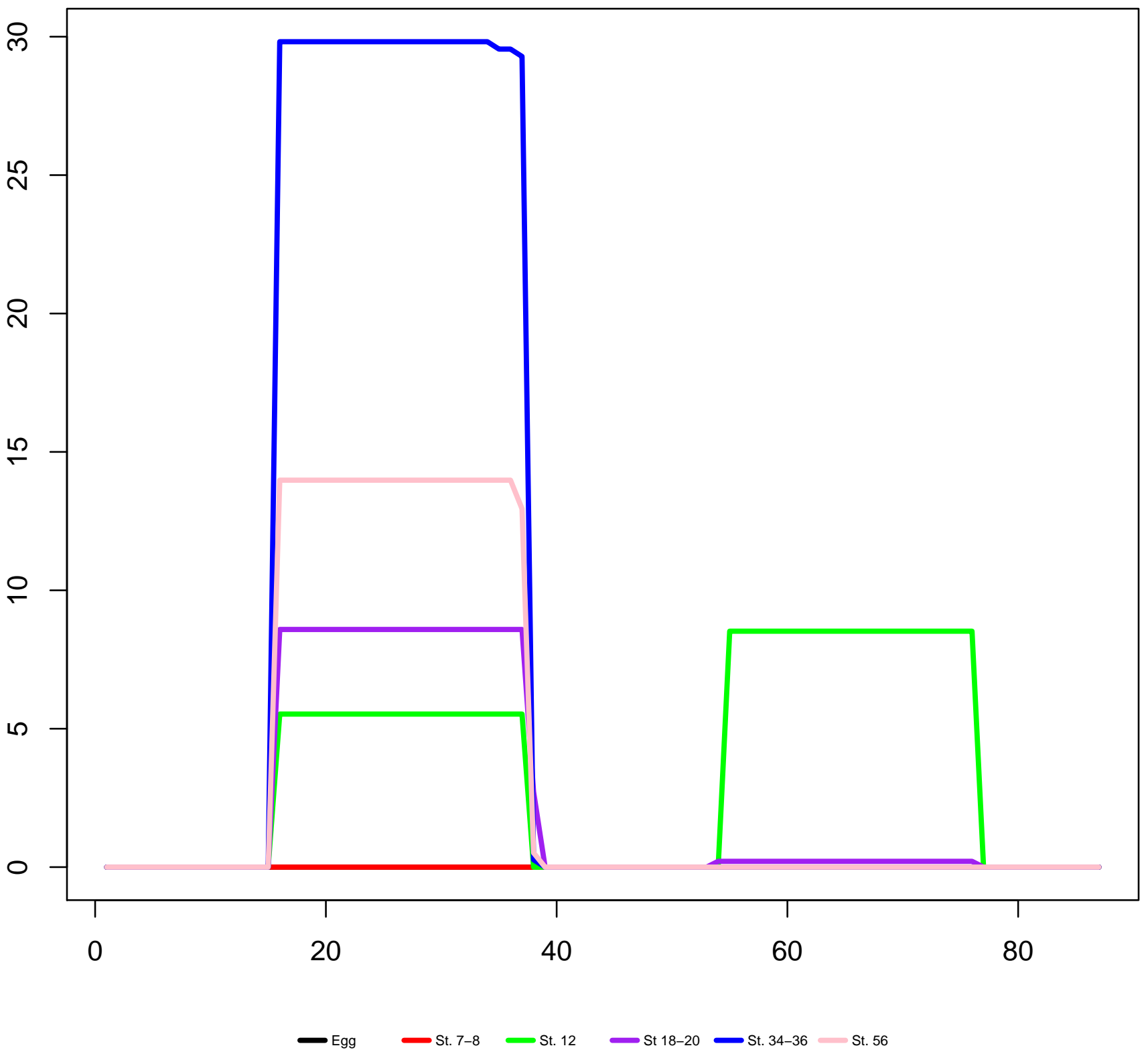


Scaffold21084_309036-309103(+) mir-375

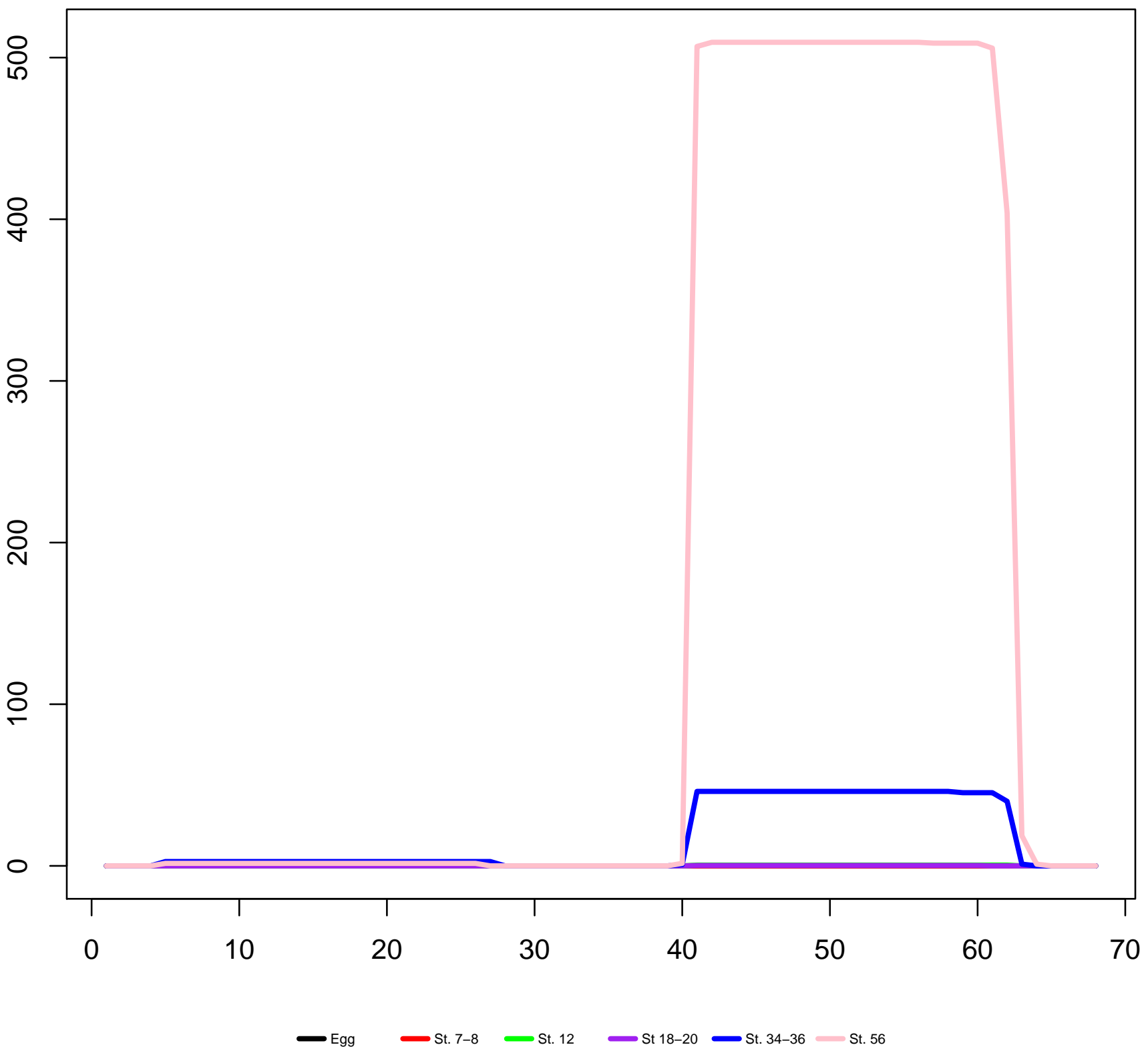


Scaffold217787_119667-119732(-) mir-222a

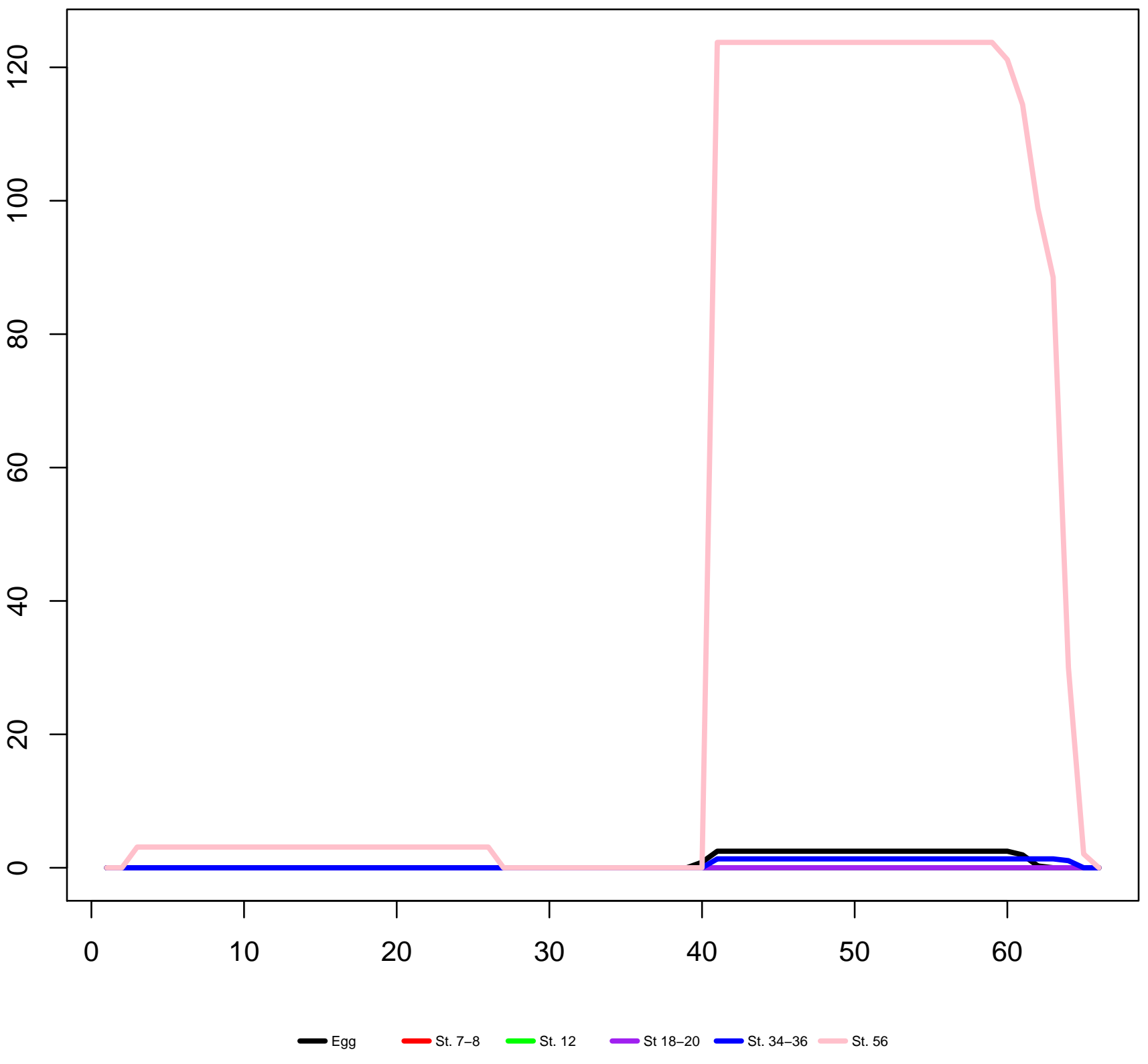


Scaffold224722_61896-61992(+) let-7a

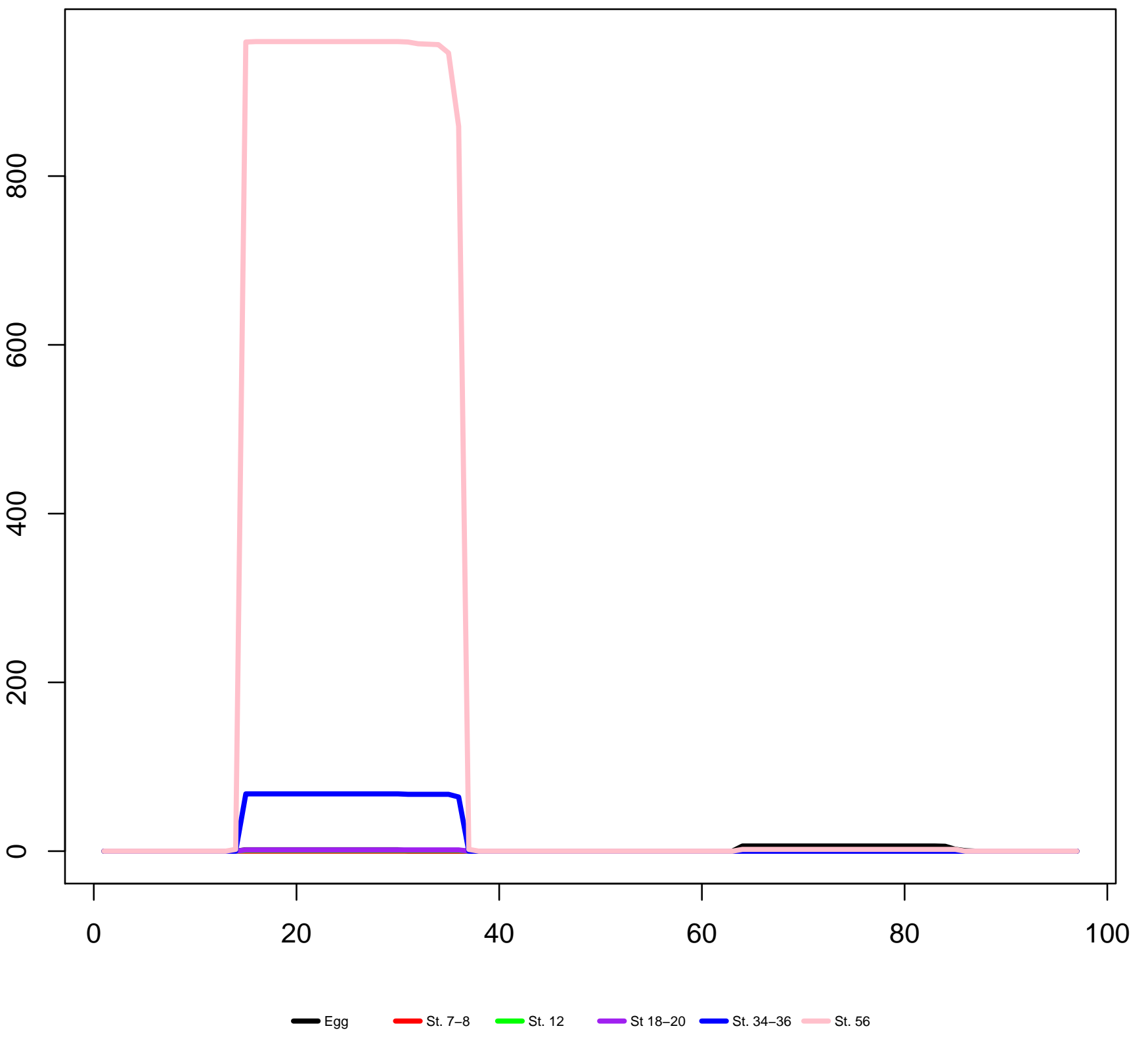


Scaffold224722_62244-62330(+) let-7b

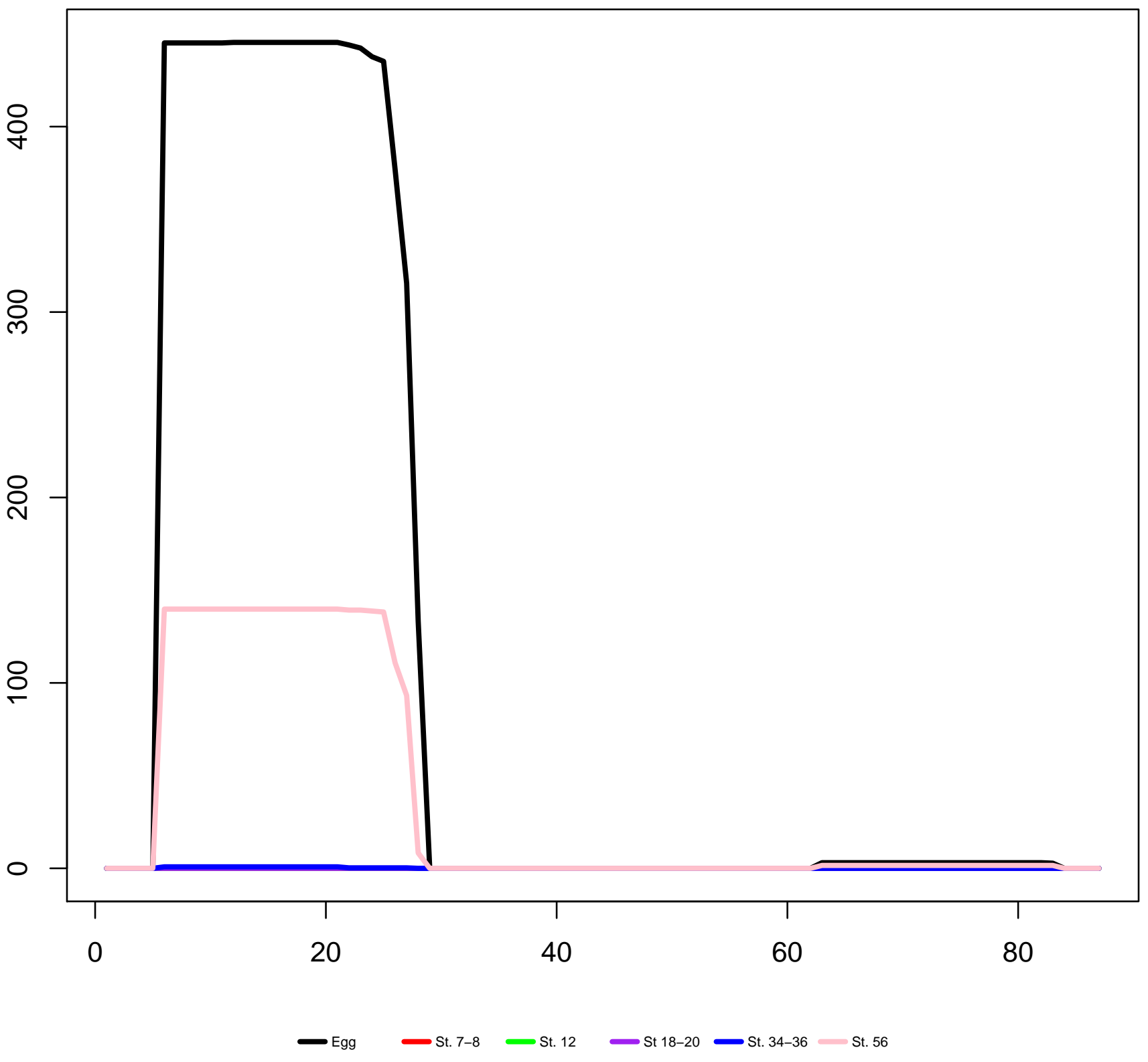


Scaffold23022_556474-556601(+) mir-218-1

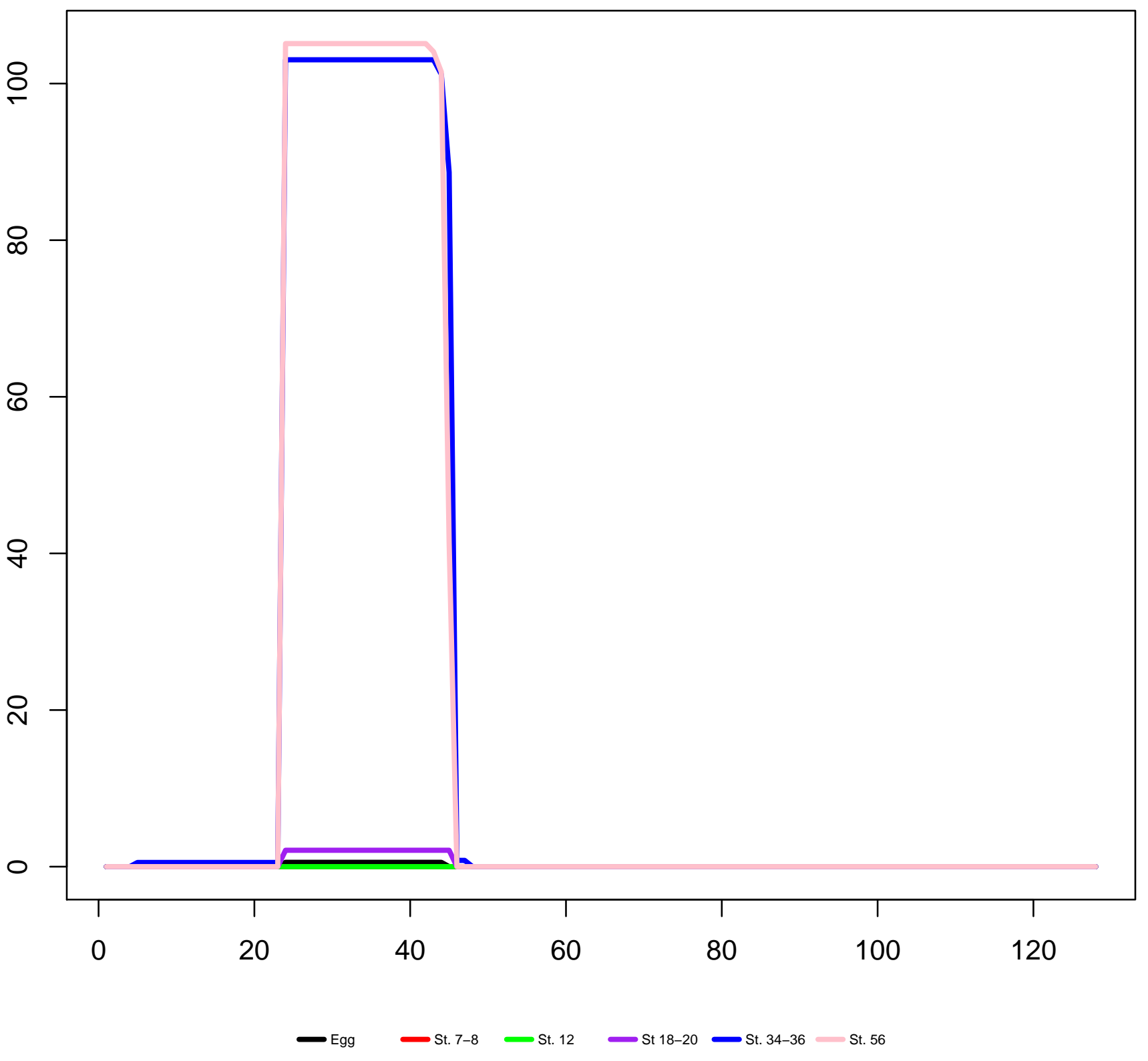


Scaffold23207_724652-724733(-) mir-128-2

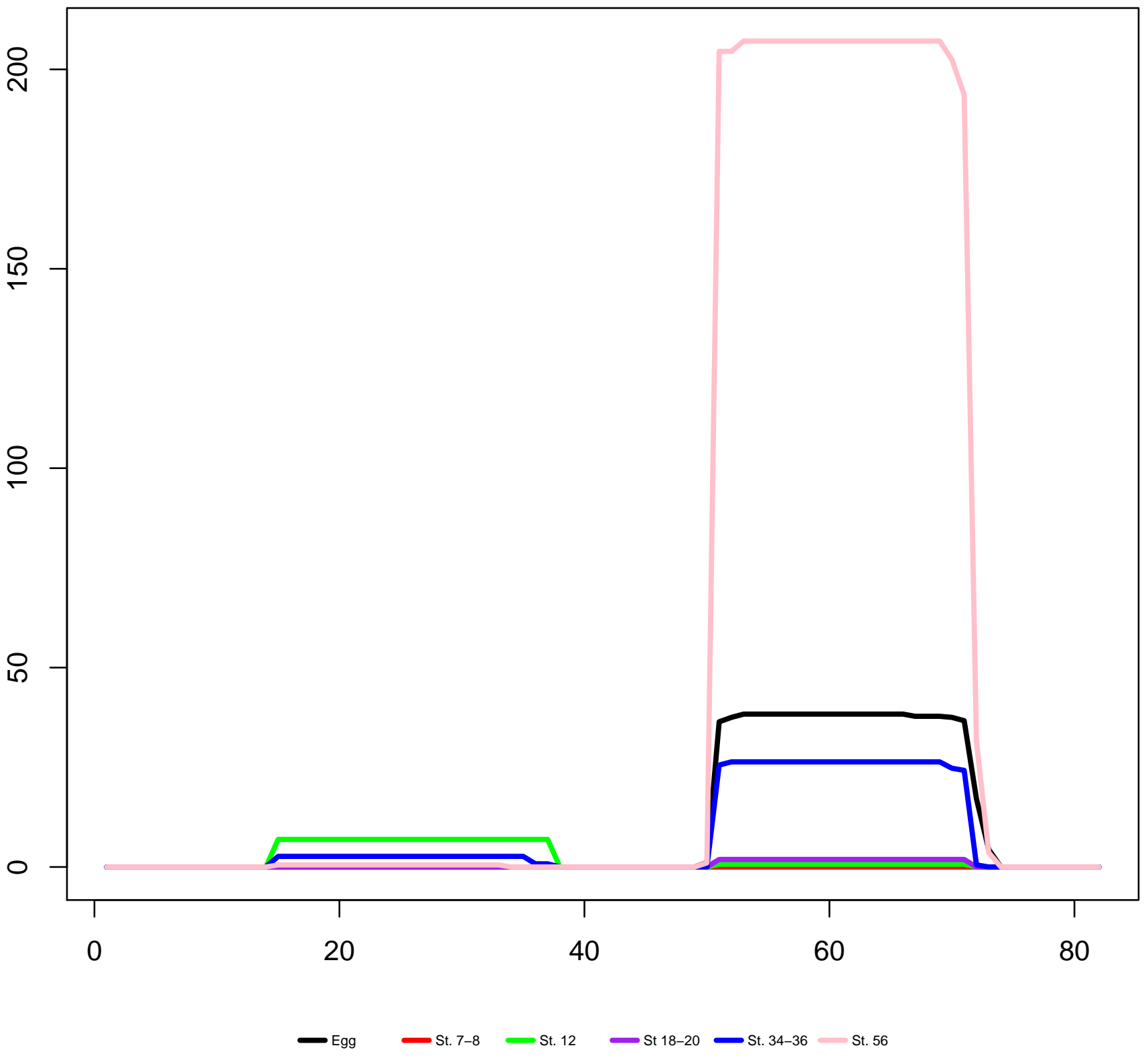


Scaffold23952_1608154-1608245(+) mir-458

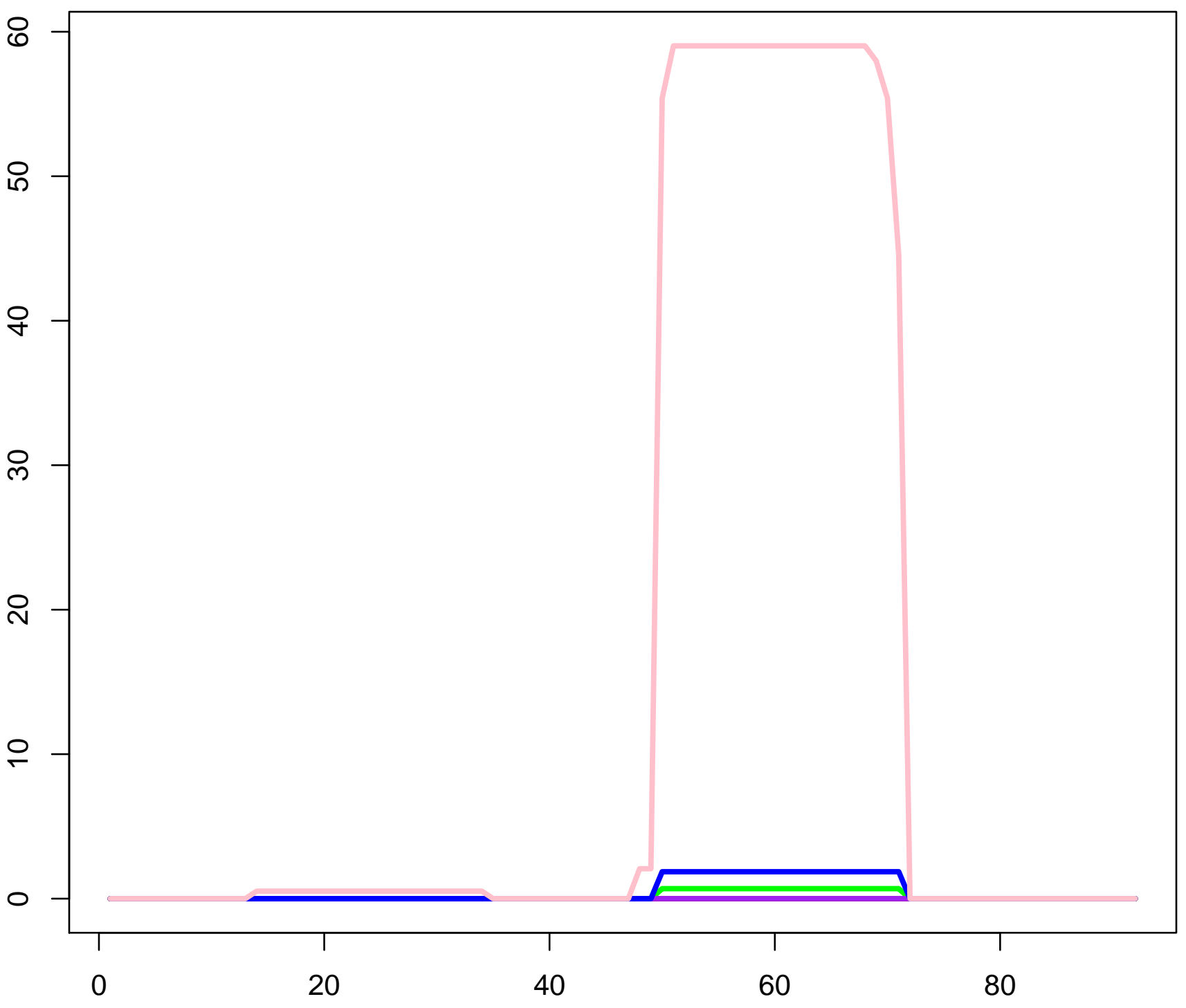


Scaffold244529_1-95(-) mir-125b-1

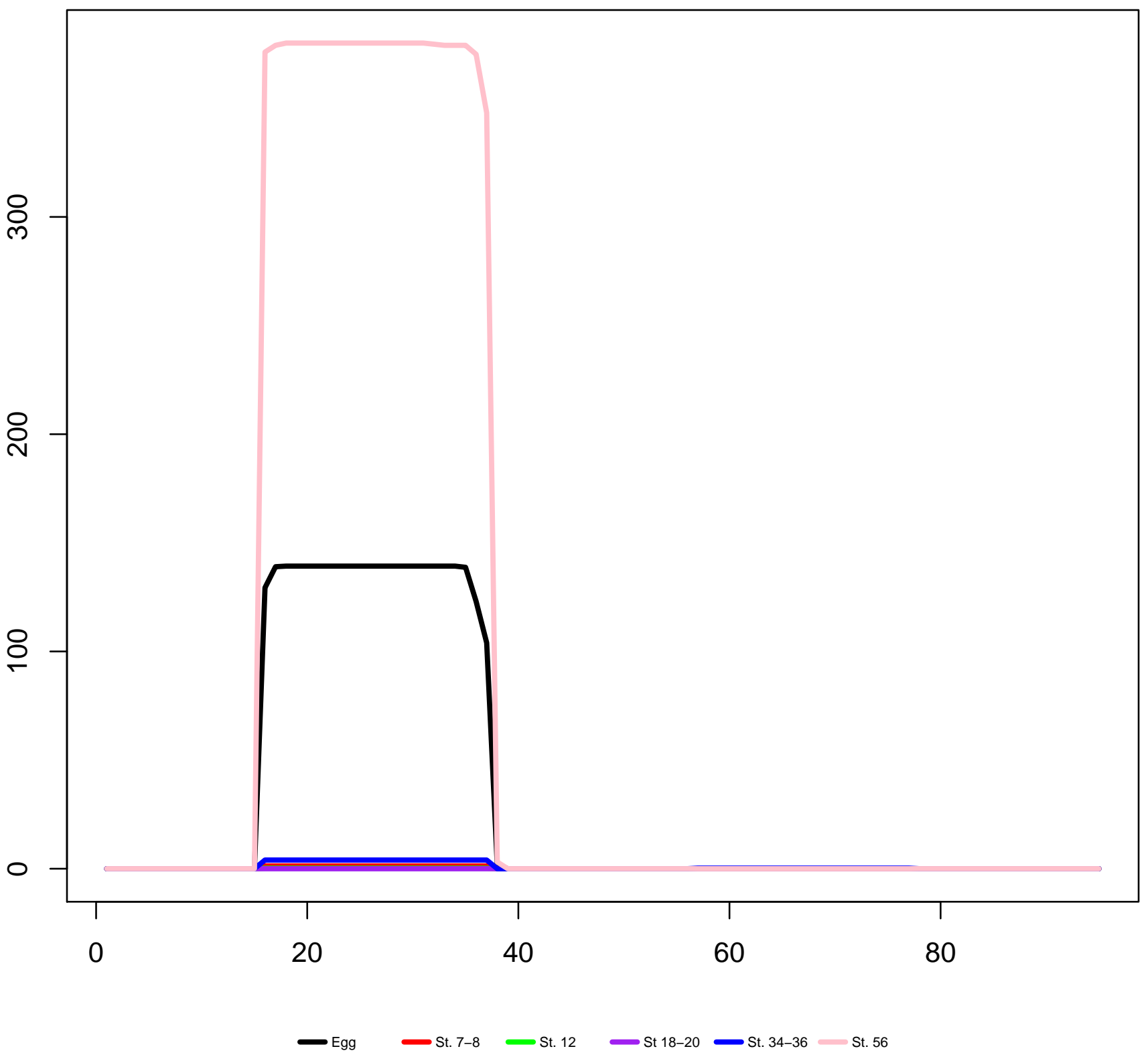


Scaffold246100_95140-95227(-) mir-489

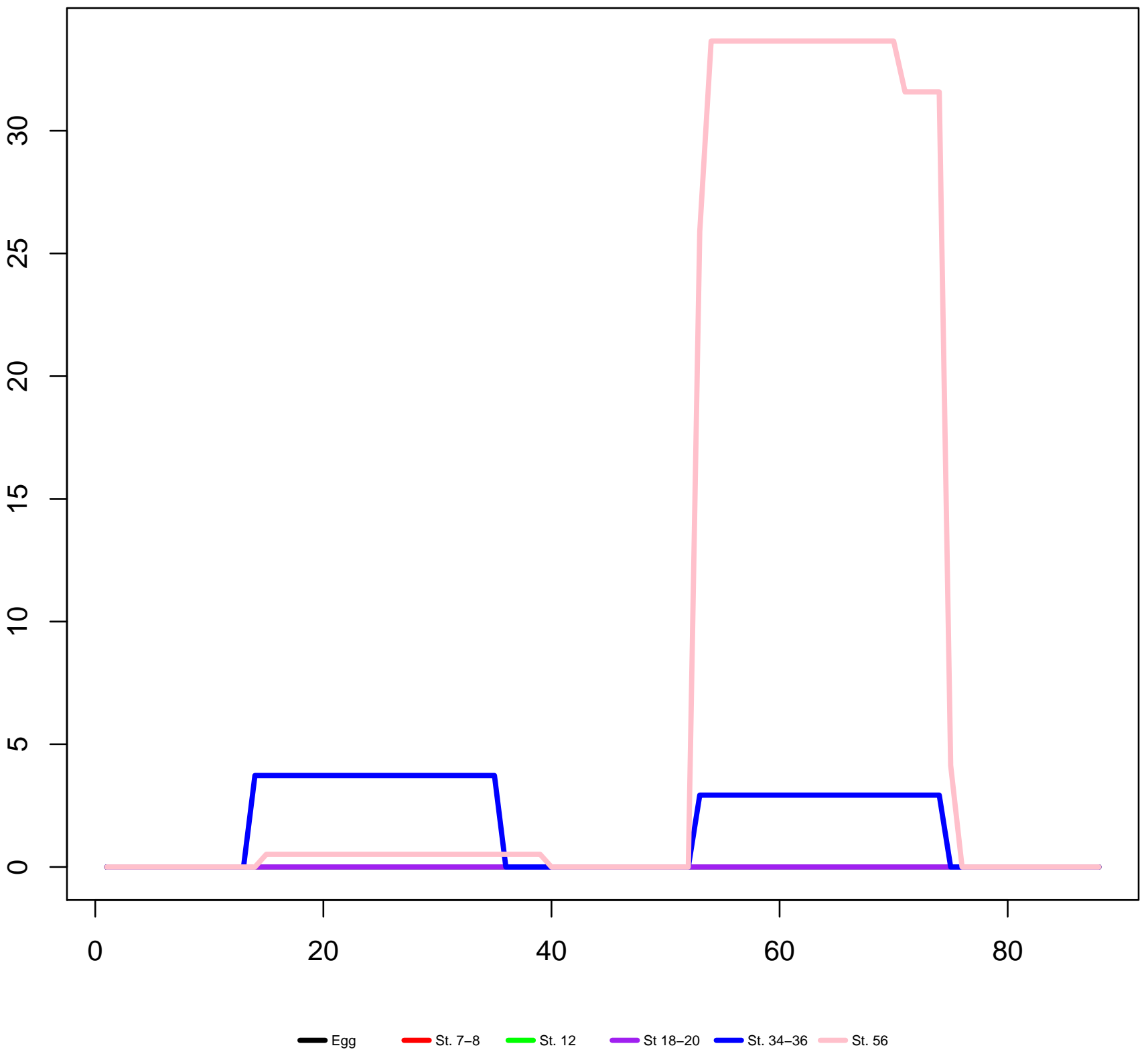


Scaffold24919_270362-270434(+) mir-1662

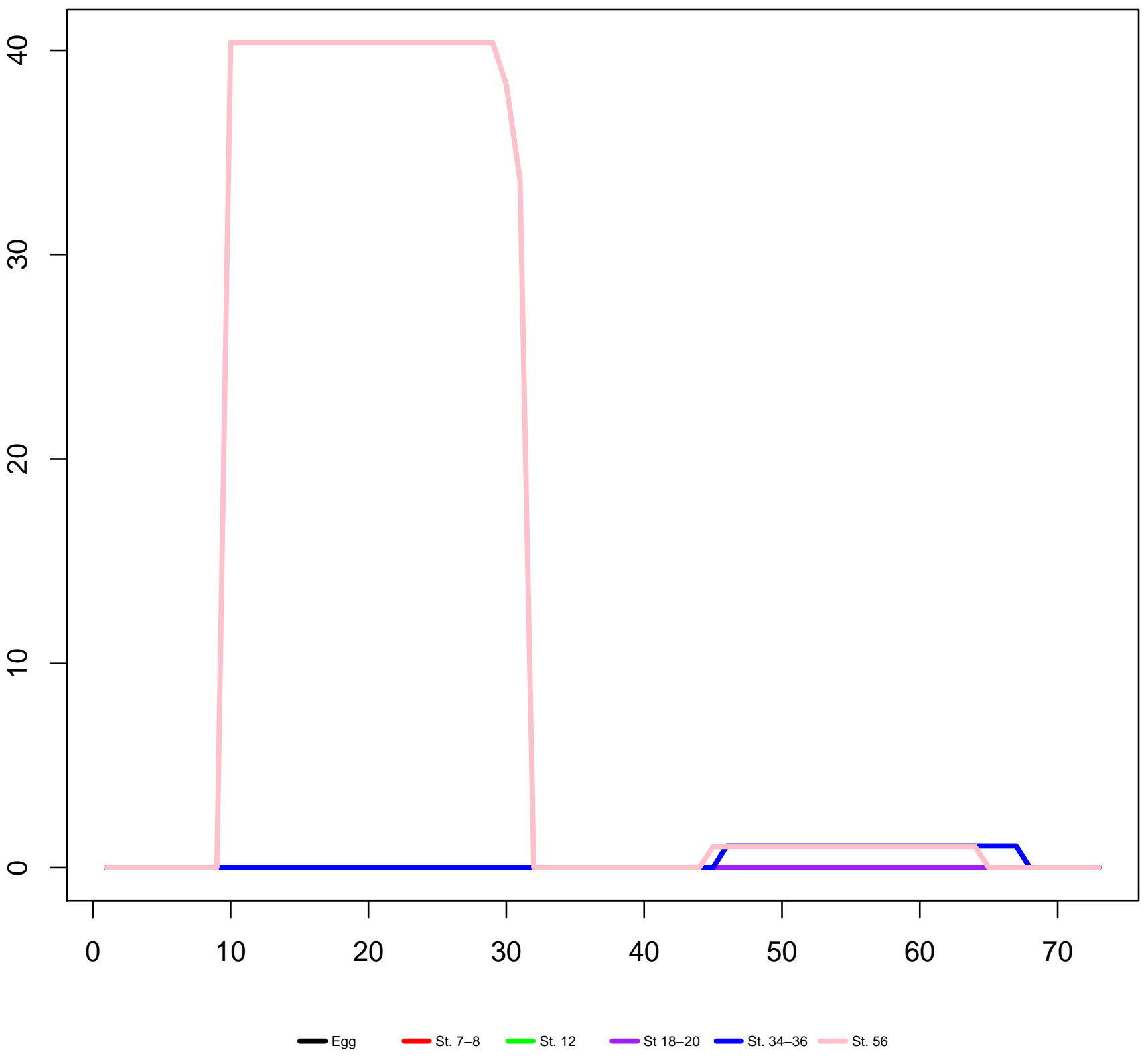


Scaffold250320_5-115(-) let-7c-1

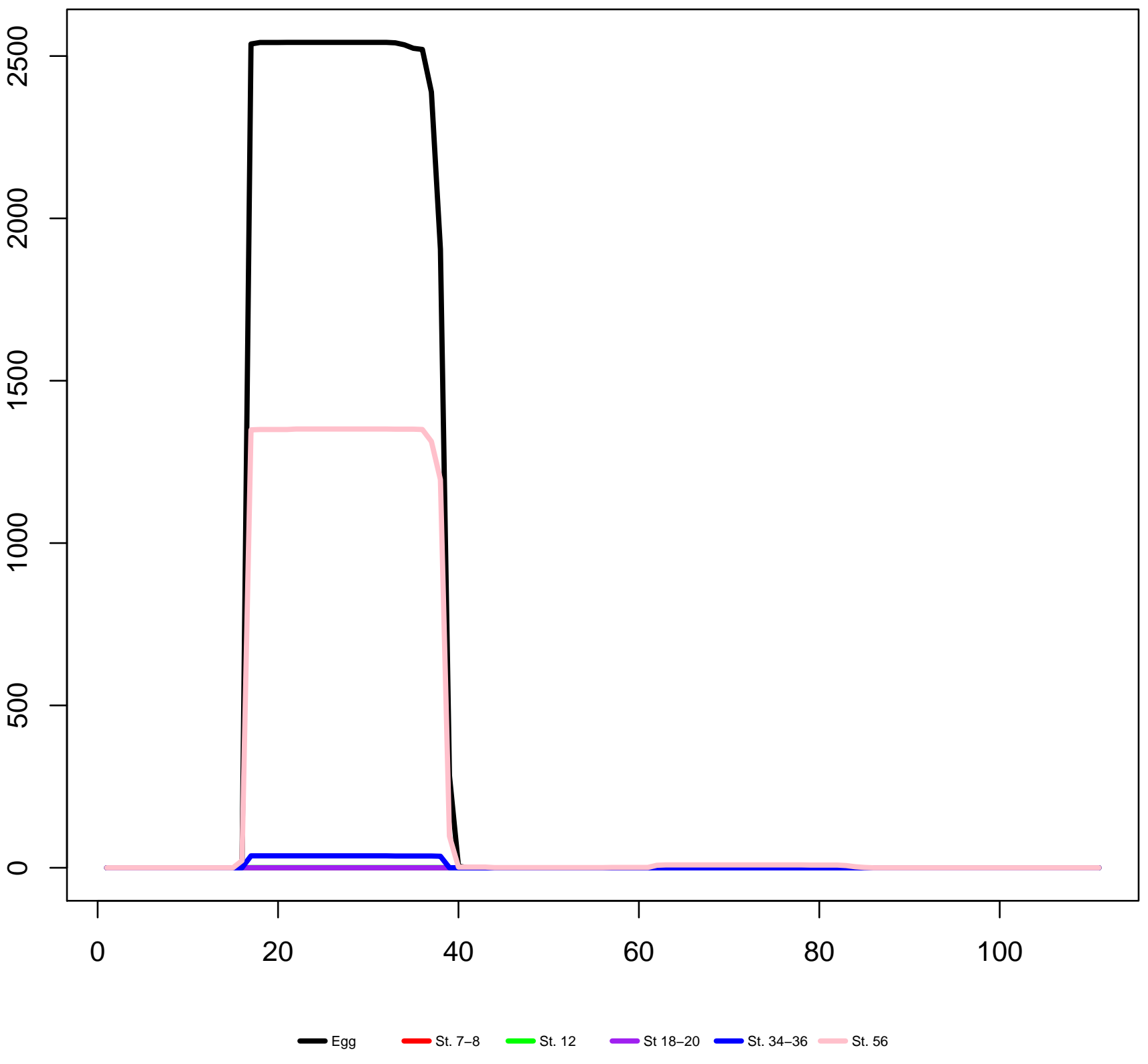


Scaffold25792_331869-331938(+) mir-206

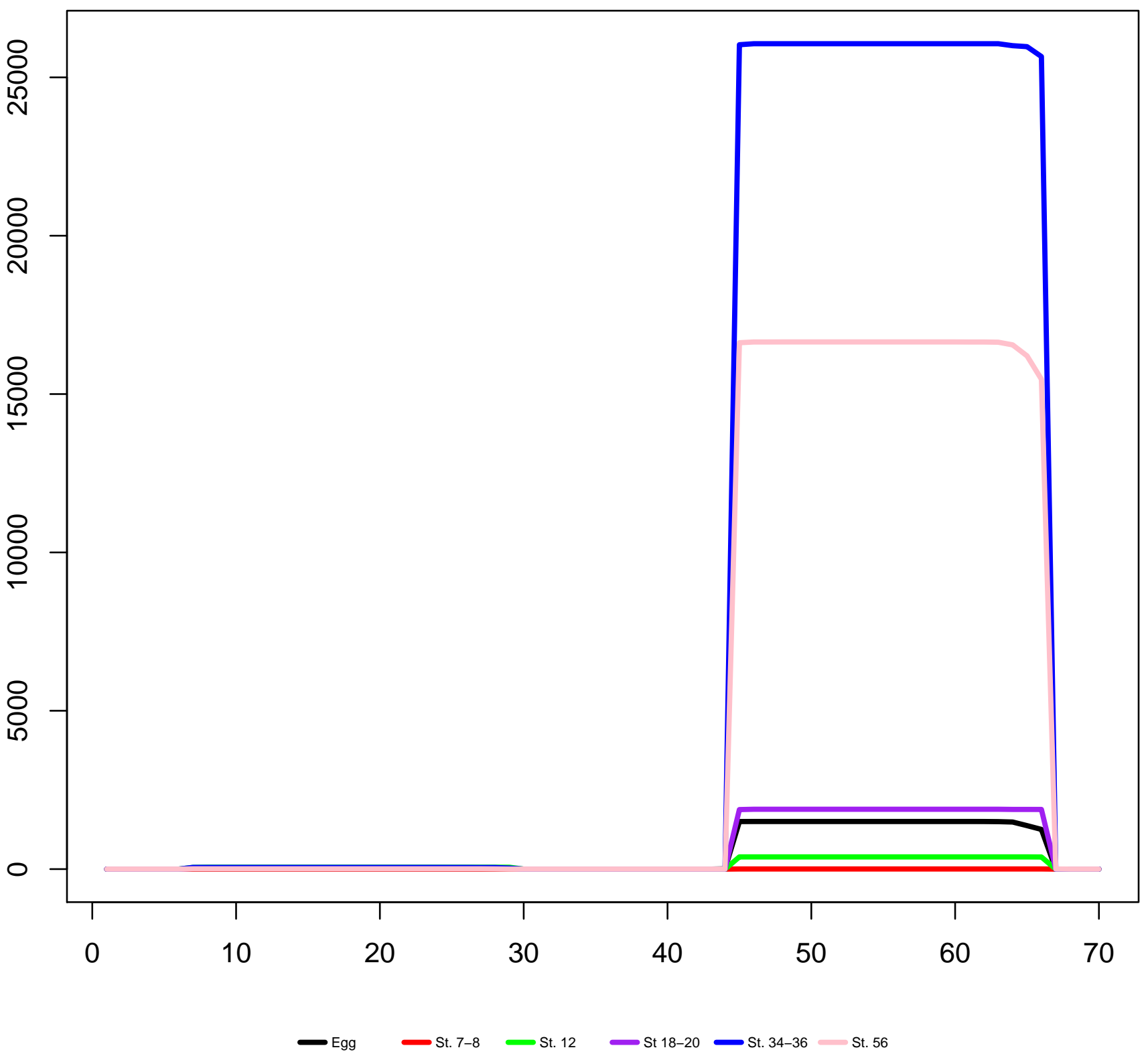




\section{Scaffold259001_138256-138341(+) mir-499}

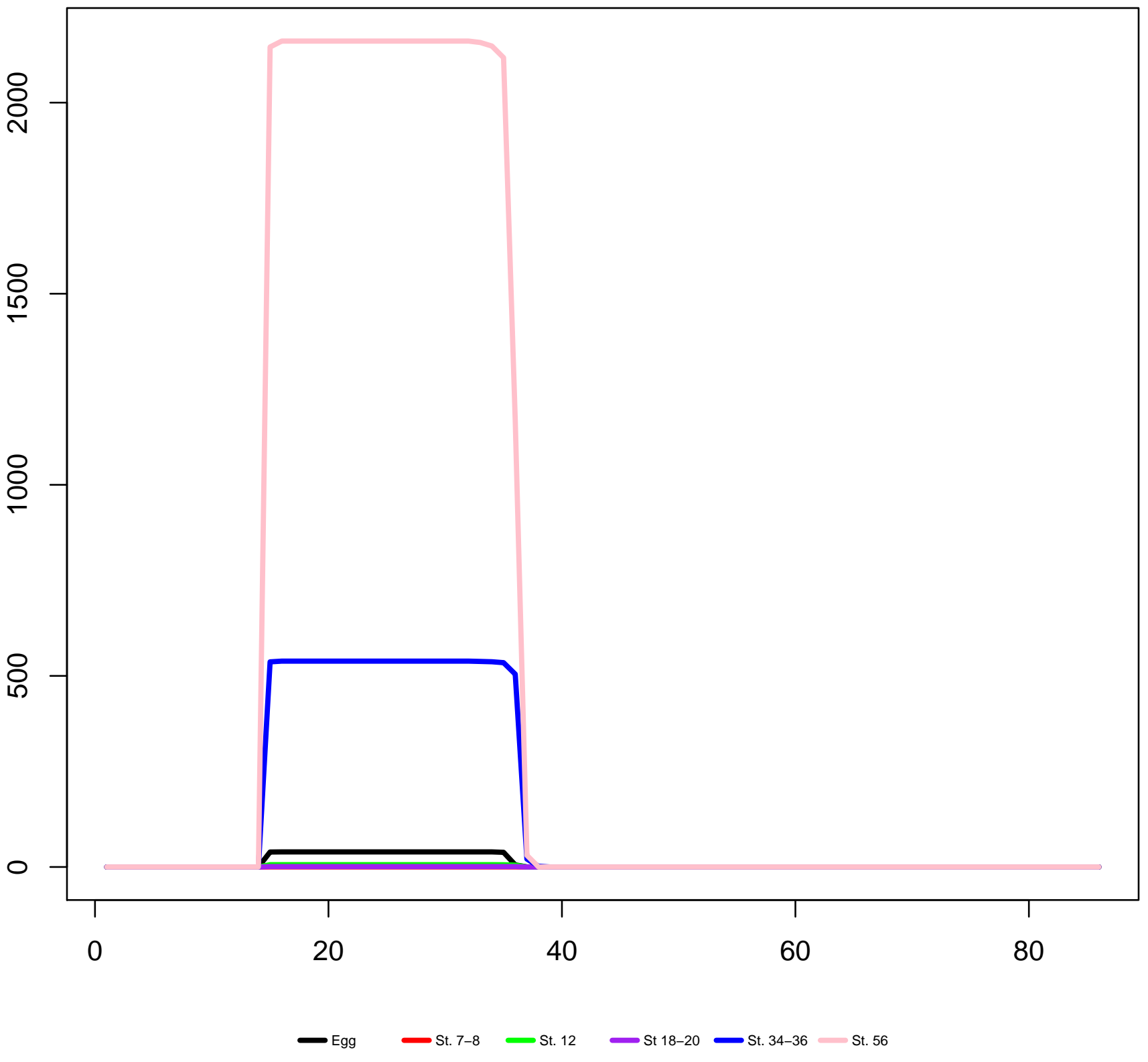


Scaffold259104_105101-105178(+) let-7b

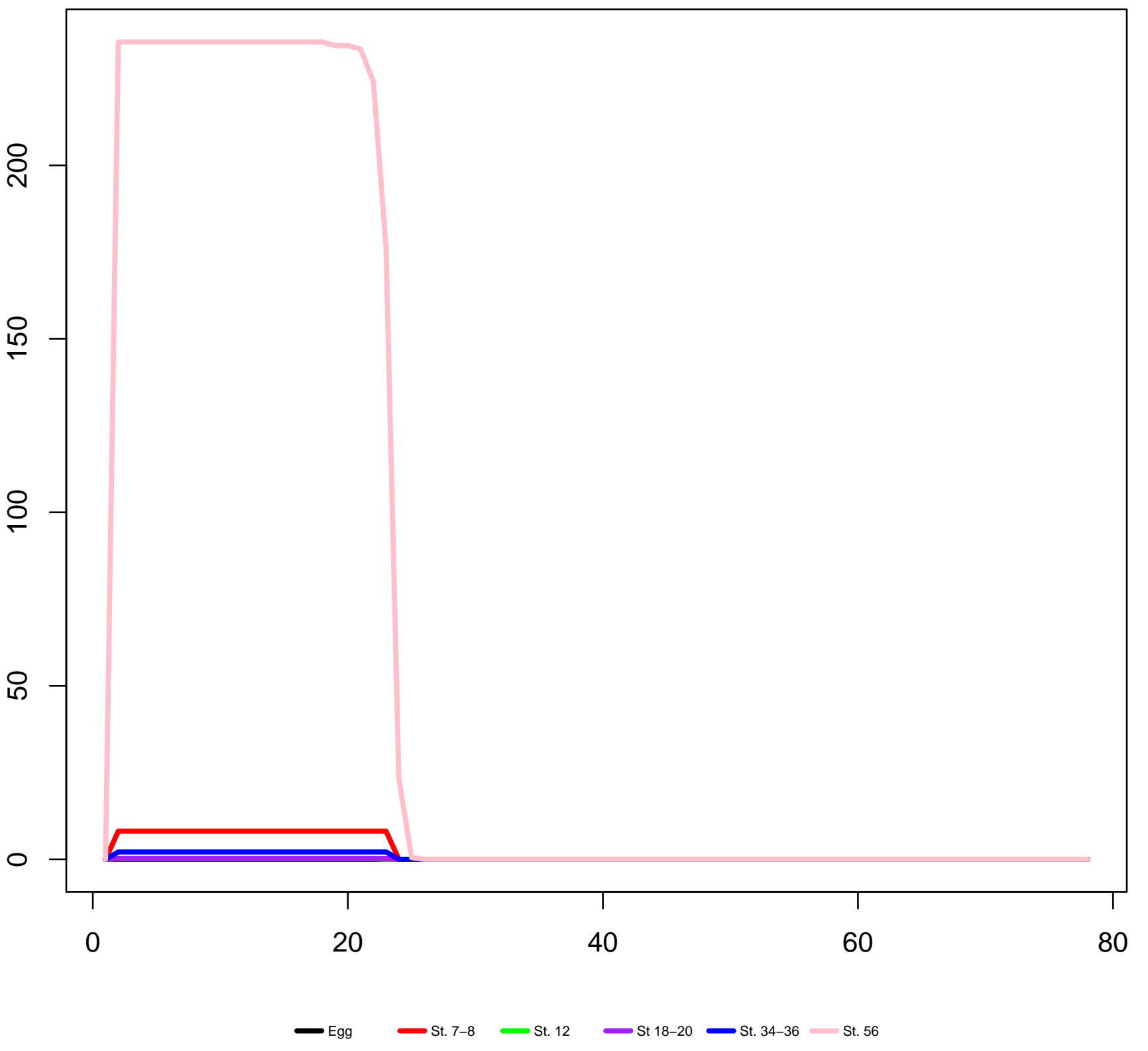


Scaffold260860_20-89(-) mir-301b

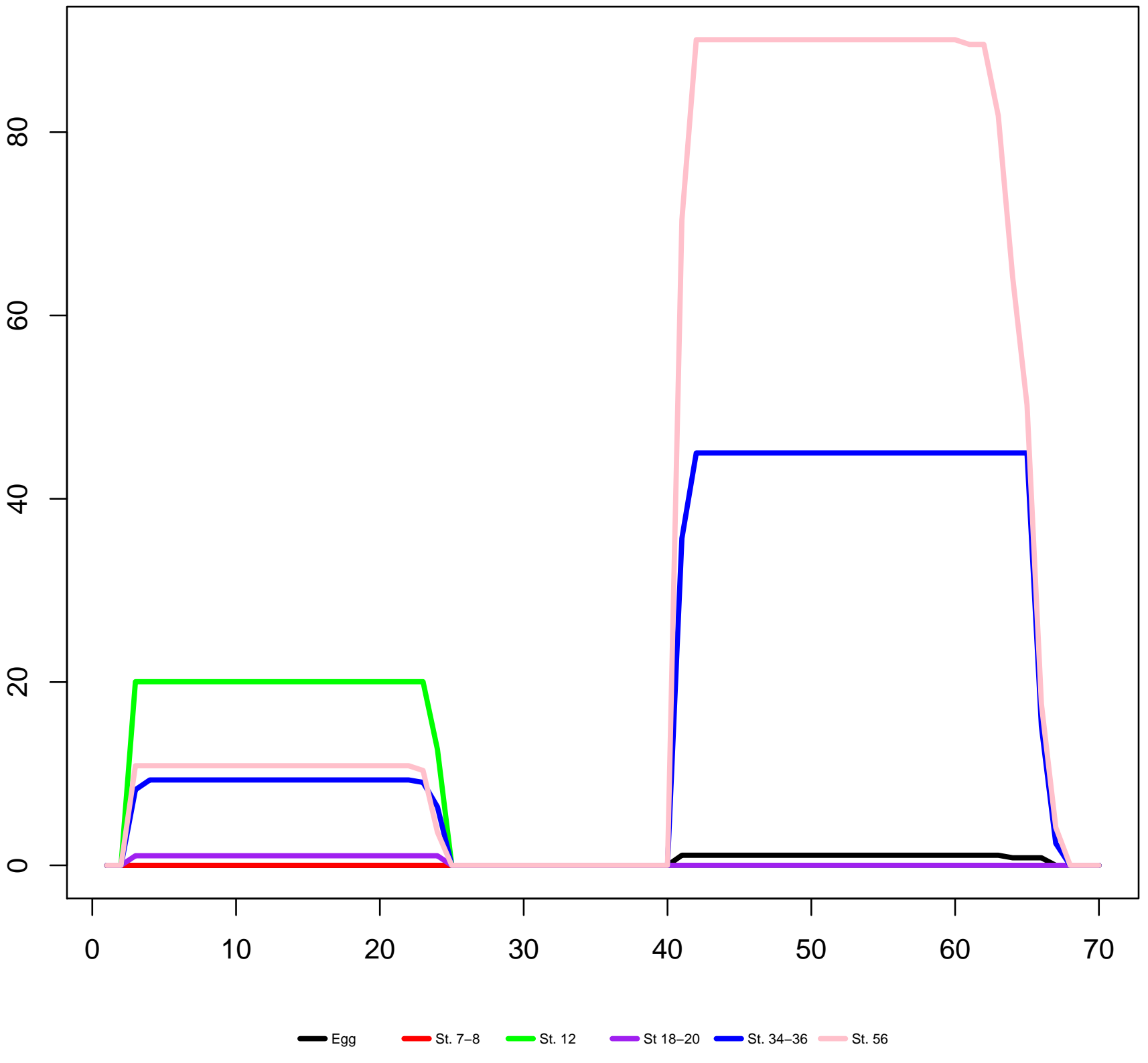


Scaffold26457_67668-67757(-) mir-29a-2

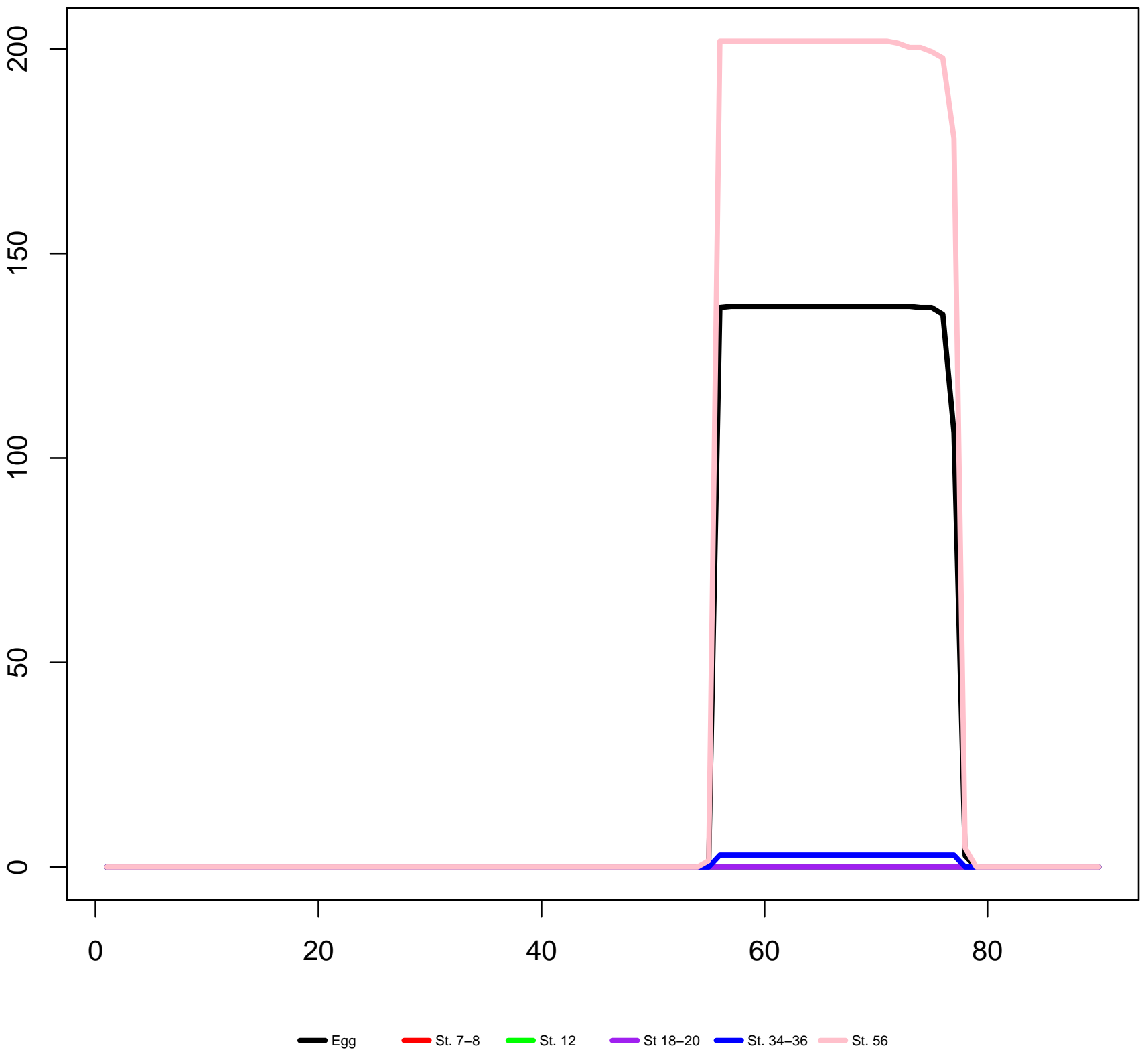


Scaffold26496_341785-341885(-) mir-199b

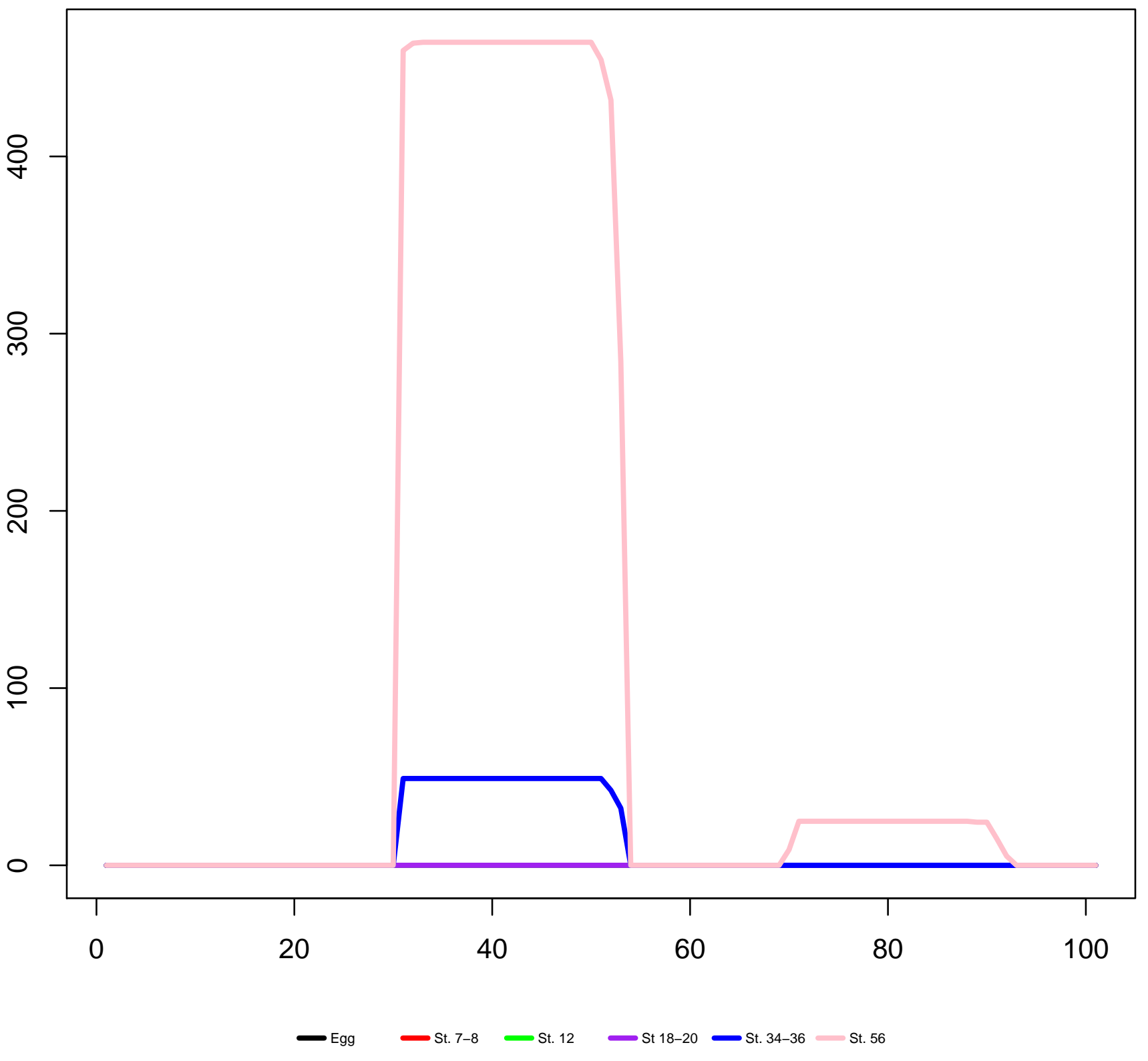


Scaffold27158_37704-37771(+) mir-129b

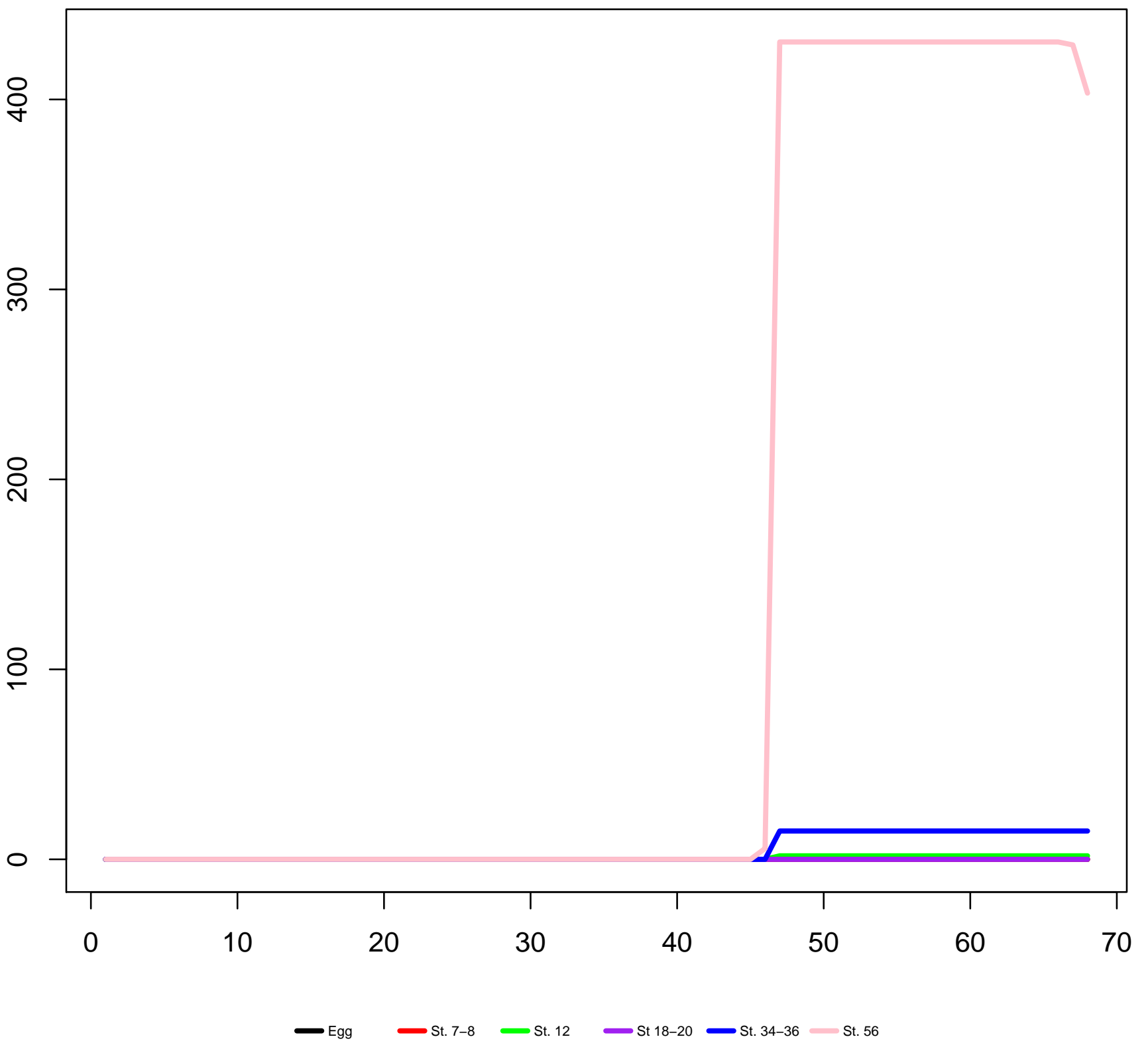


Scaffold27441_314220-314325(+) mir-199a-1

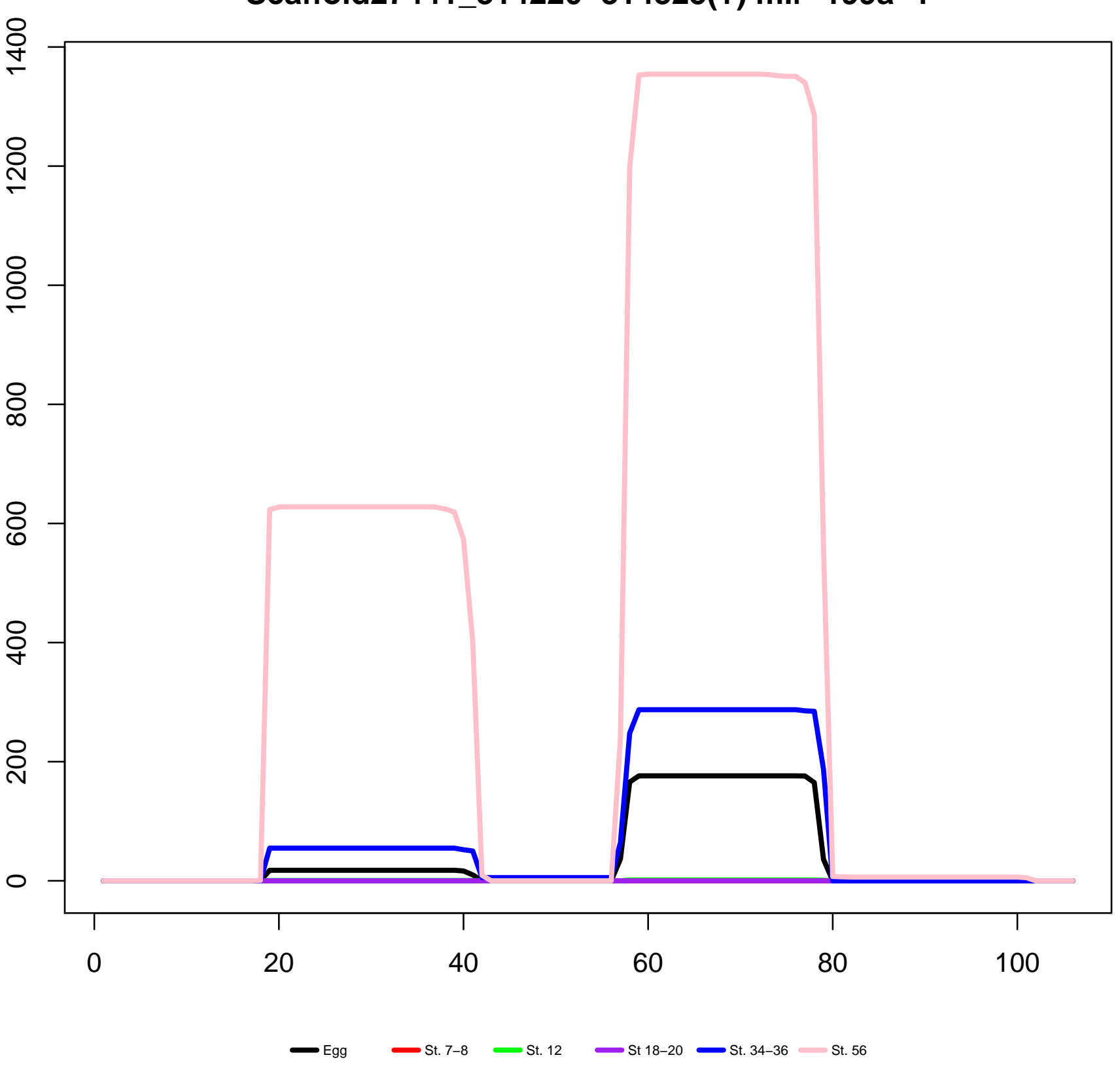


Scaffold27962_131742-131820(-) mir-24-2

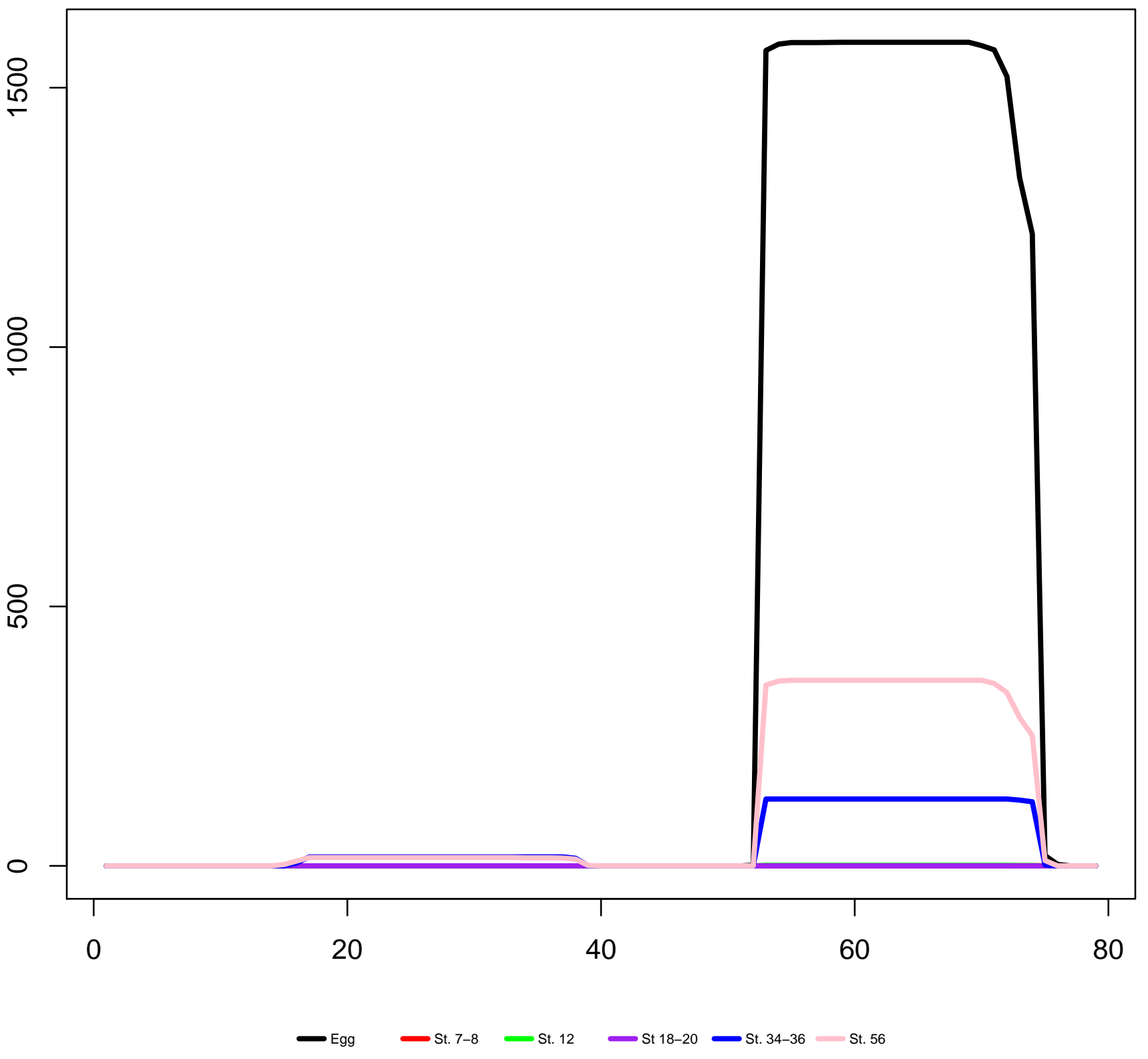


Scaffold28274_1083268-1083373(-) mir-10b

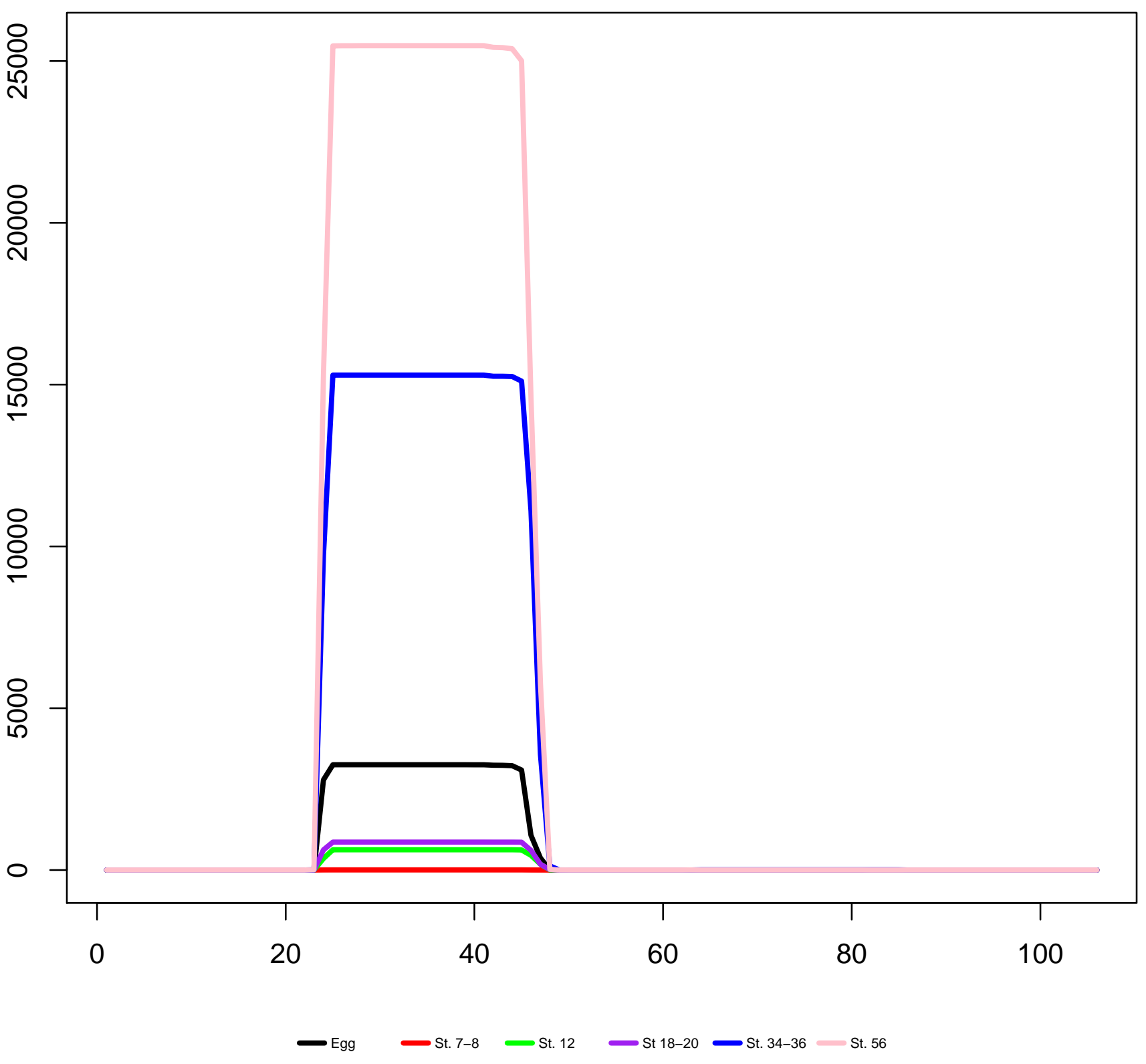


Scaffold28358_246515-246586(-) mir-16a

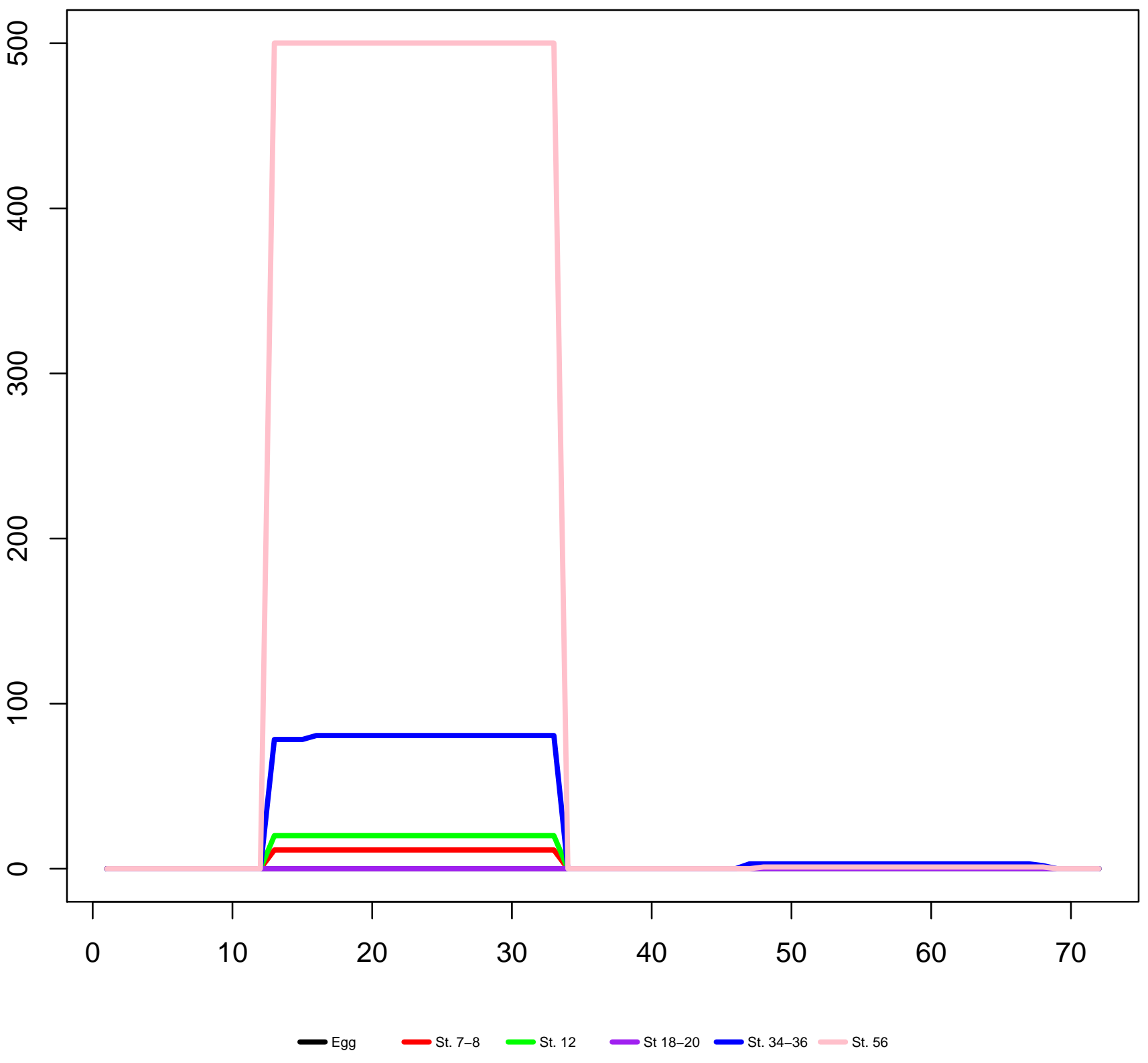


Scaffold28602_309438-309528(-) mir-101-2

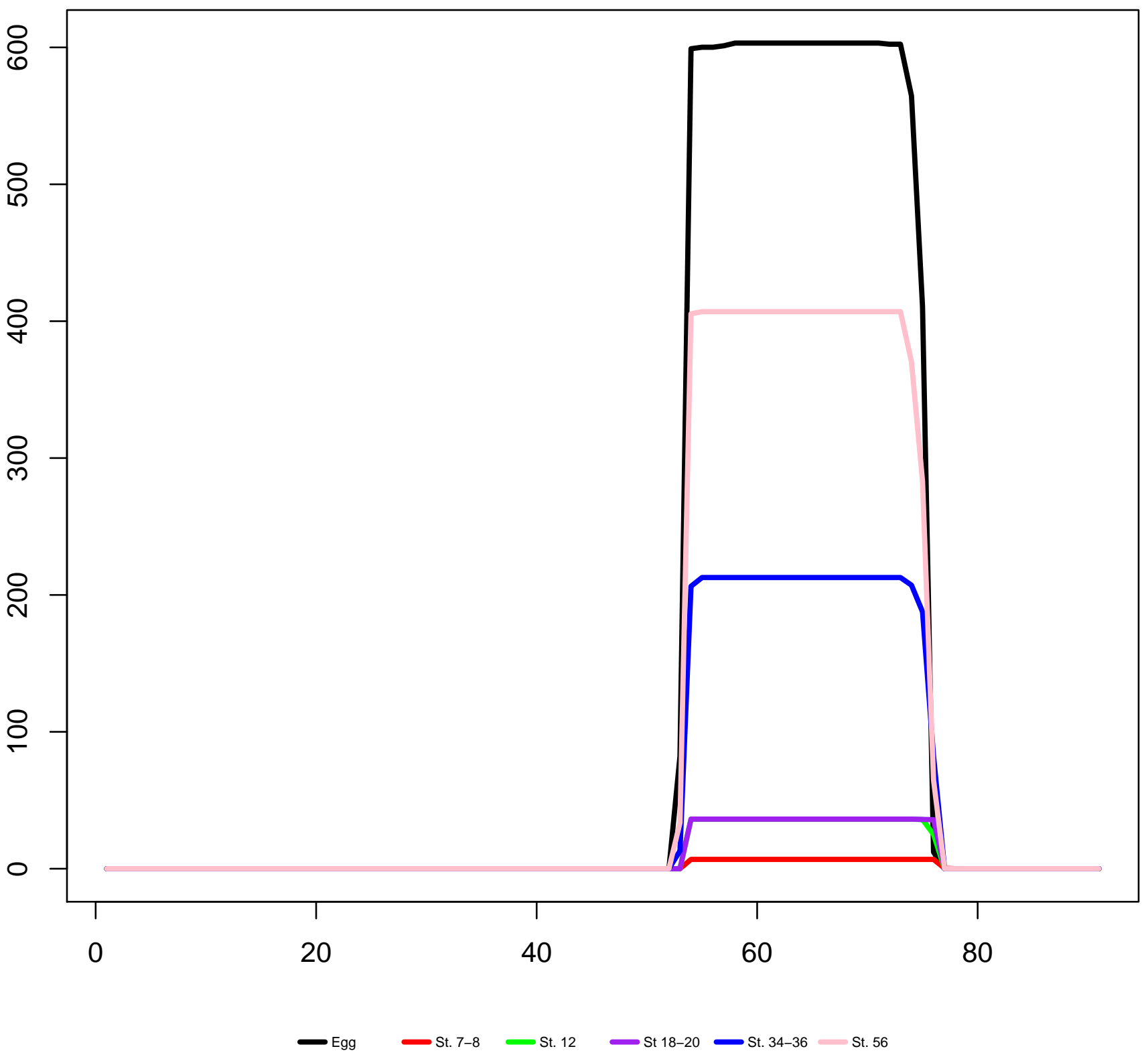


Scaffold28661_5269183-5269269(+) mir-202

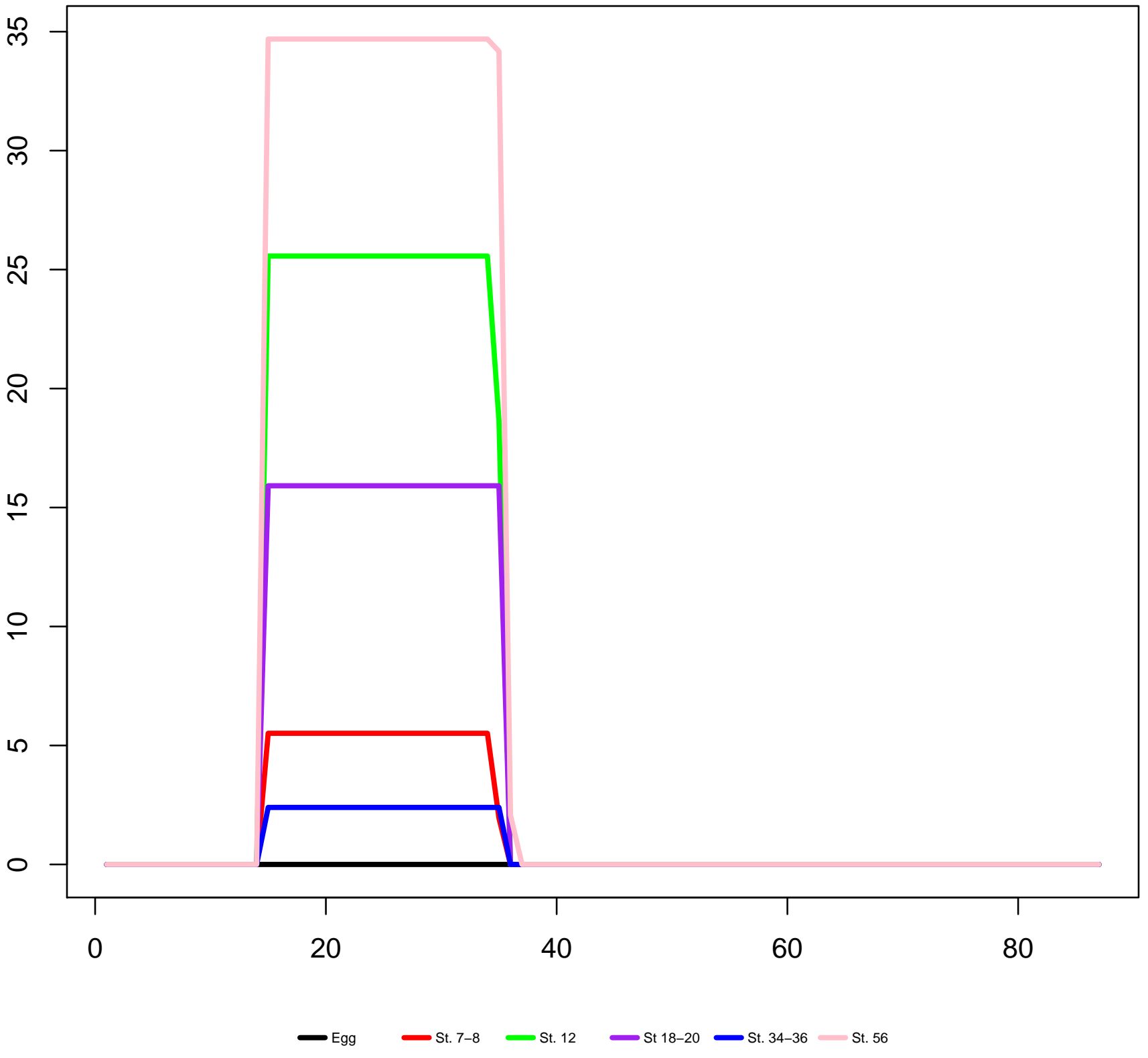


Scaffold28751_131567-131649(+) mir-124a-1

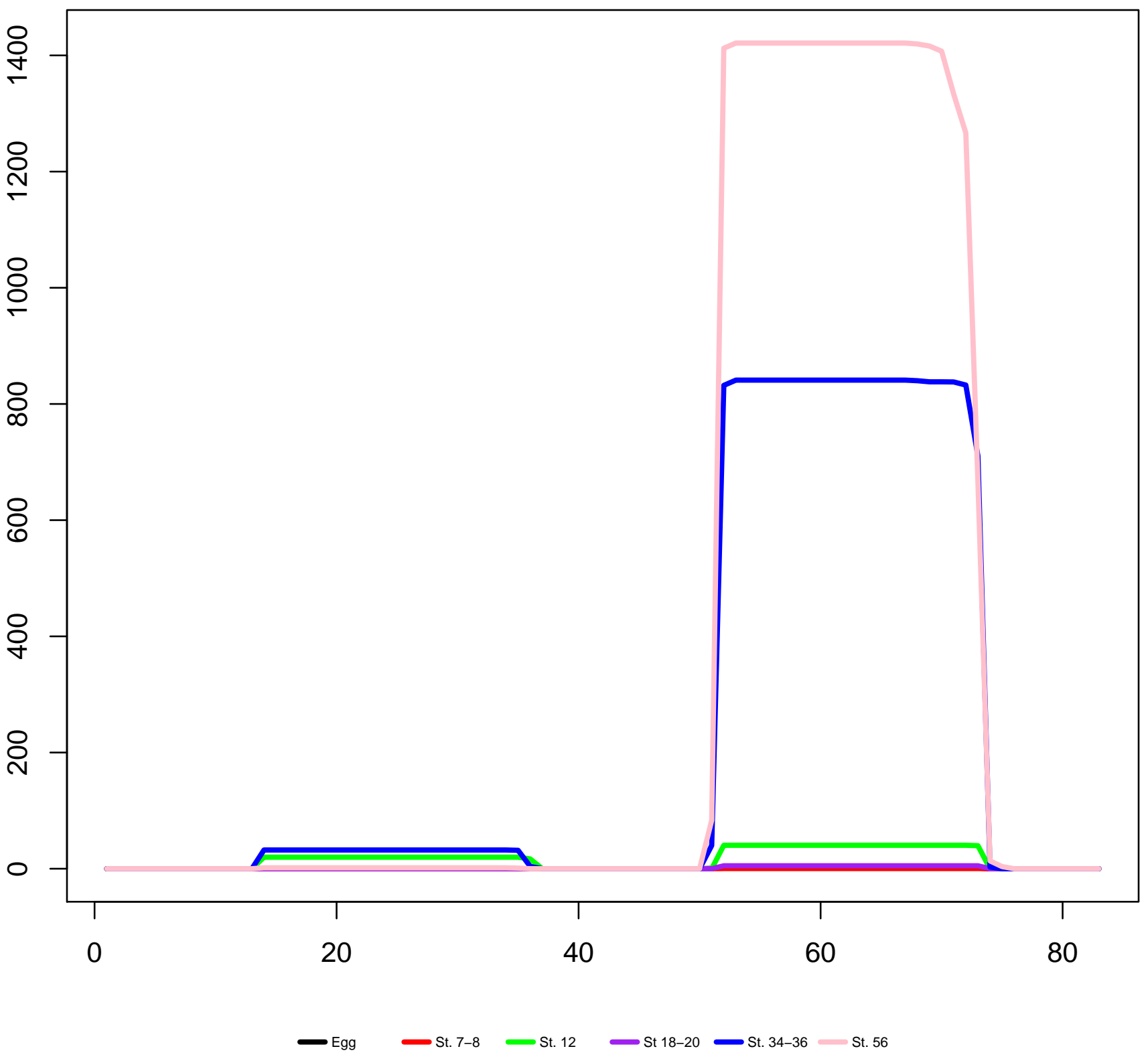


Scaffold28772_188732-188809(+) mir-122

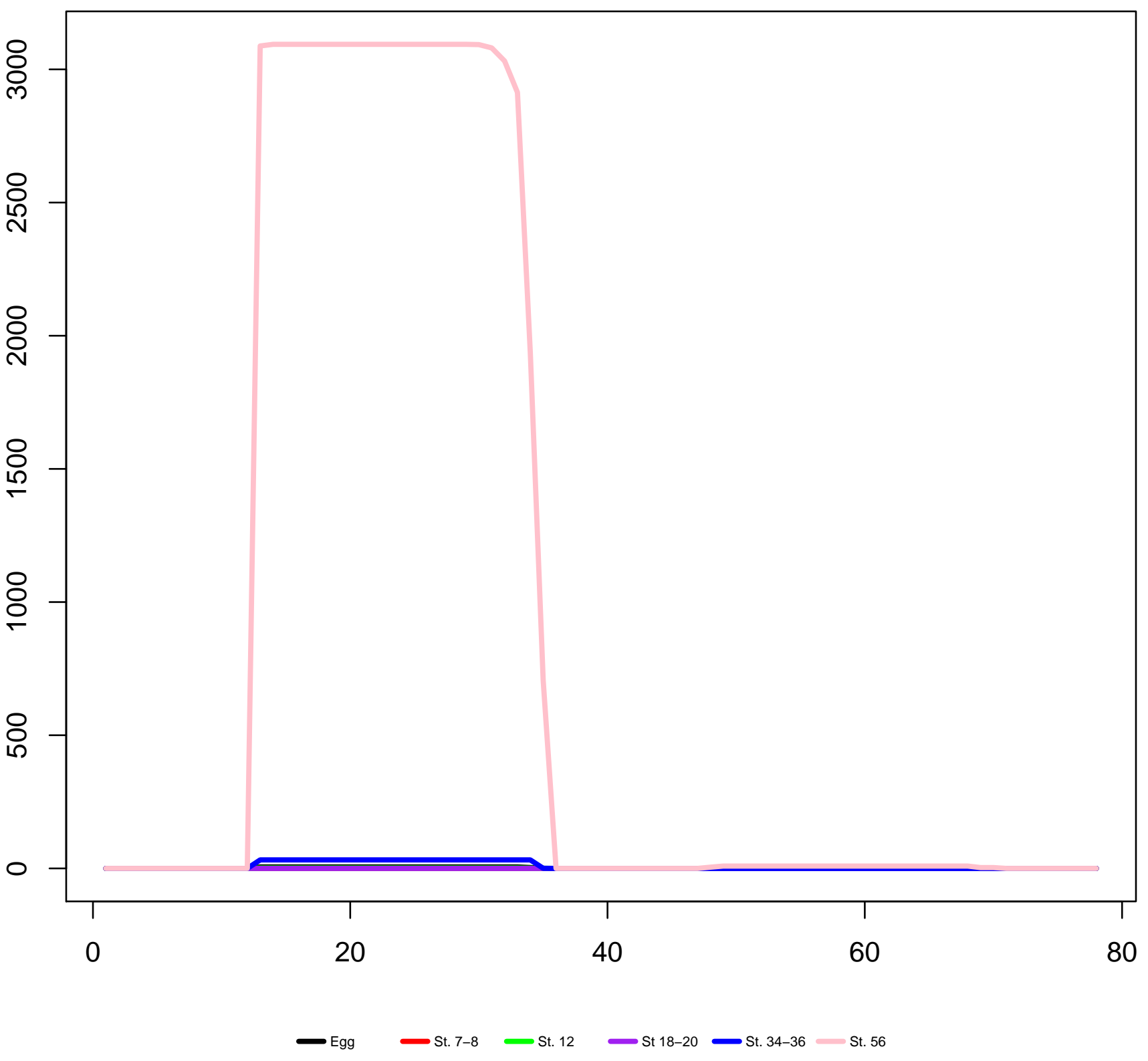


Scaffold29097_1251882-1252018(+) mir-135-2

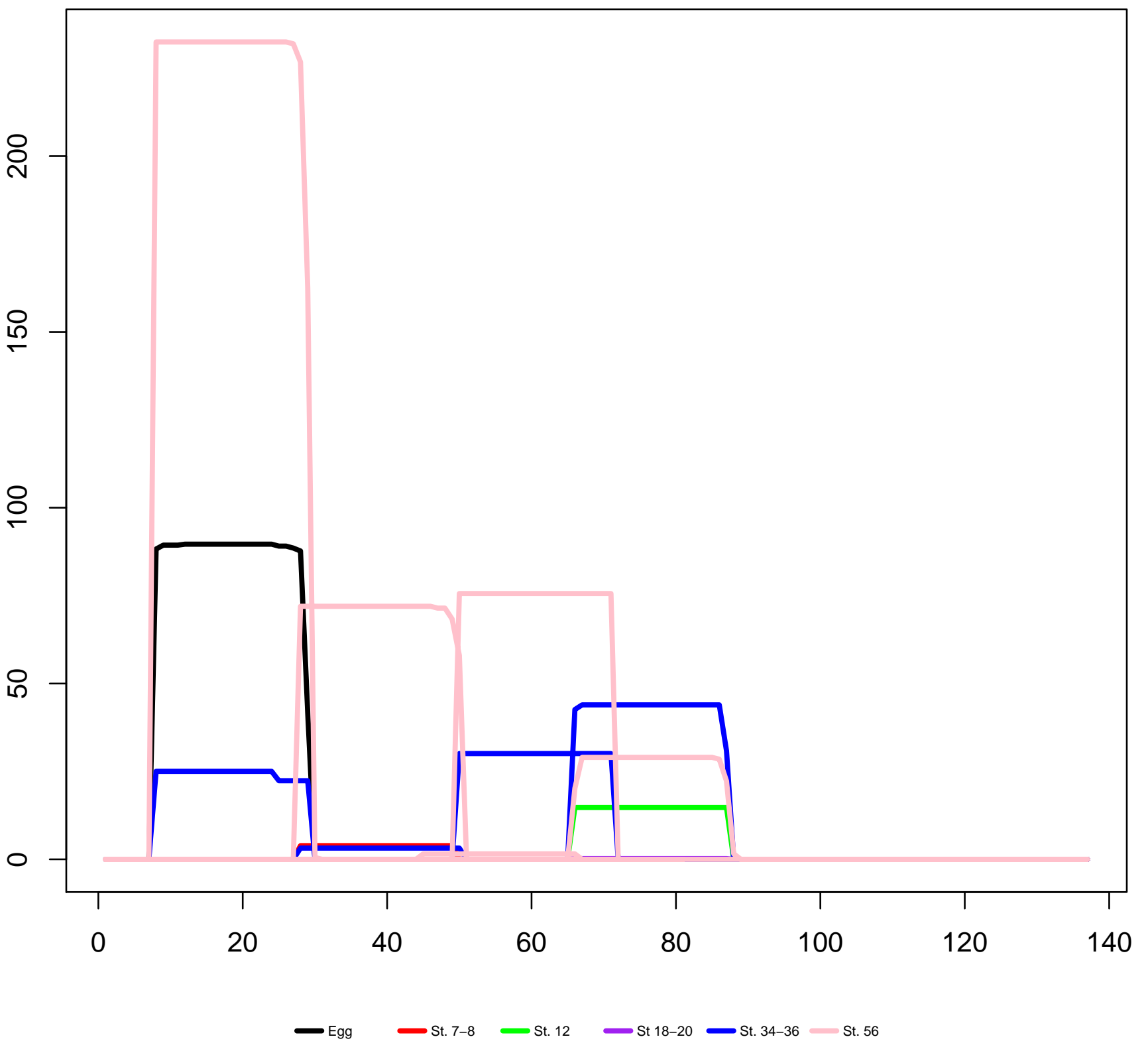


Scaffold3043_360261-360349(+) mir-17

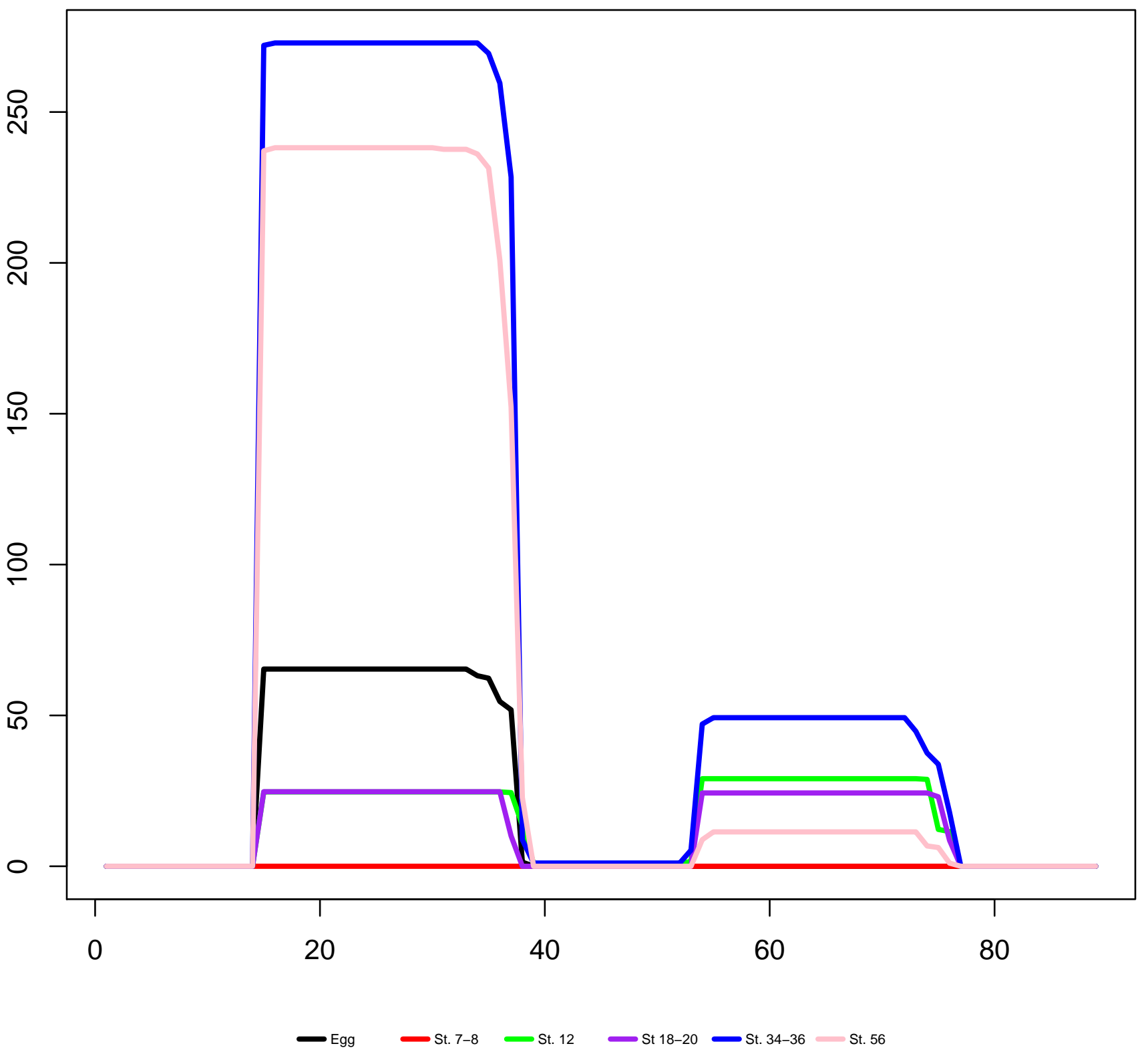


Scaffold3043_360392-360474(+) mir-18a

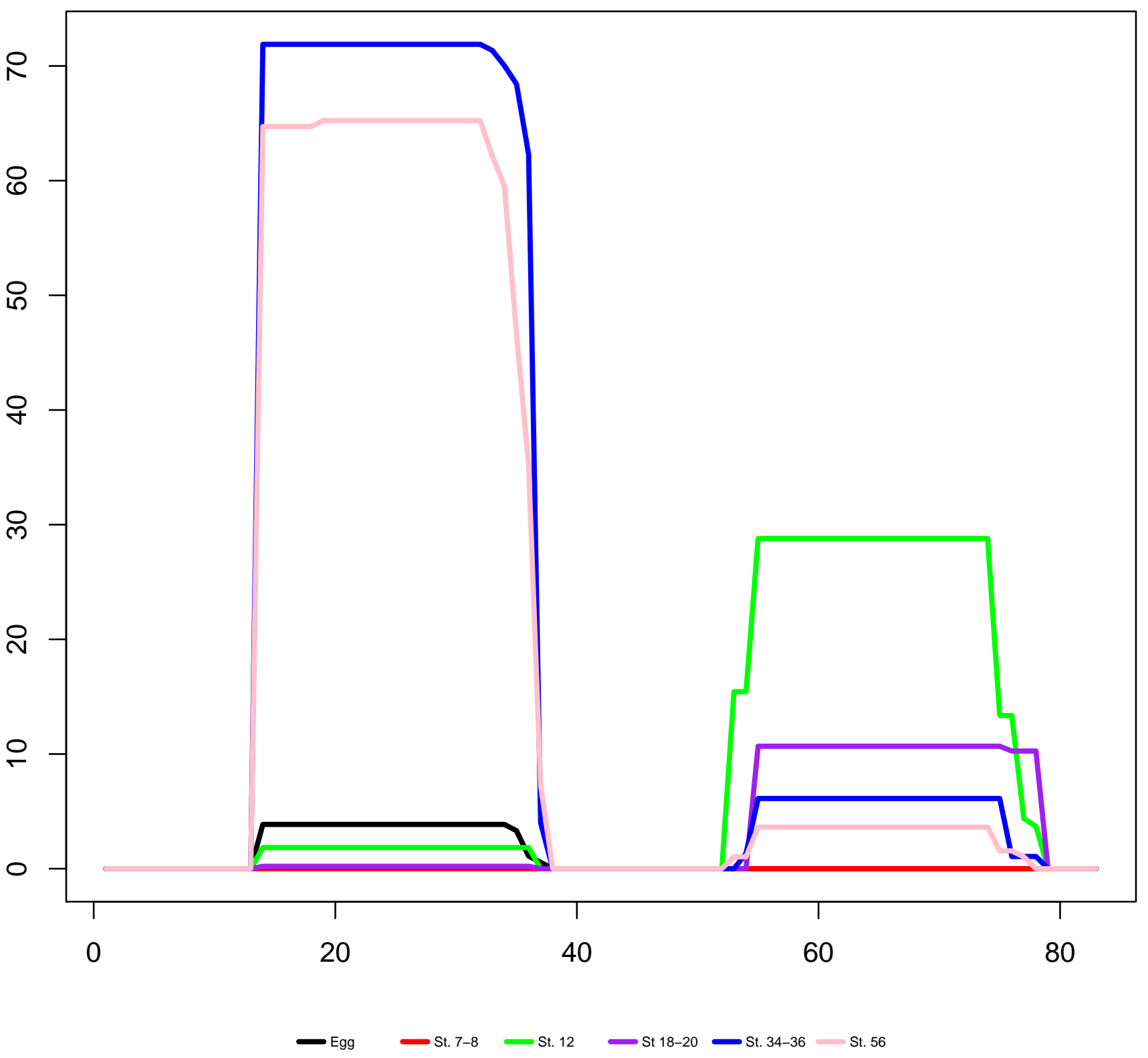


Scaffold3043_360530-360611(+) mir-19a

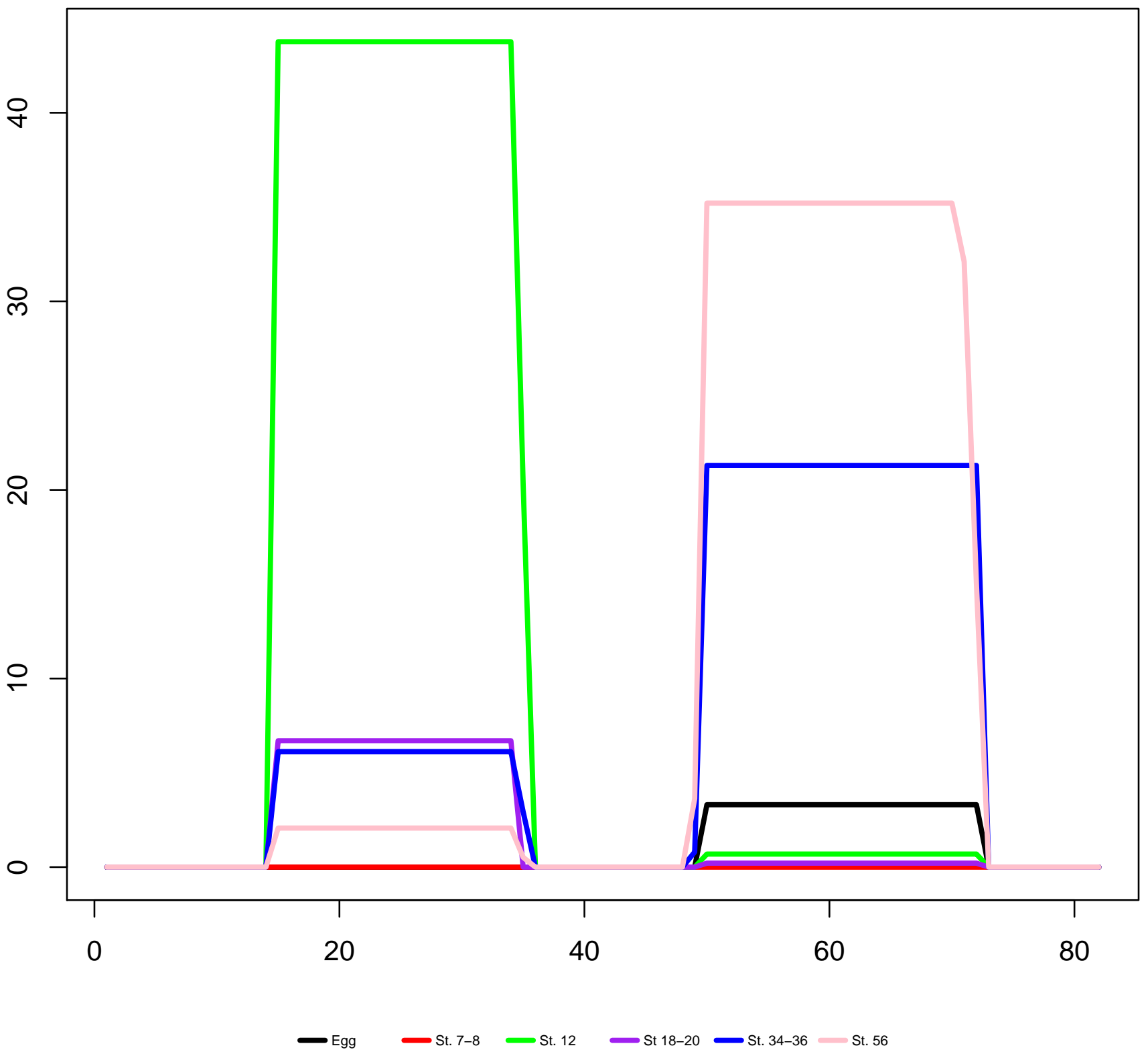


Scaffold3043_360827-360905(+) mir-19b

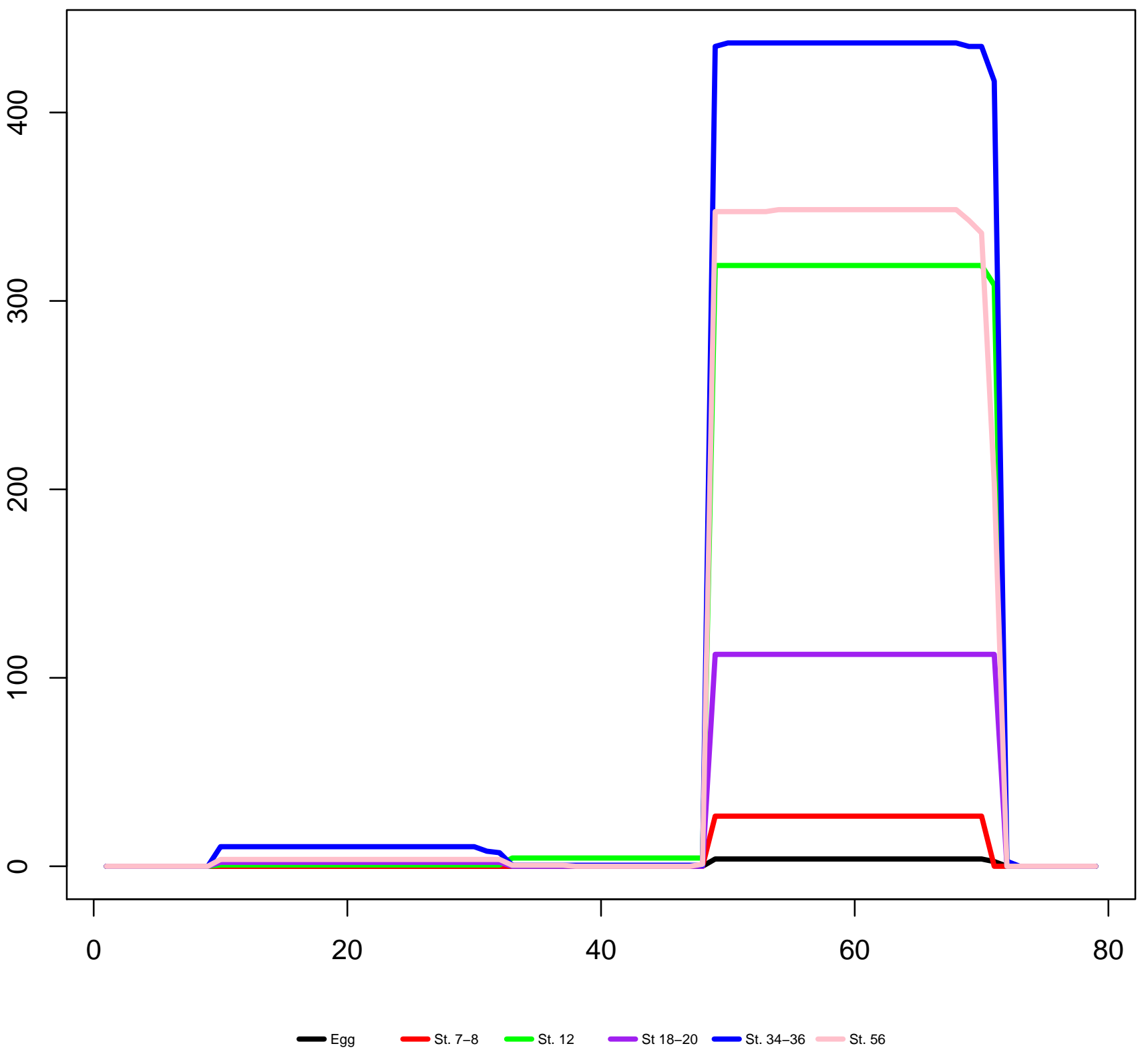


Scaffold3064_474145-474223(+) let-7a

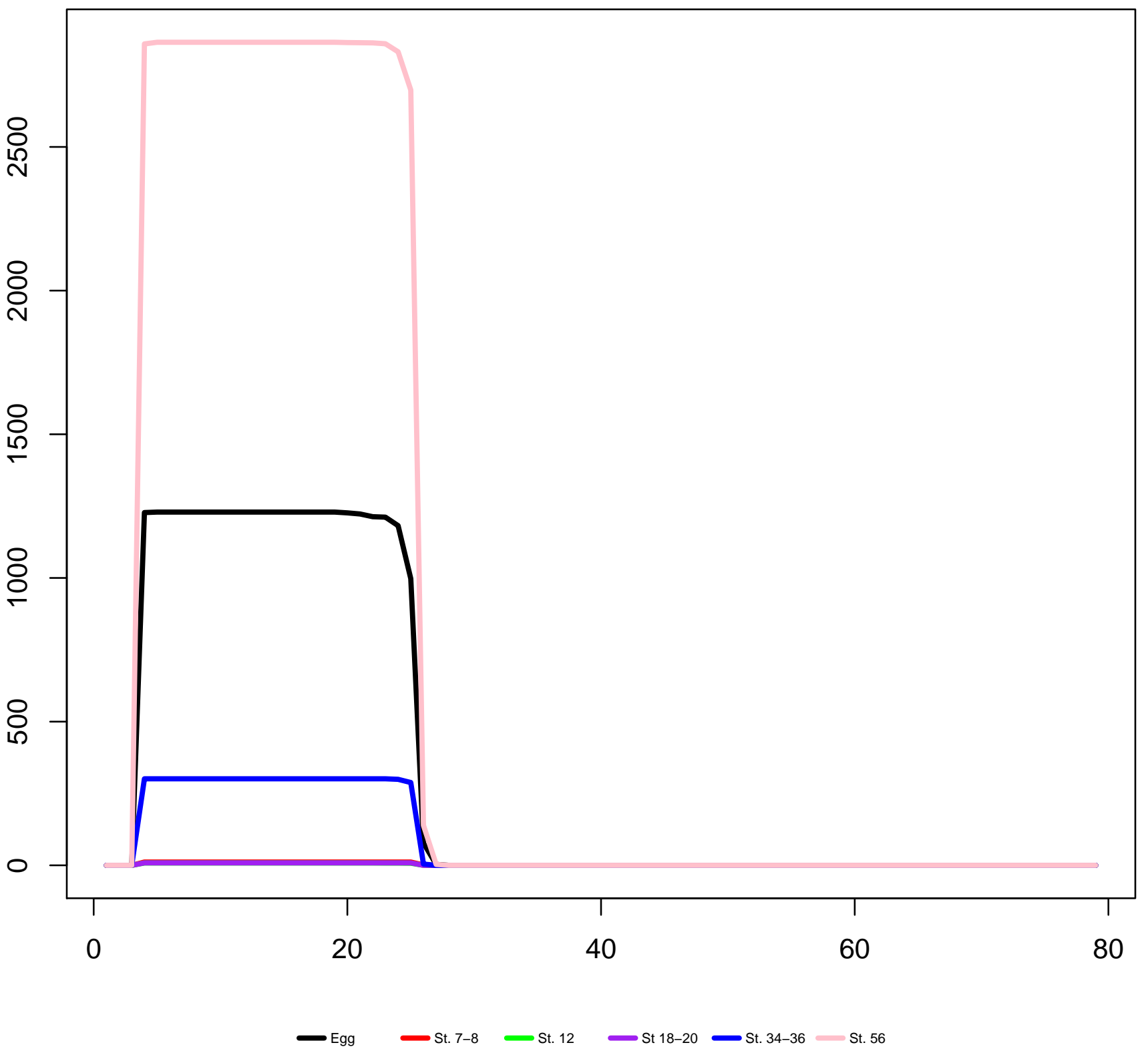


Scaffold30744_1672996-1673095(+) mir-153-1

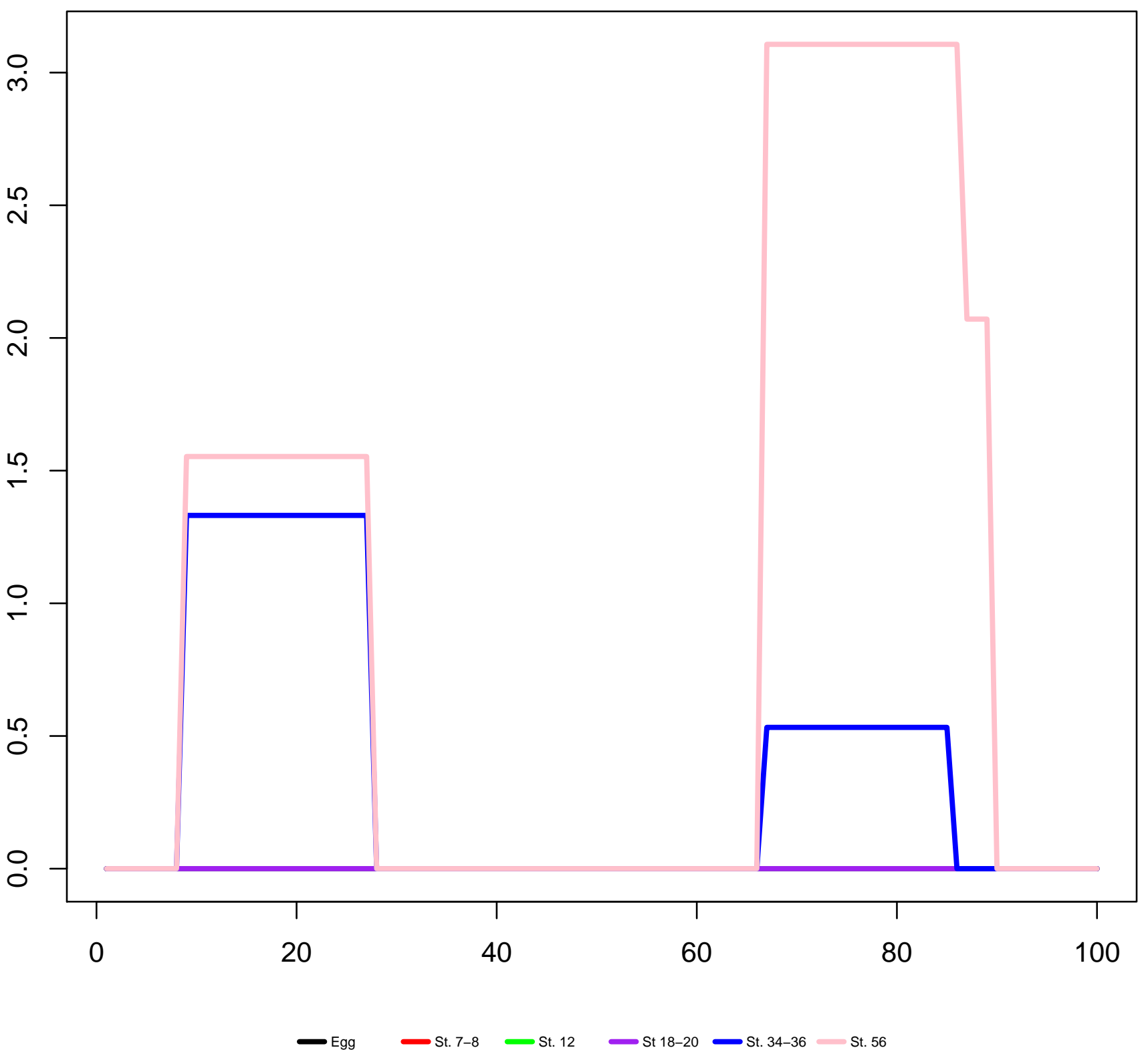


Scaffold31159_663264-663353(+) mir-181a-1

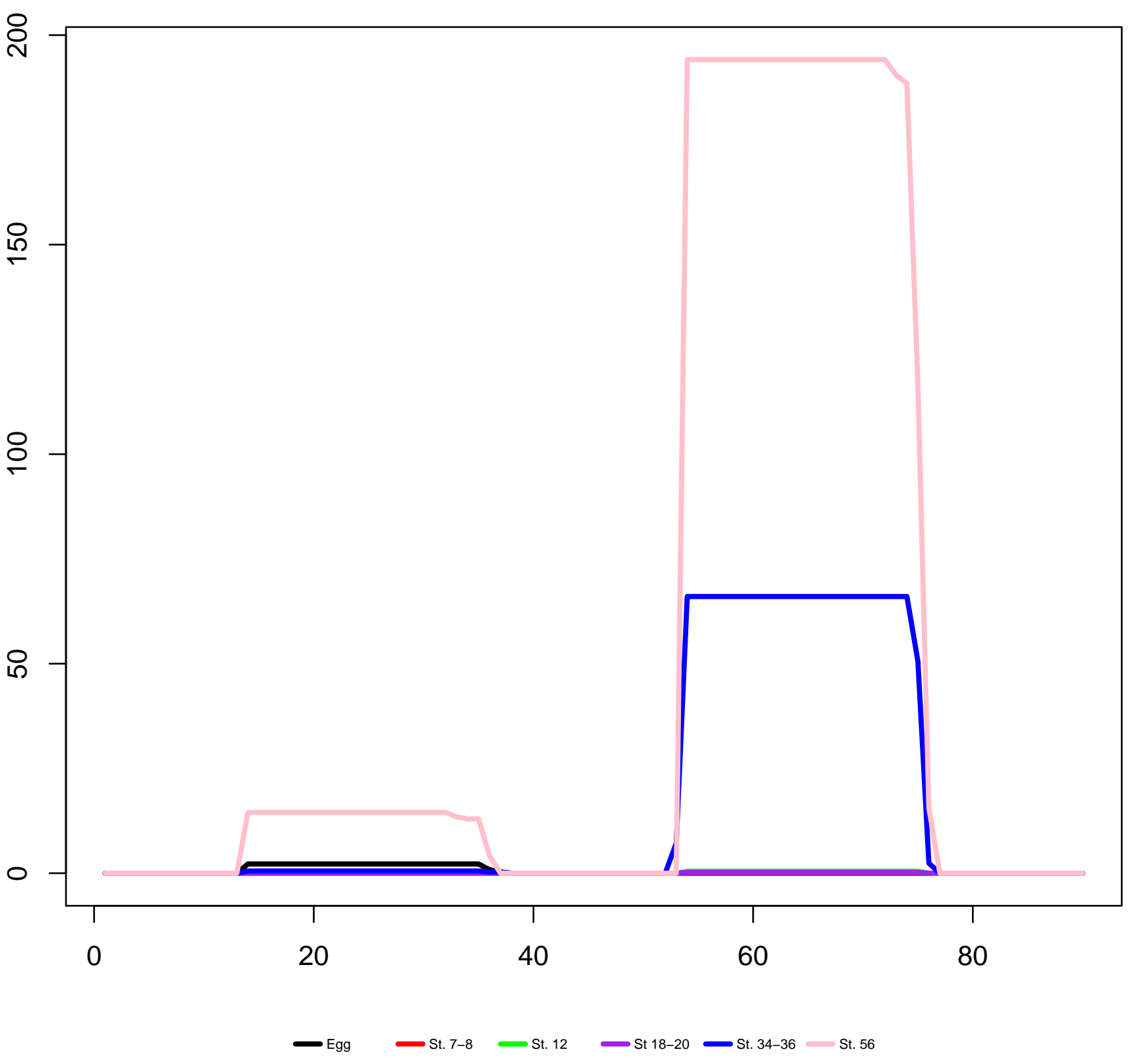


Scaffold31159_664074-664155(+) mir-181b-2

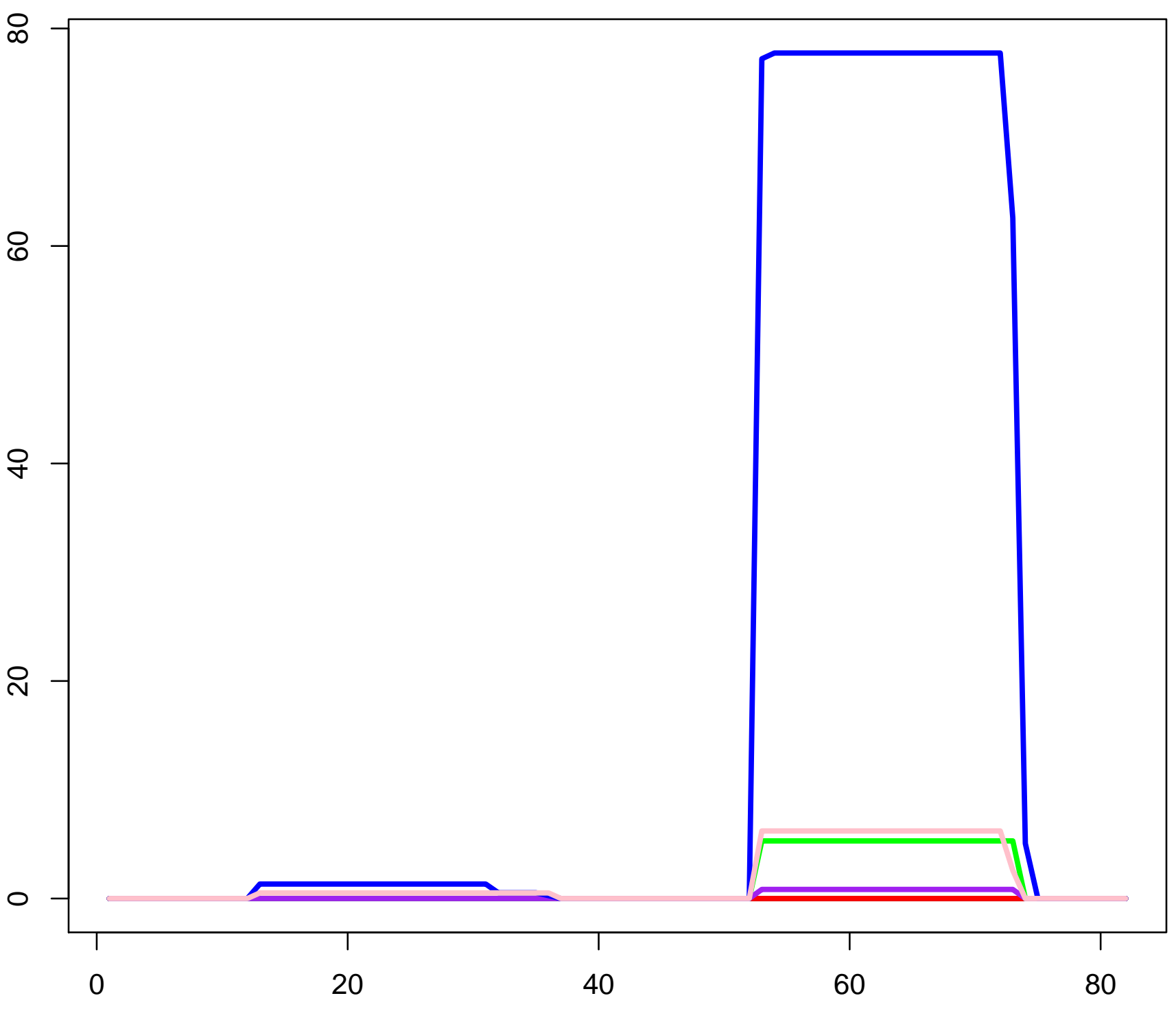

Egg $\rightleftharpoons$ St. 7-8 $\rightleftharpoons$ St. $12=$ St 18-20 $\rightleftharpoons$ St. 34-36 St. 56 
Scaffold31219_159145-159240(-) mir-146b

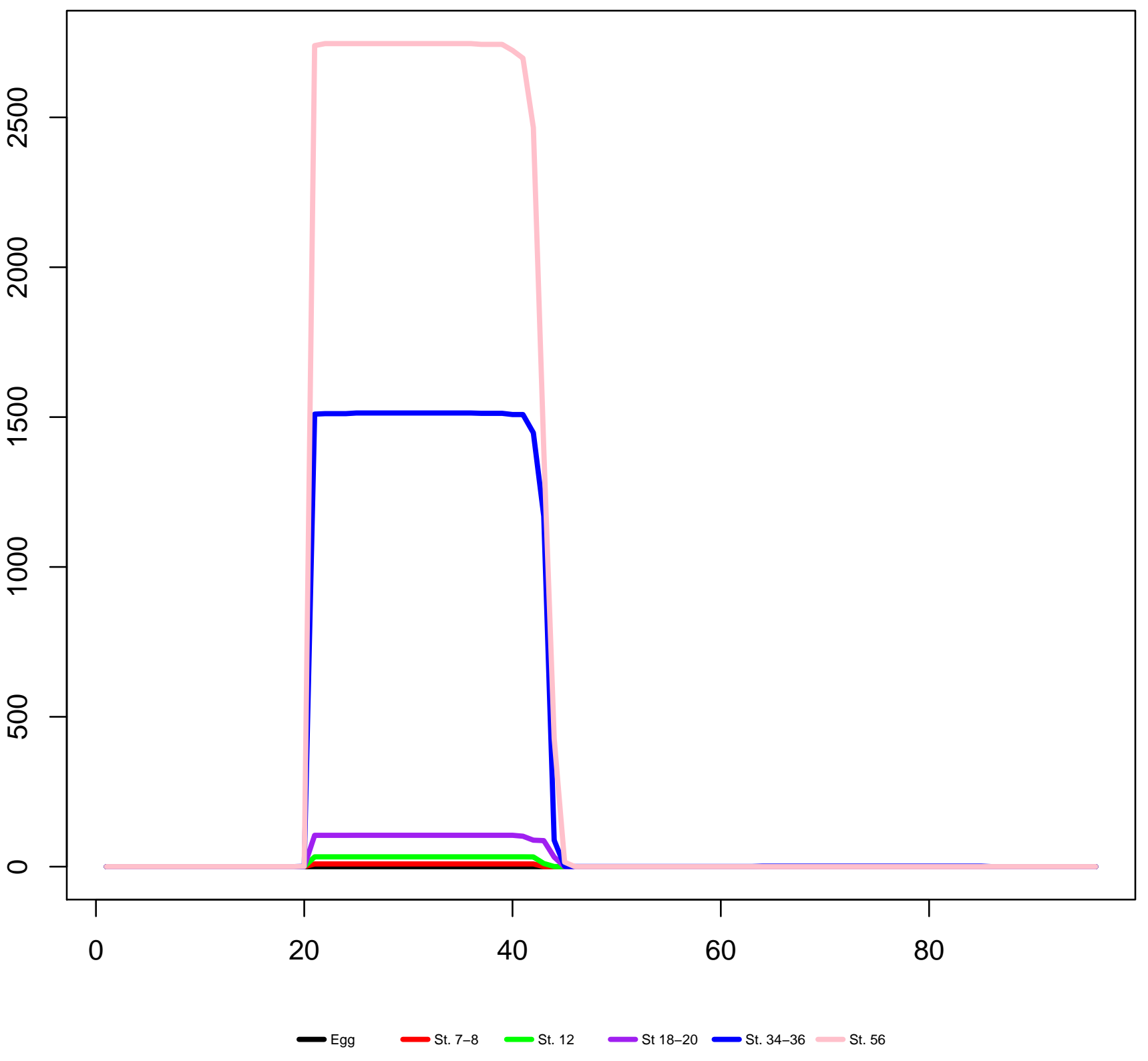




\section{Scaffold313941_15-100(-) mir-3553}

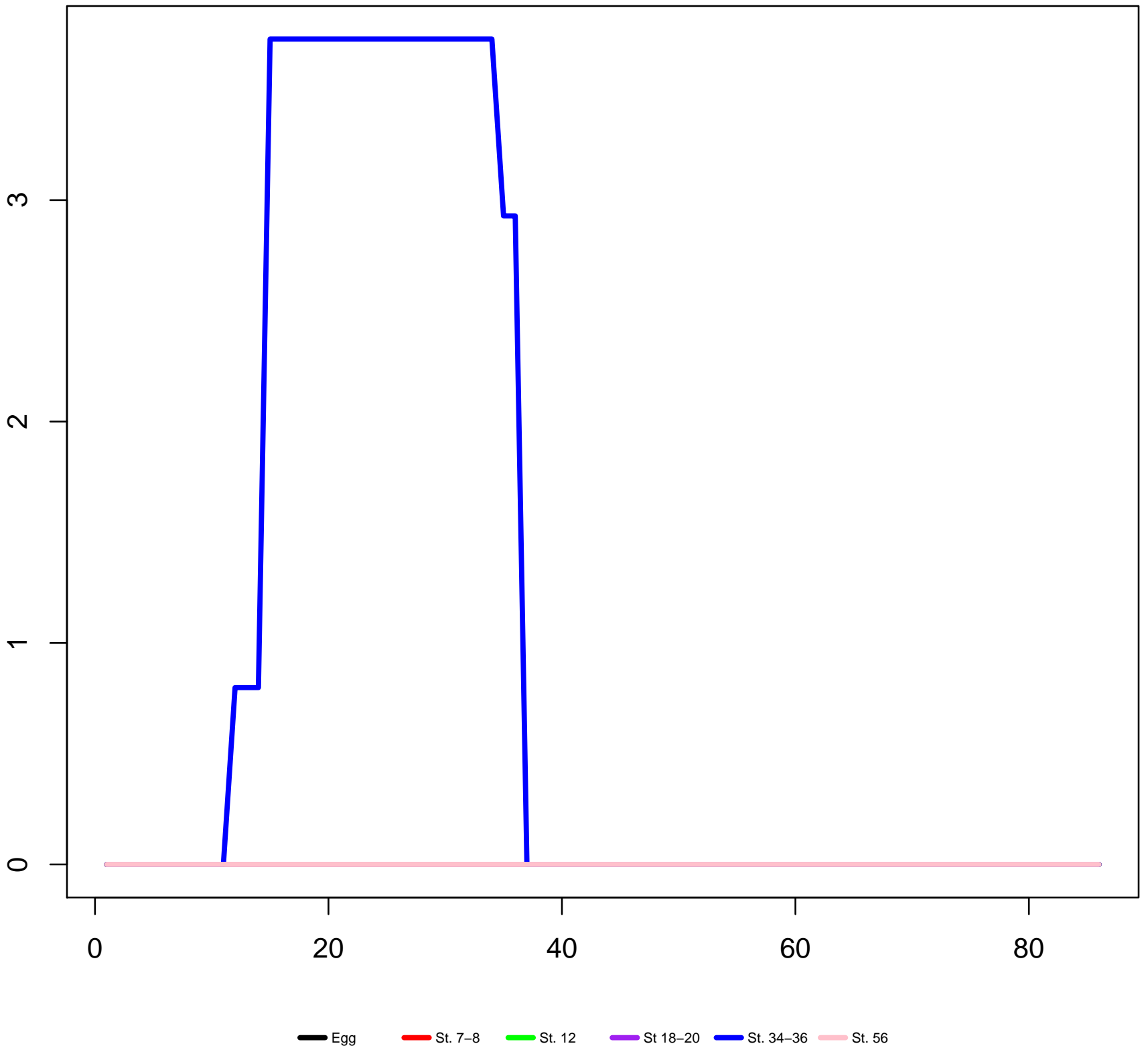


Scaffold31520_348179-348260(-) mir-31

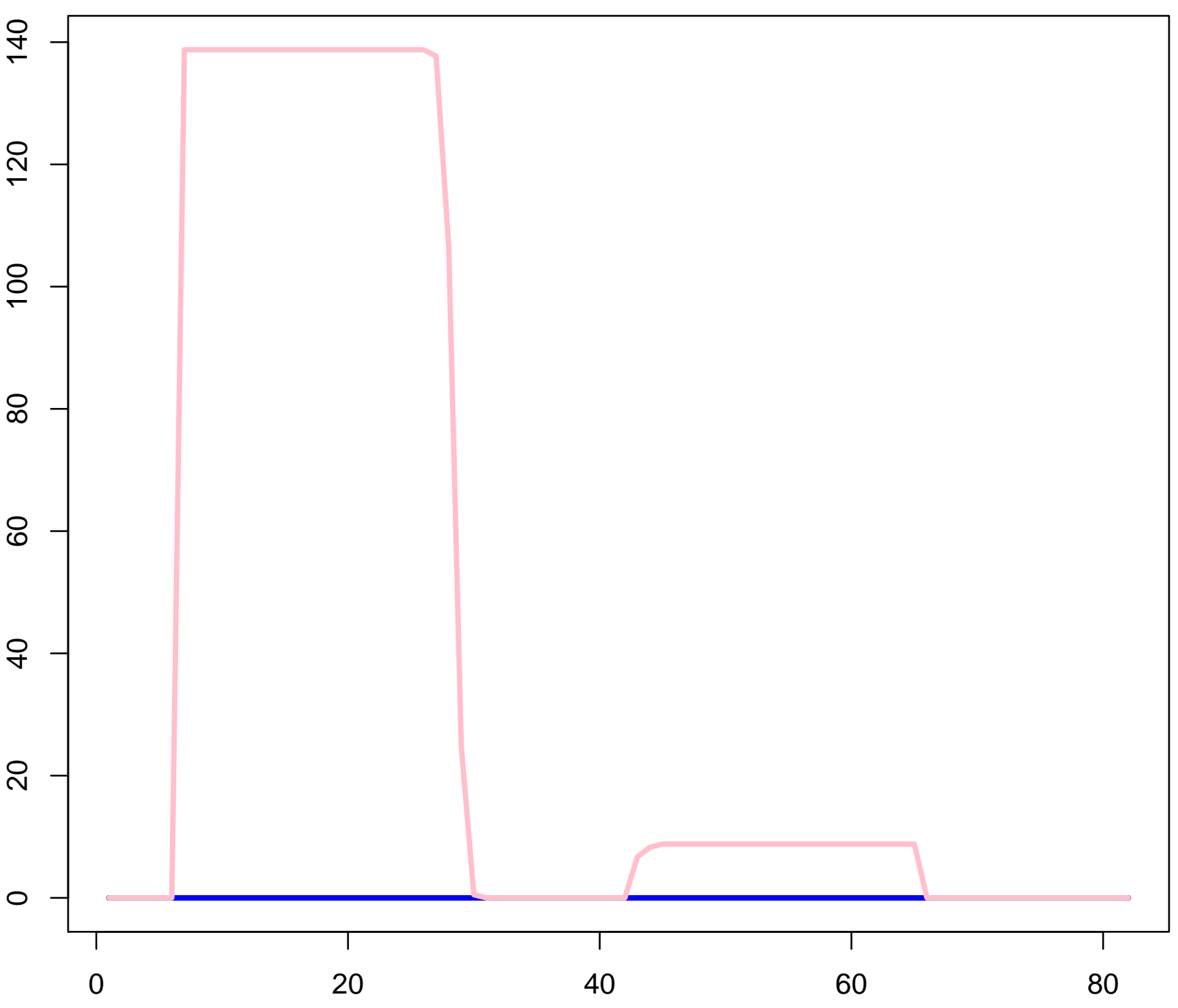


Scaffold32152_446292-446367(-) mir-138-2

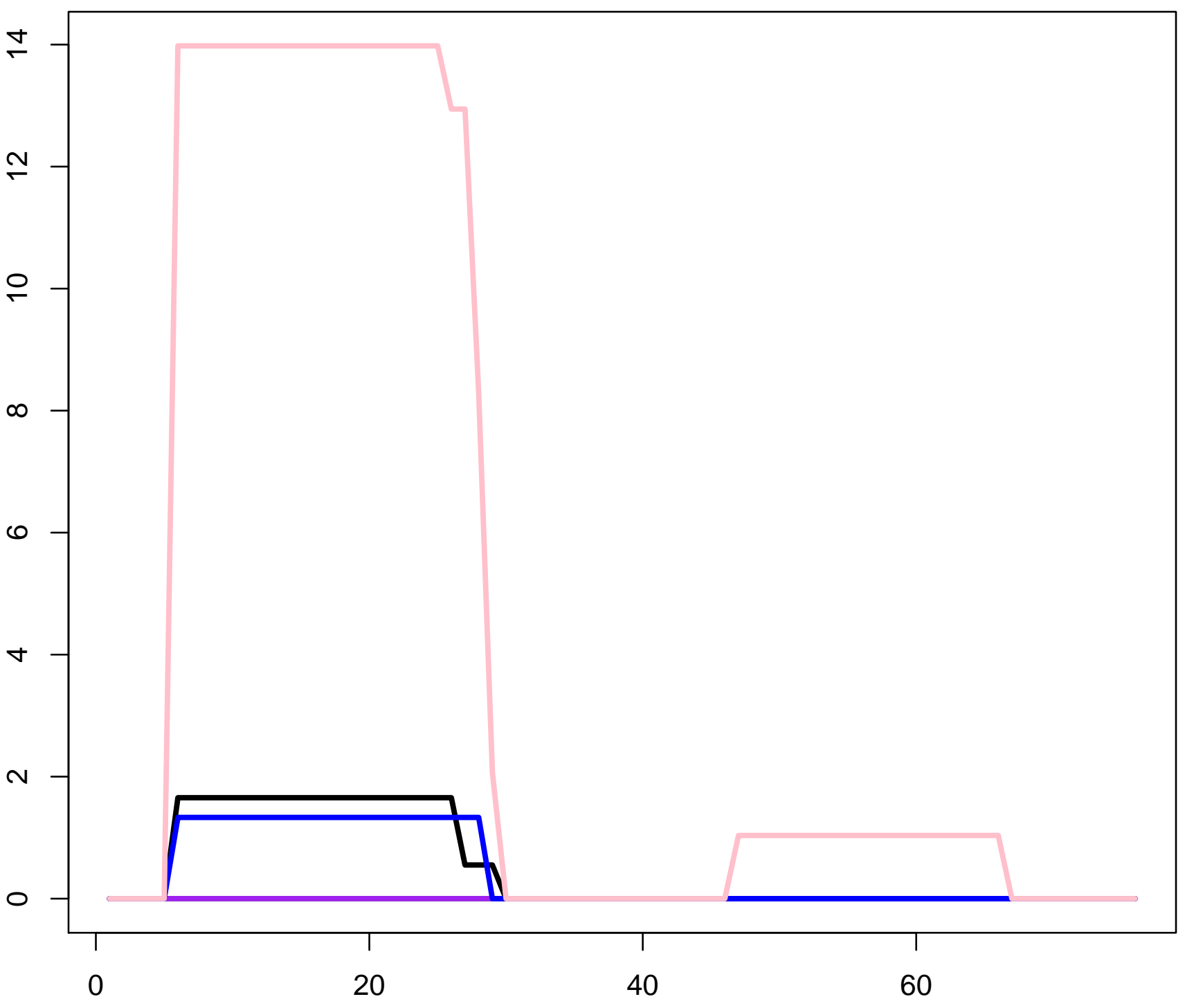


Scaffold32156_53194-53295(+) mir-10b

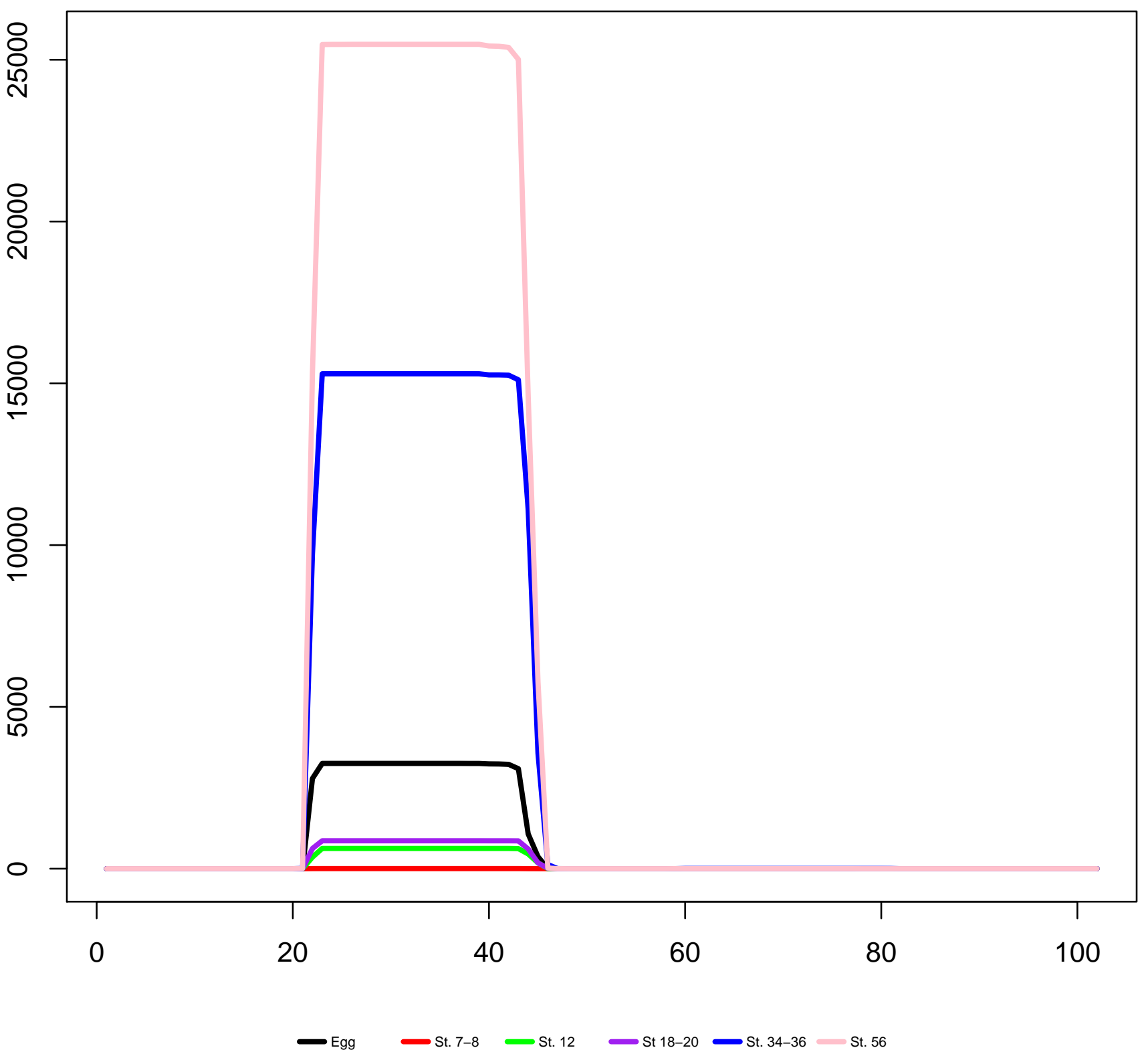


Scaffold32240_167532-167609(-) mir-9-3

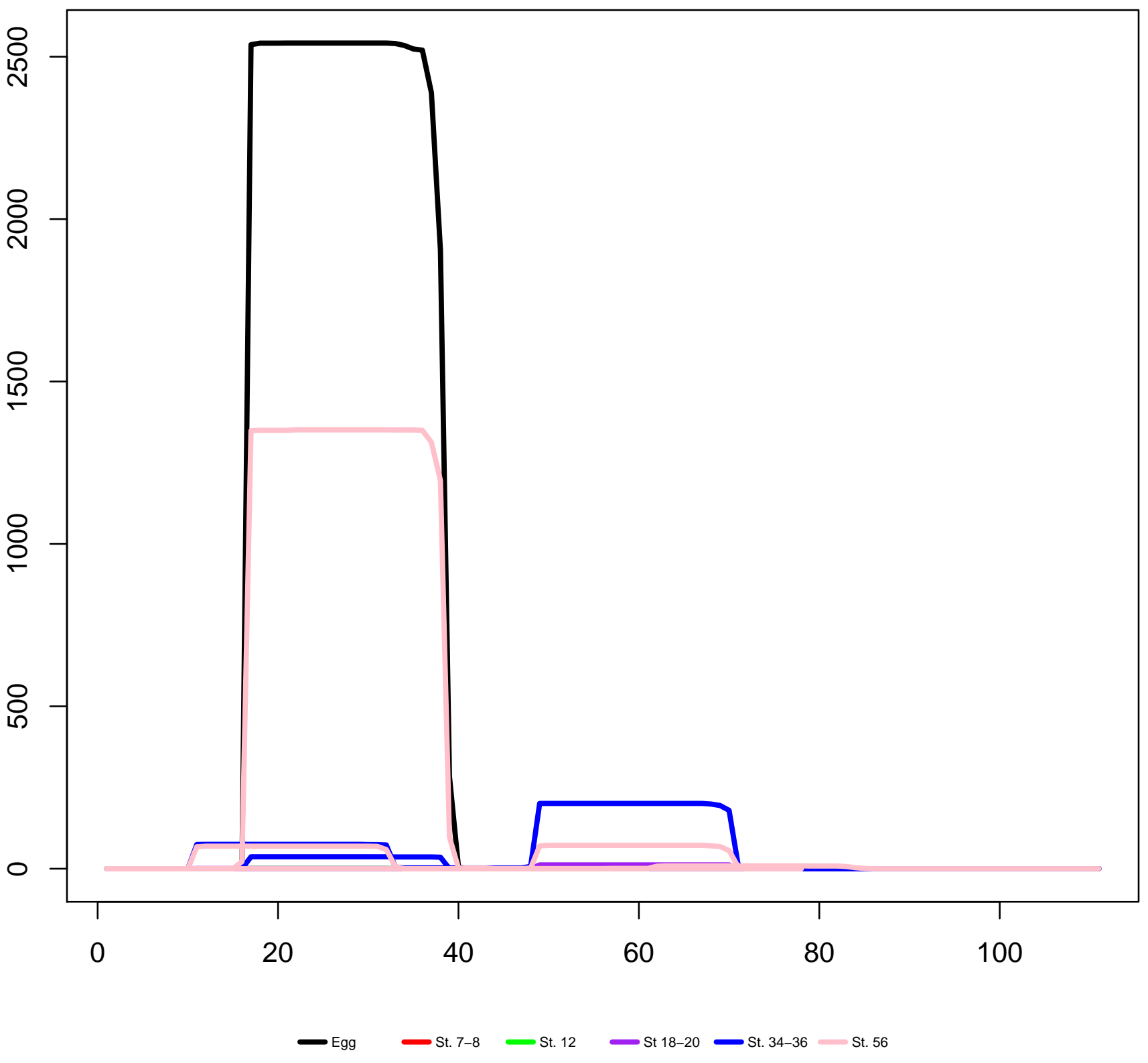


Scaffold3254_770098-770213(+) mir-196a-2

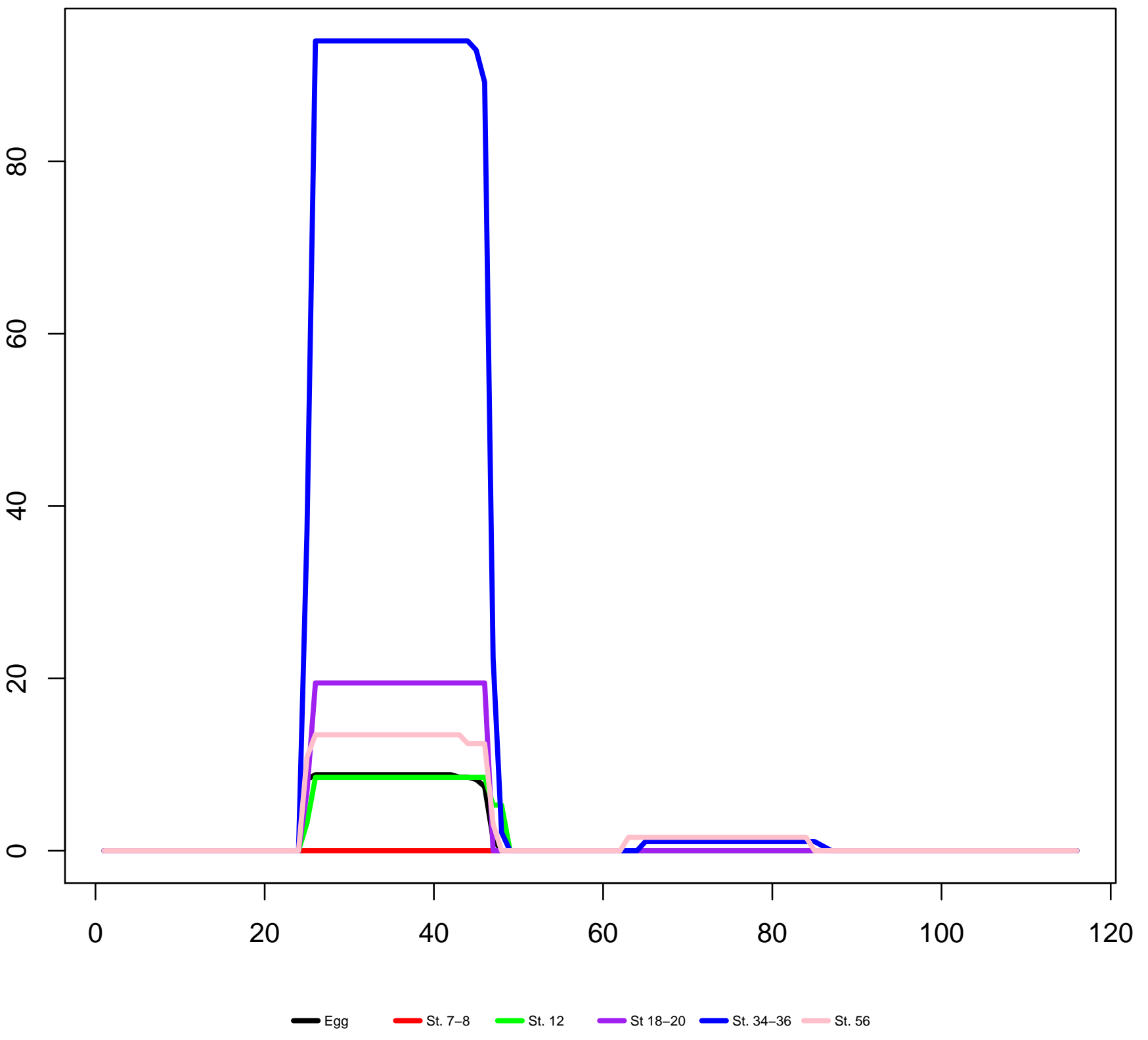


Scaffold3254_865362-865433(+) mir-10c

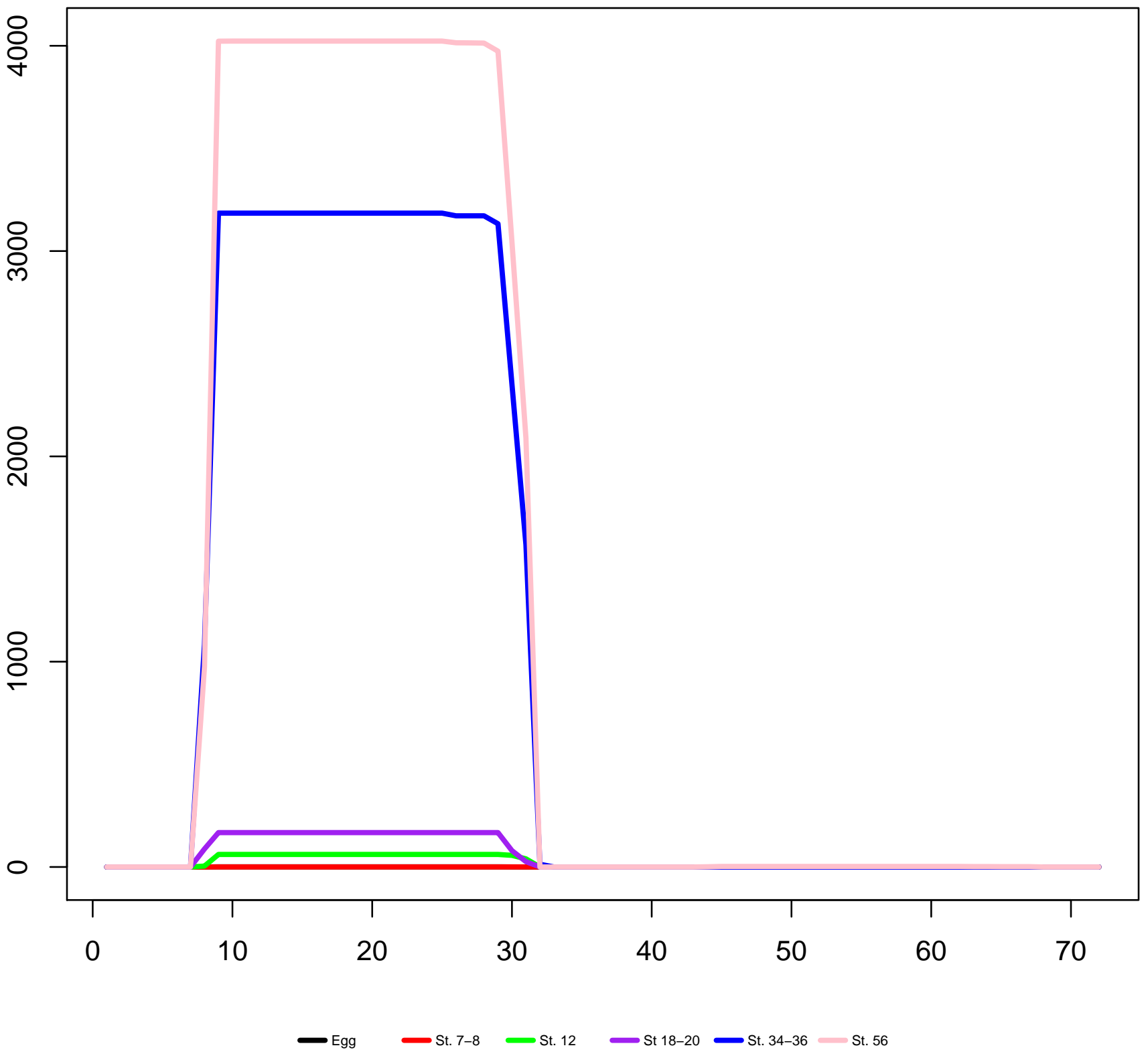




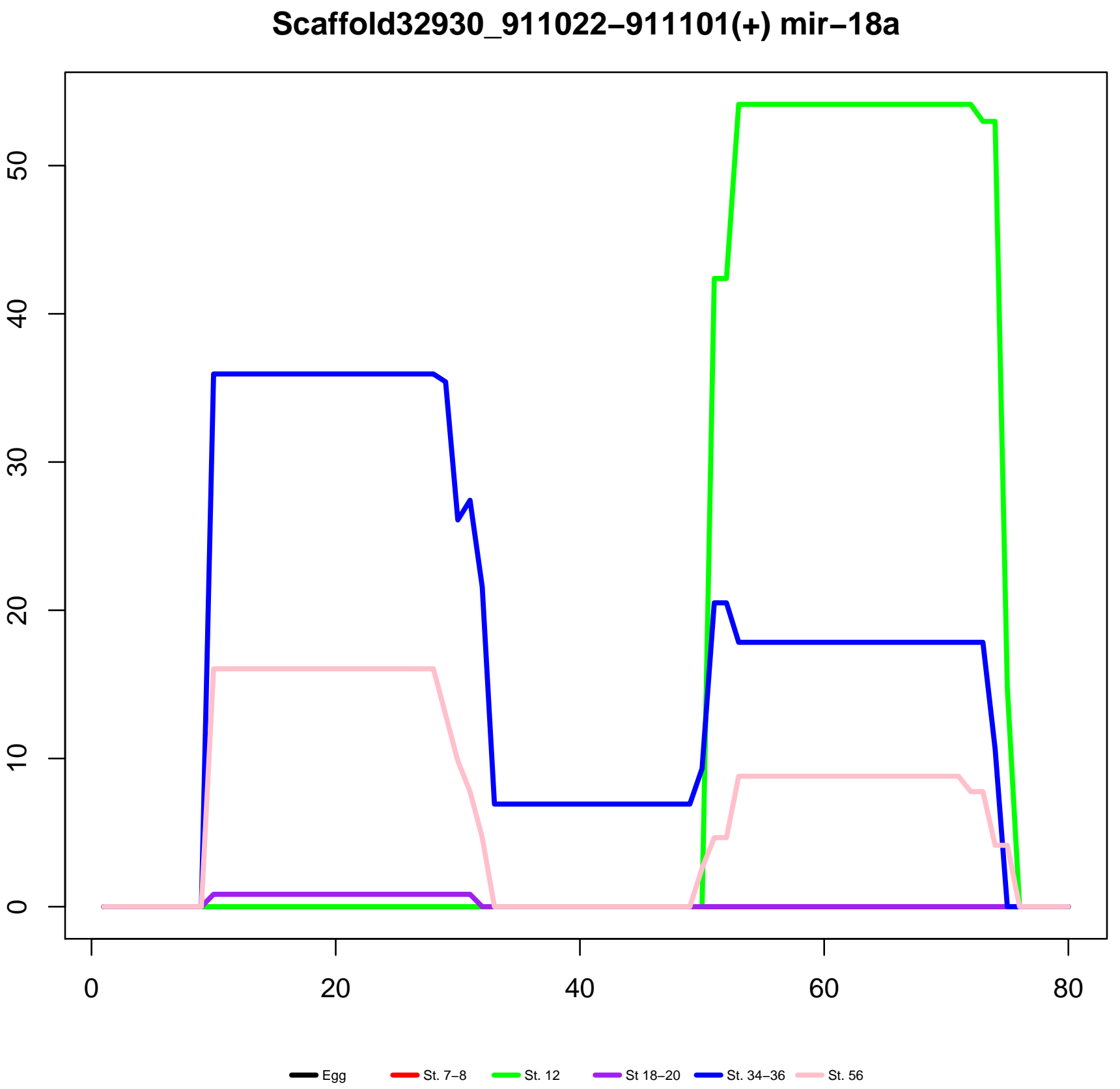


Scaffold32930_911178-911251(+) mir-20

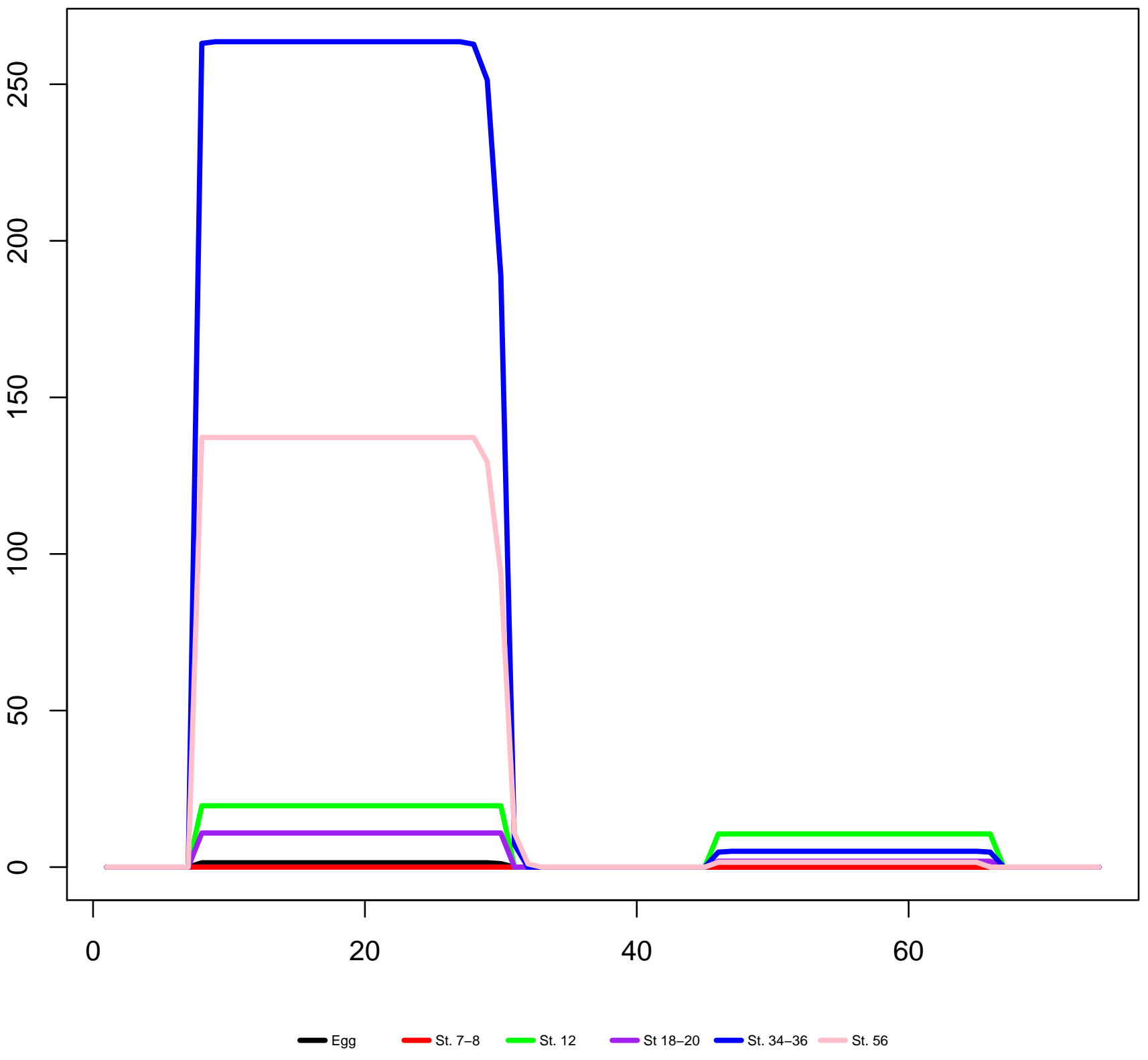


Scaffold32930_911289-911361(+) mir-19b

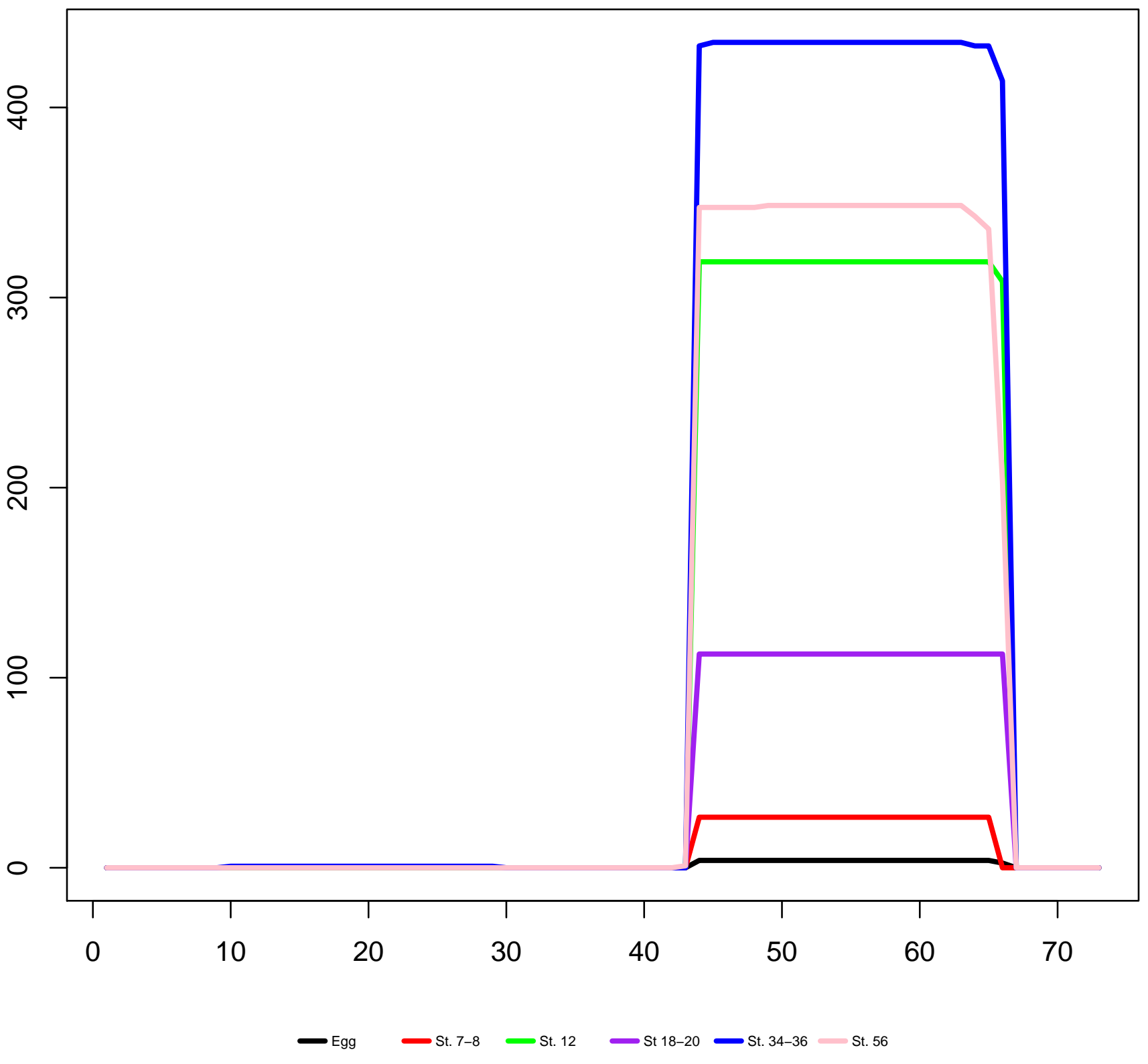


Scaffold3322_1136881-1136952(+) mir-26-2

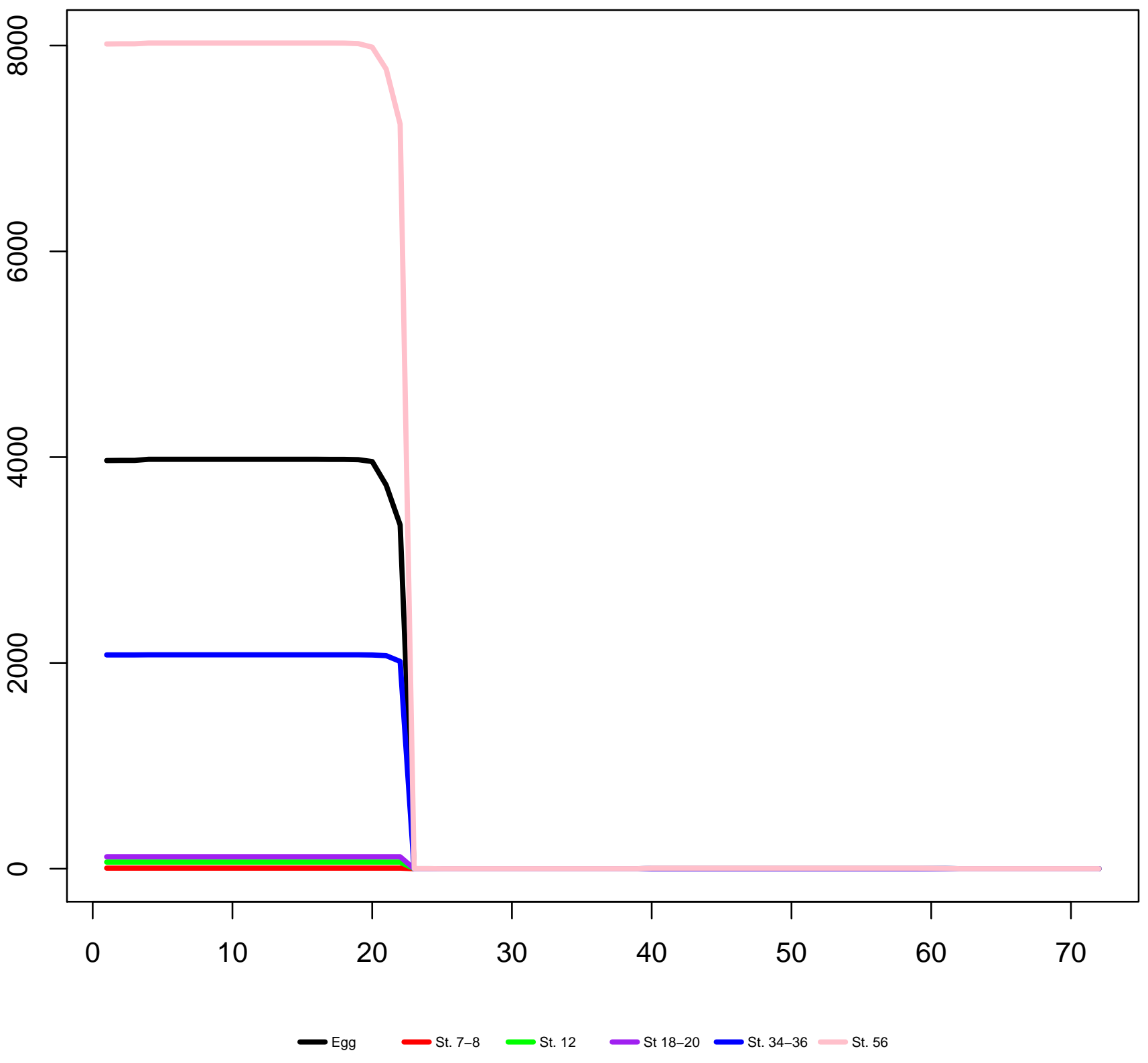


Scaffold3334_41555-41635(-) mir-128-1

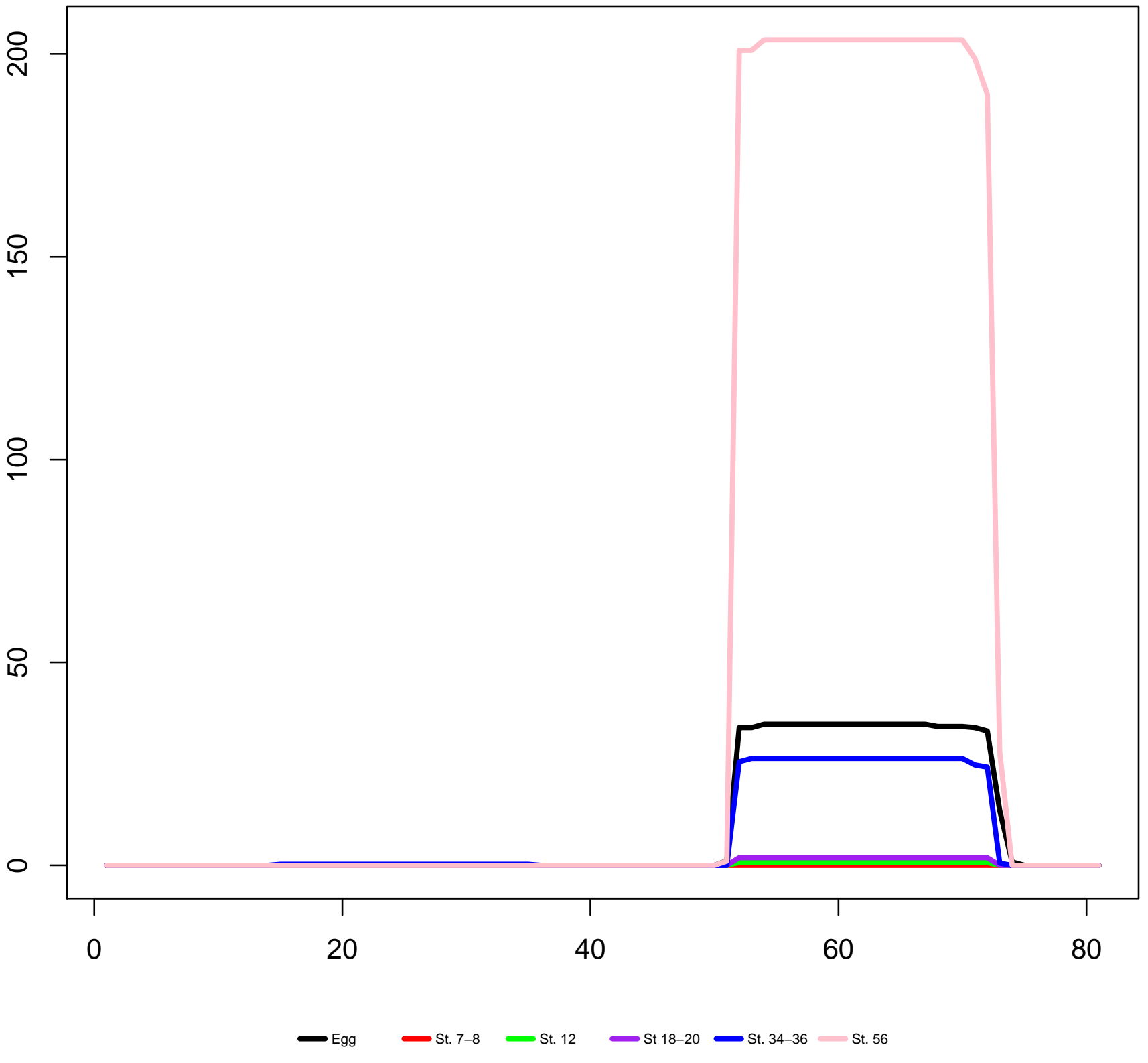


Scaffold33937_1493727-1493814(-) mir-181b-2

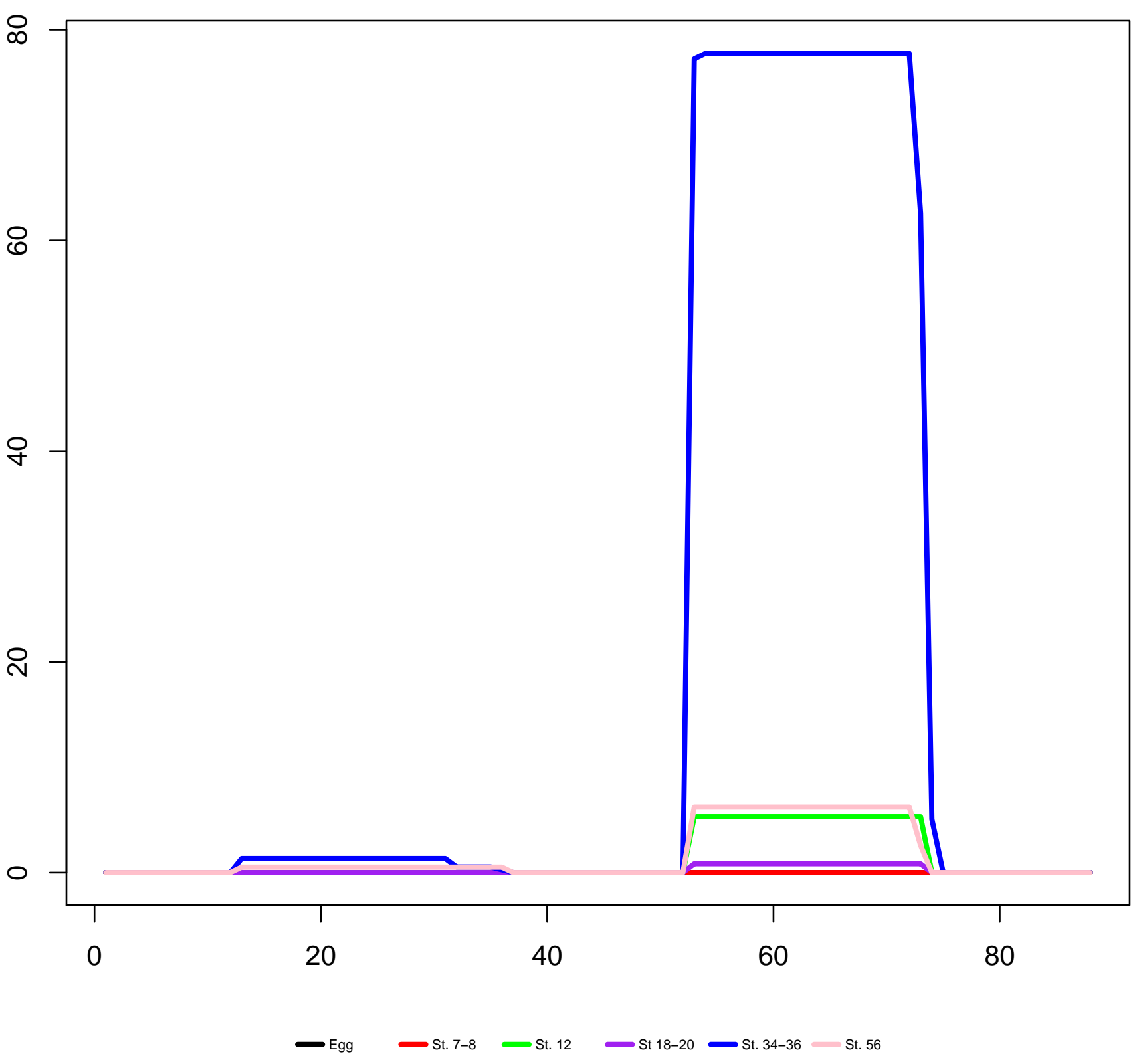


Scaffold33937_1494806-1494895(-) mir-181a-2

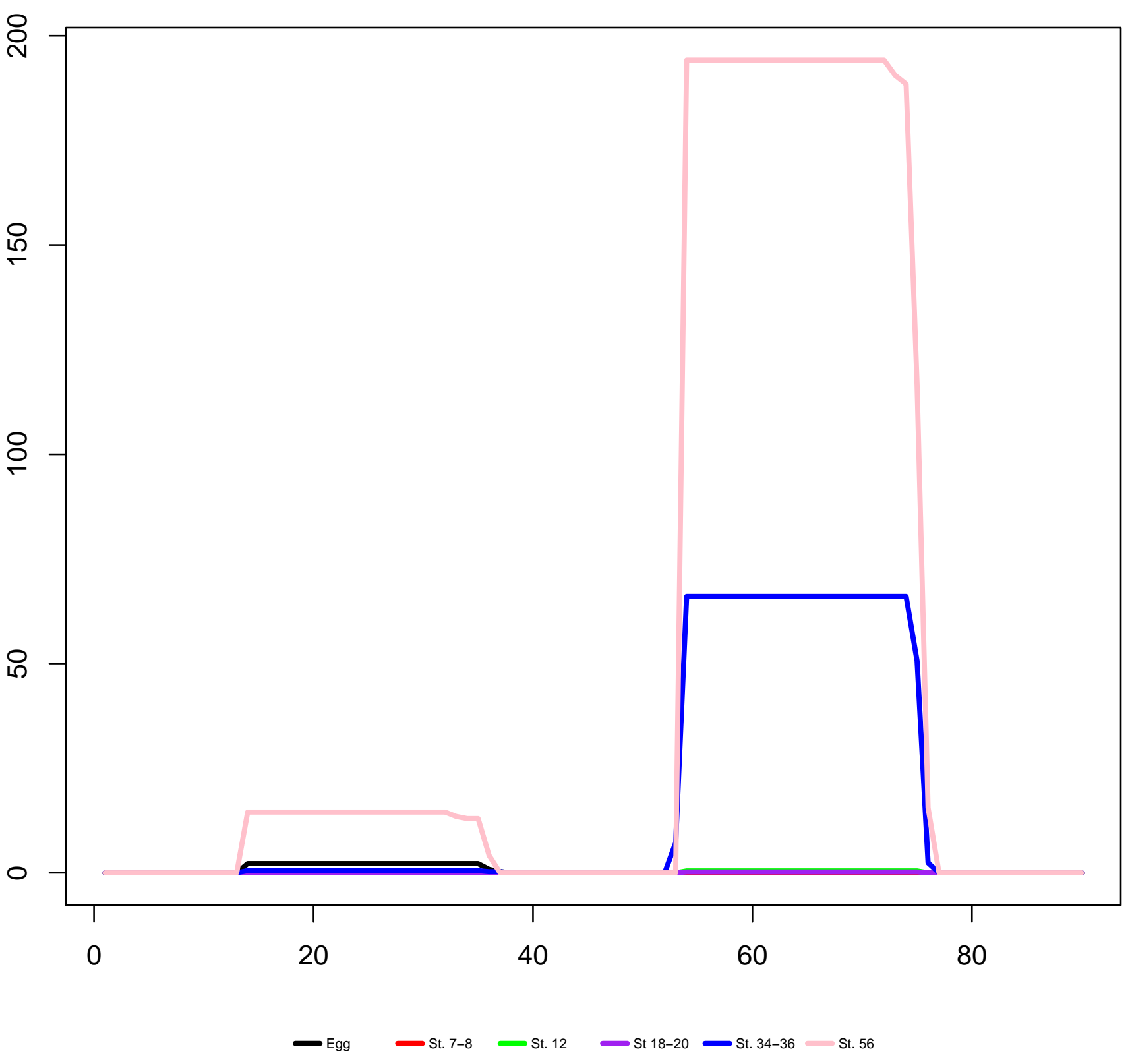


Scaffold34765_99056-99138(-) mir-124a-1

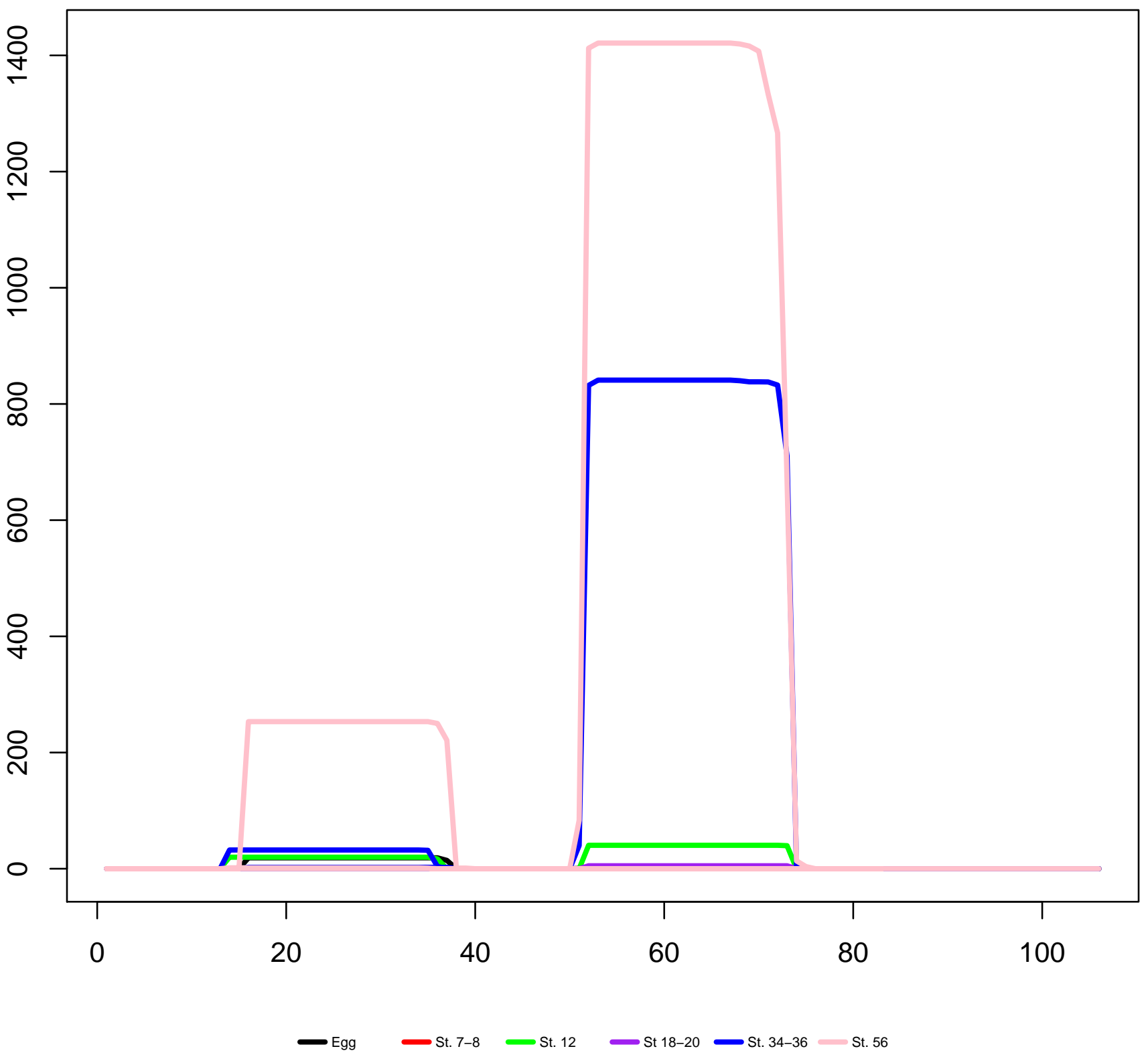


Scaffold35504_17926-18023(-) mir-140

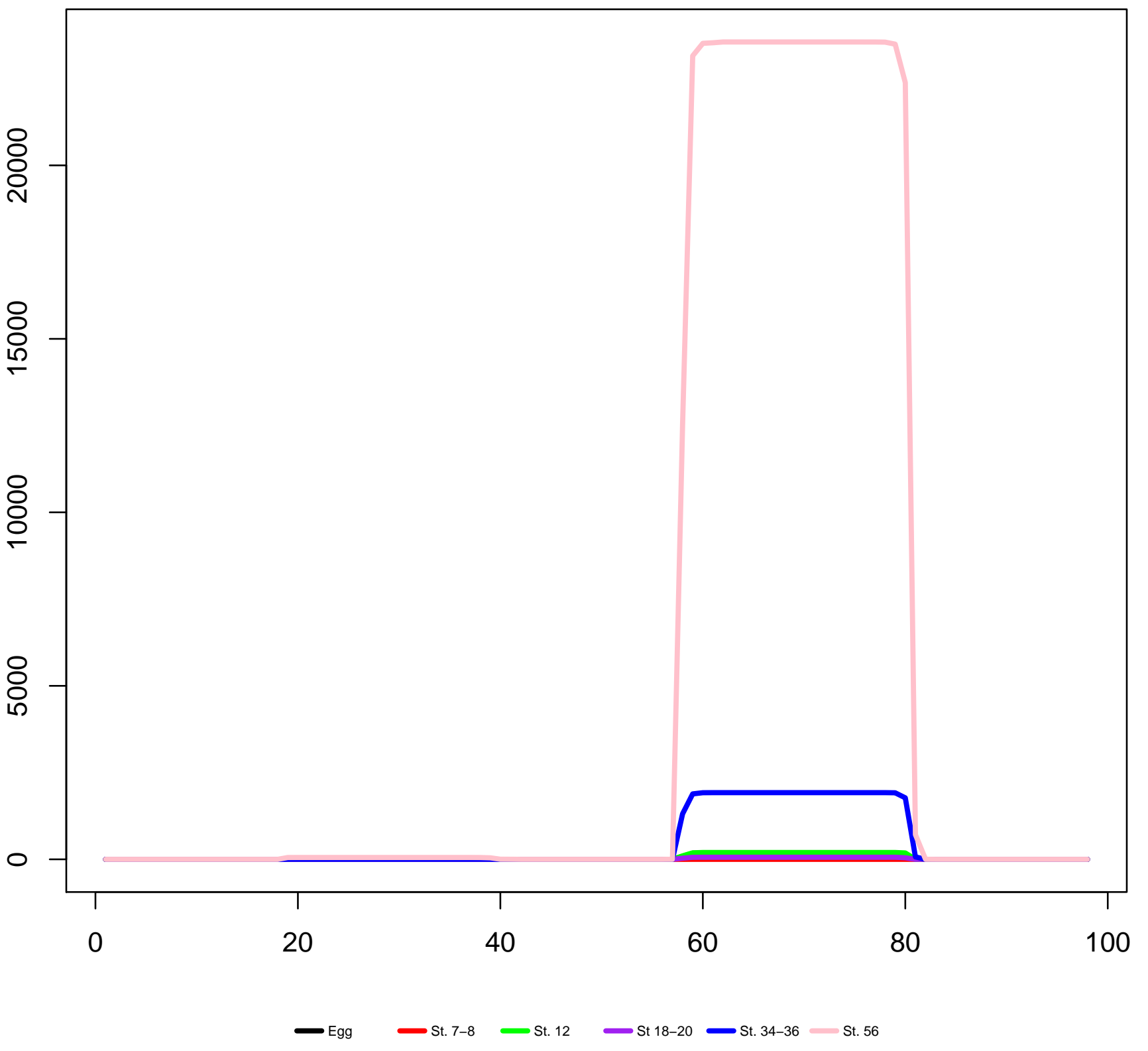


Scaffold35676_1034447-1034530(+) mir-1a-1

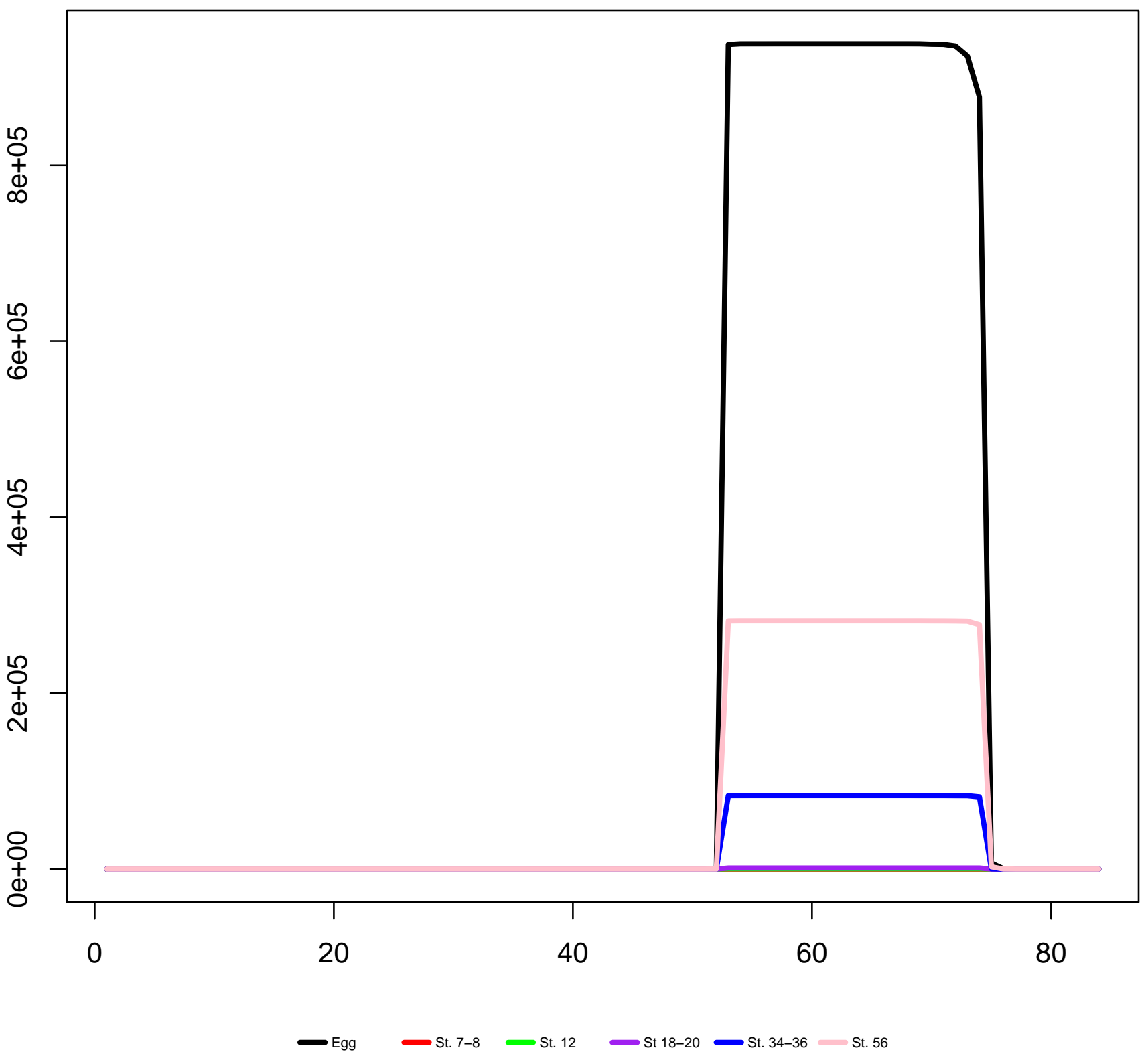


Scaffold35768_338807-338876(-) mir-375

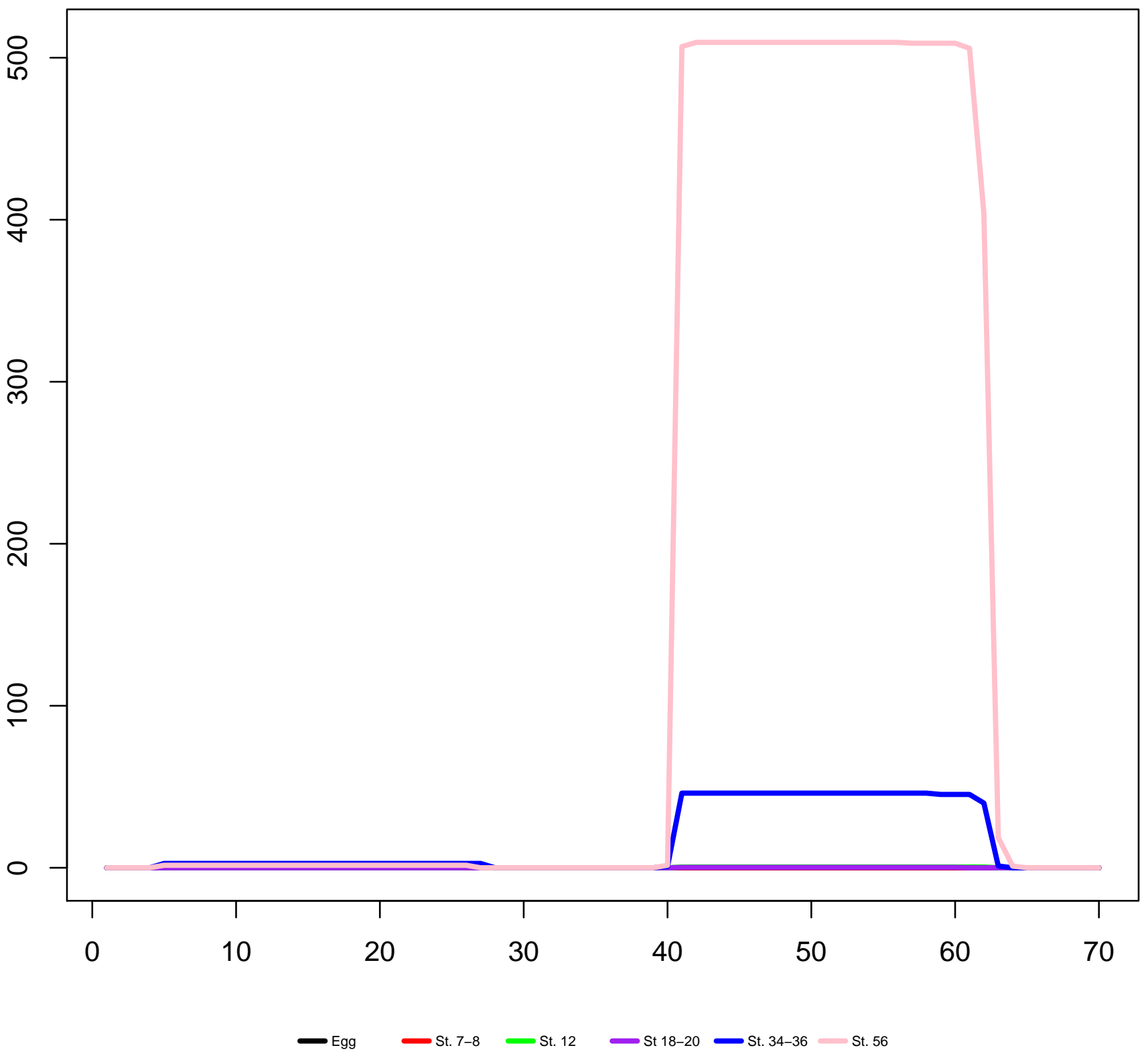




\section{Scaffold35768_1553077-1553158(-) mir-128-2}

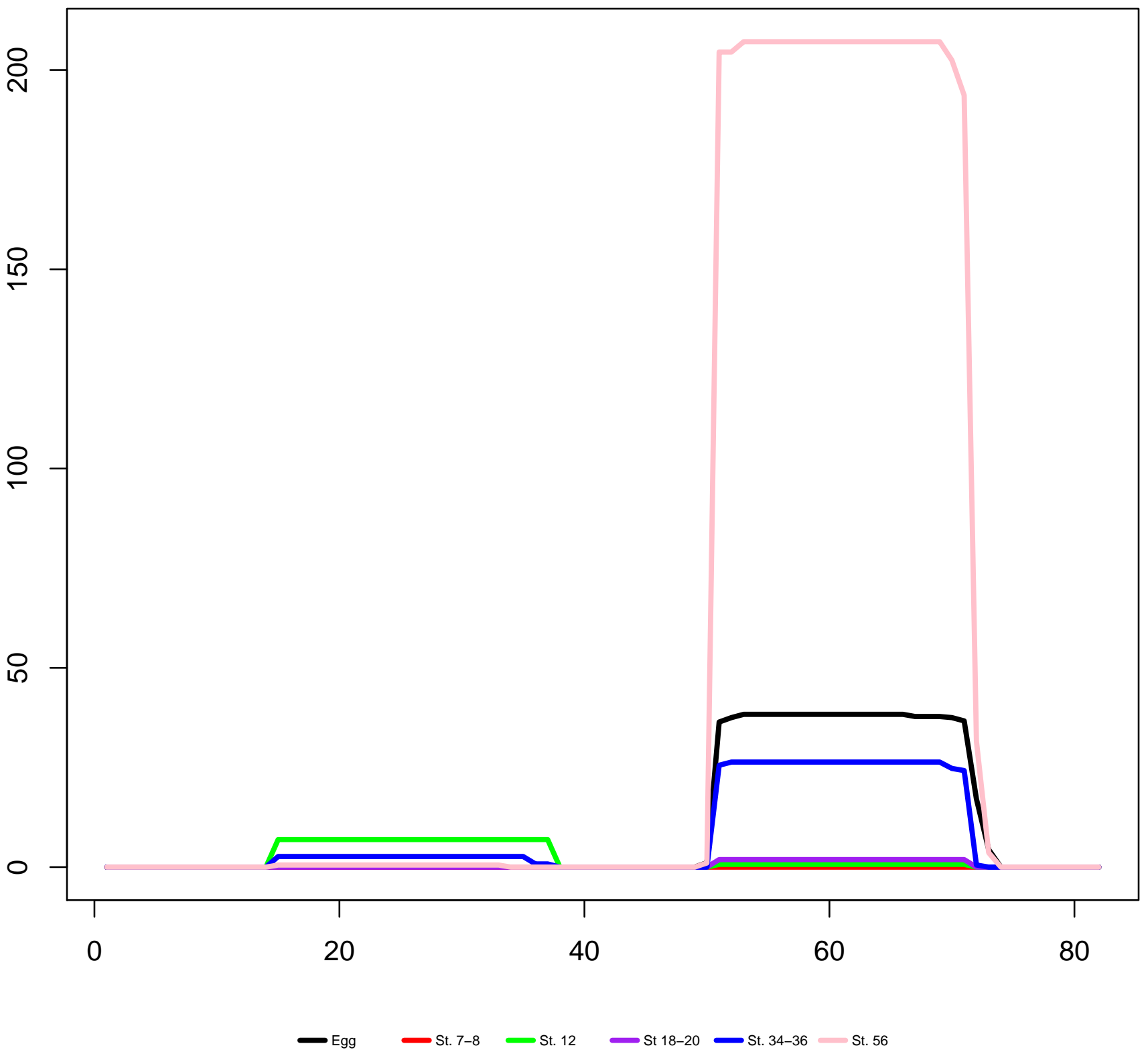


Scaffold35984_159128-159219(-) mir-129-2

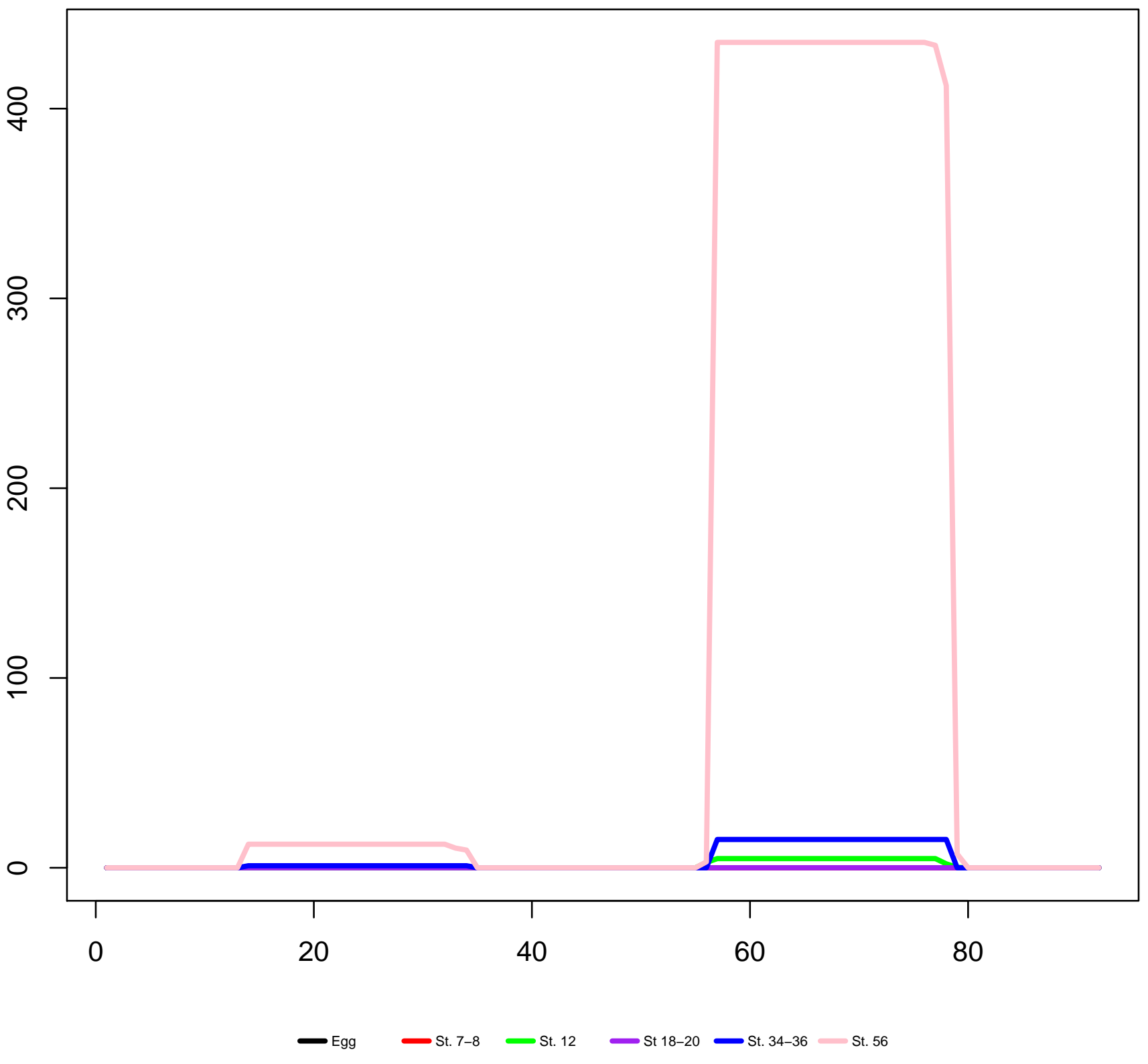


Scaffold36880_959439-959500(+) mir-1b

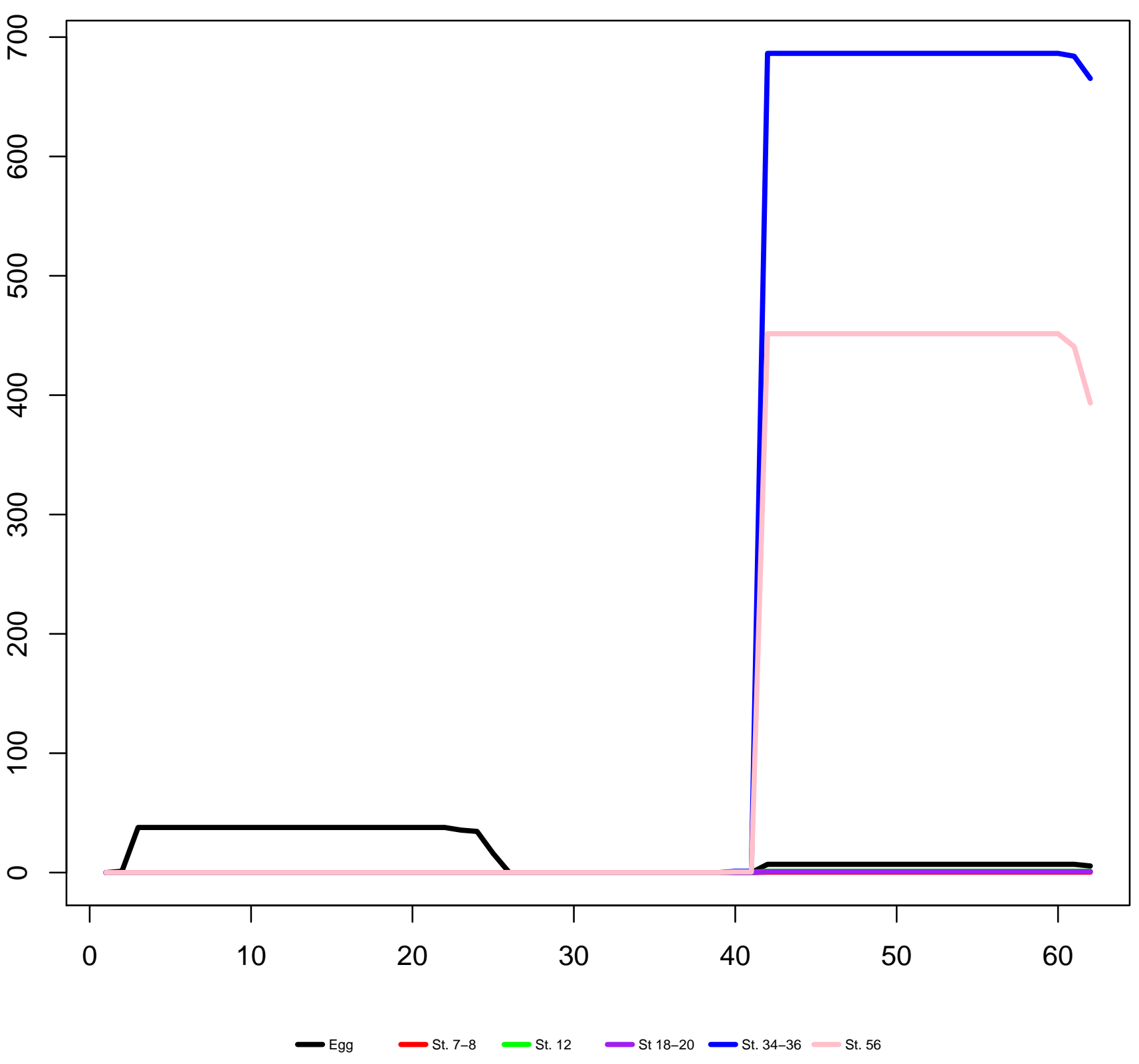


Scaffold36880_959852-959927(+) mir-133d

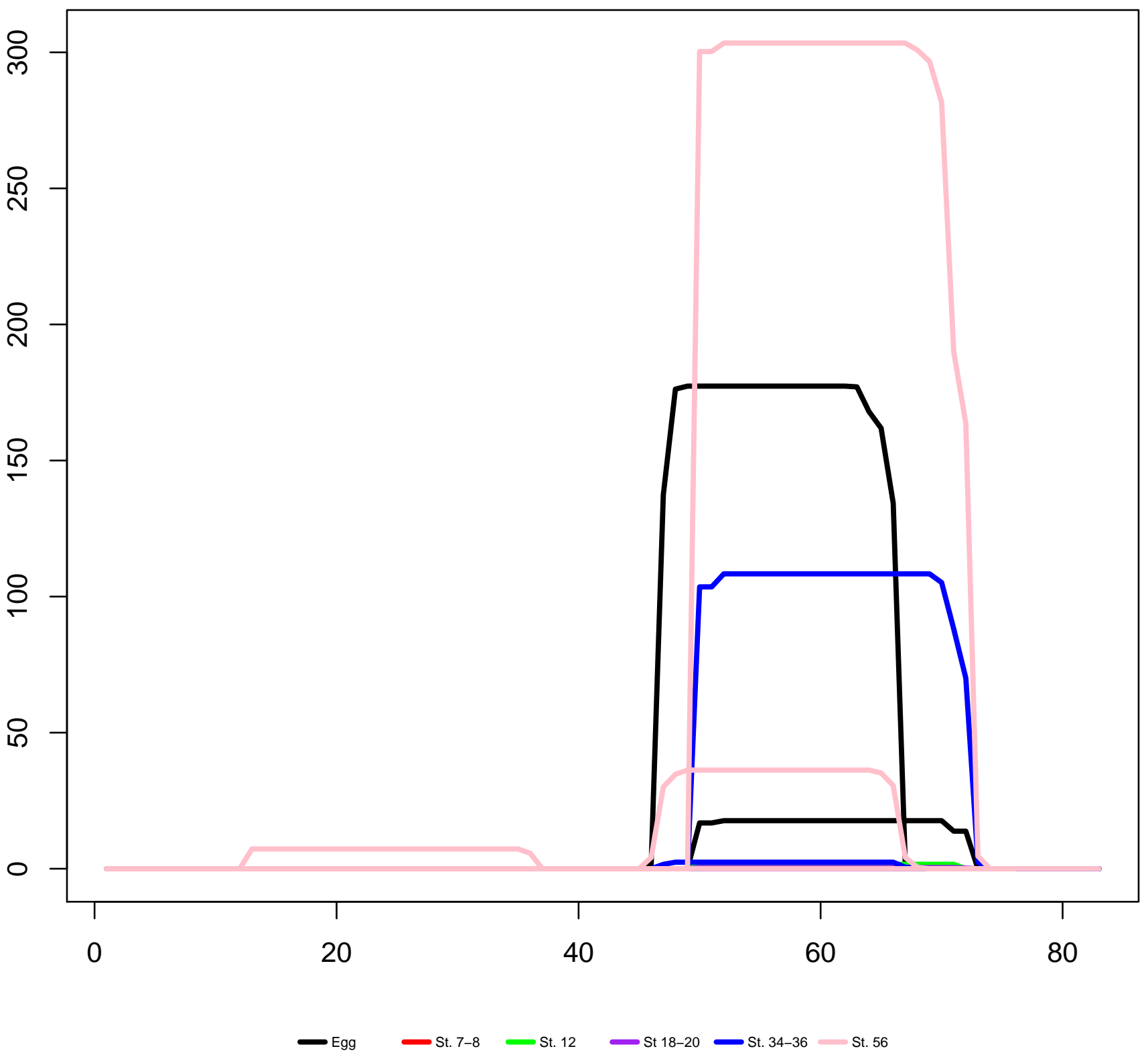


Scaffold37142_1-98(+) mir-9-1

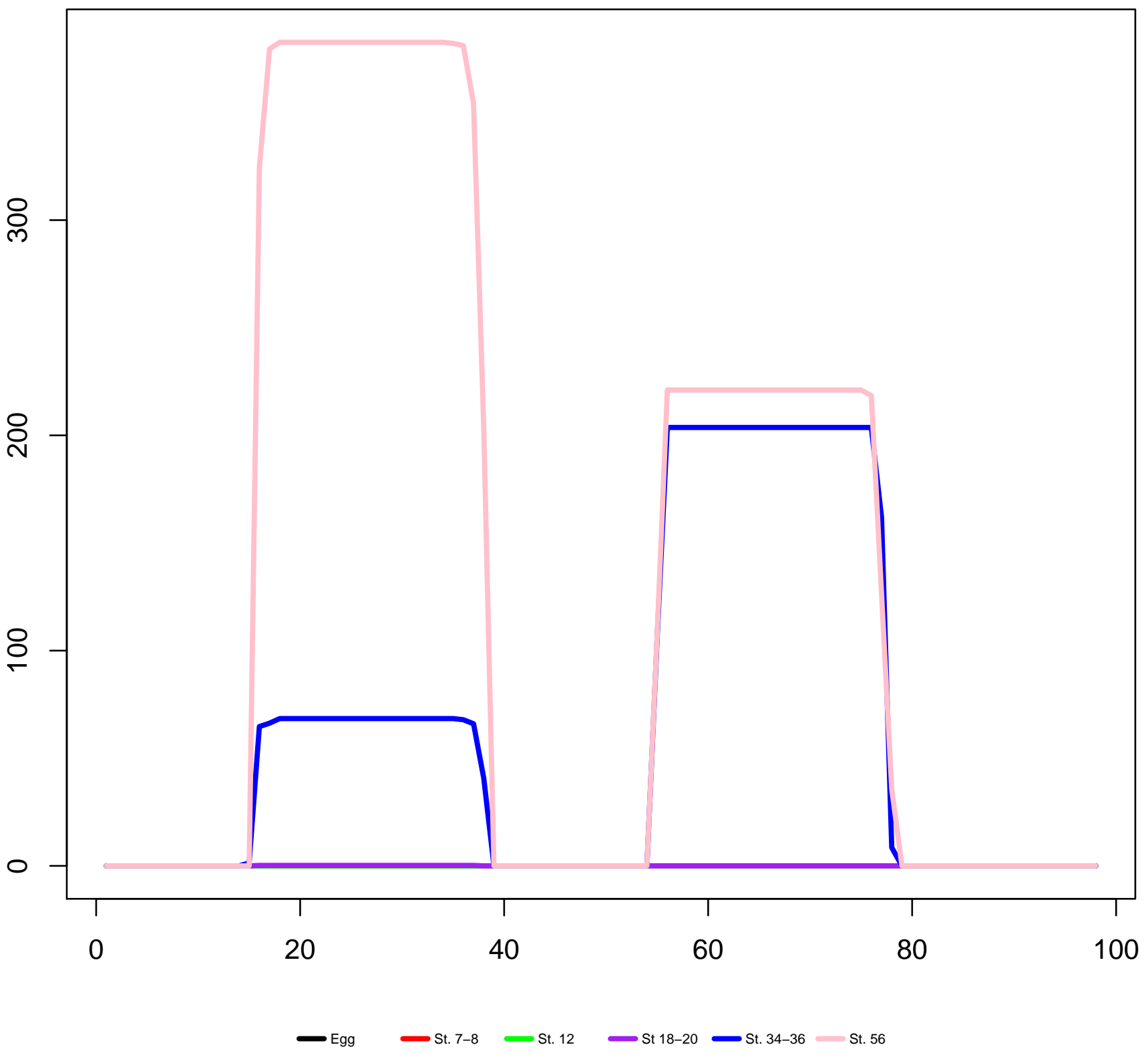


Scaffold37636_123495-123576(-) mir-460

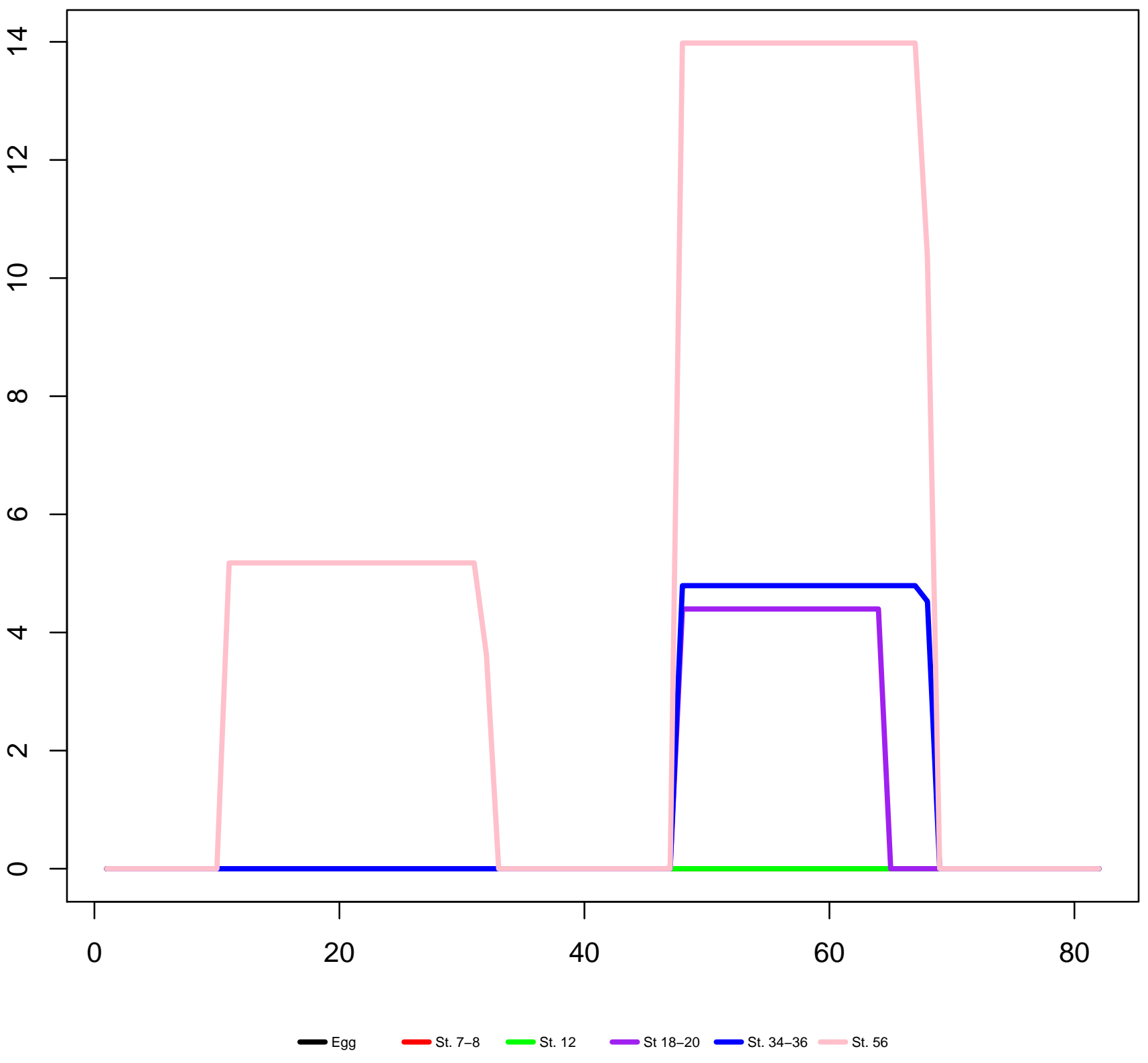


Scaffold38016_408721-408788(+) mir-302

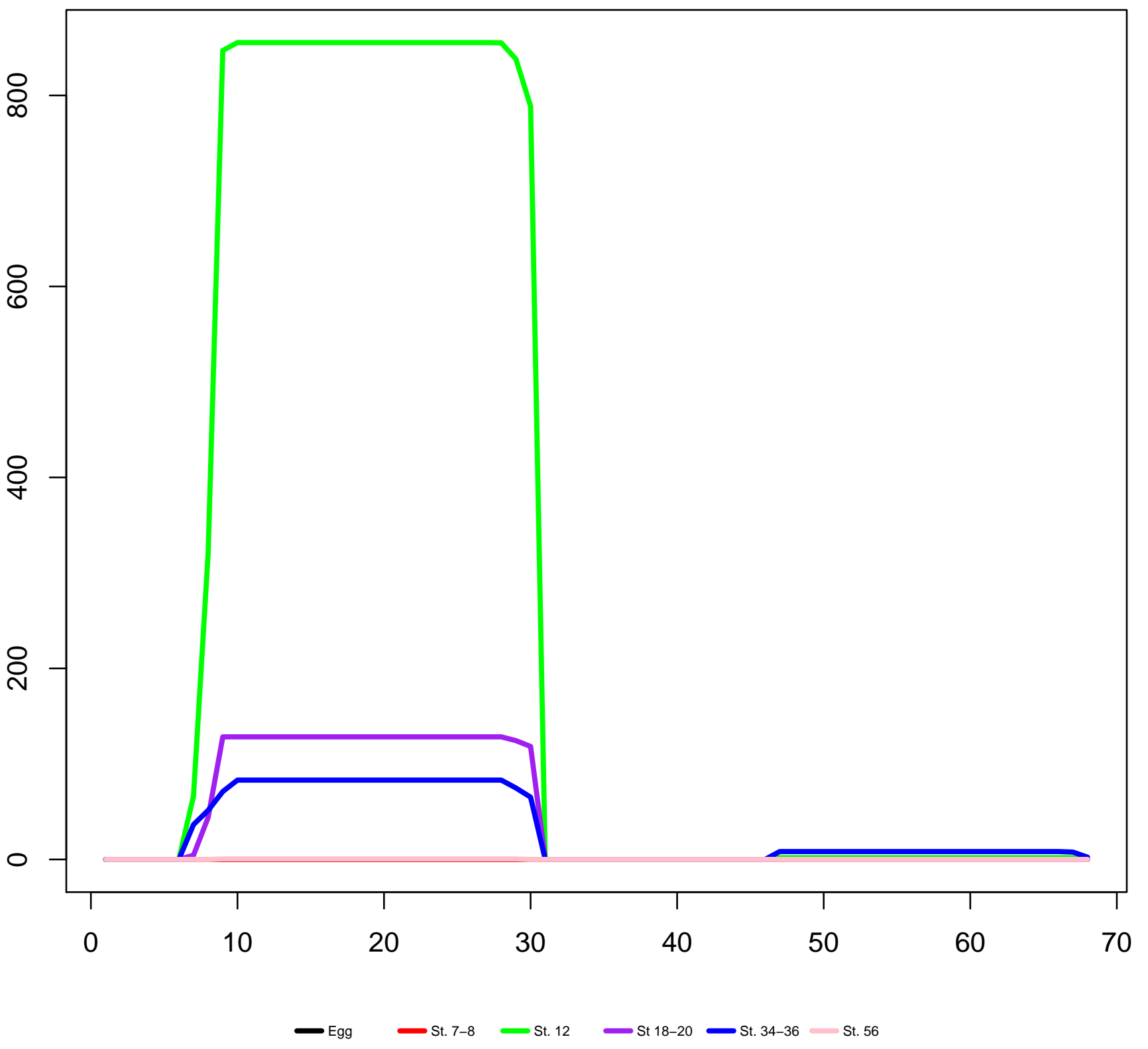


Scaffold38348_47927-48009(+) mir-103

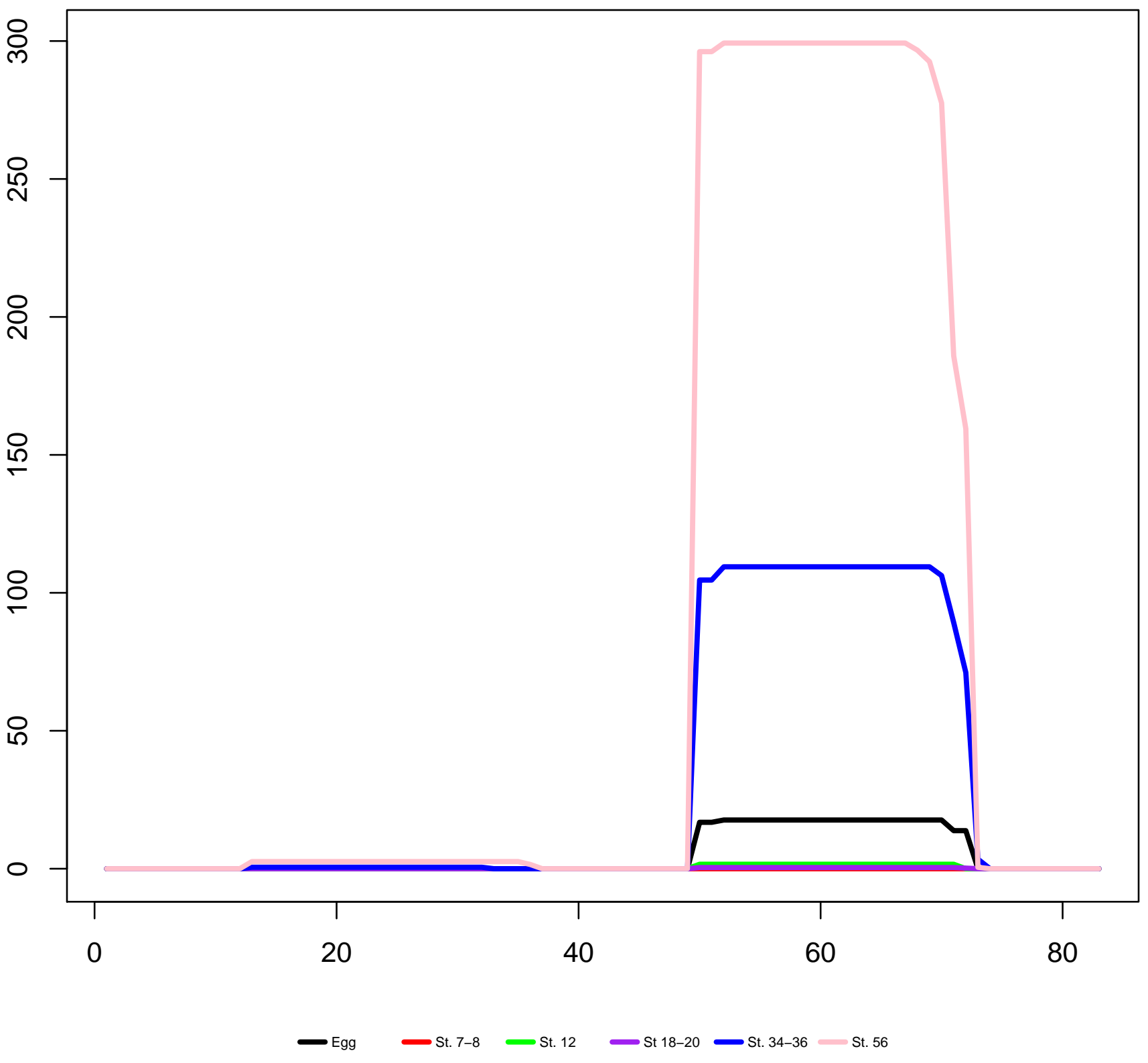


Scaffold38718_607987-608073(-) mir-202

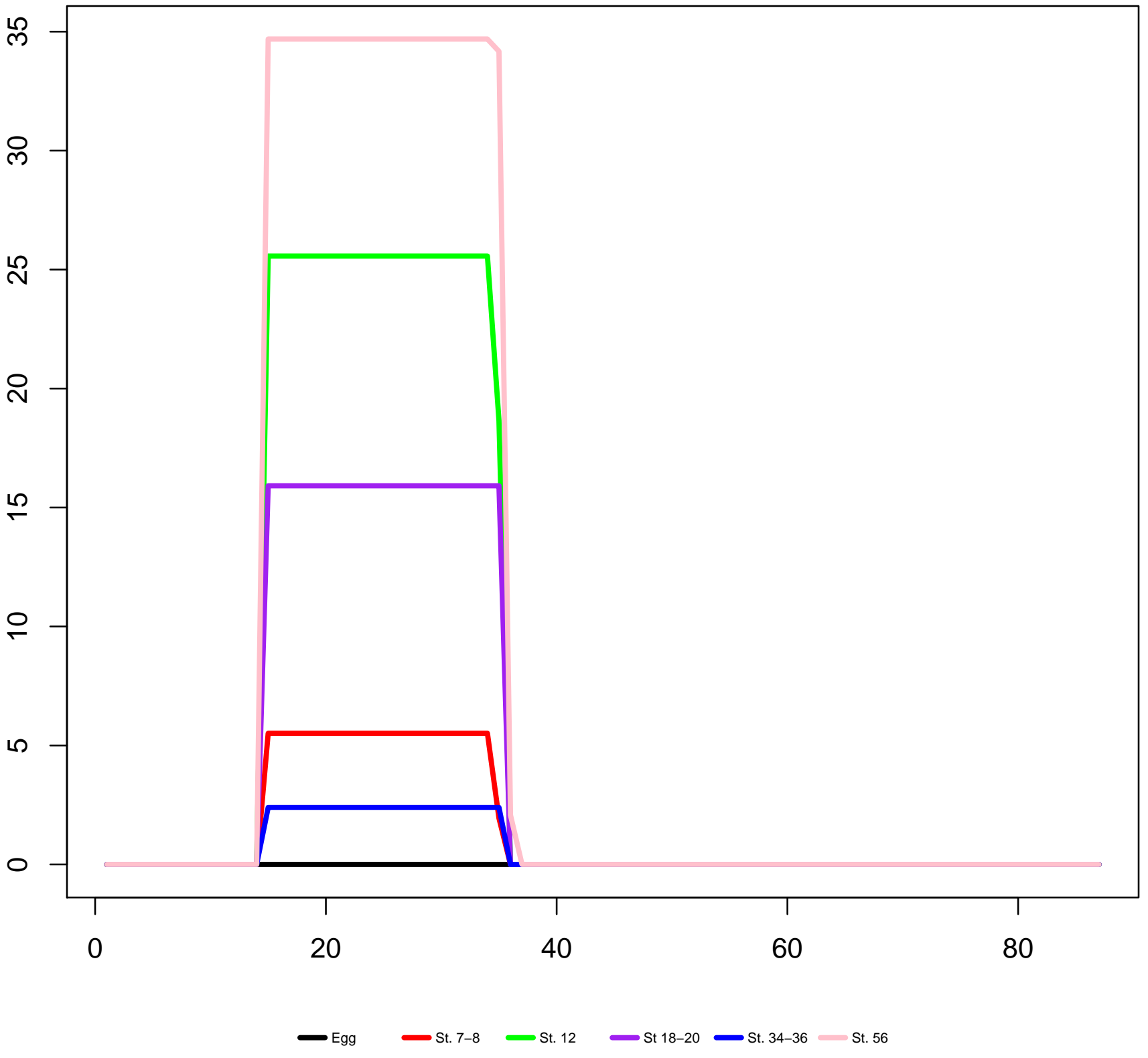


Scaffold38835_309080-309150(+) mir-338

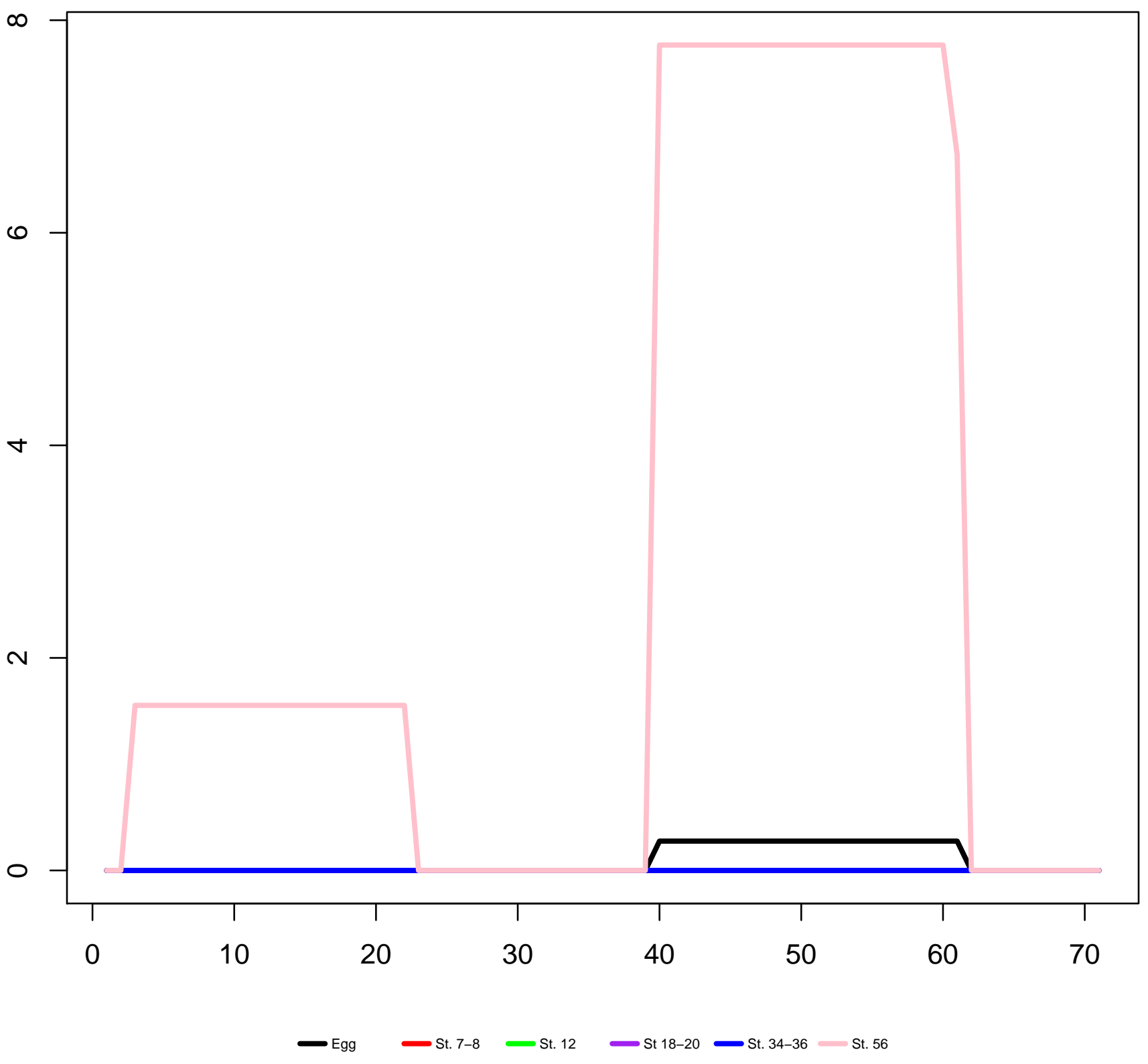


Scaffold38890_44381-44466(+) mir-142

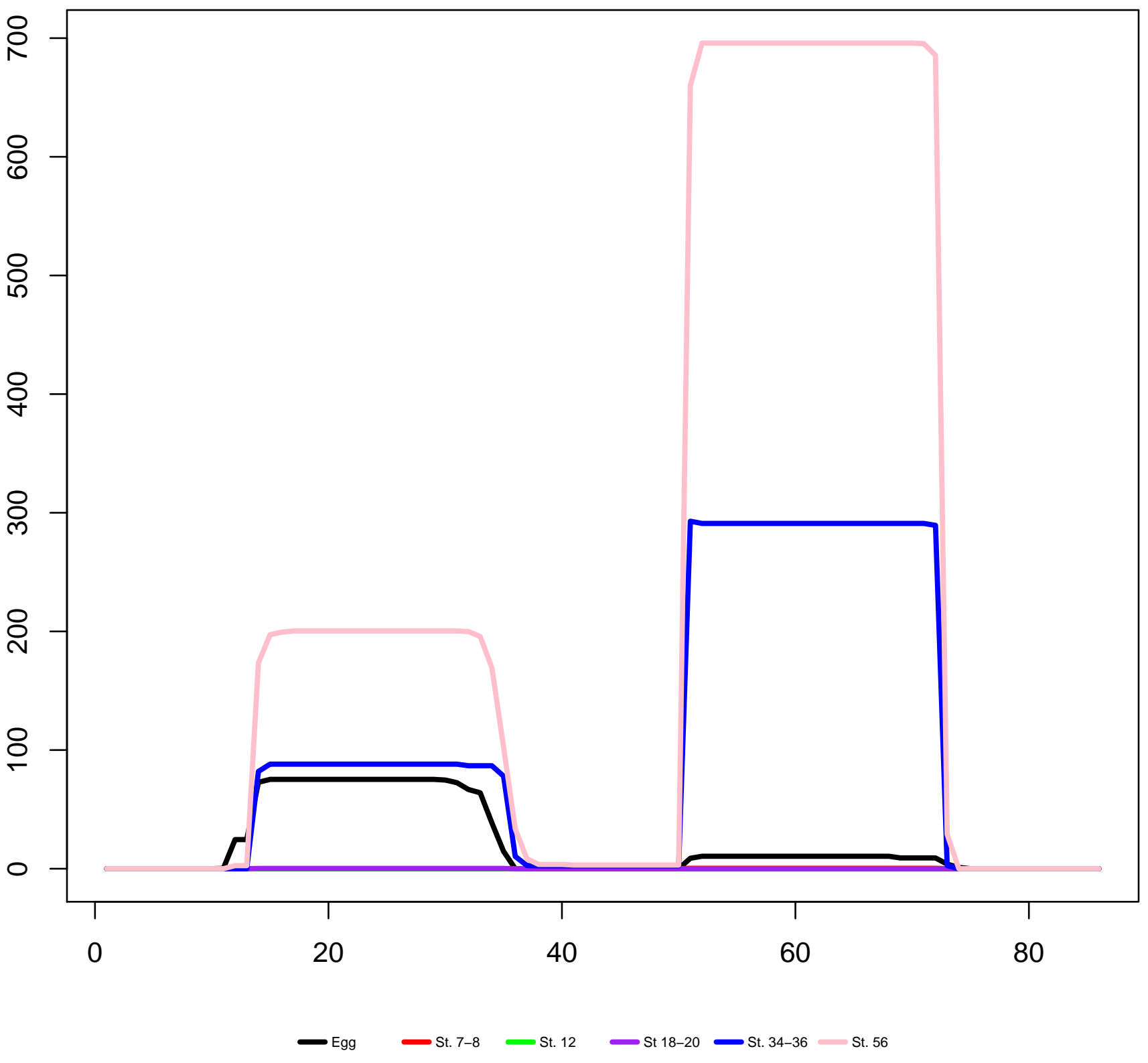


Scaffold39146_153469-153536(+) mir-24b

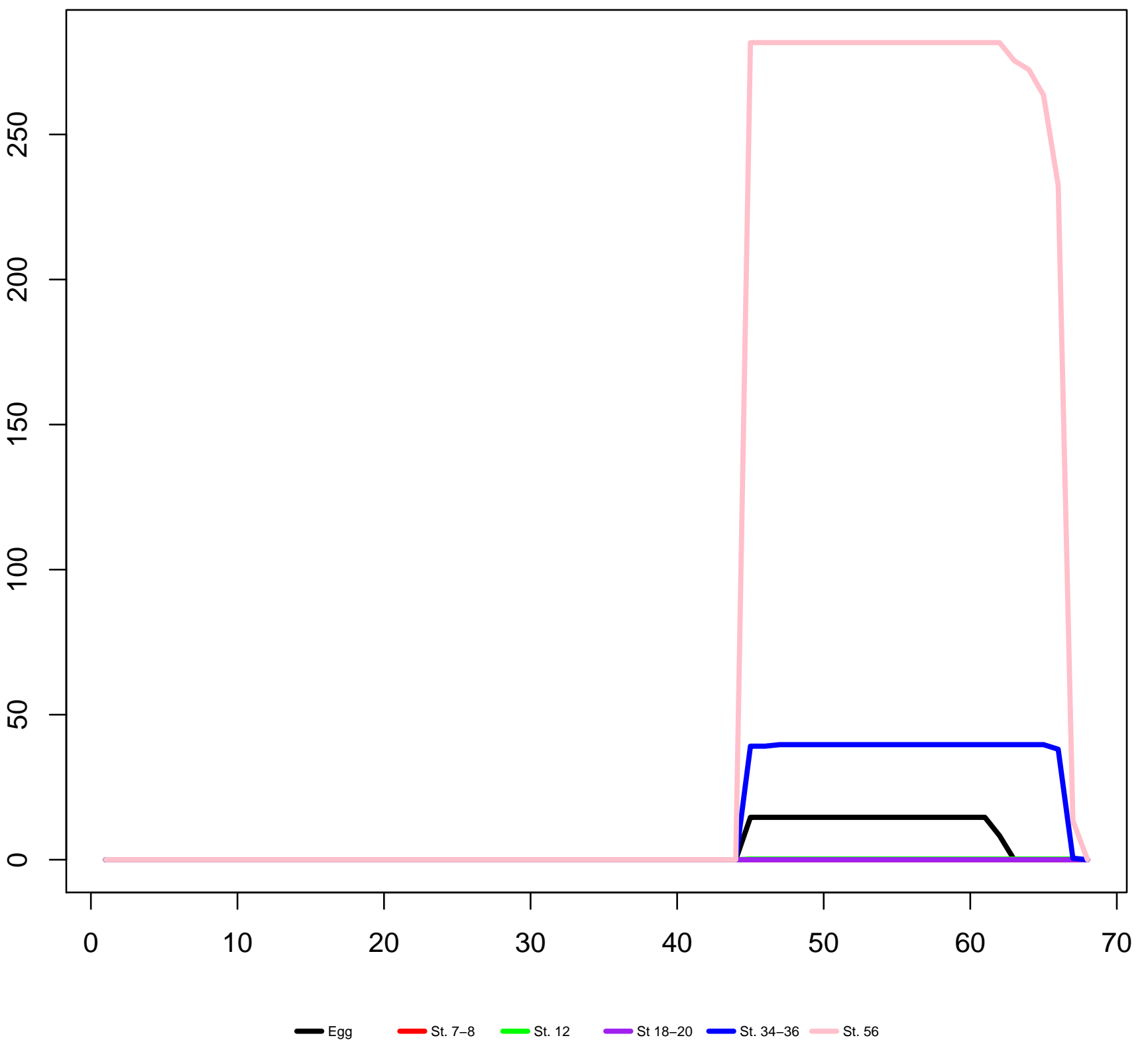


Scaffold393411_1-101(-) mir-137a

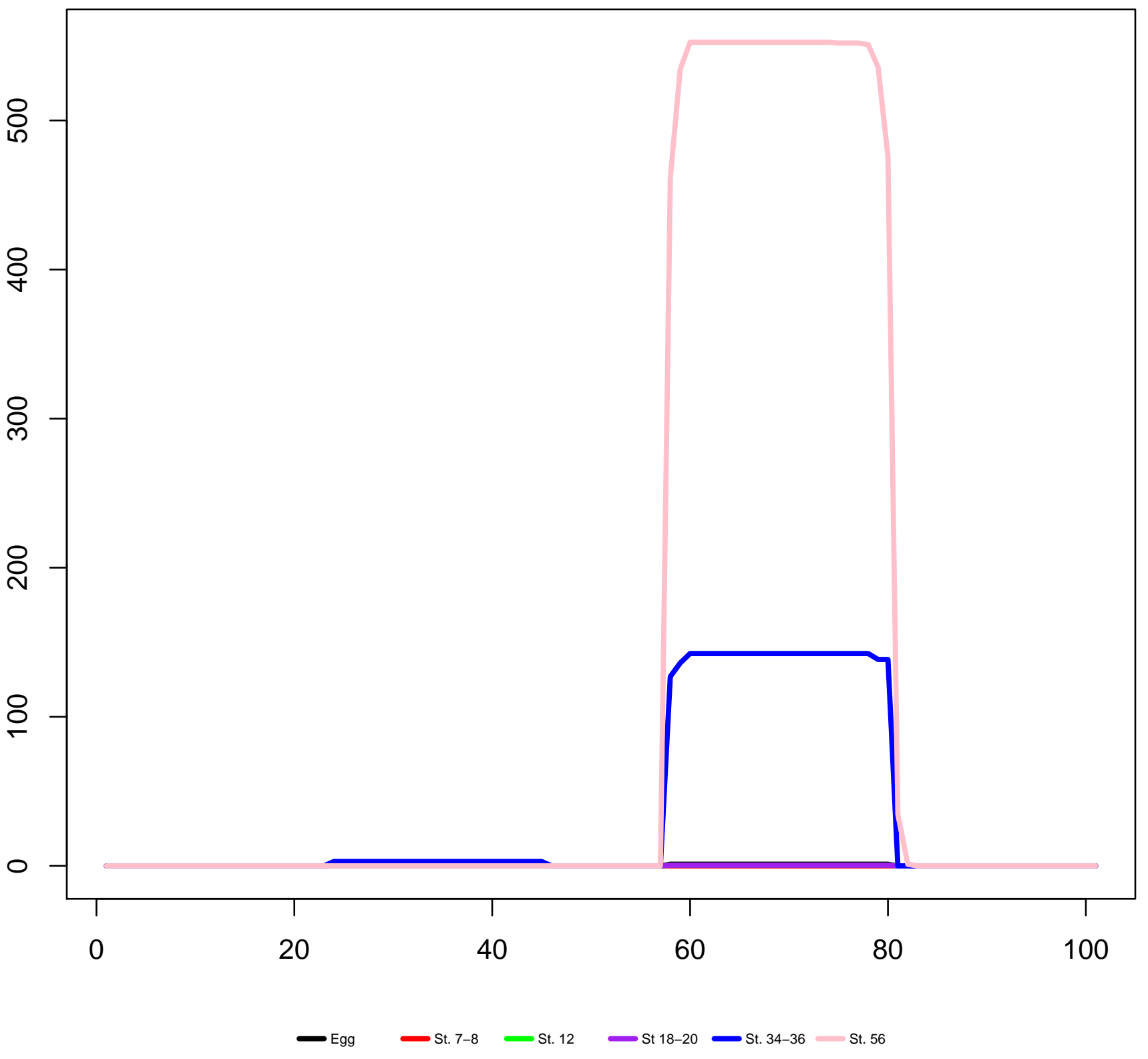


Scaffold39681_82041-82115(+) mir-23a-2

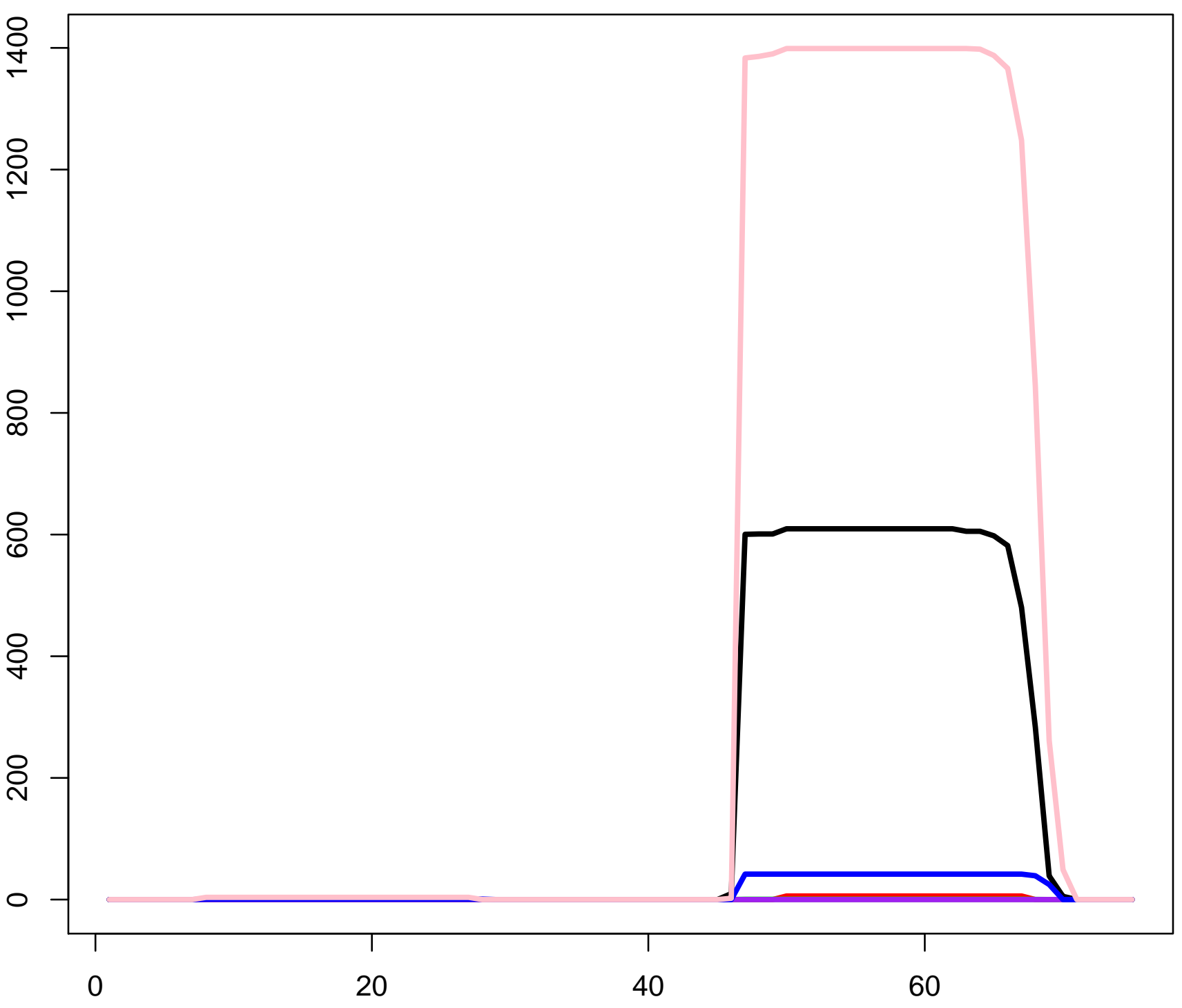


Scaffold39681_82330-82412(+) mir-27c-1

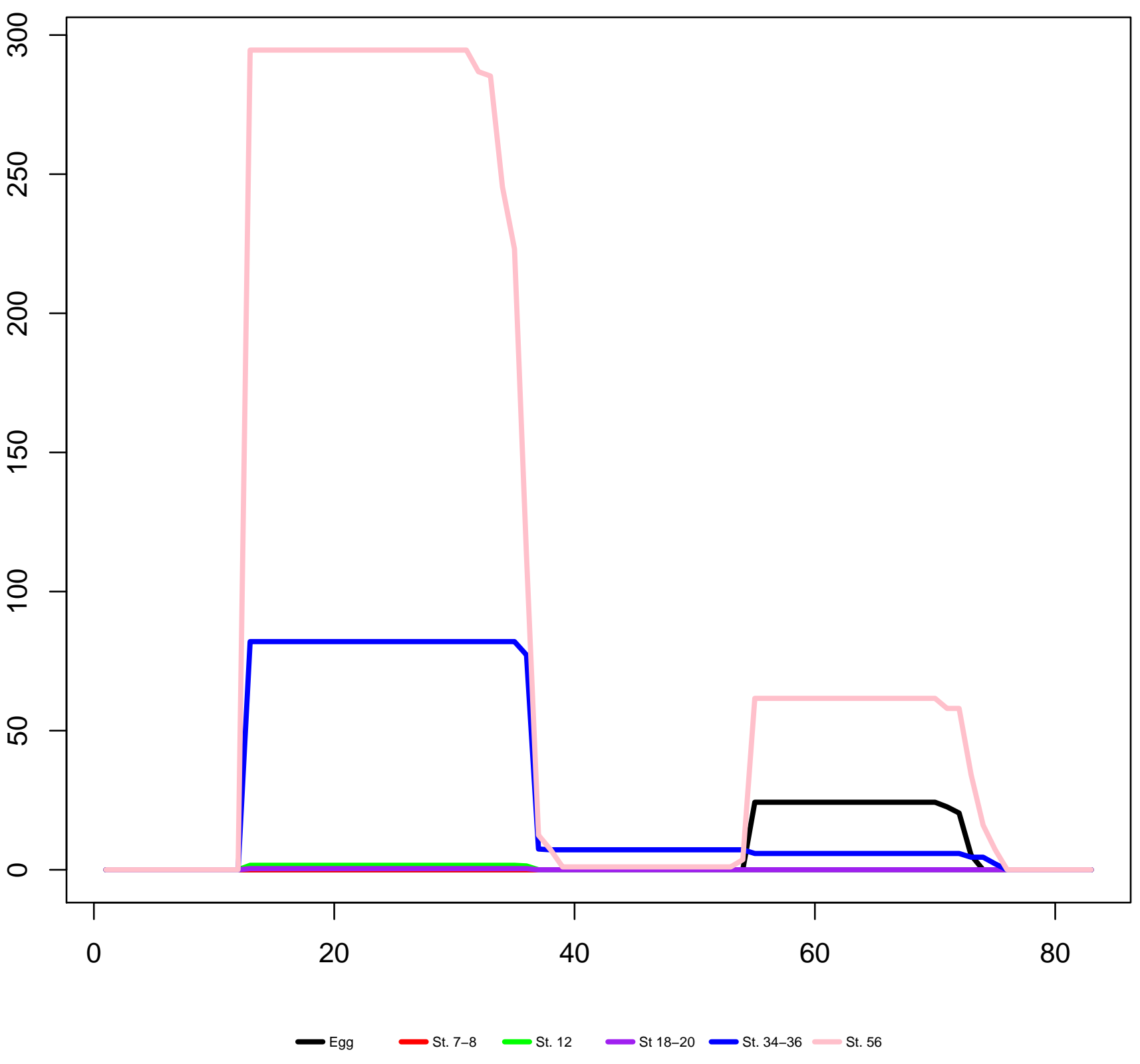


Scaffold402437_7-88(-) mir-124a-1

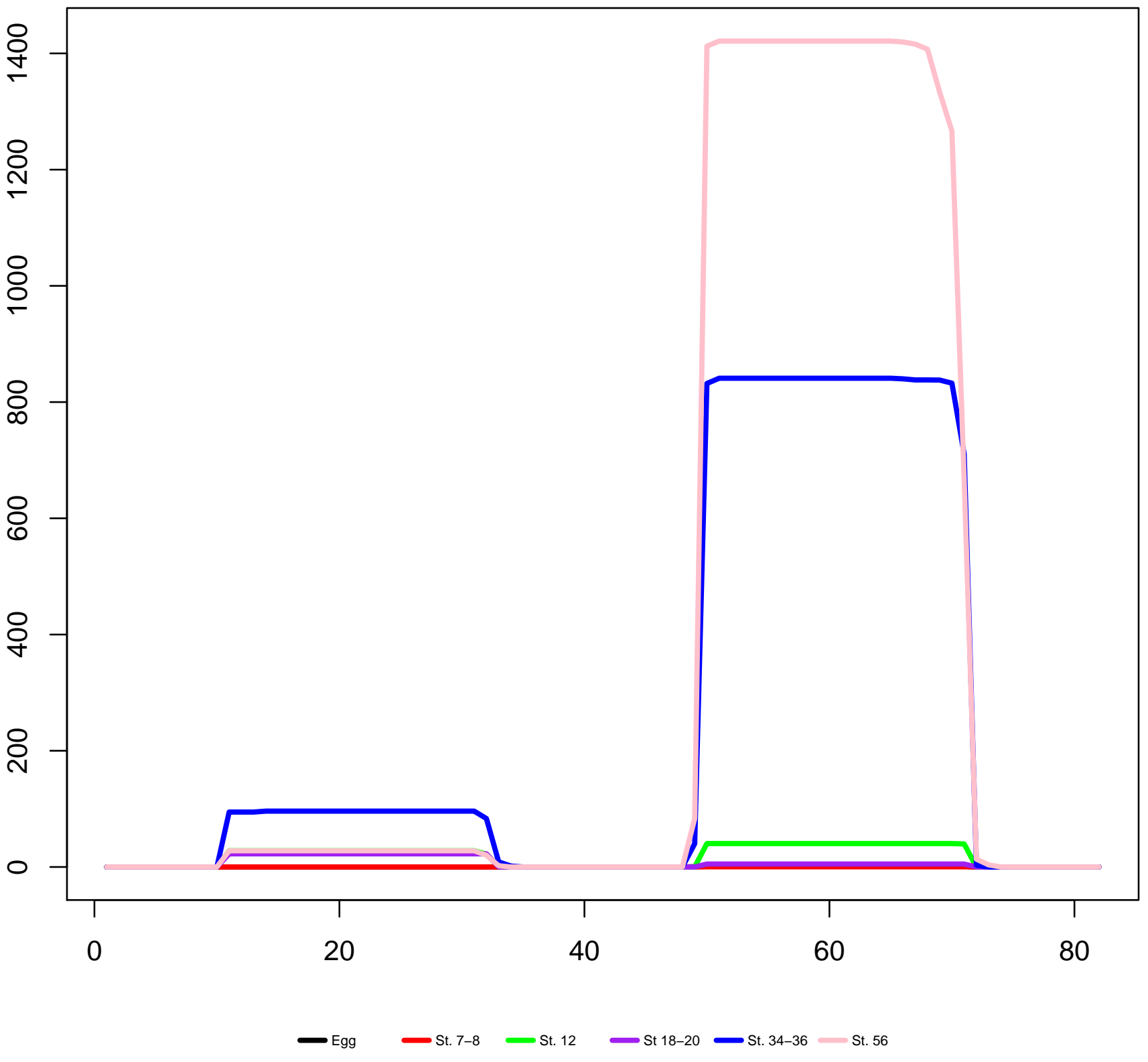


Scaffold40968_45511-45584(-) mir-1a-1

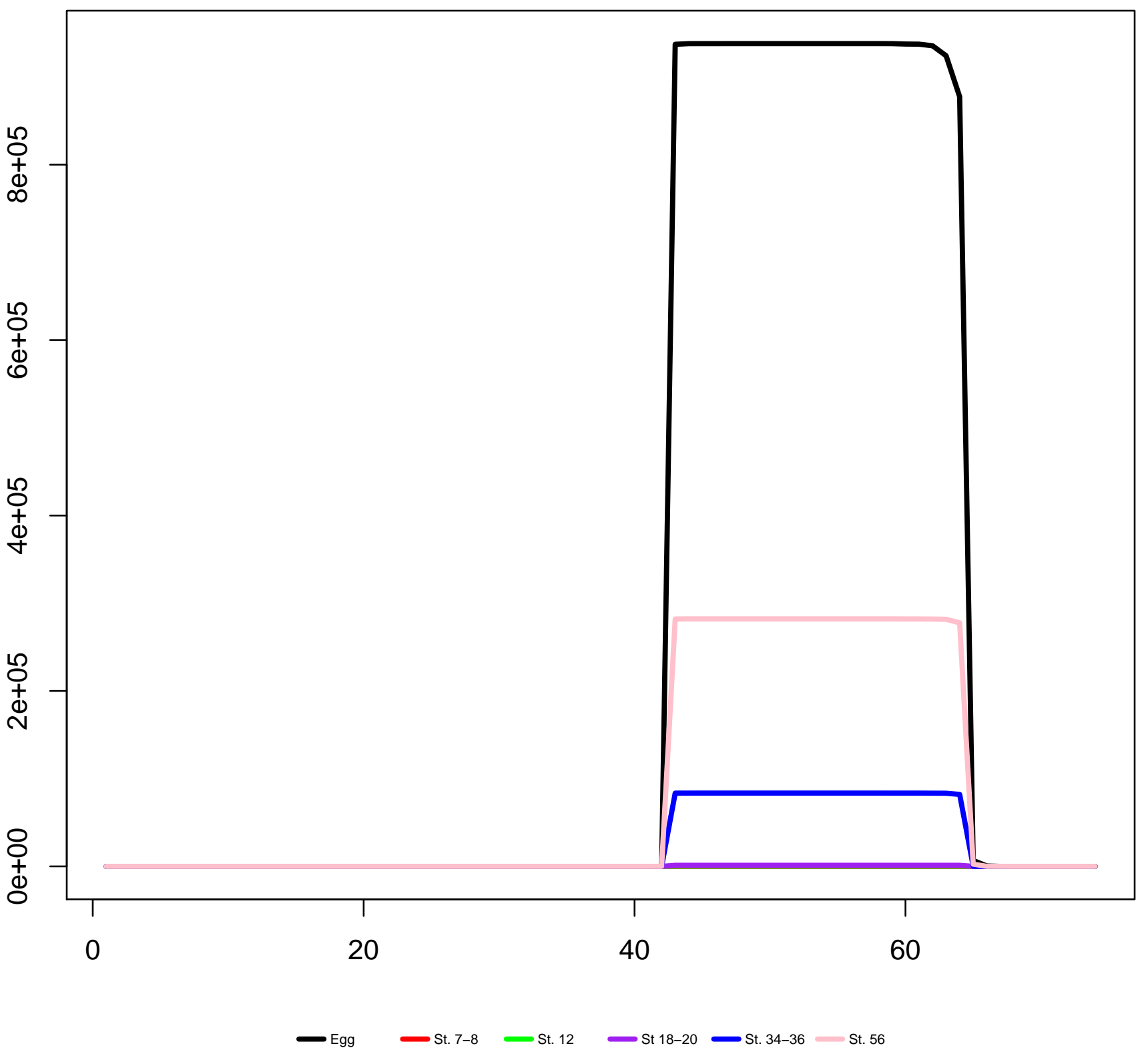


Scaffold41057_37-121(+) mir-1306

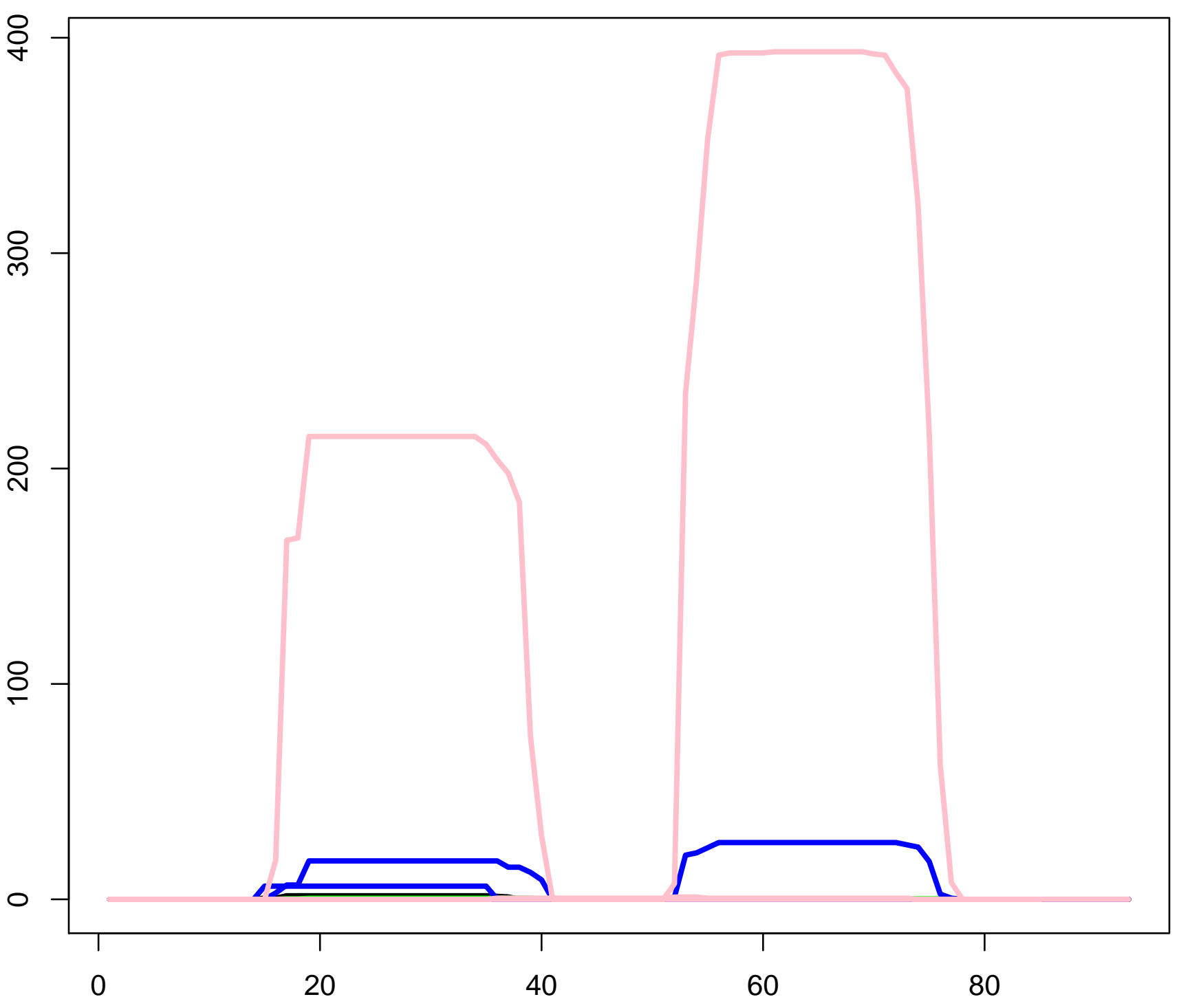


Scaffold4182_32726-32807(-) mir-124a-1

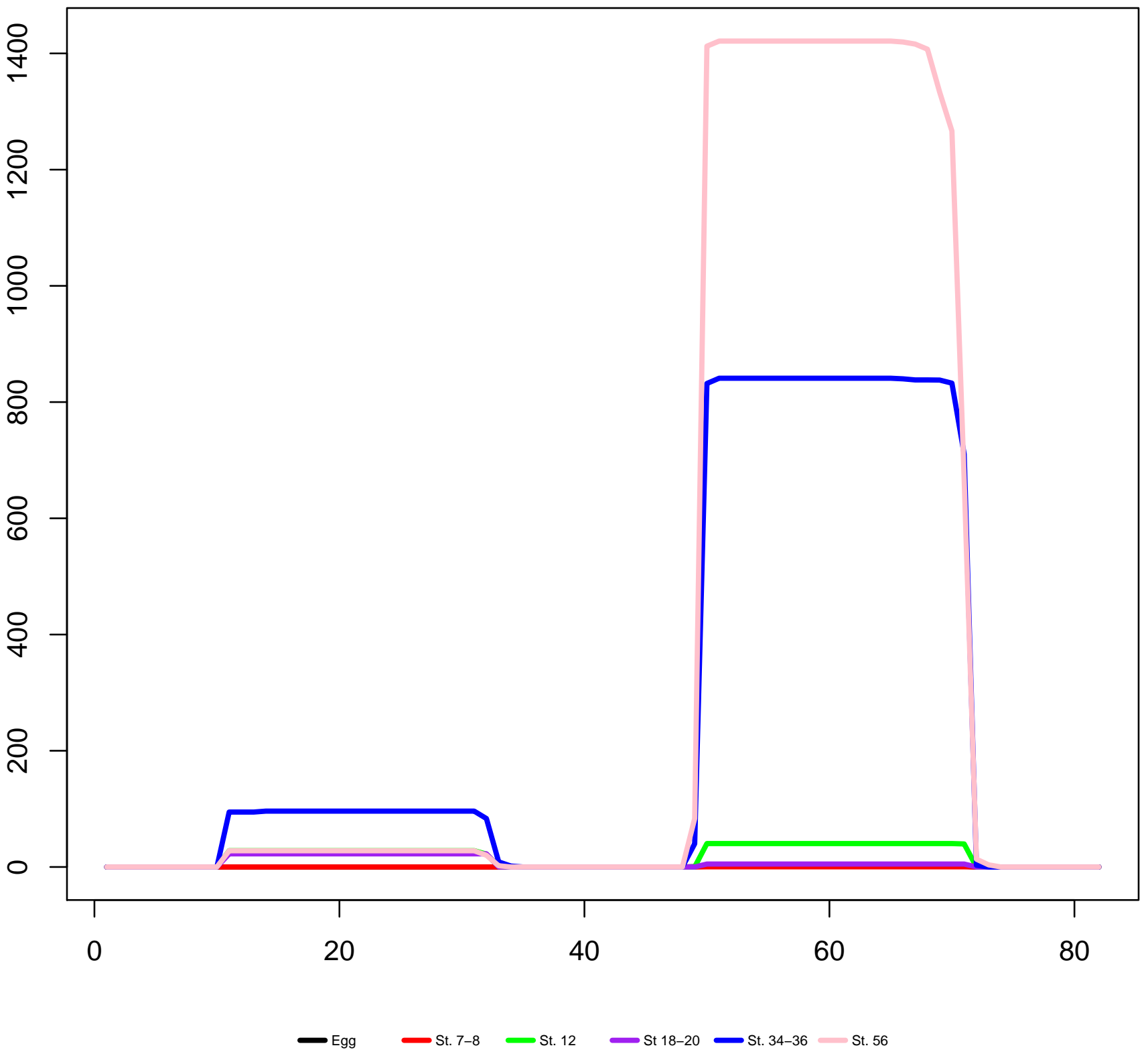


Scaffold42378_159328-159418(+) mir-200a

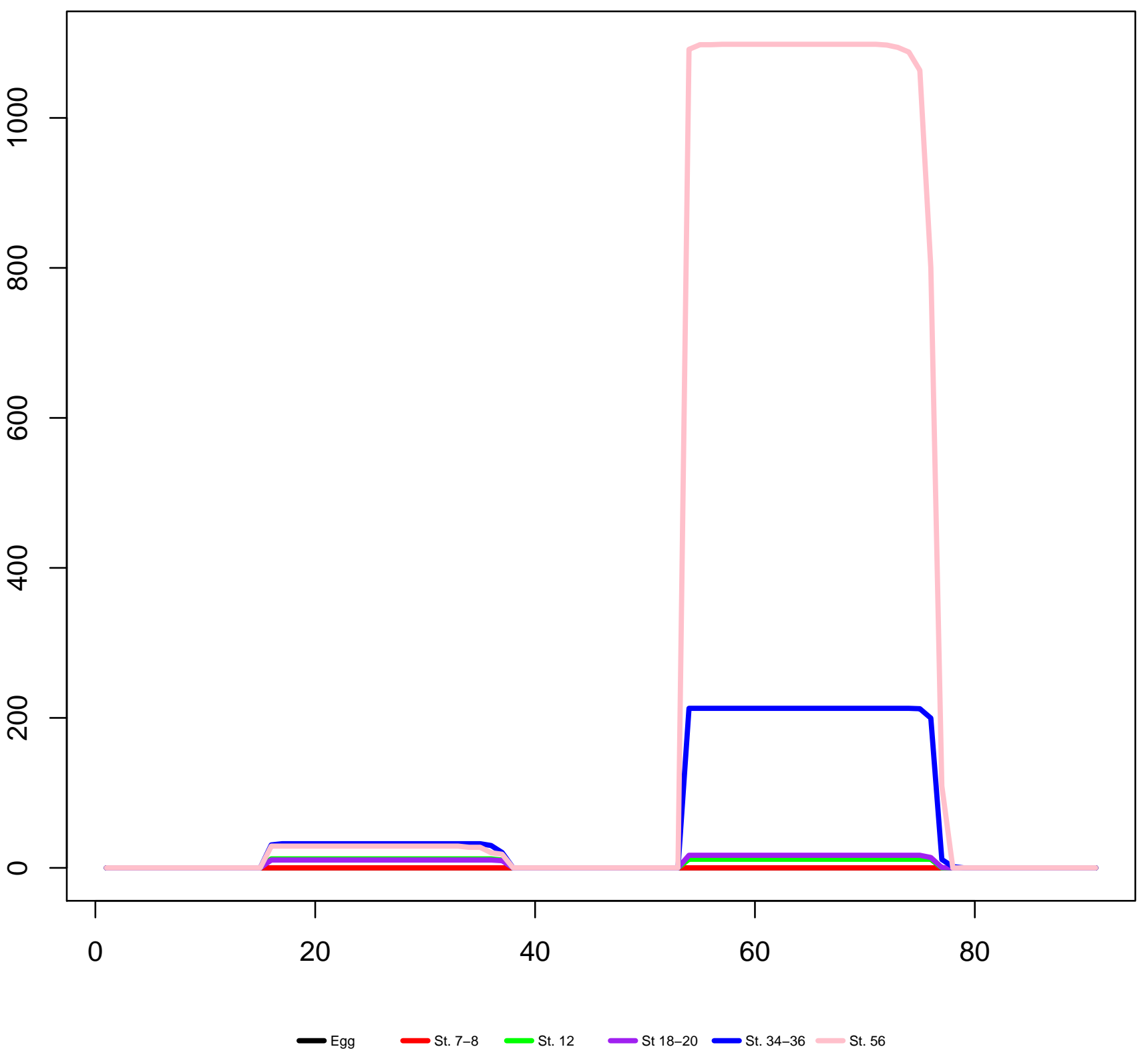


Scaffold42443_1411567-1411652(-) mir-29a-1

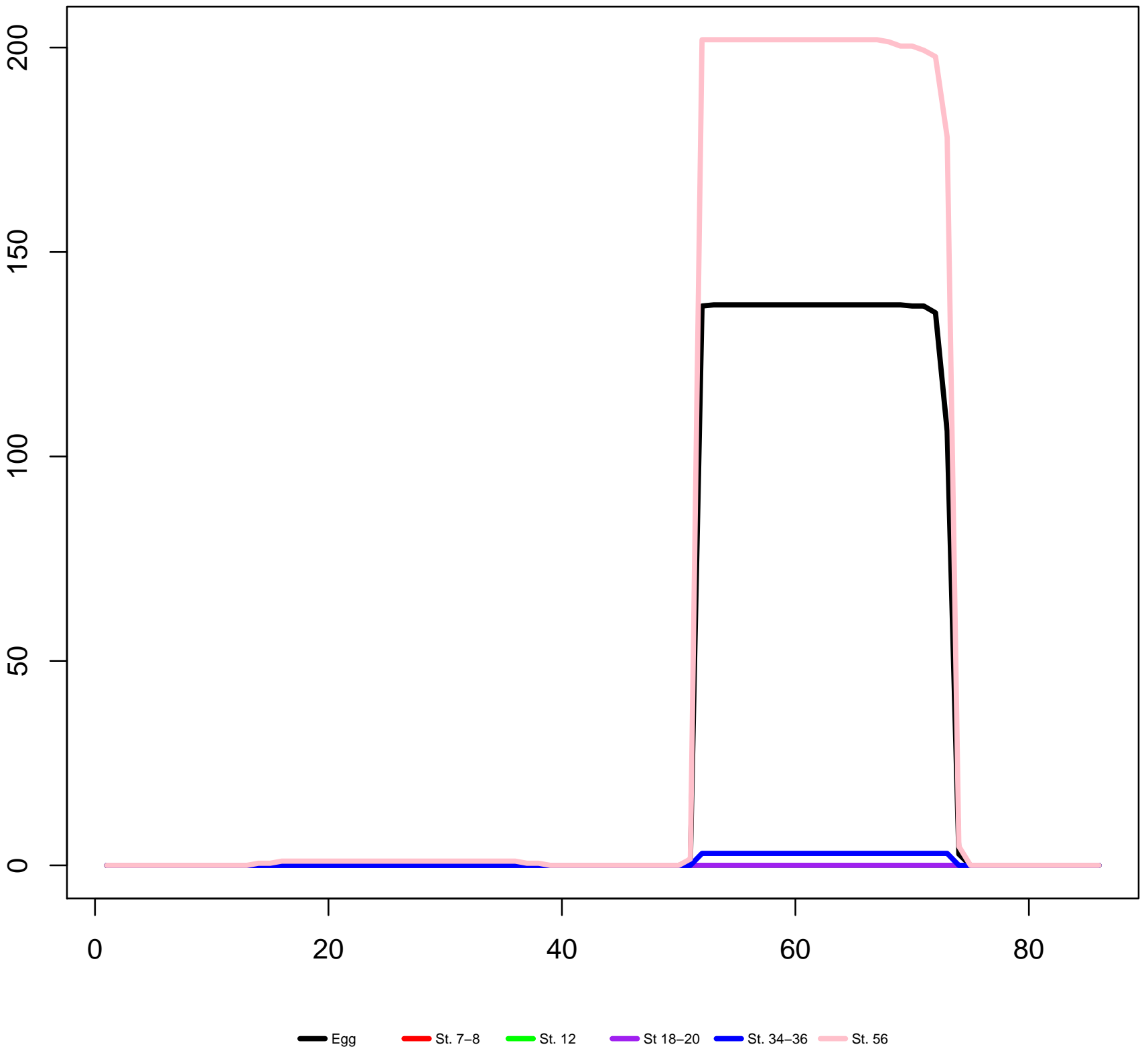


Scaffold43434_1066708-1066804(-) mir-10a

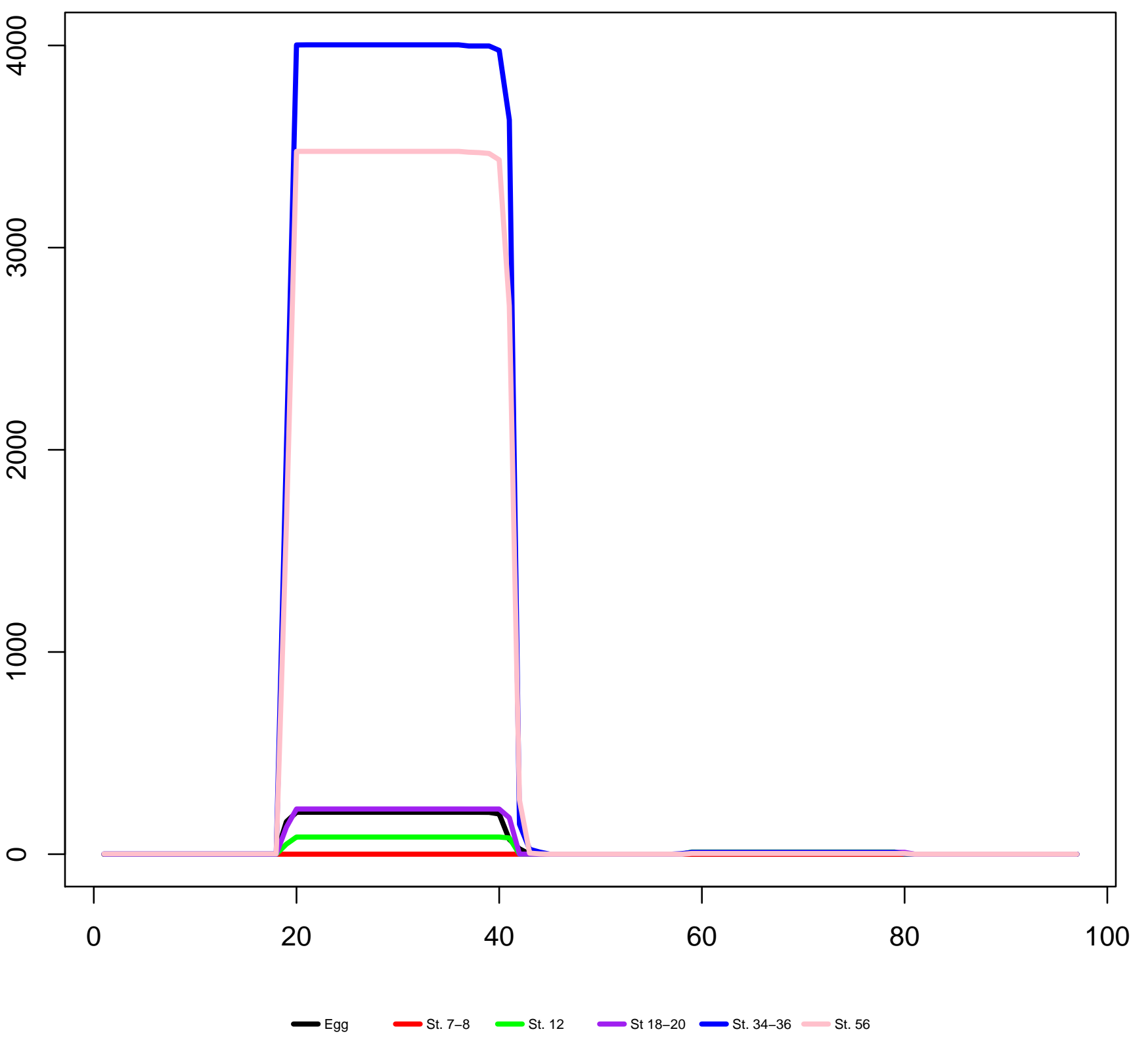


Scaffold436604_288799-288879(+) mir-208

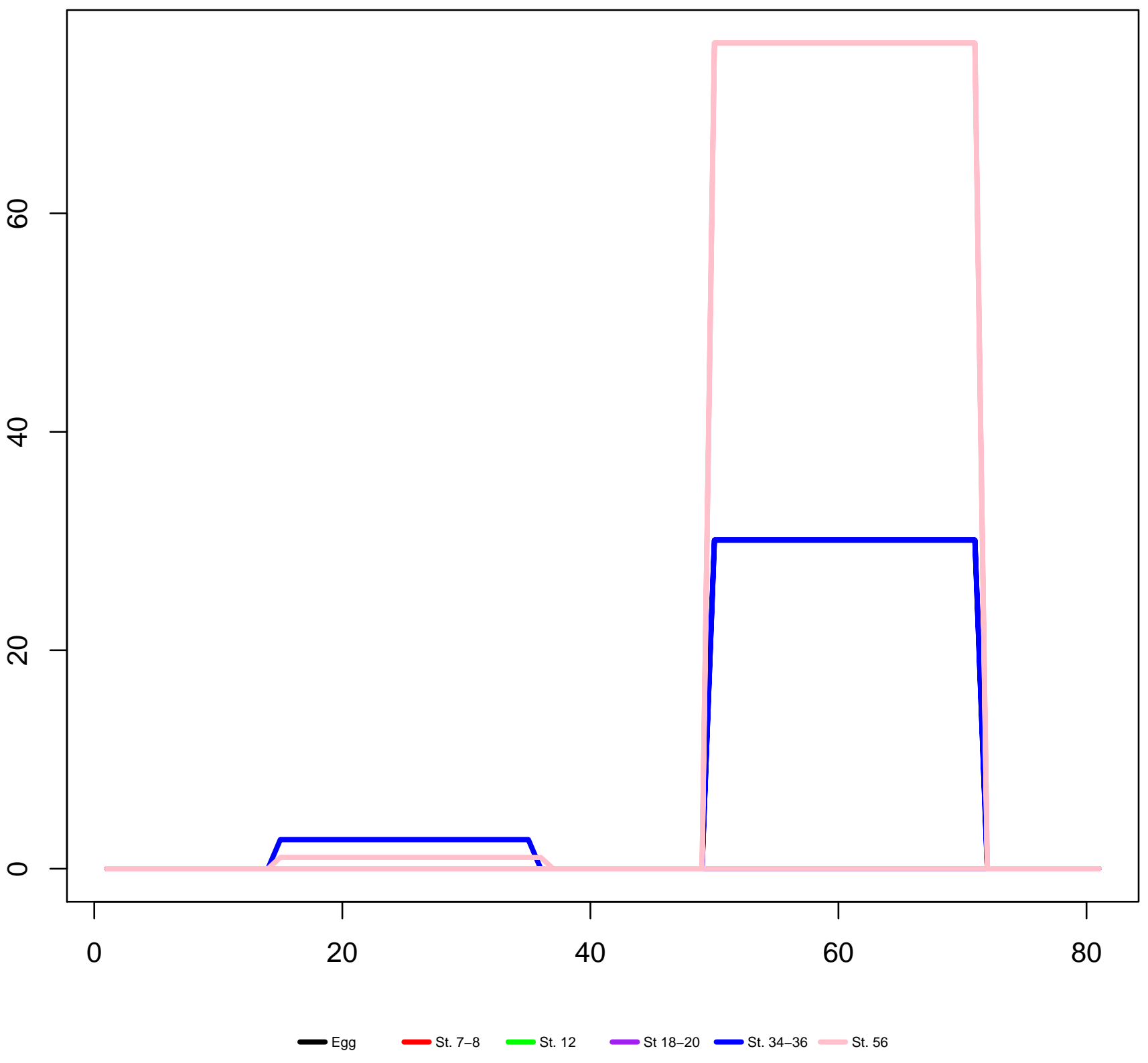


Scaffold44190_146942-147010(-) mir-139

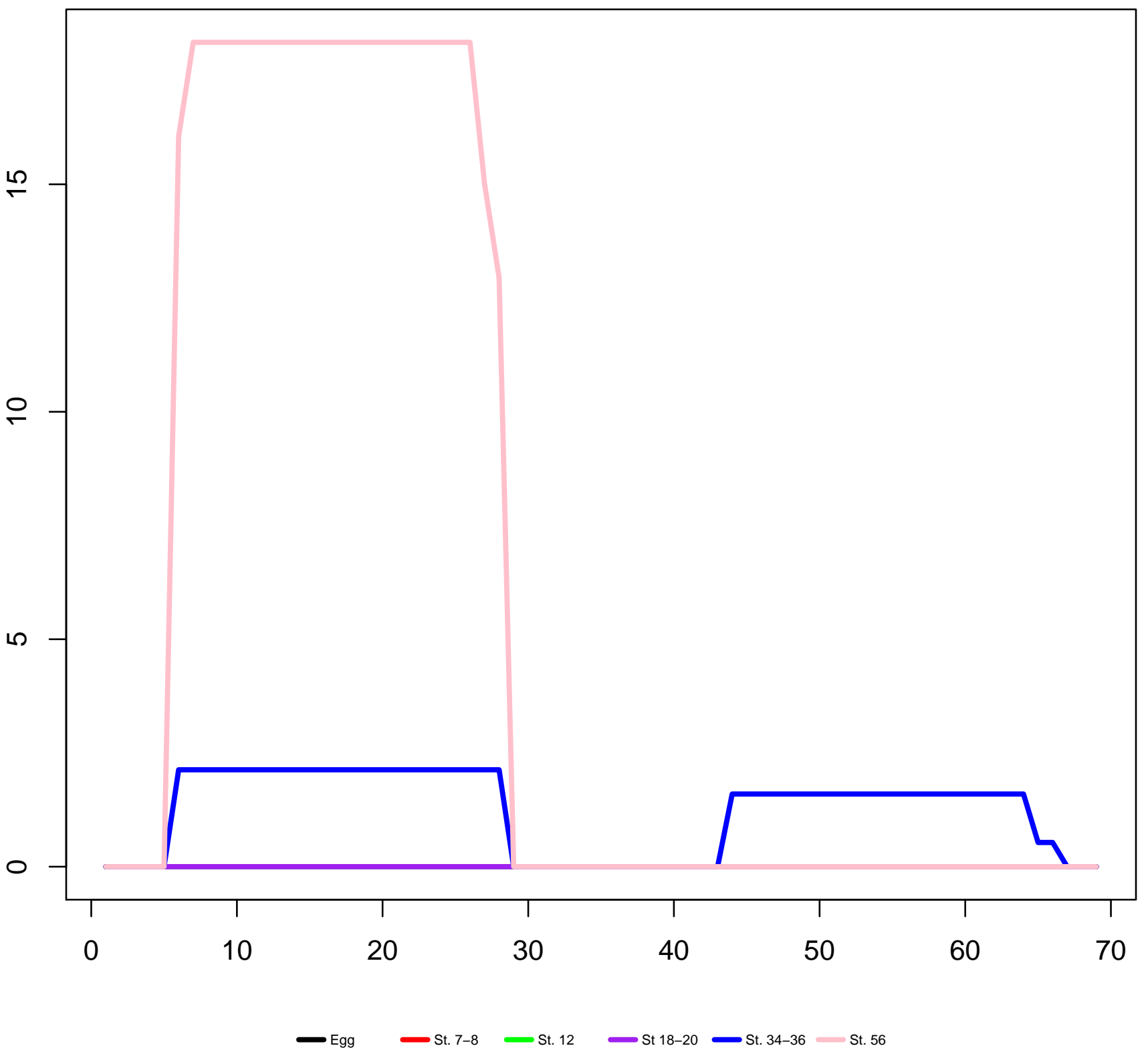


Scaffold44956_126925-126992(-) mir-33a

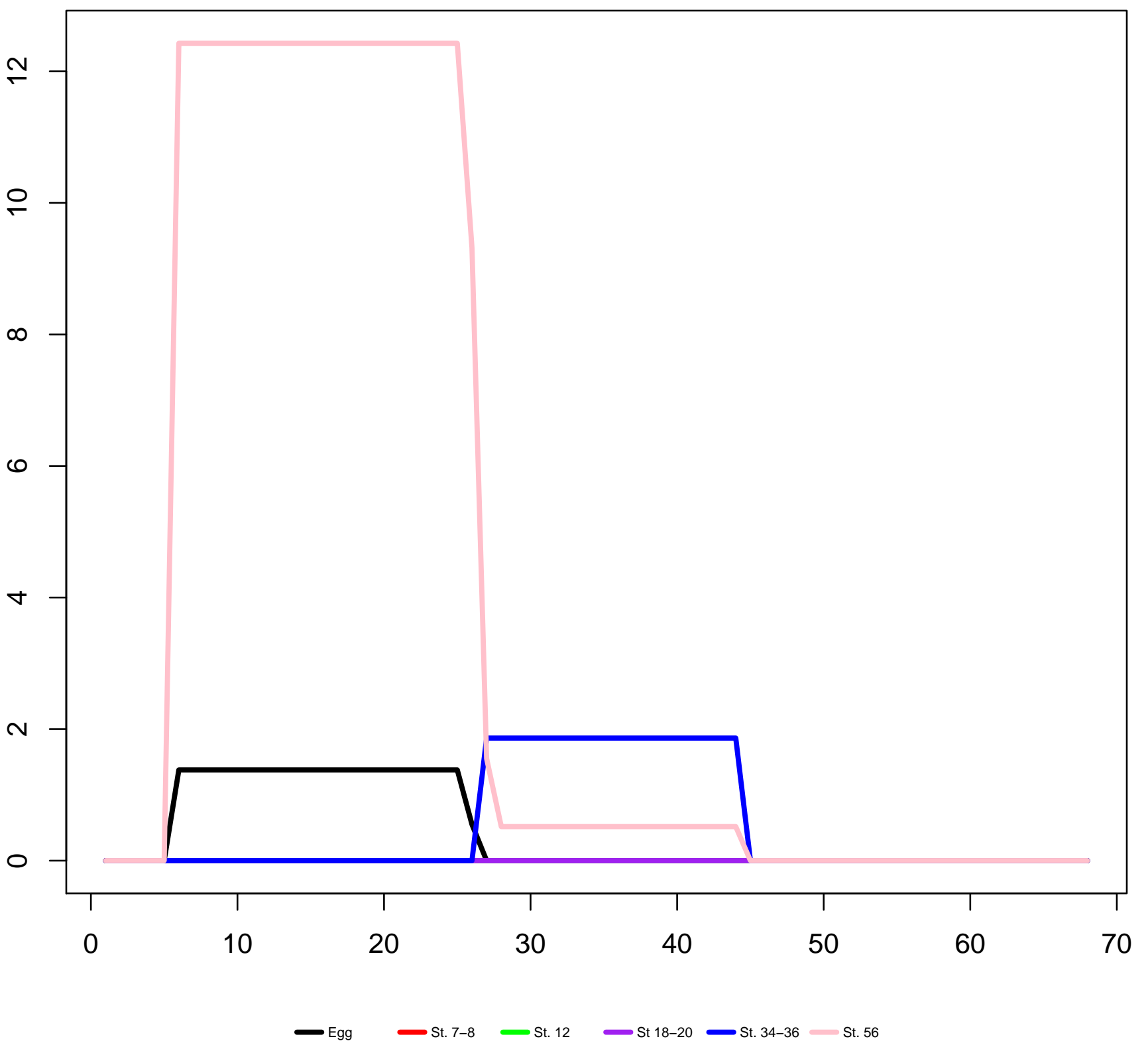


Scaffold45156_692430-692511(+) mir-124a-1

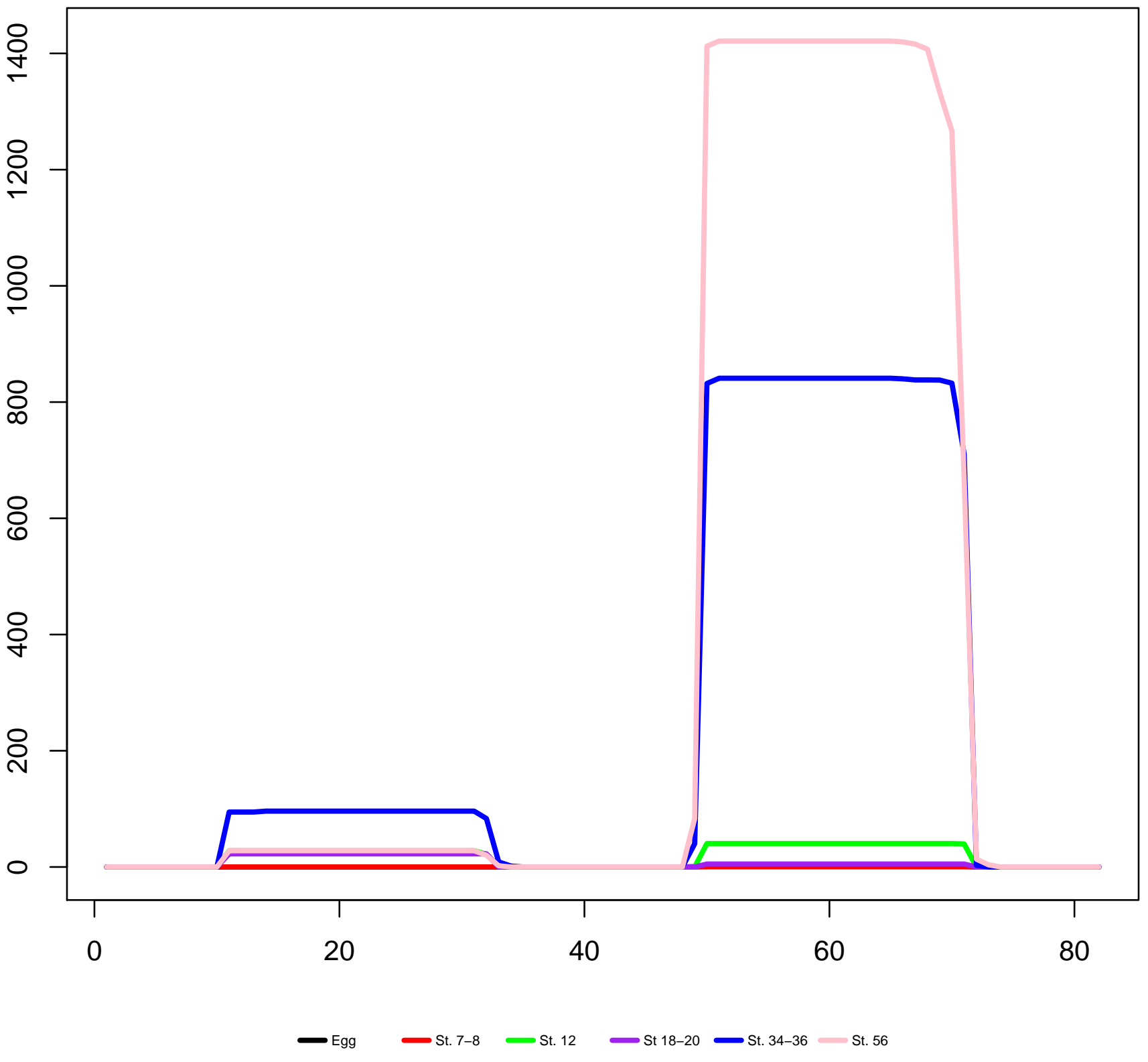


Scaffold4533_544243-544327(-) mir-194-2

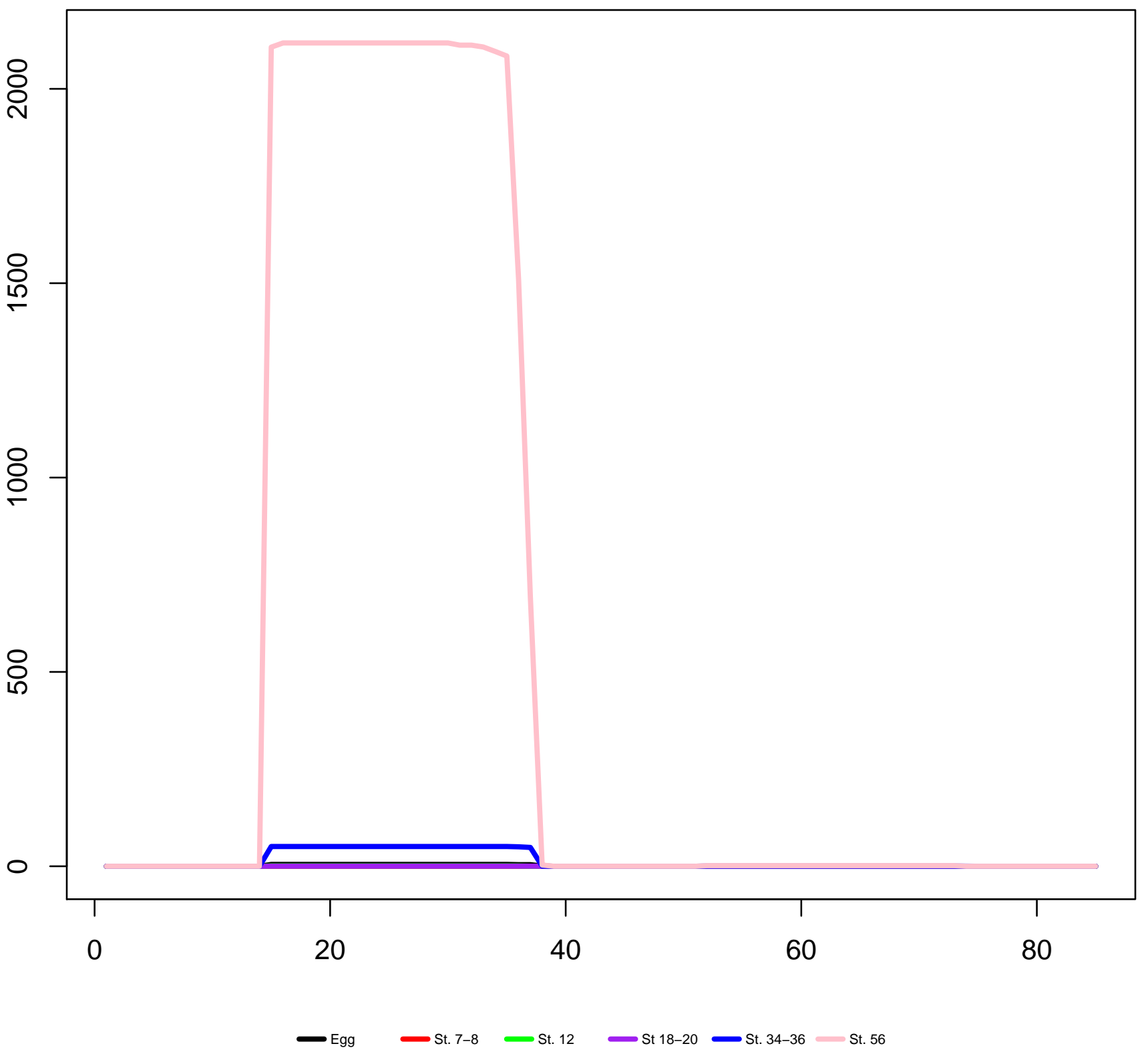


Scaffold45612_637645-637730(-) mir-145

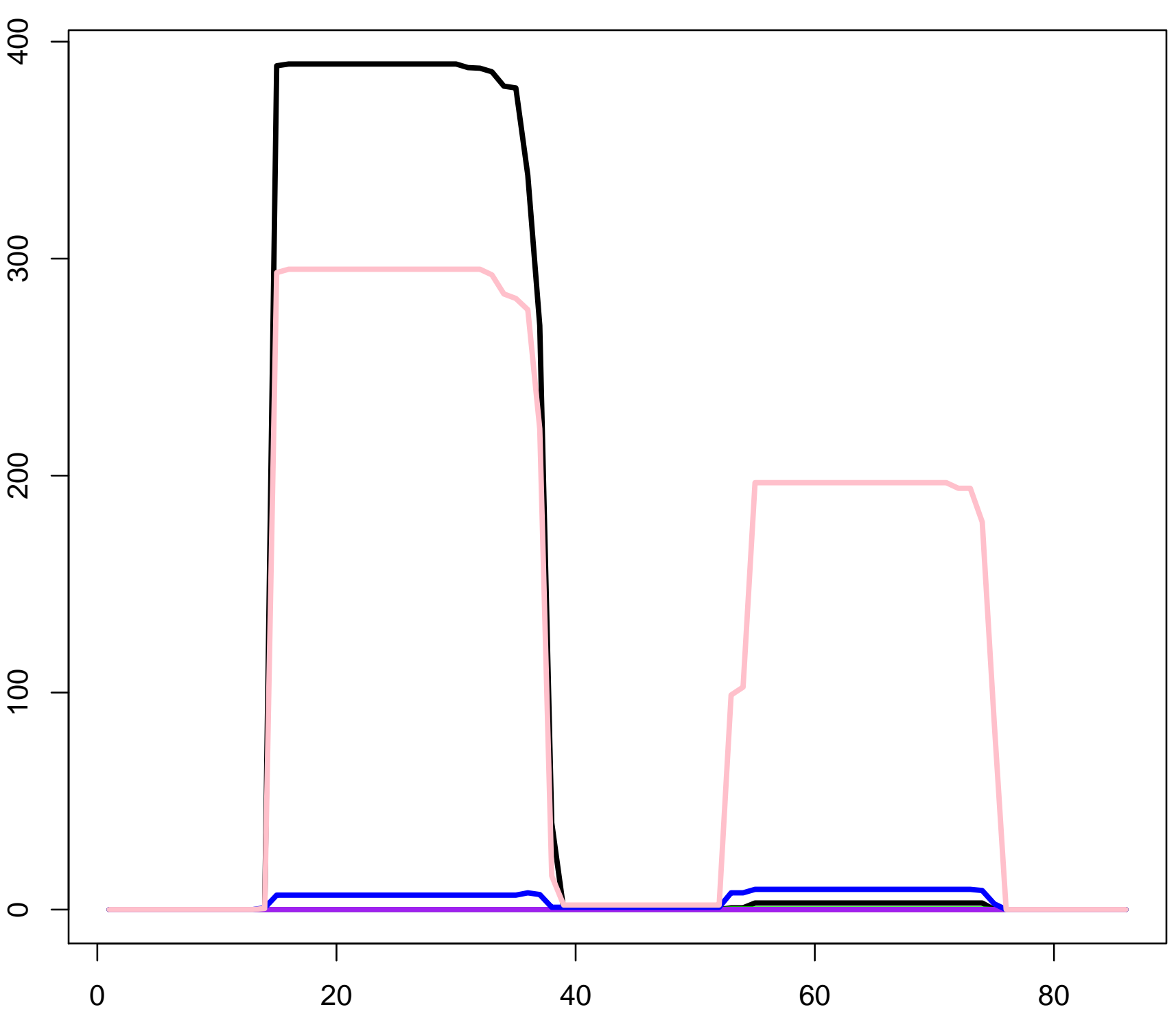

Egg $\longrightarrow$ St. 7-8 $\longrightarrow$ St. $12 \longrightarrow$ St 18-20 St. 34-36 $\longrightarrow$ St. 56 
Scaffold45612_638678-638758(-) mir-143

용

응

ঃ

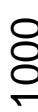


Scaffold457743_33-118(-) mir-218-2

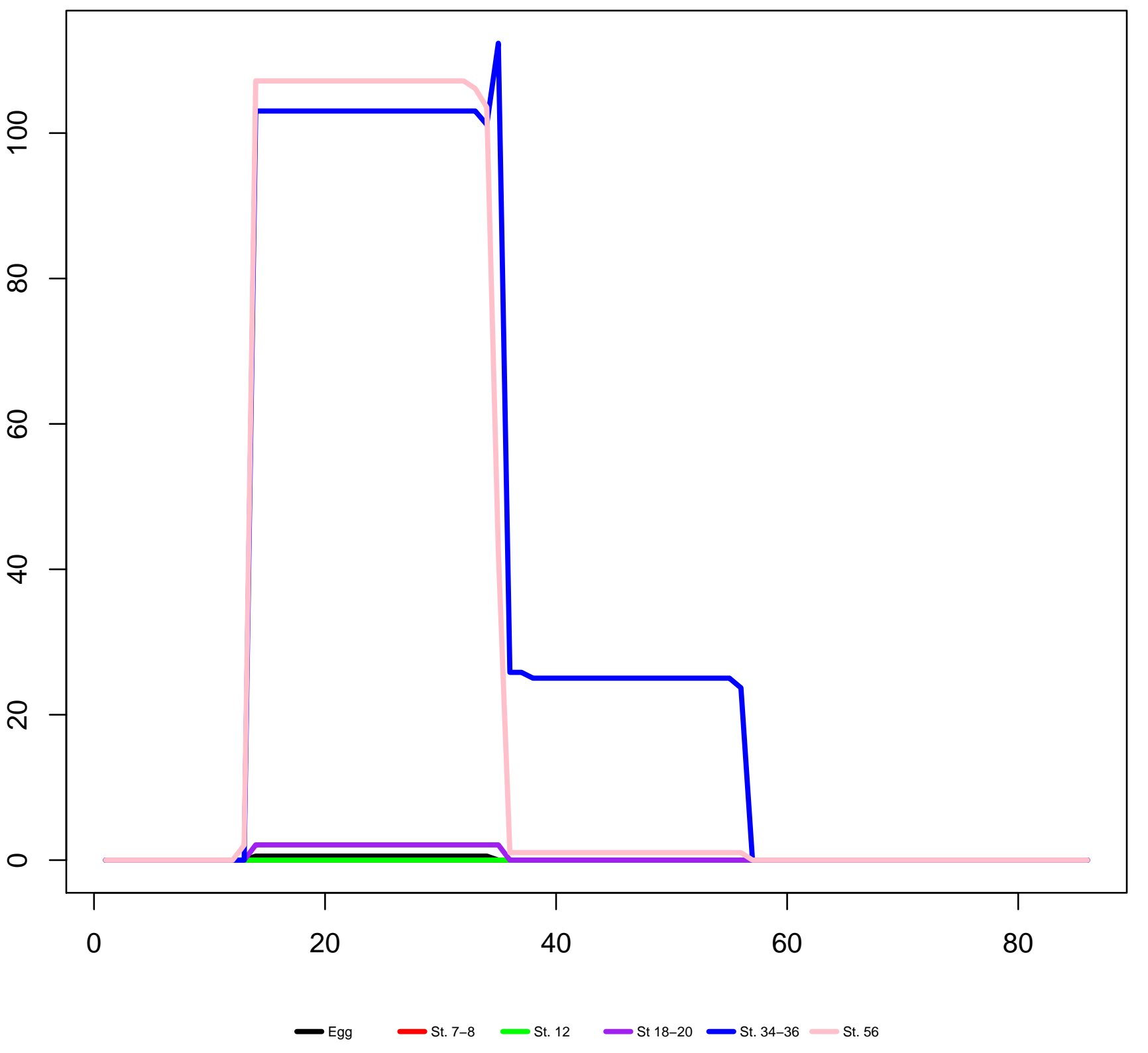


Scaffold462576_1014-1102(-) let-7c-2

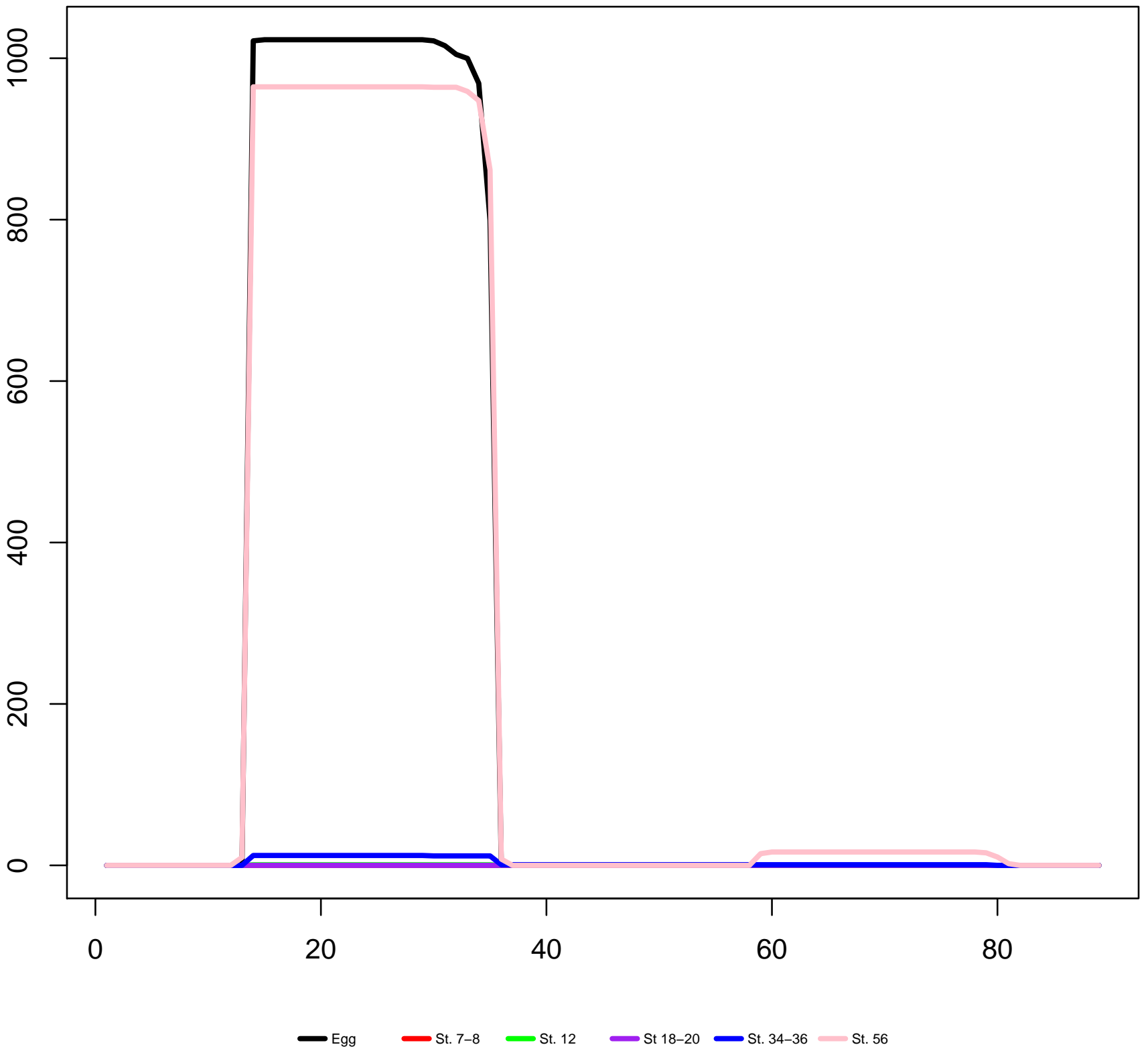


Scaffold46437_1016072-1016182(+) let-7c-1

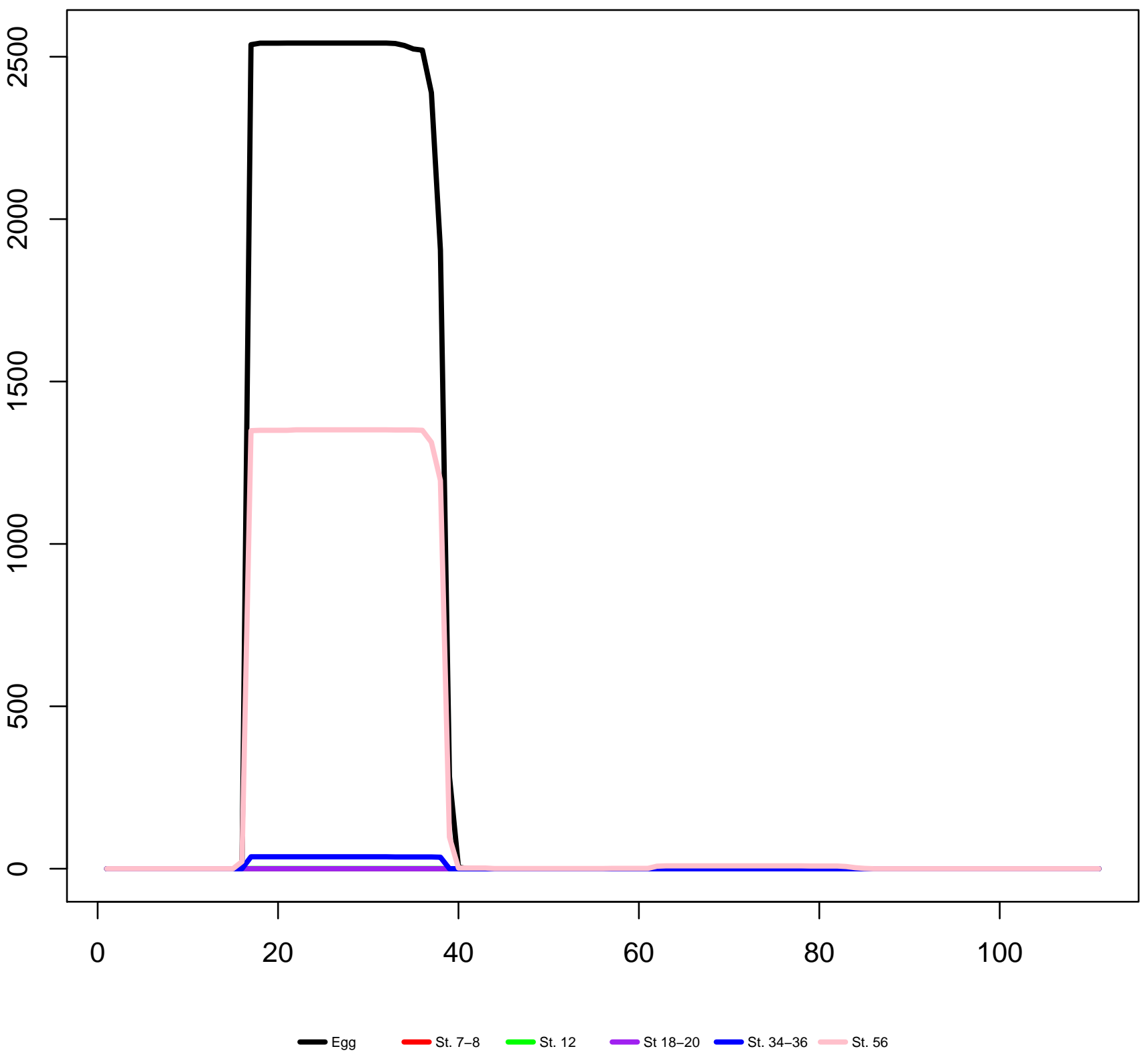


Scaffold47083_1952177-1952277(-) mir-383

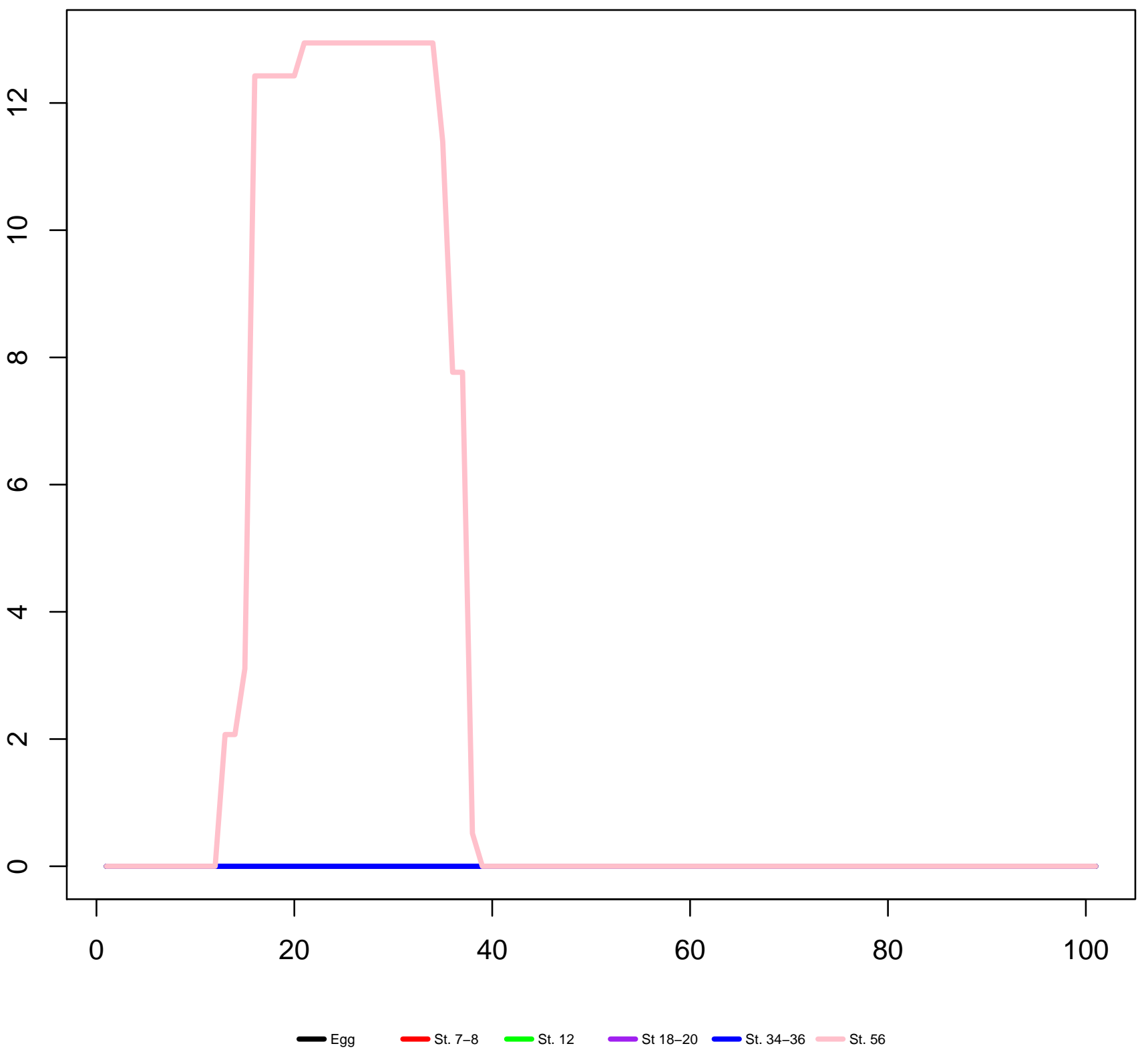


Scaffold48041_909007-909104(-) mir-9-1

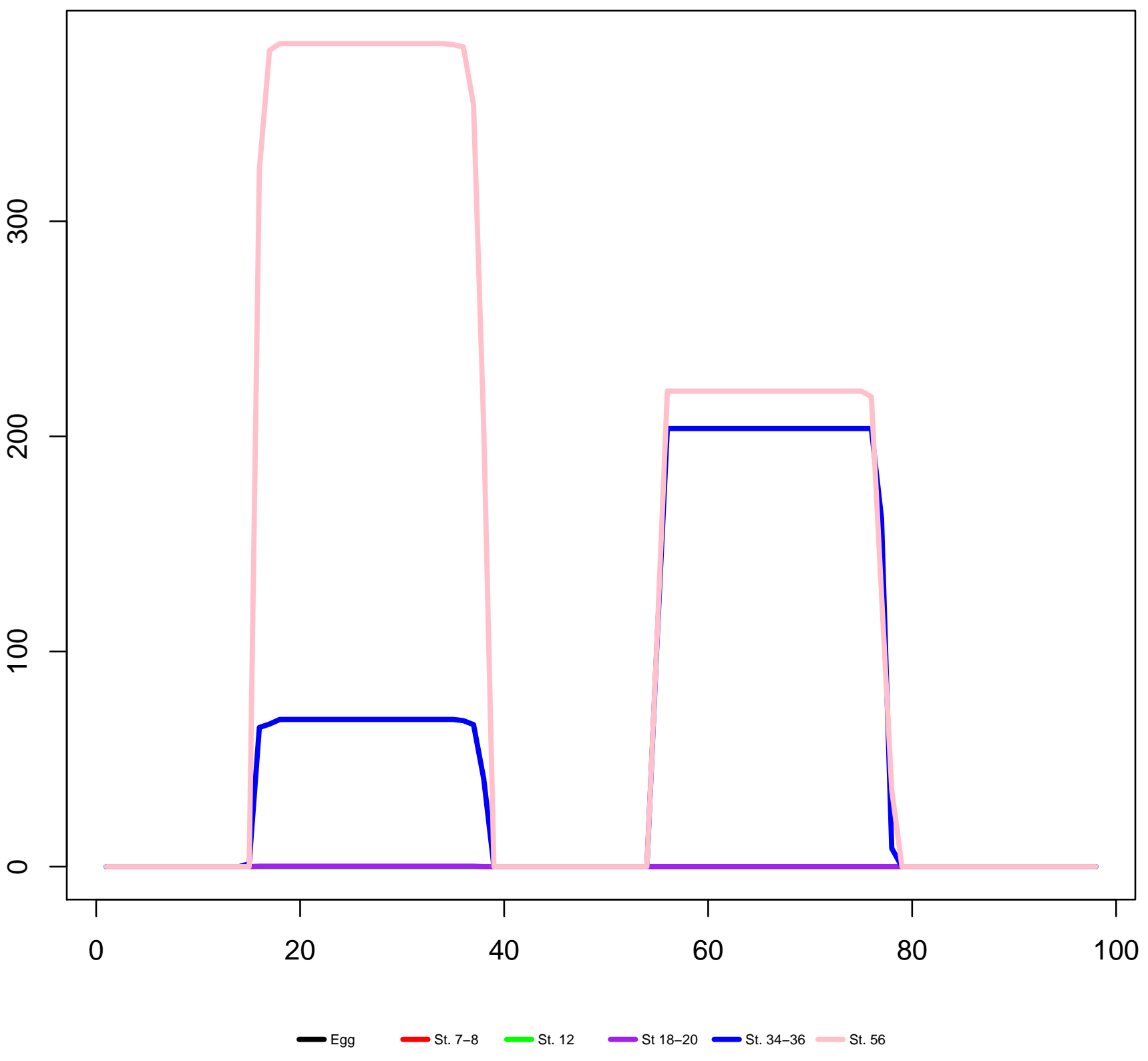


Scaffold48150_316131-316196(+) mir-93a

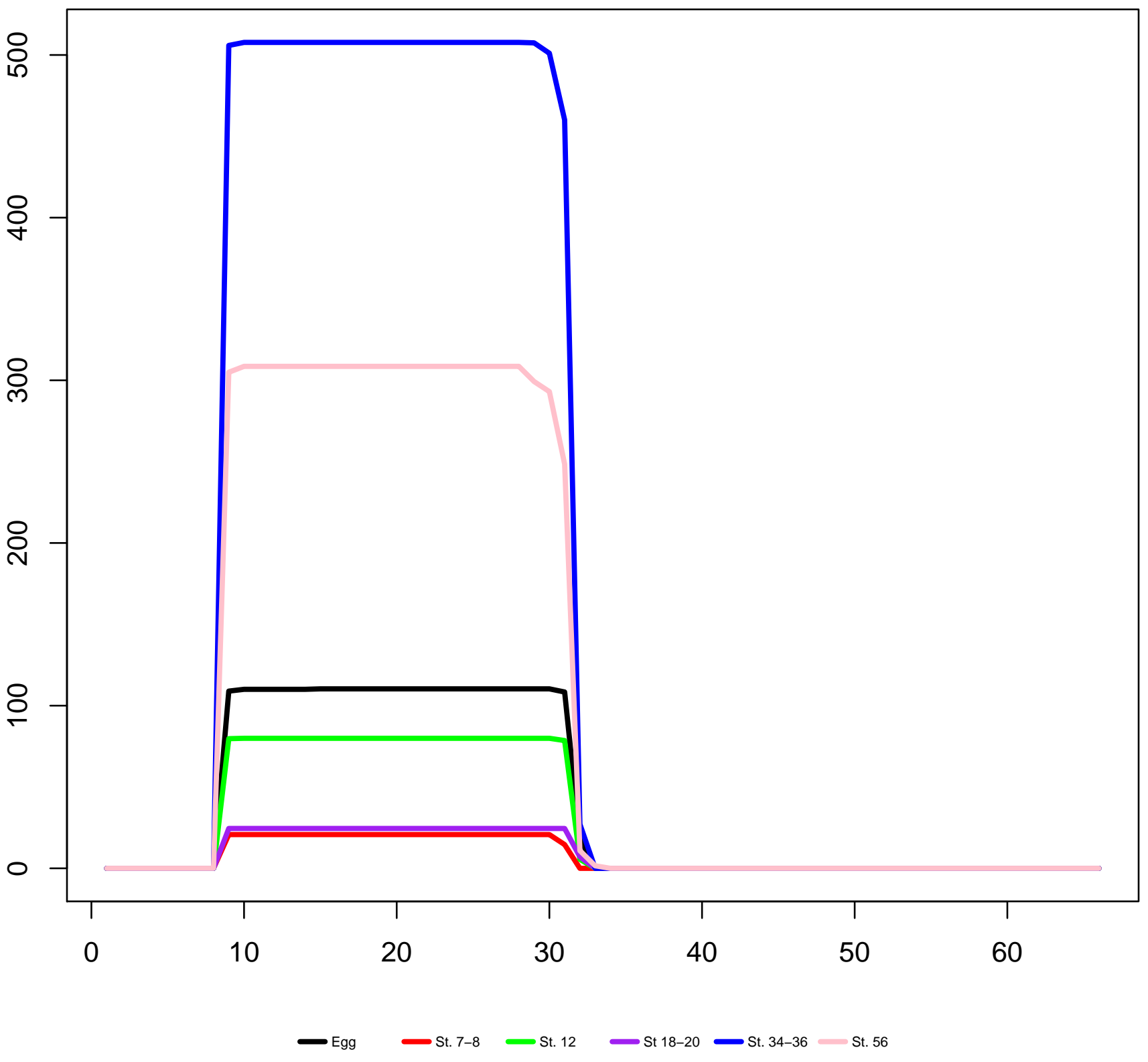


Scaffold4890_1595528-1595624(-) mir-9-1

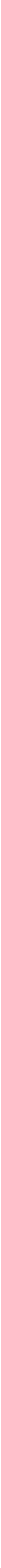


Scaffold513544_15-116(-) mir-21

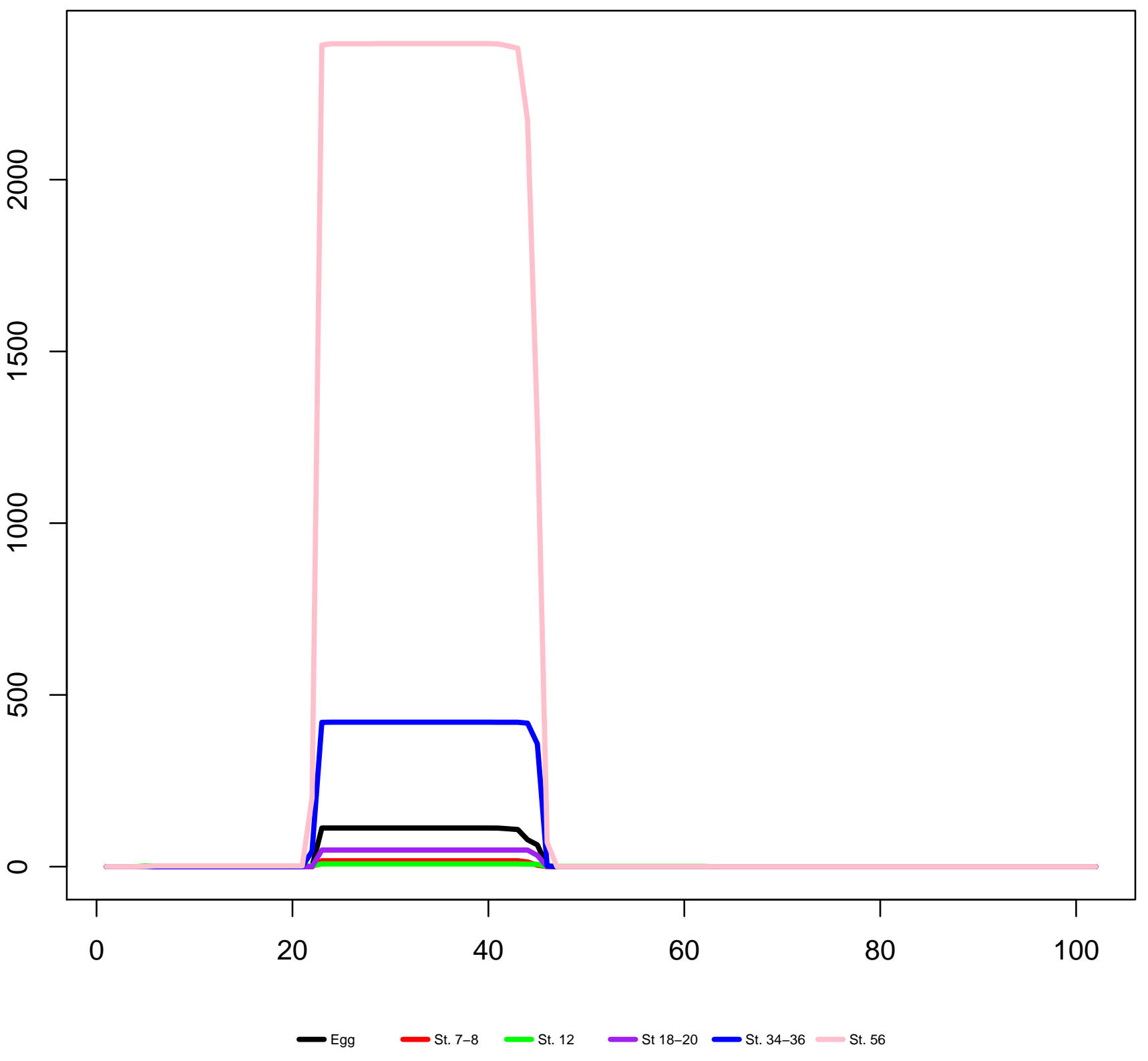


Scaffold514865_2339-2406(-) mir-427

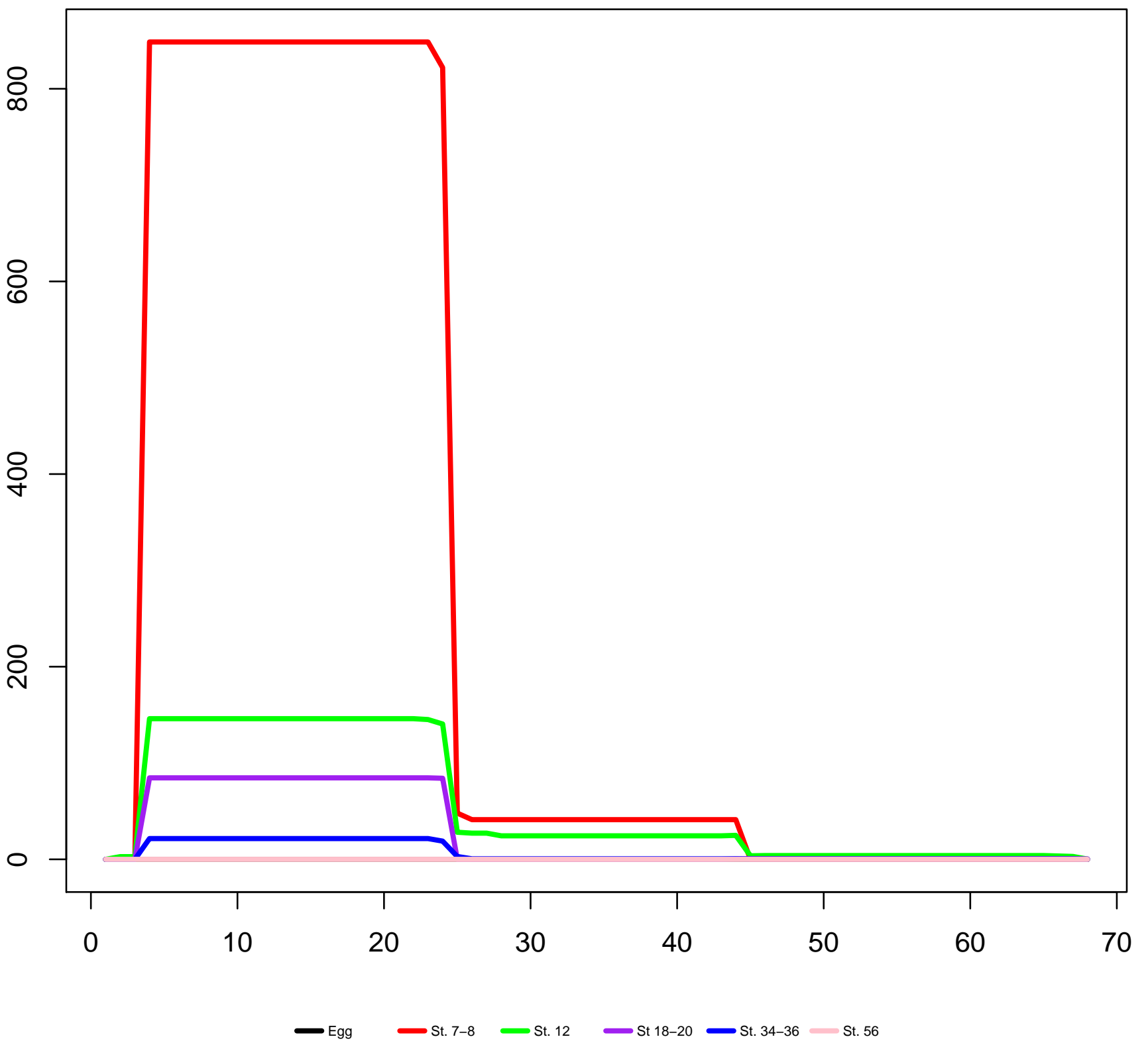


Scaffold51656_202865-202991(+) mir-7-2

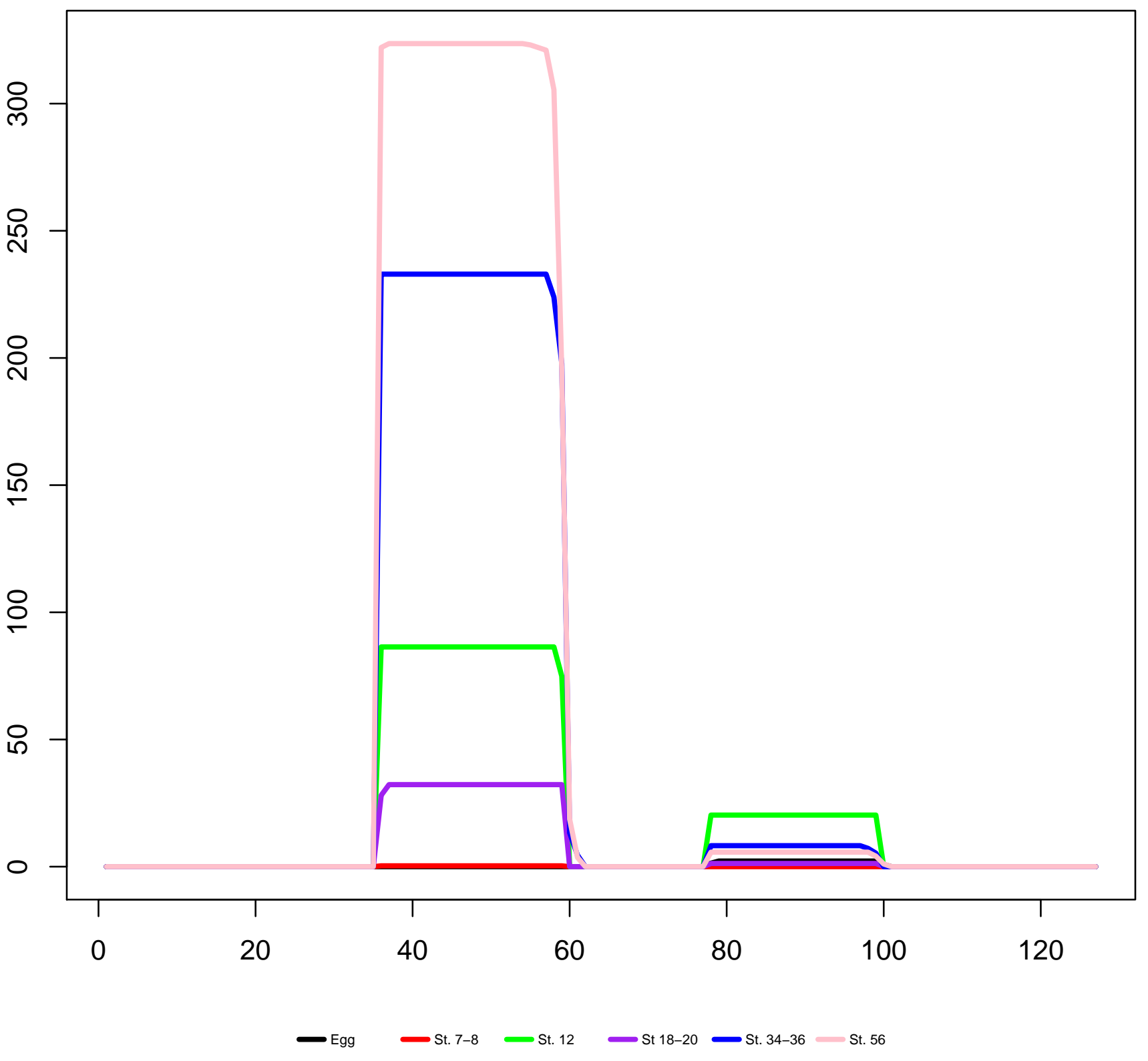




\section{Scaffold52961_726790-726866(+) mir-130c}

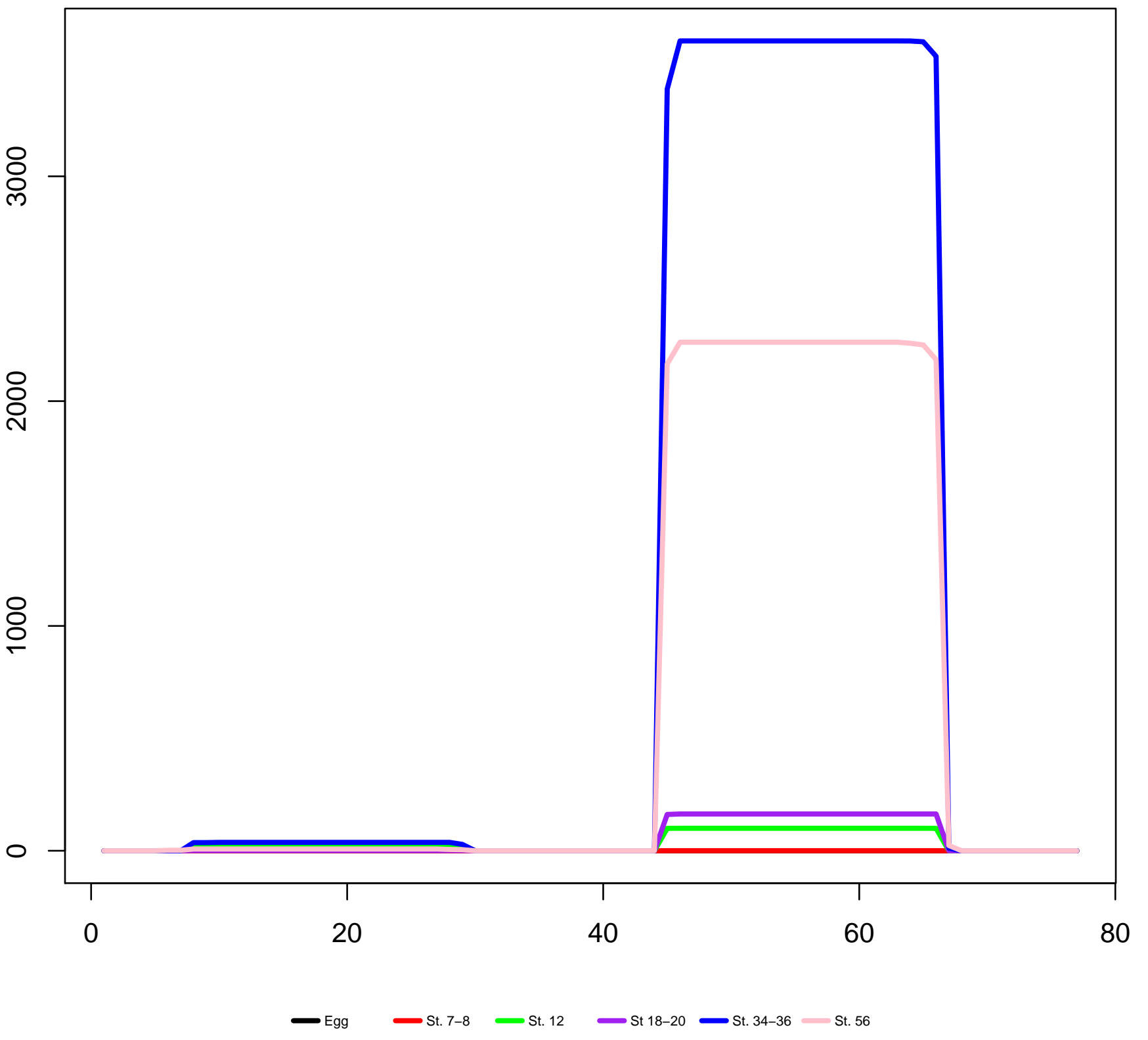


Scaffold52961_732952-733032(+) mir-454

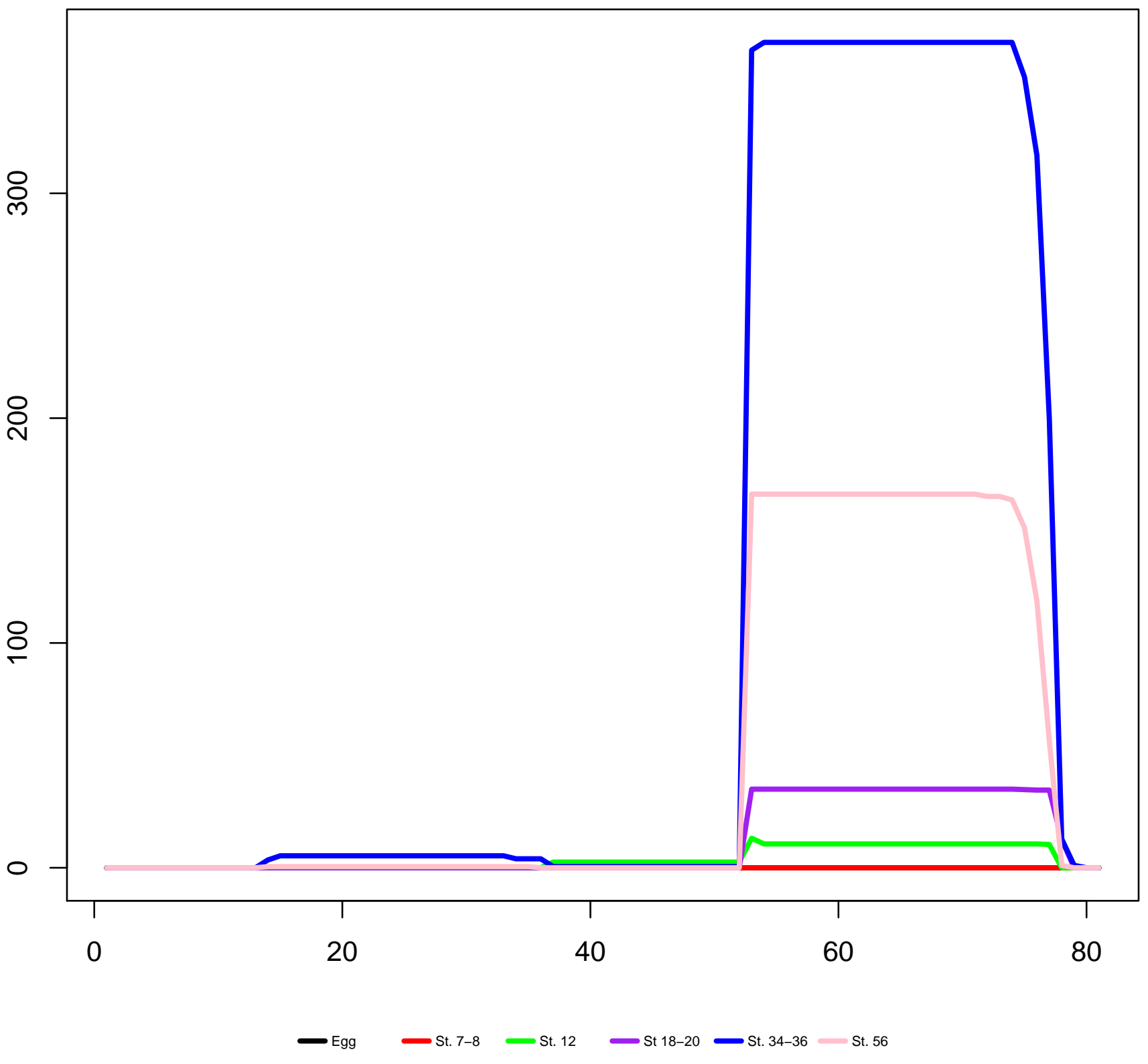


Scaffold55555_5519-5586(-) mir-427

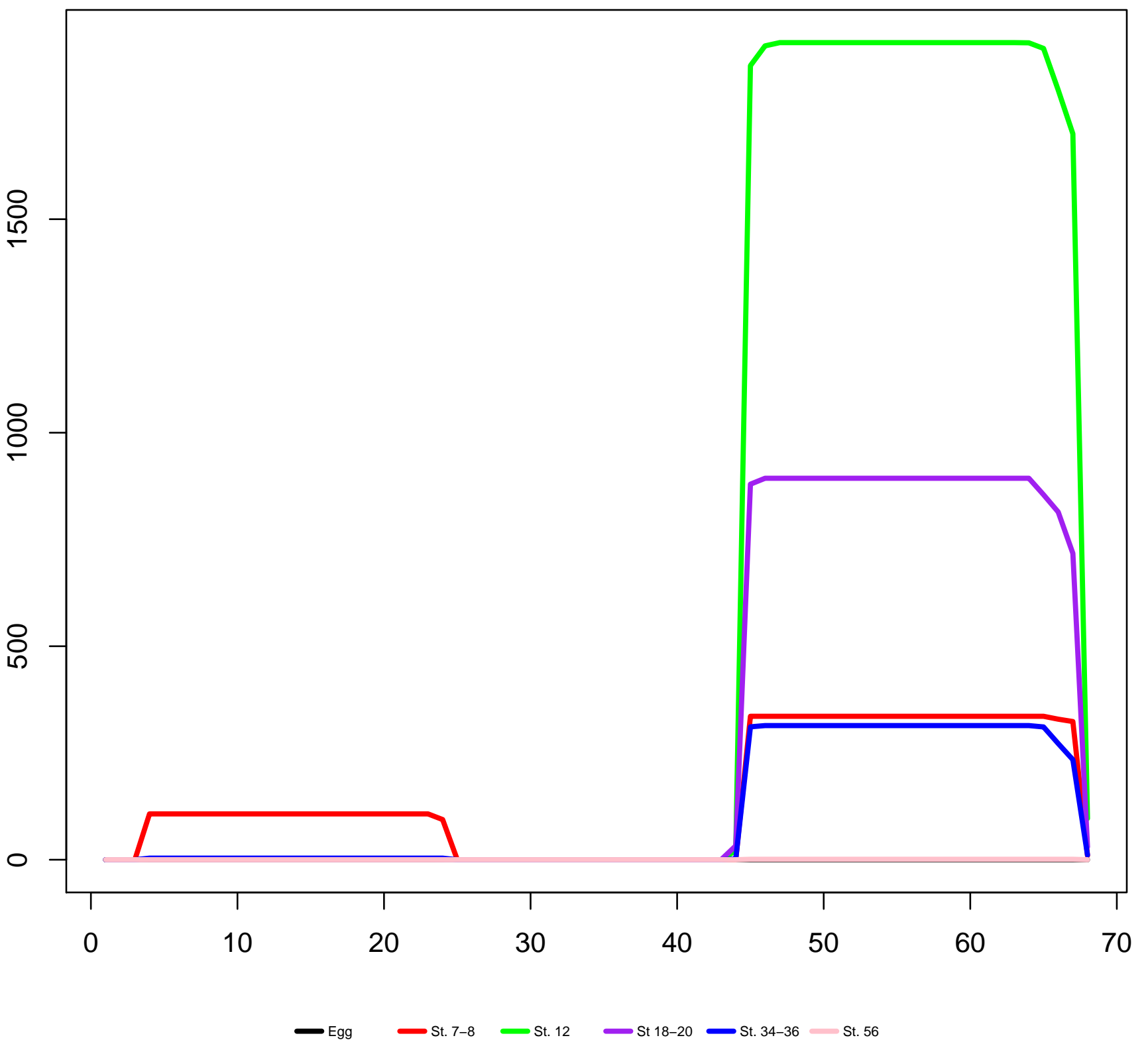


Scaffold58544_148345-148421(-) mir-30b

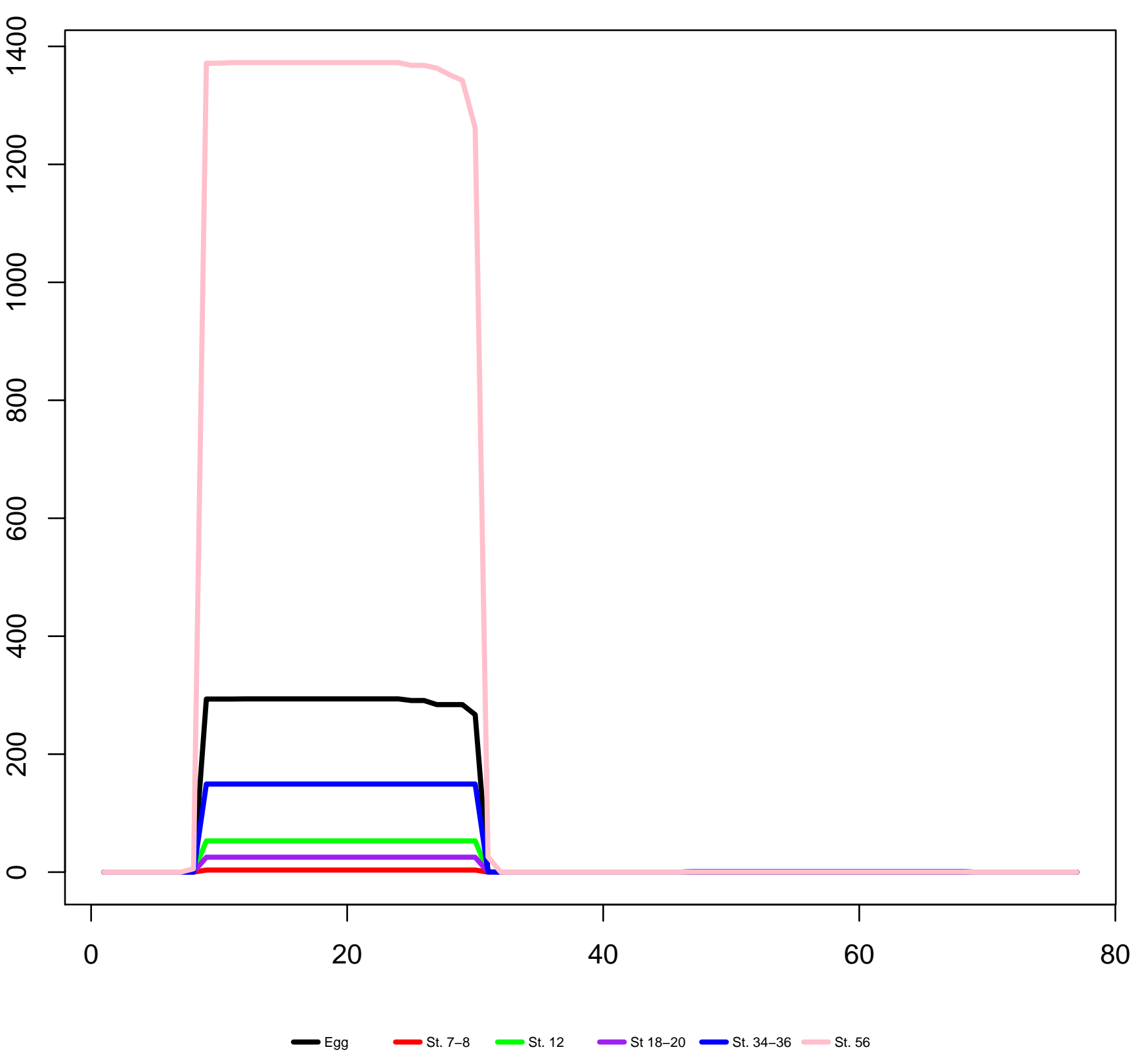


Scaffold58544_151301-151381(-) mir-30d

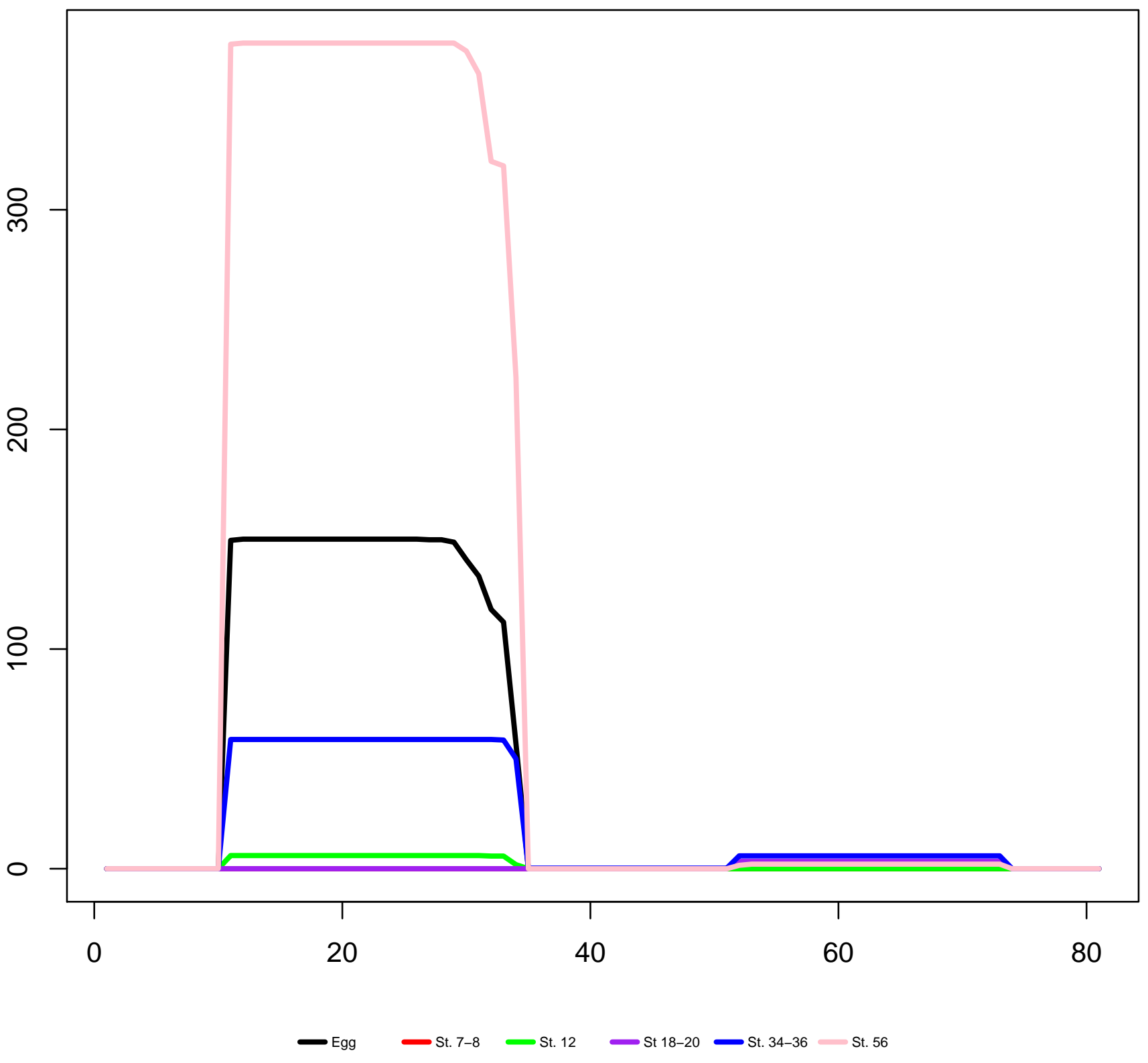


Scaffold5856_717961-718031(+) mir-338

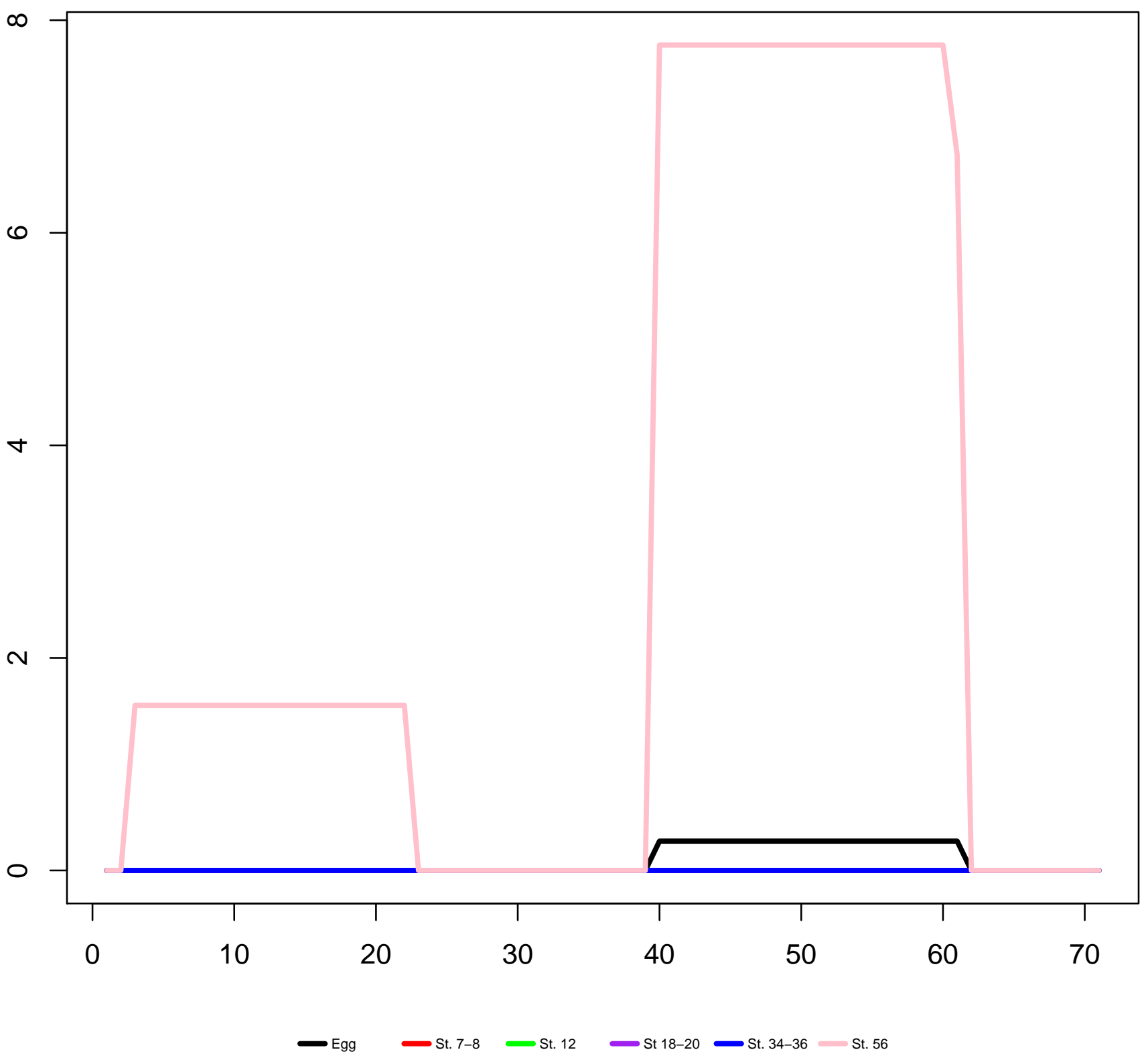


Scaffold61759_728207-728273(-) mir-26-2

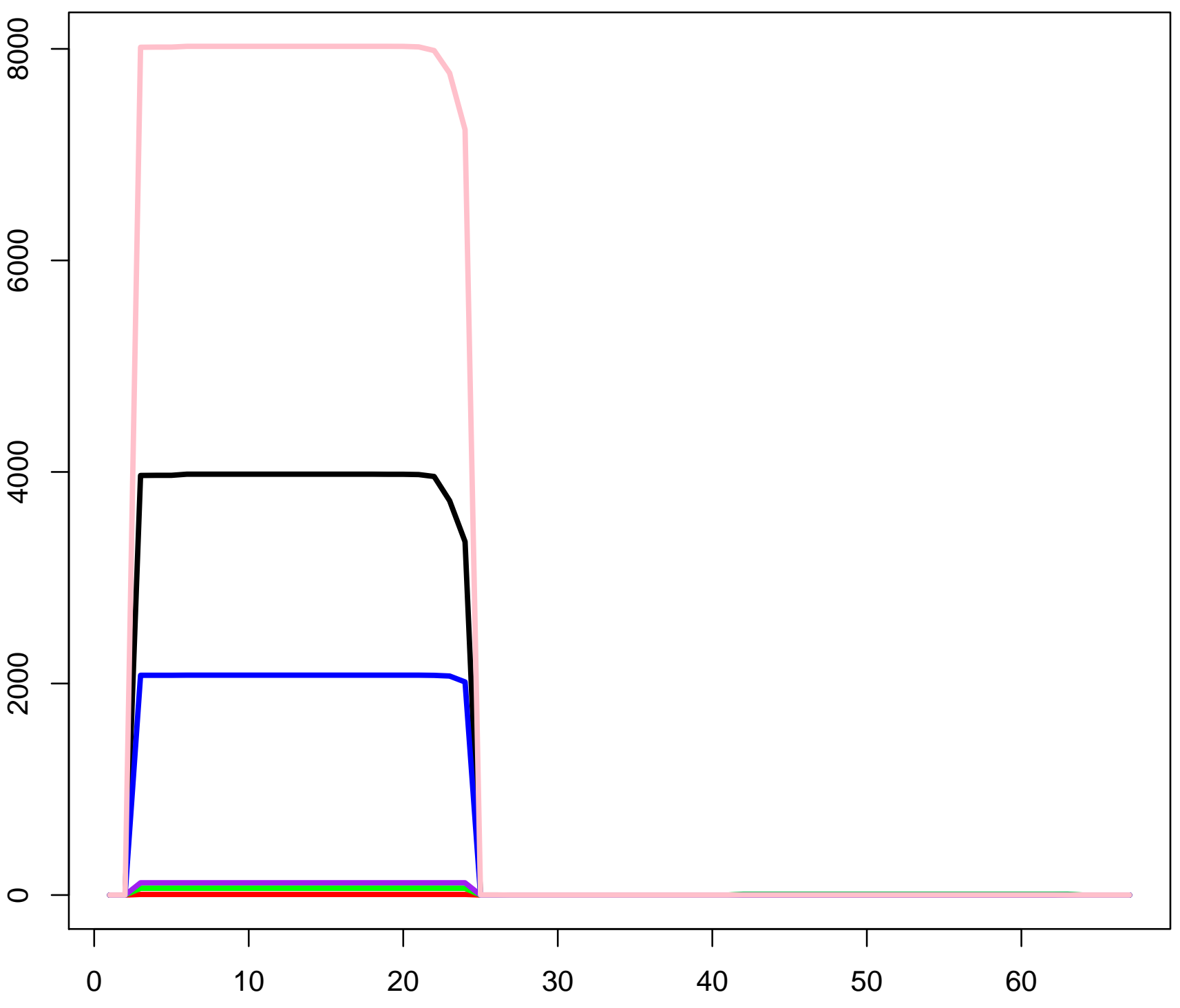


Scaffold62369_1076365-1076450(-) mir-145

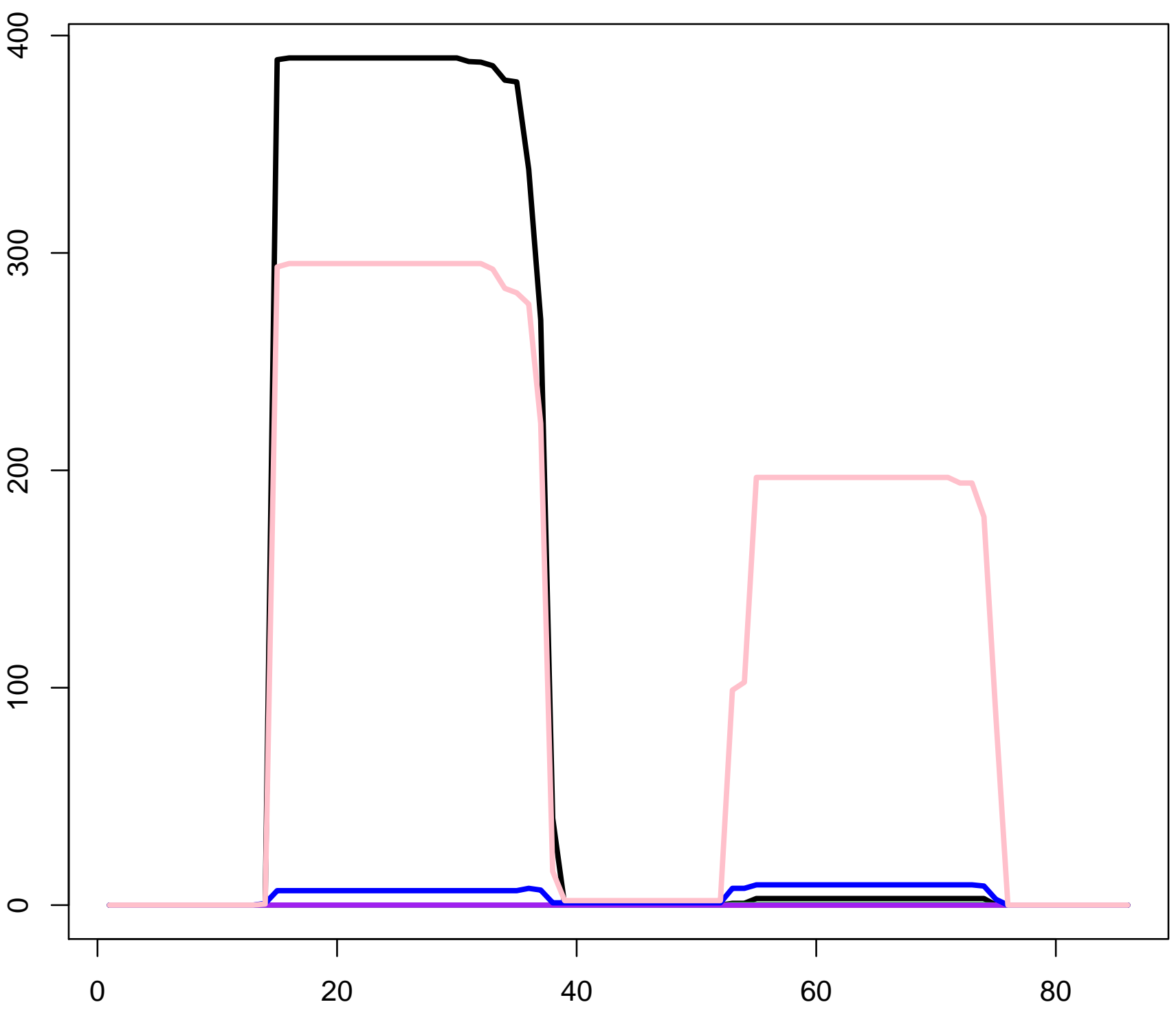

$\longrightarrow$ Egg 2 St. 7-8 St. $12 \rightleftharpoons$ St 18-20 St. 34-36 $\rightleftharpoons$ St. 56 
Scaffold62369_1077310-1077392(-) mir-143

ᄋㅇㅇ

응

응

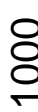


Scaffold62844_195649-195741(+) mir-455

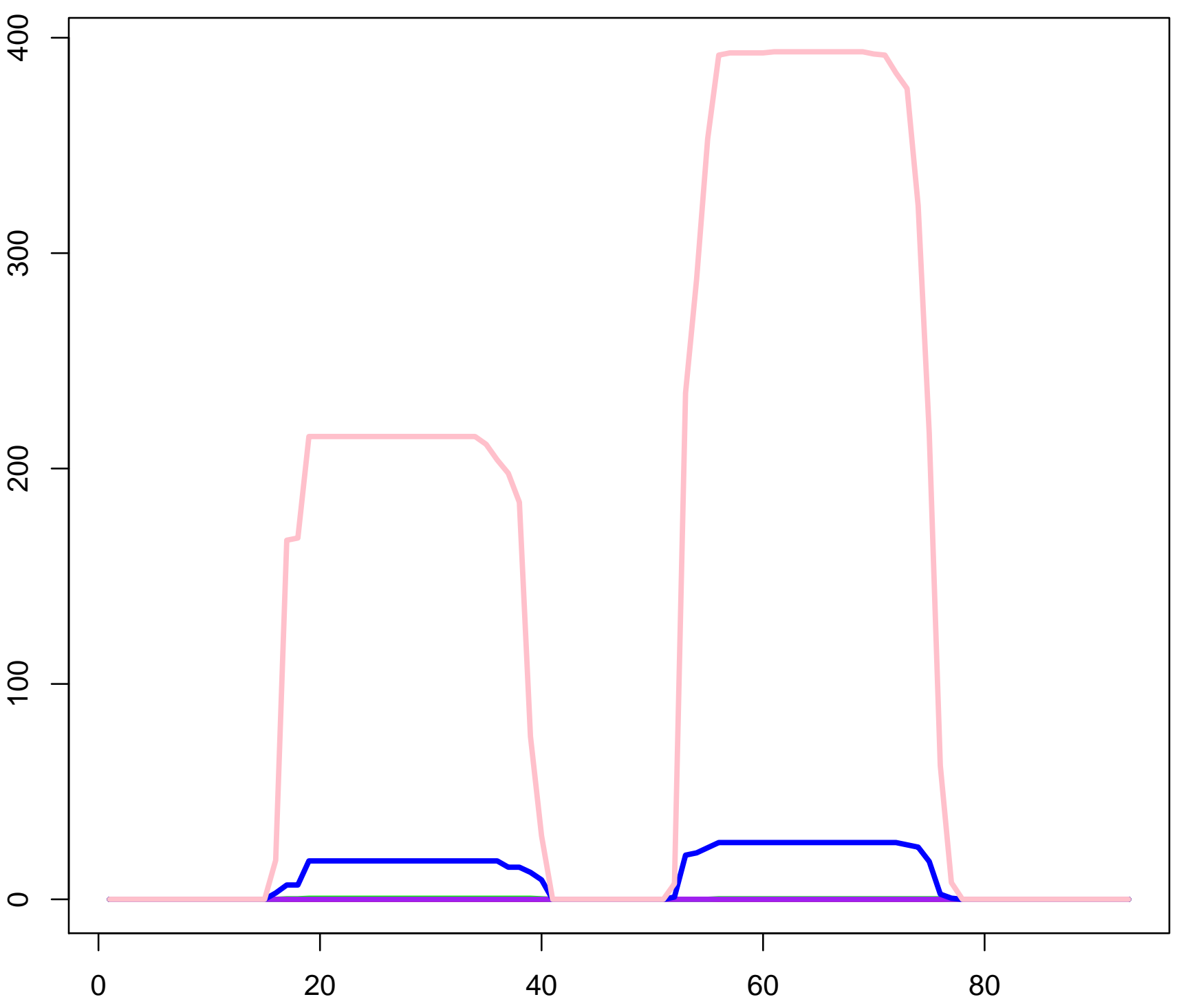

Egg $\longrightarrow$ St. 7-8 $\longrightarrow$ St. $12 \longrightarrow$ St 18-20 St. 34-36 St. 56 
Scaffold65025_78793-78871(-) mir-26

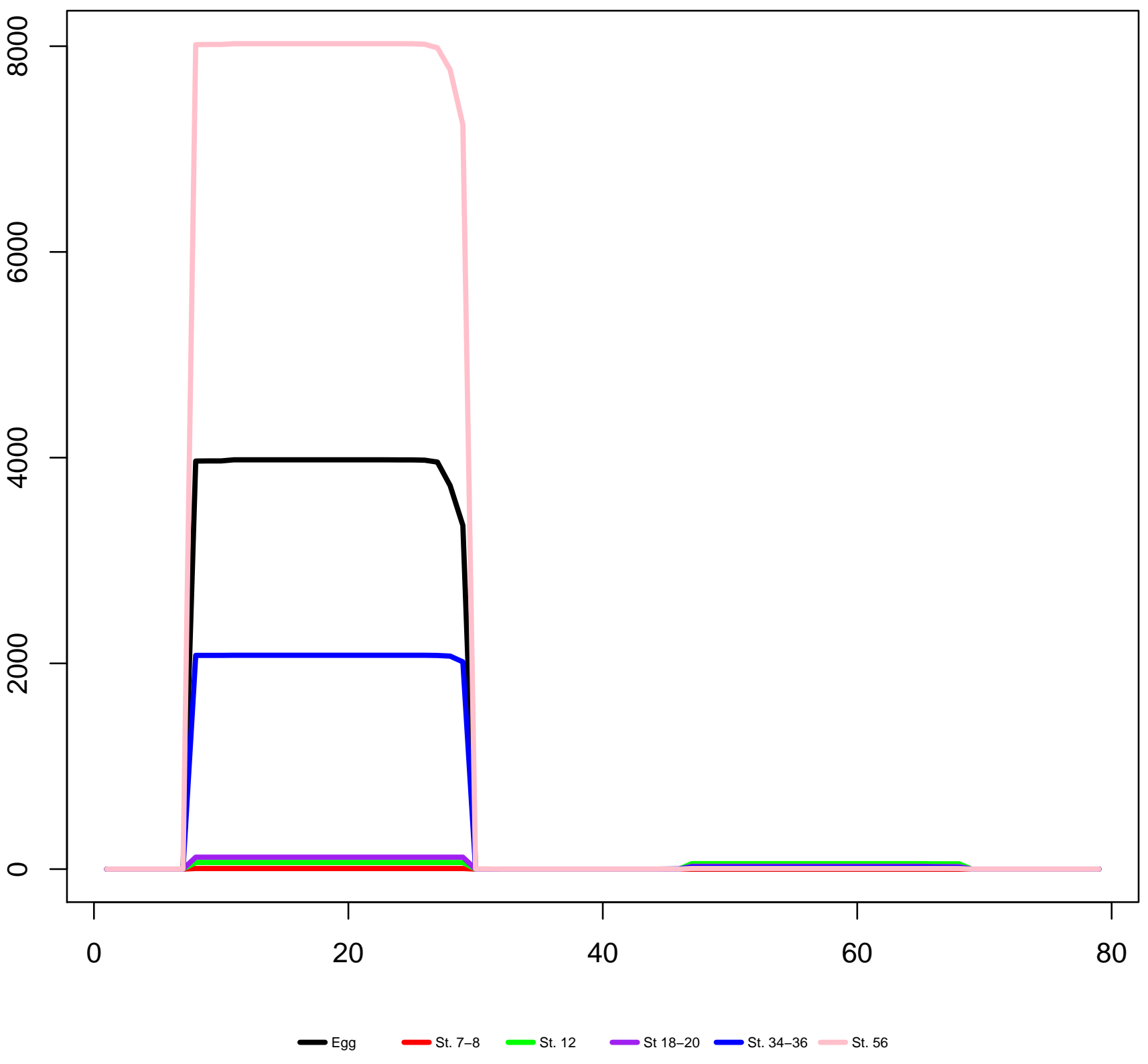


Scaffold6528_63517-63602(+) let-7a

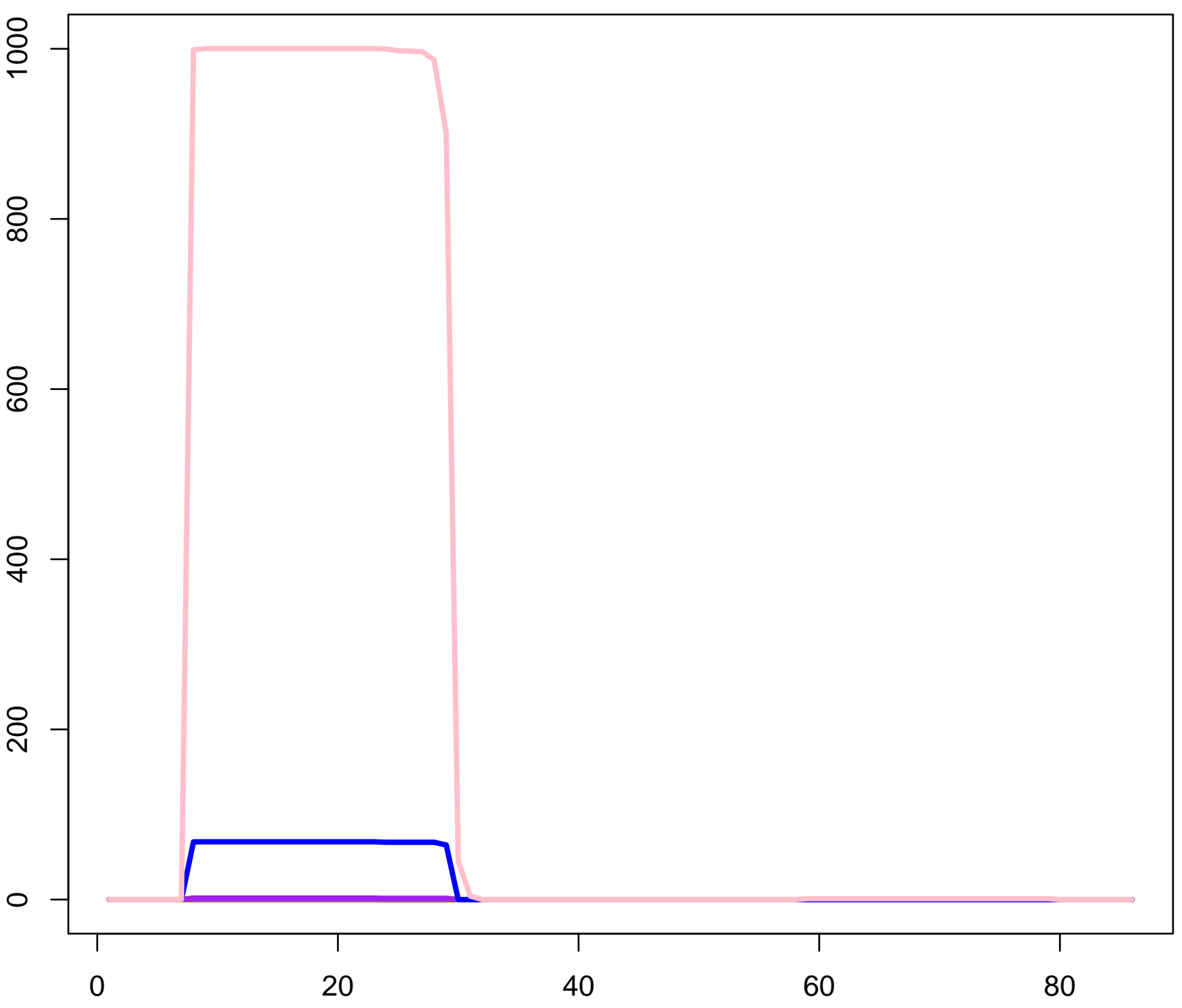


Scaffold67186_145143-145241(-) mir-125b-1

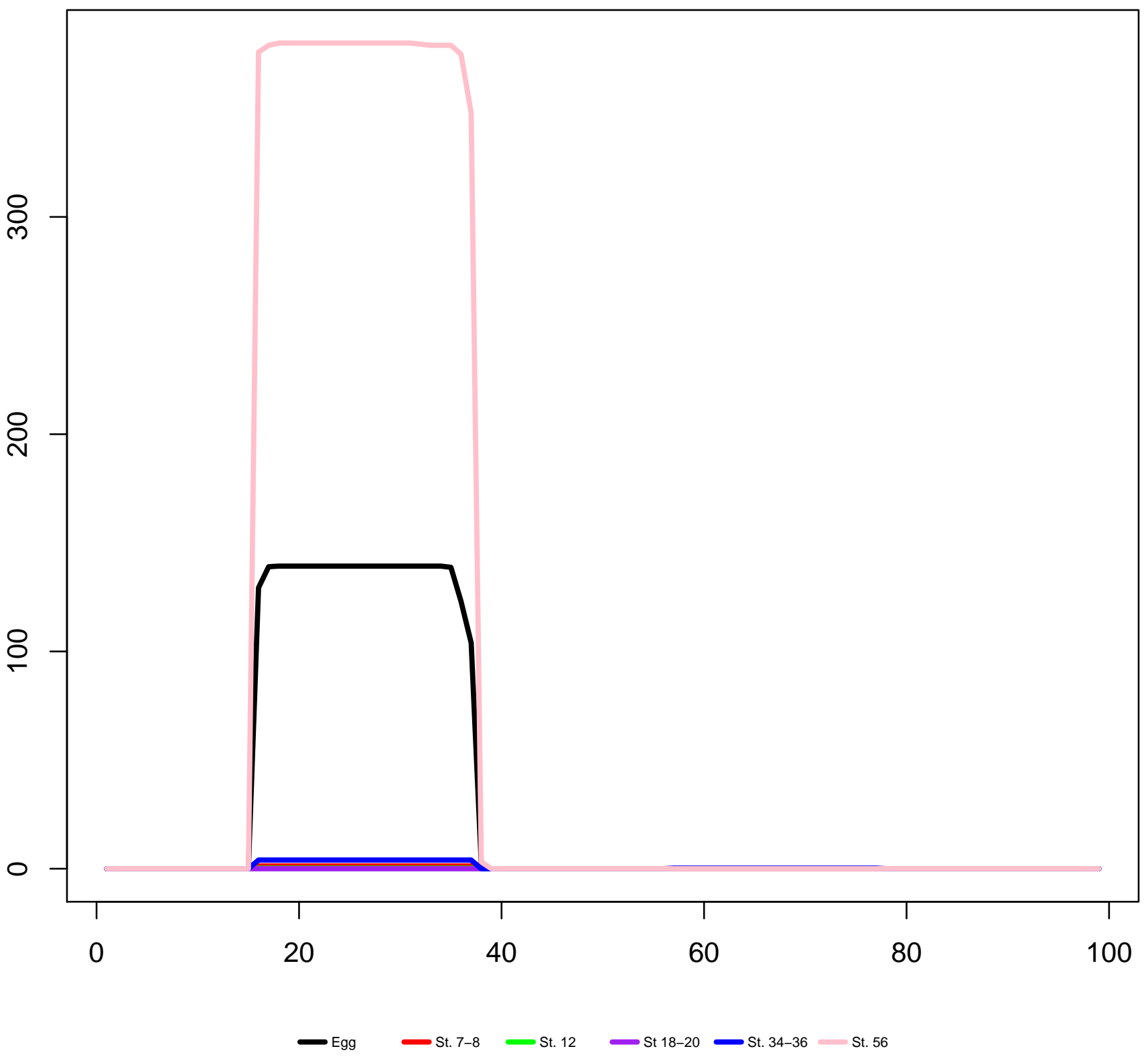


Scaffold6719_612909-612988(+) mir-16b

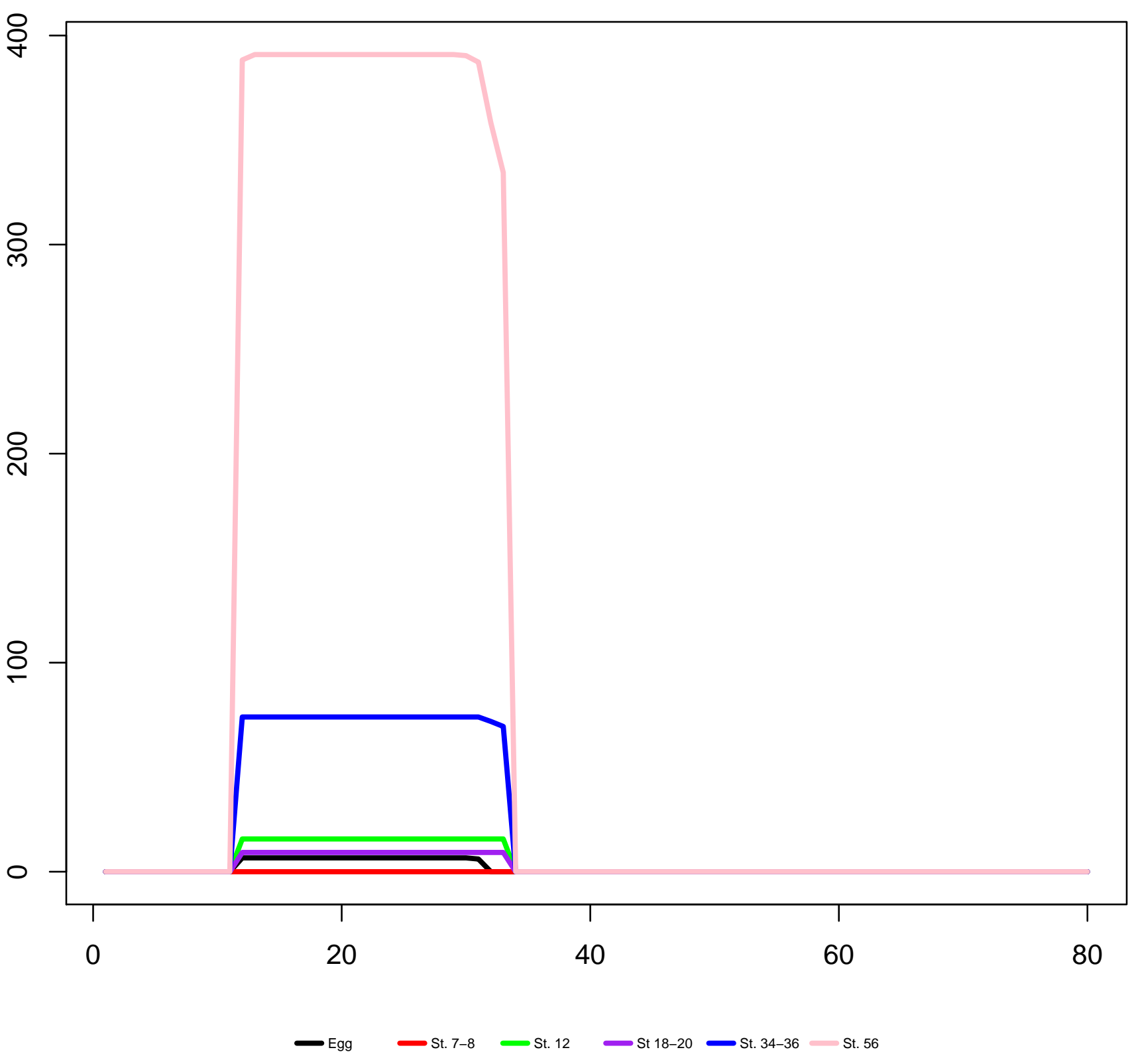


Scaffold67649_101498-101566(+) mir-26-2

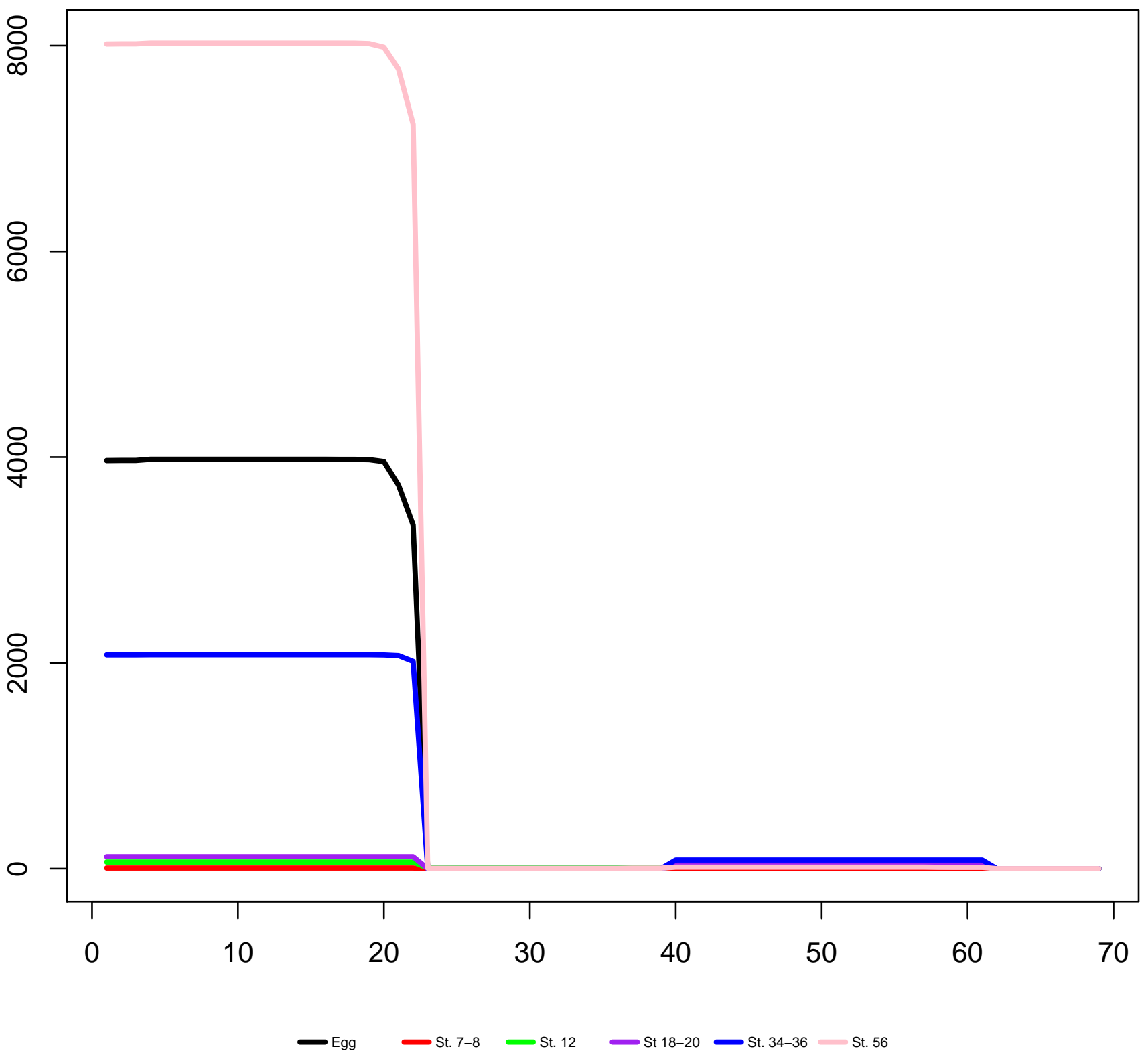




\section{Scaffold6801_52439-52531(+) mir-10a}

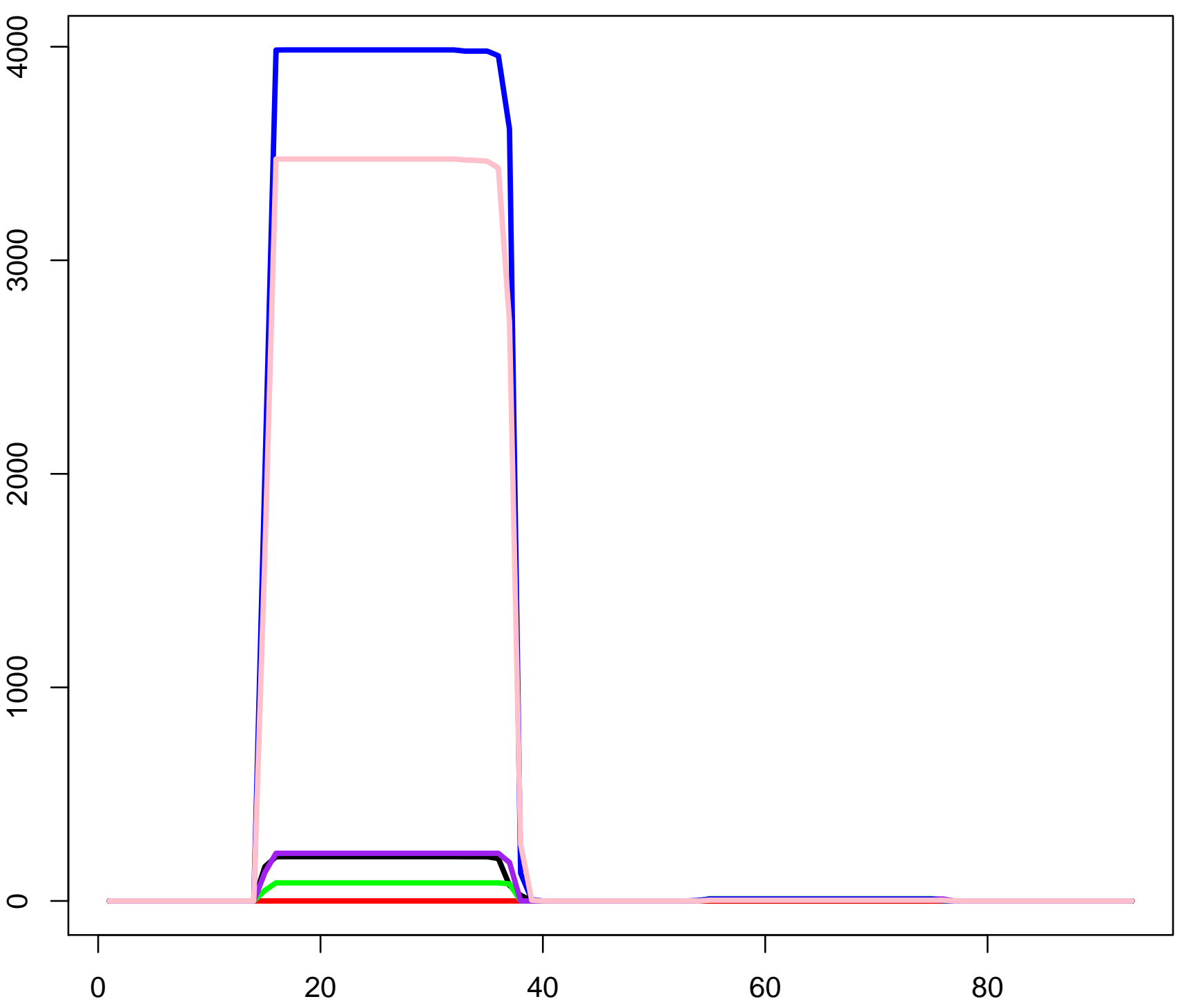


Scaffold68058_3-96(+) mir-101-1

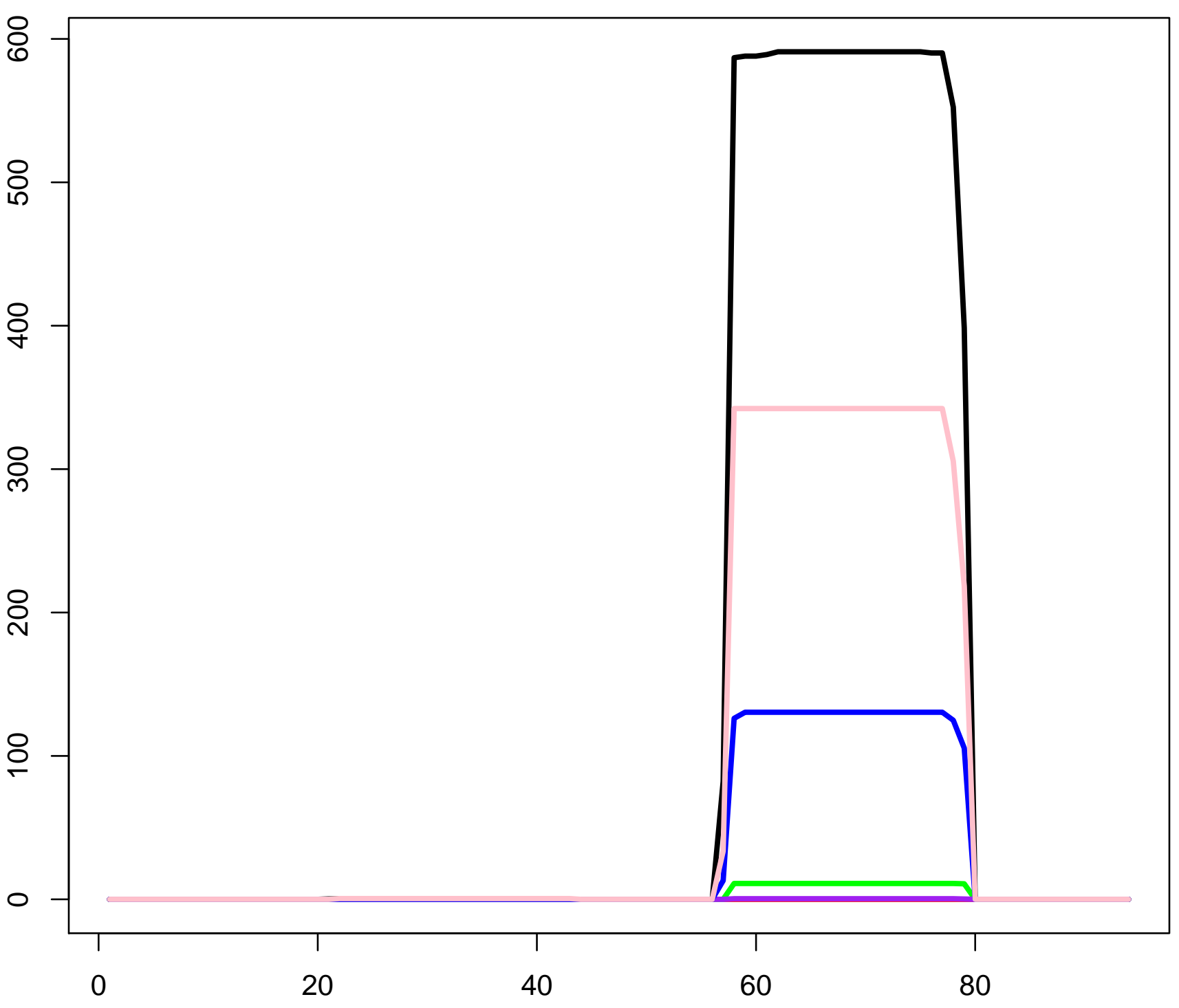


Scaffold70515_1376070-1376155(-) mir-205a

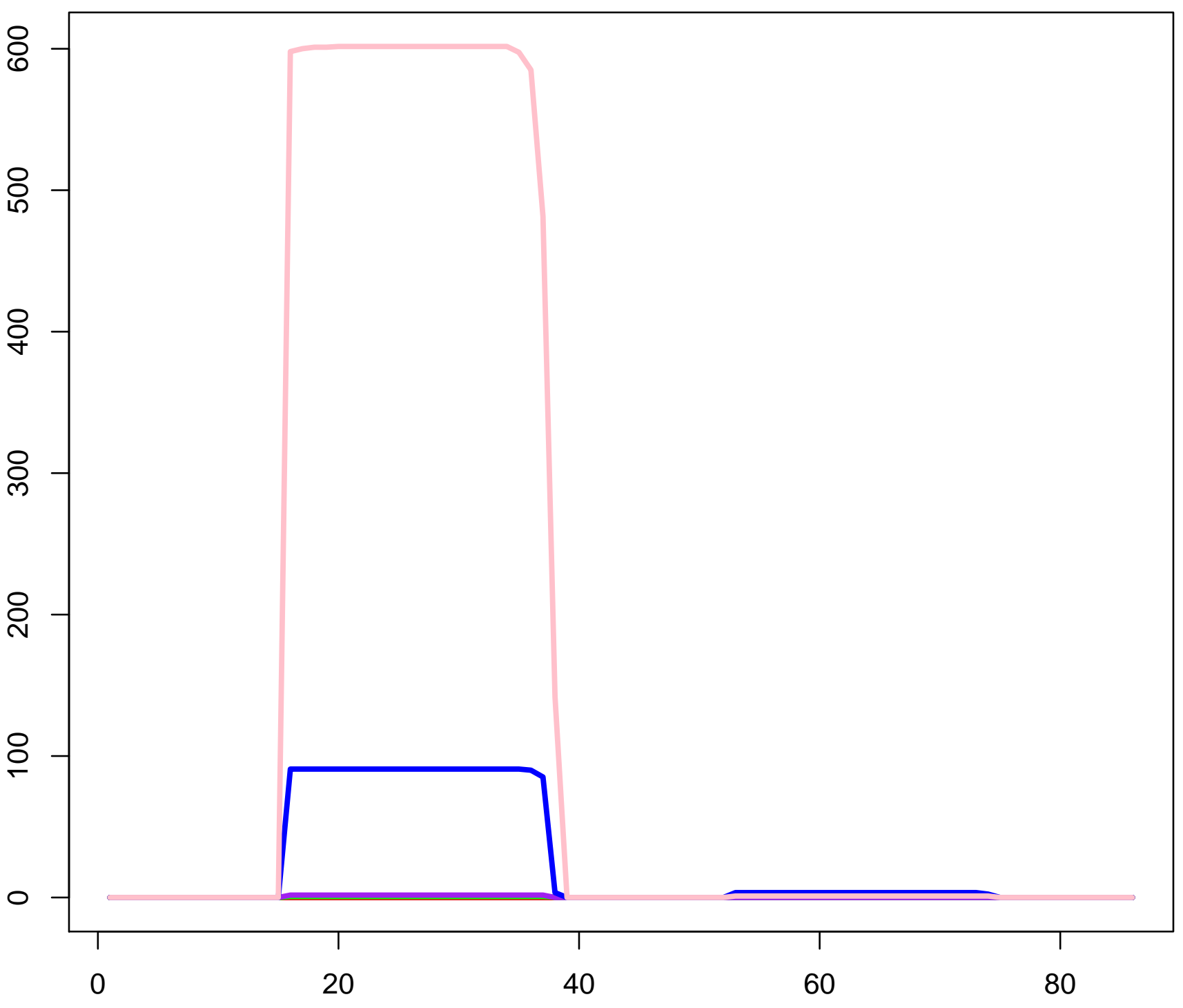


Scaffold7113_43709-43797(-) mir-124a-1

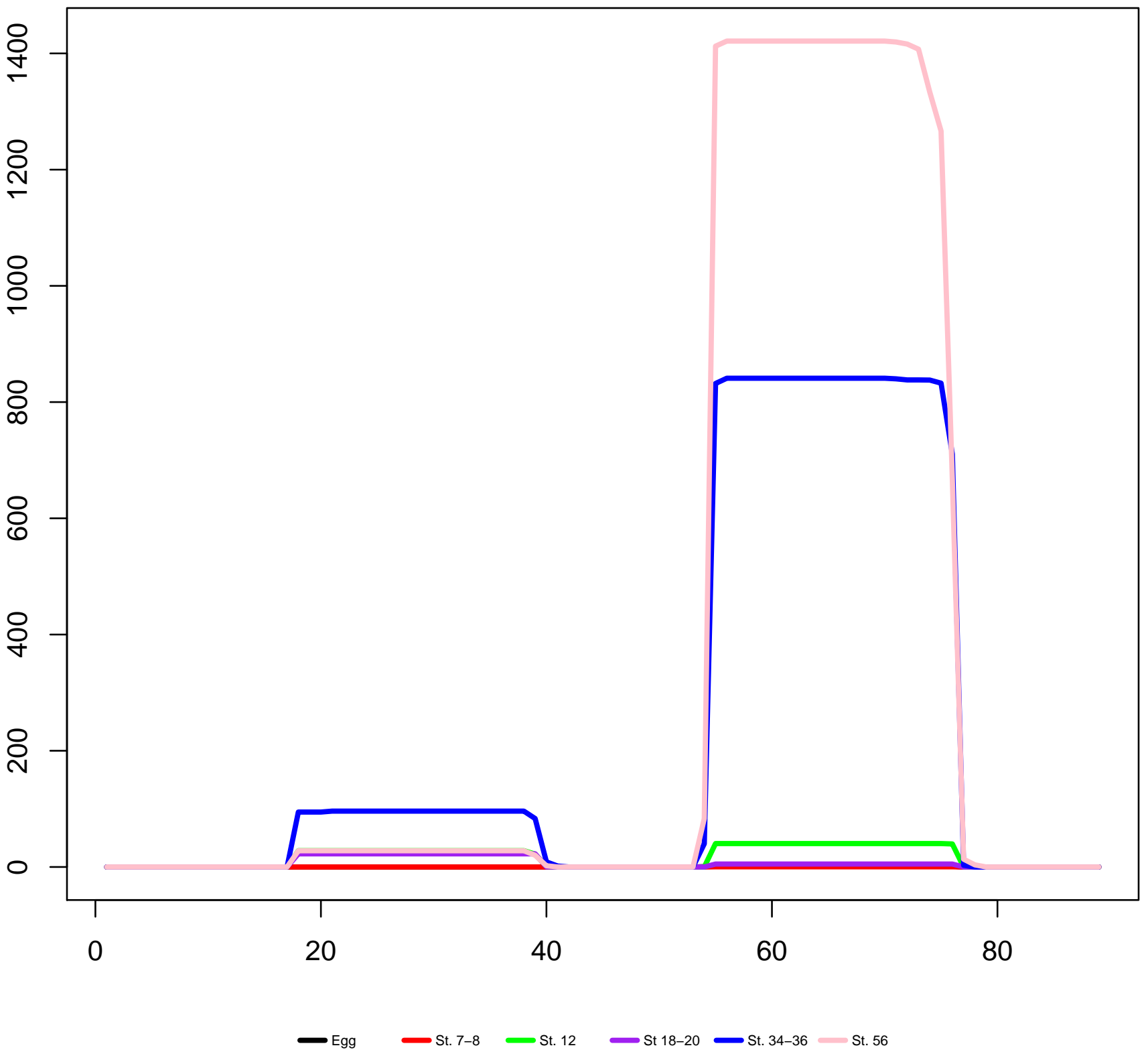




\section{Scaffold72111_301080-301156(+) mir-130c}

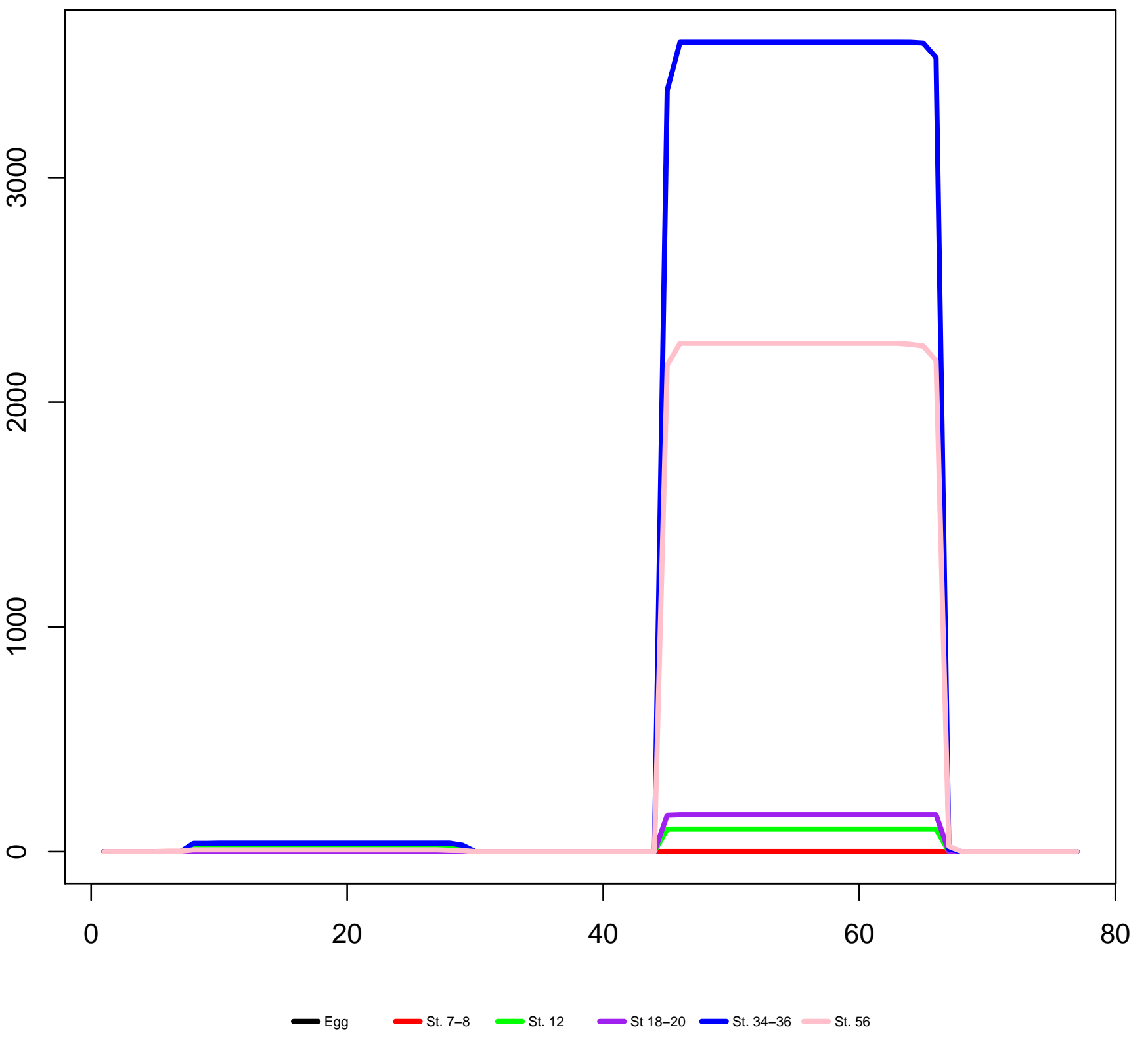


Scaffold72111_308258-308336(+) mir-454

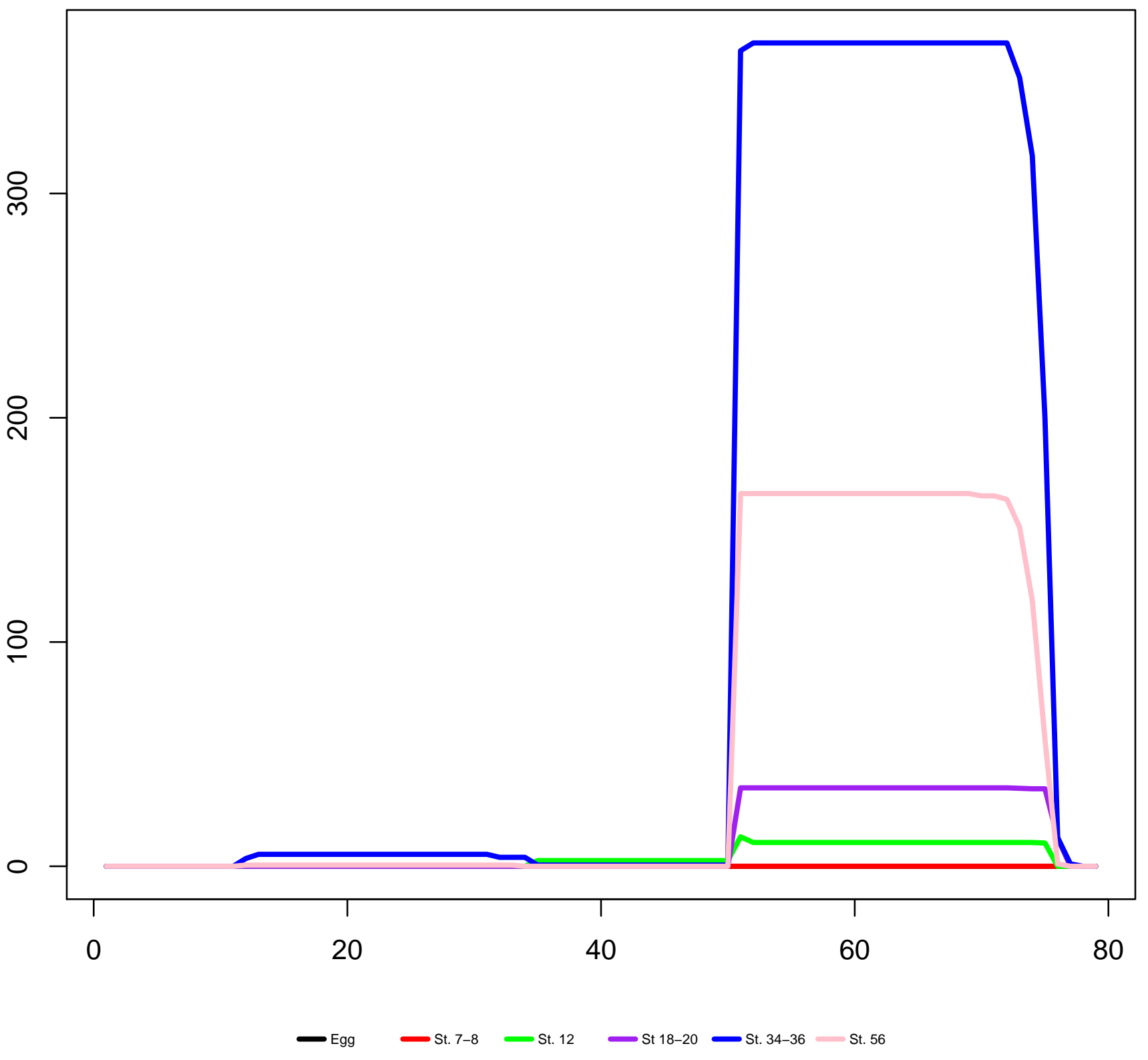


Scaffold72111_310269-310340(+) mir-130b

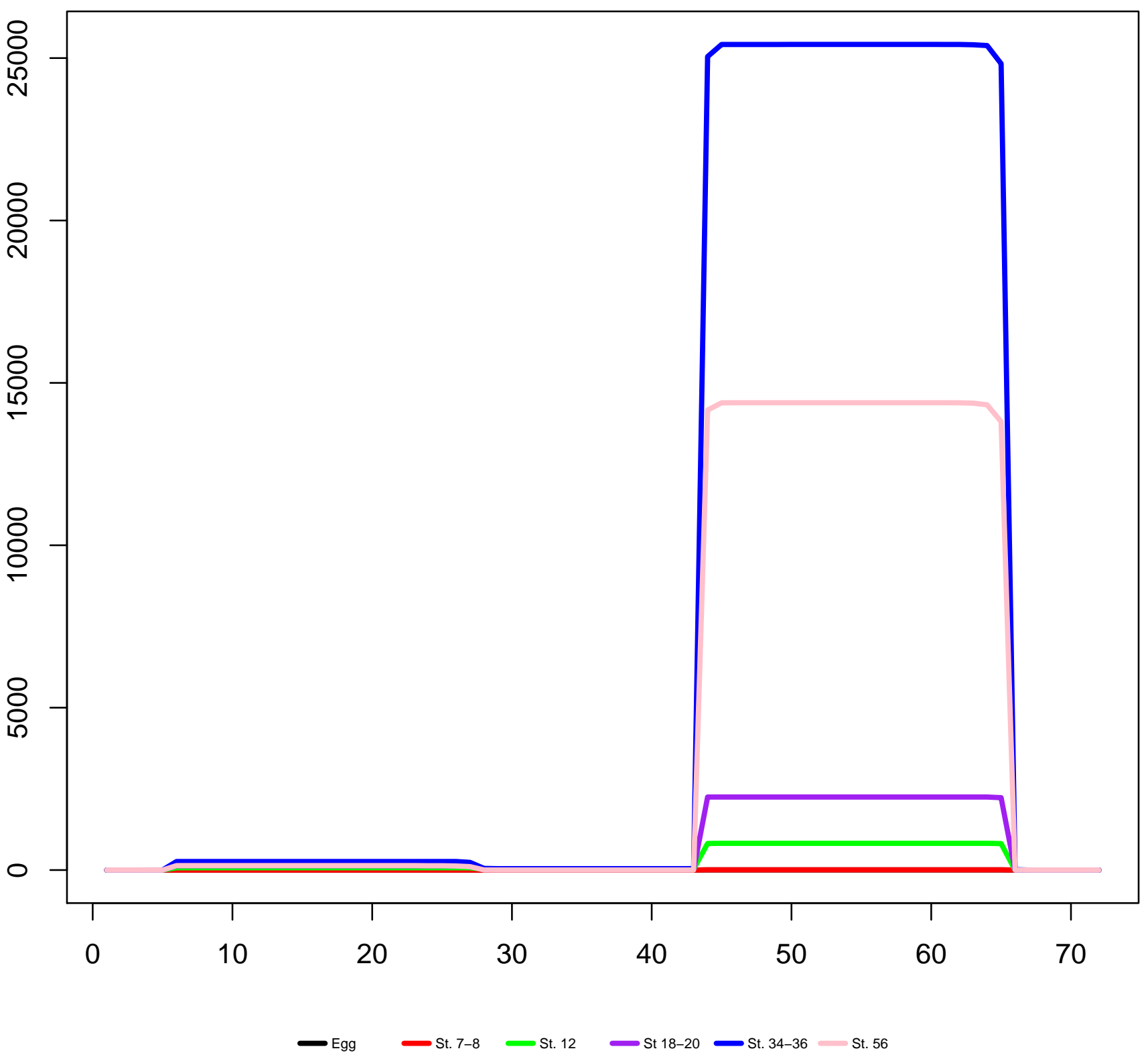


Scaffold7304_401607-401687(-) mir-208

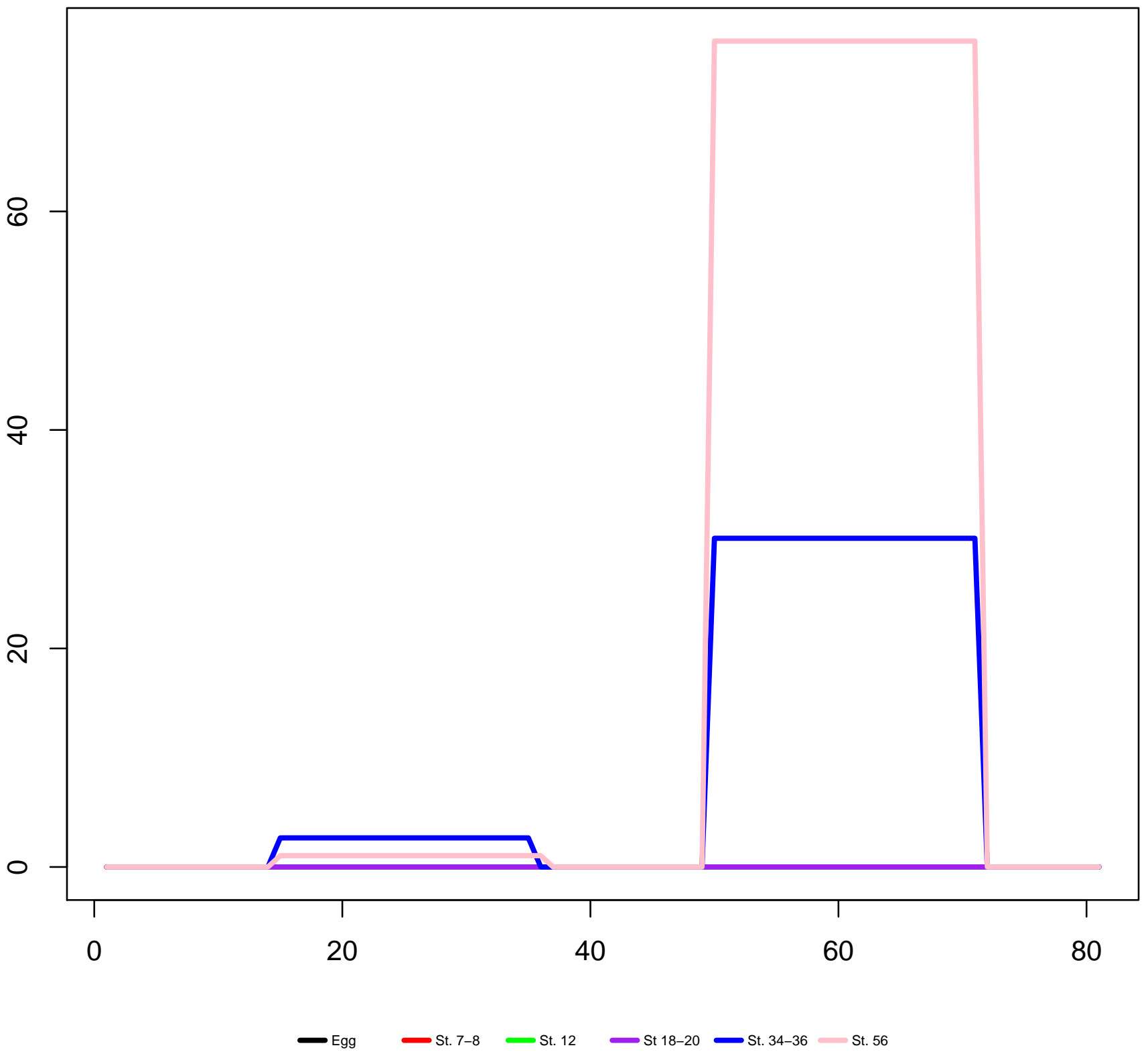


Scaffold7316_1322426-1322500(-) mir-2985a-1

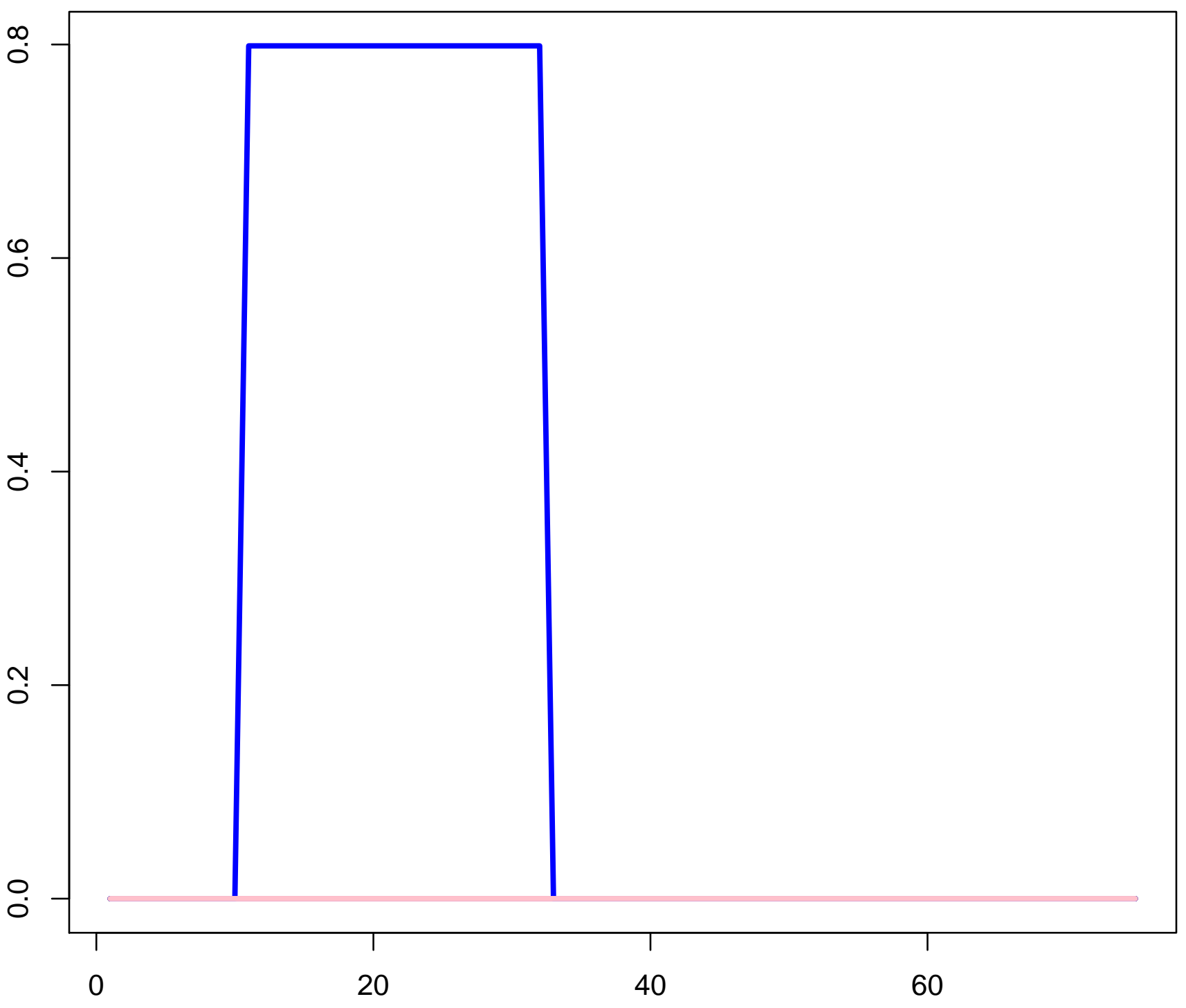




\section{Scaffold7562_102702-102780(-) mir-456}

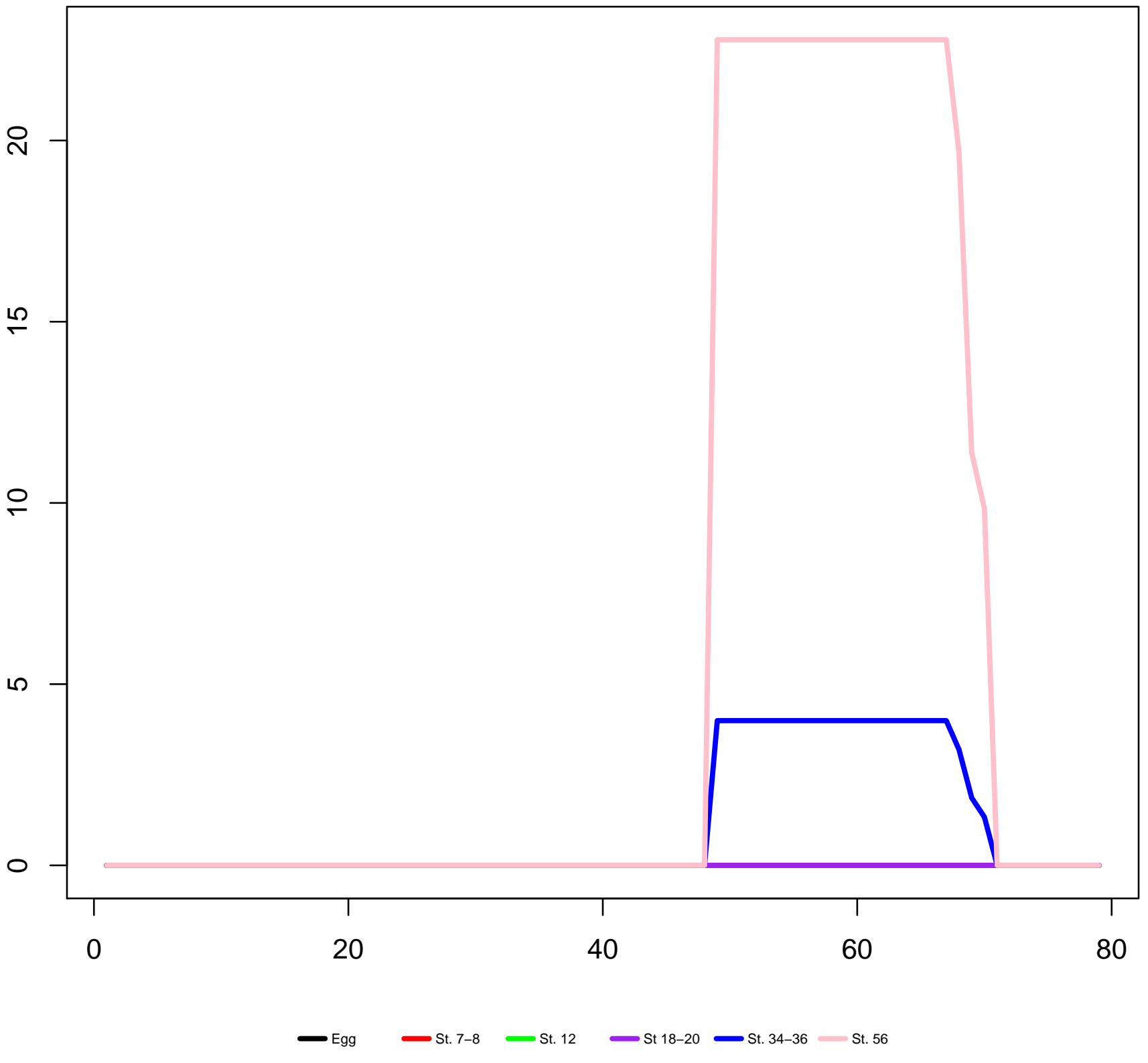


Scaffold7599_72518-72607(-) let-7a

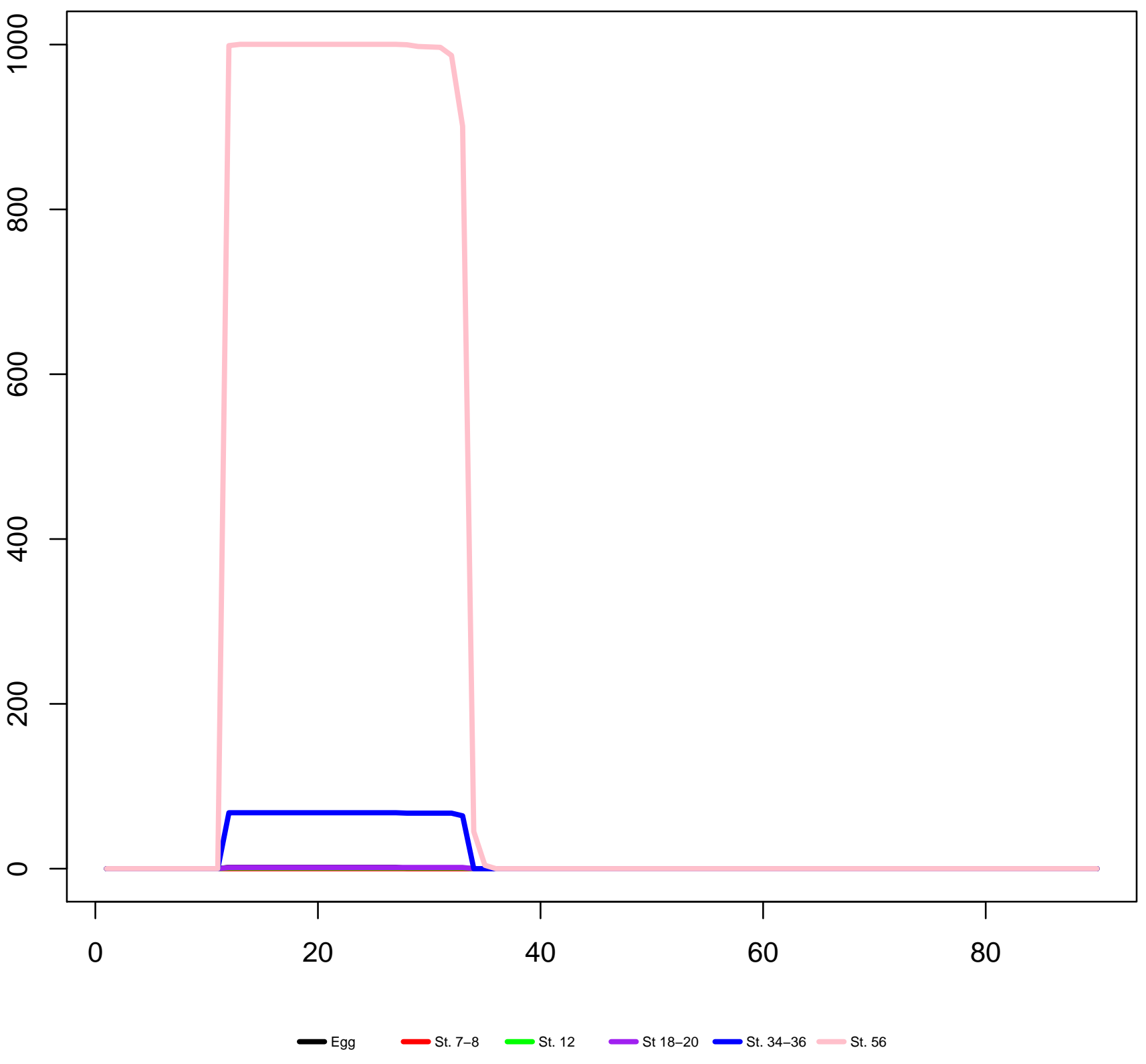


Scaffold7639_187716-187795(-) mir-26-2

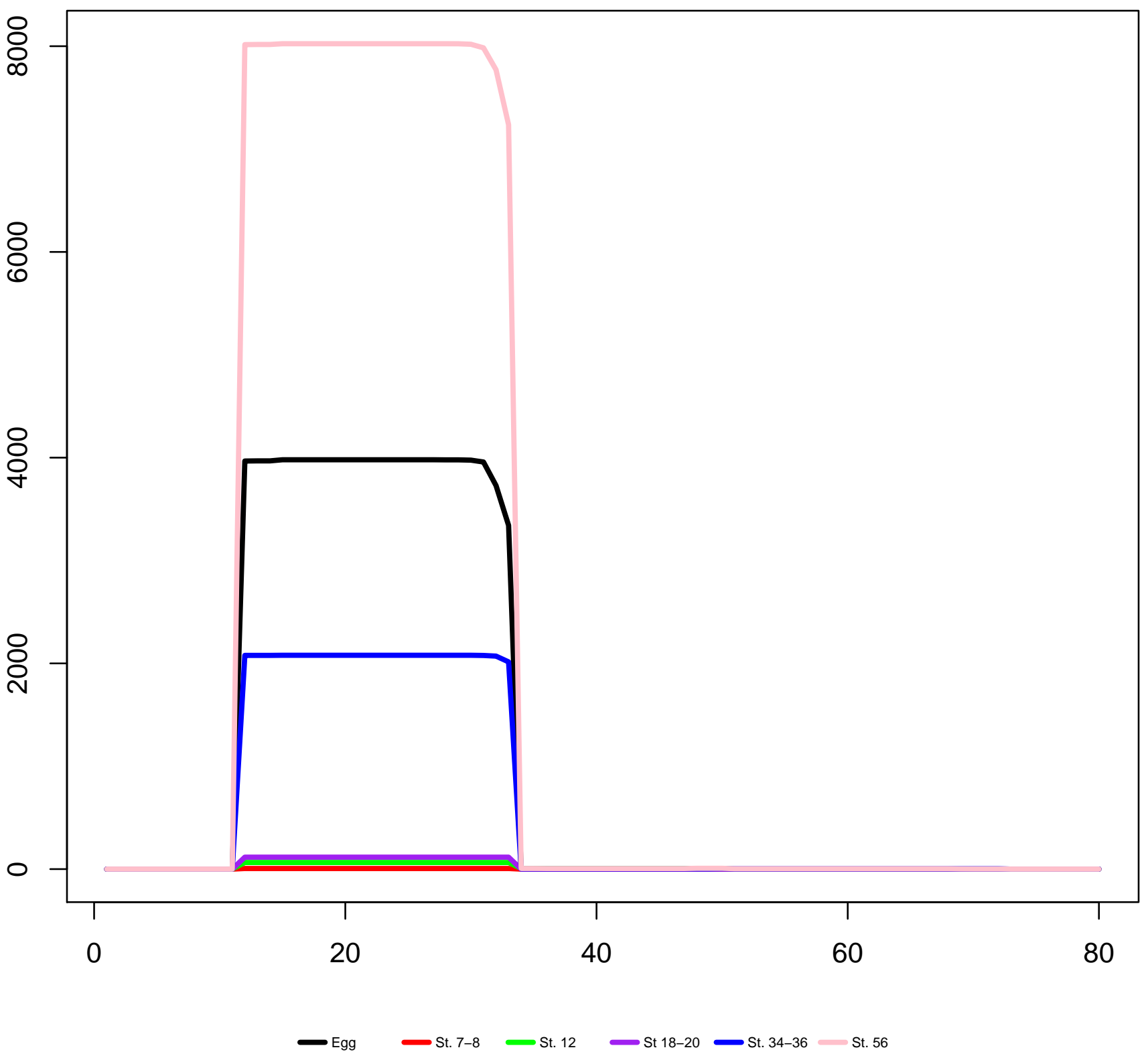




\section{Scaffold7676_140865-140946(-) mir-31a}

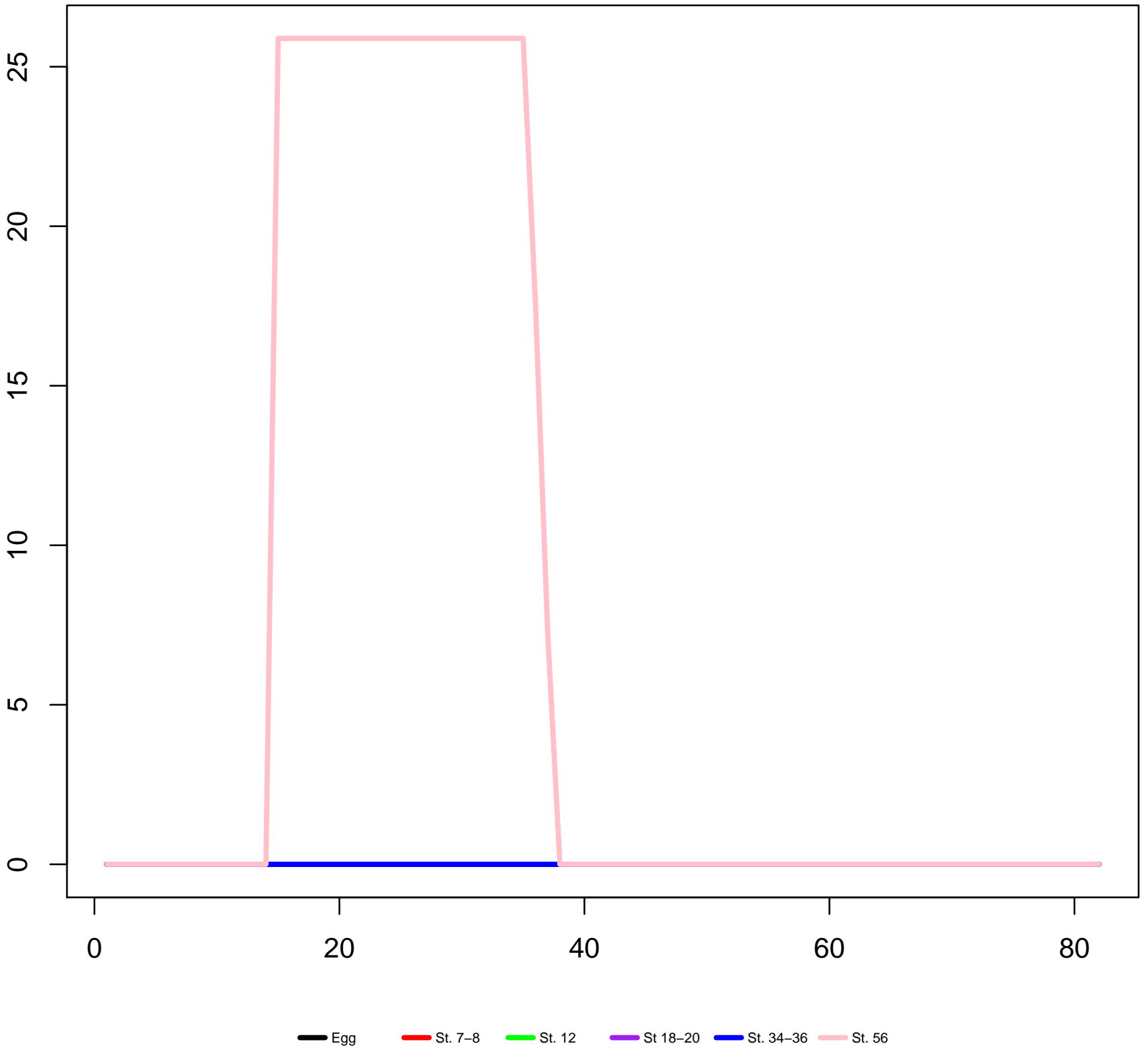


Scaffold78999_8199-8285(+) mir-192

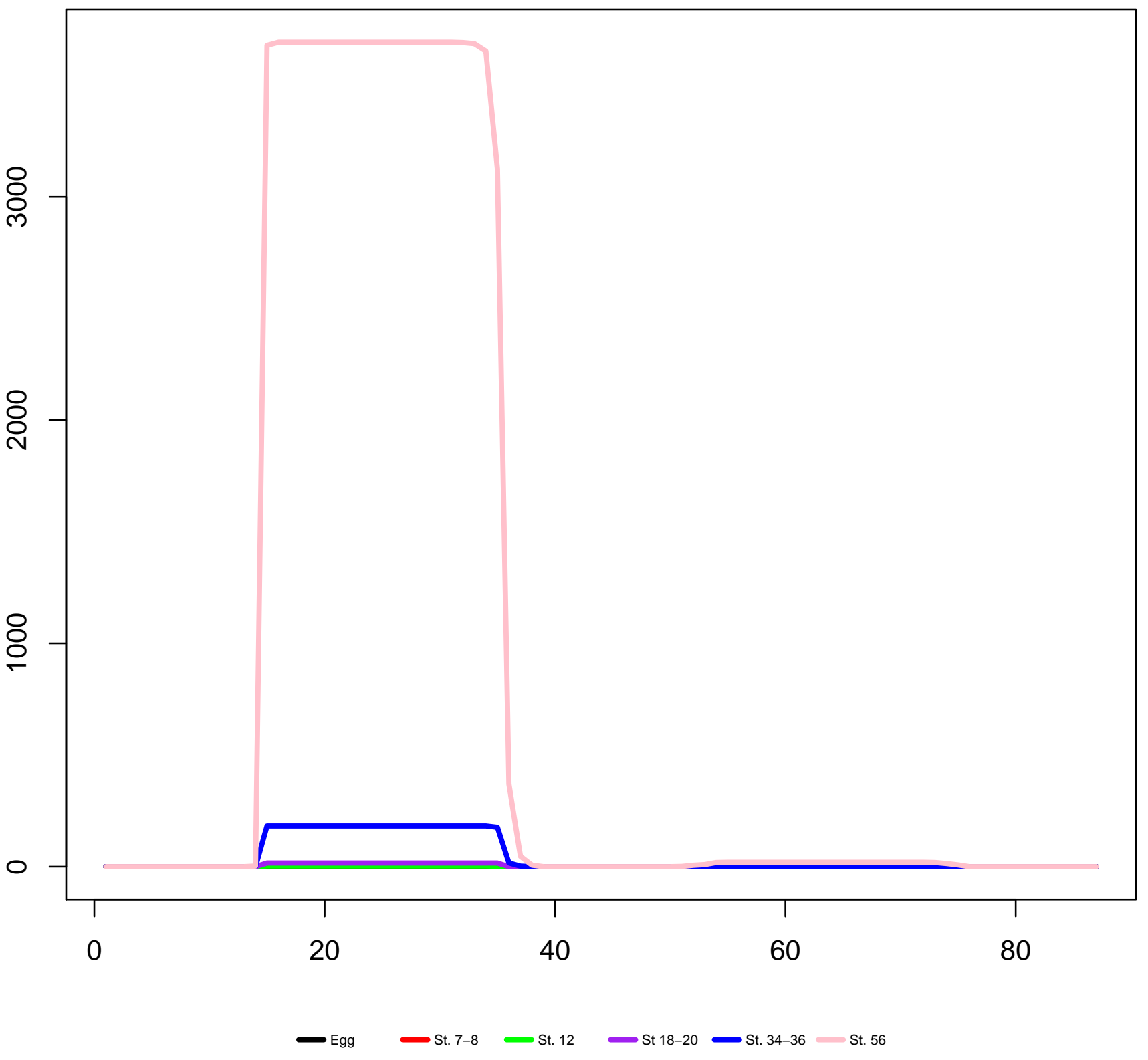


Scaffold80061_364823-364924(+) mir-30a

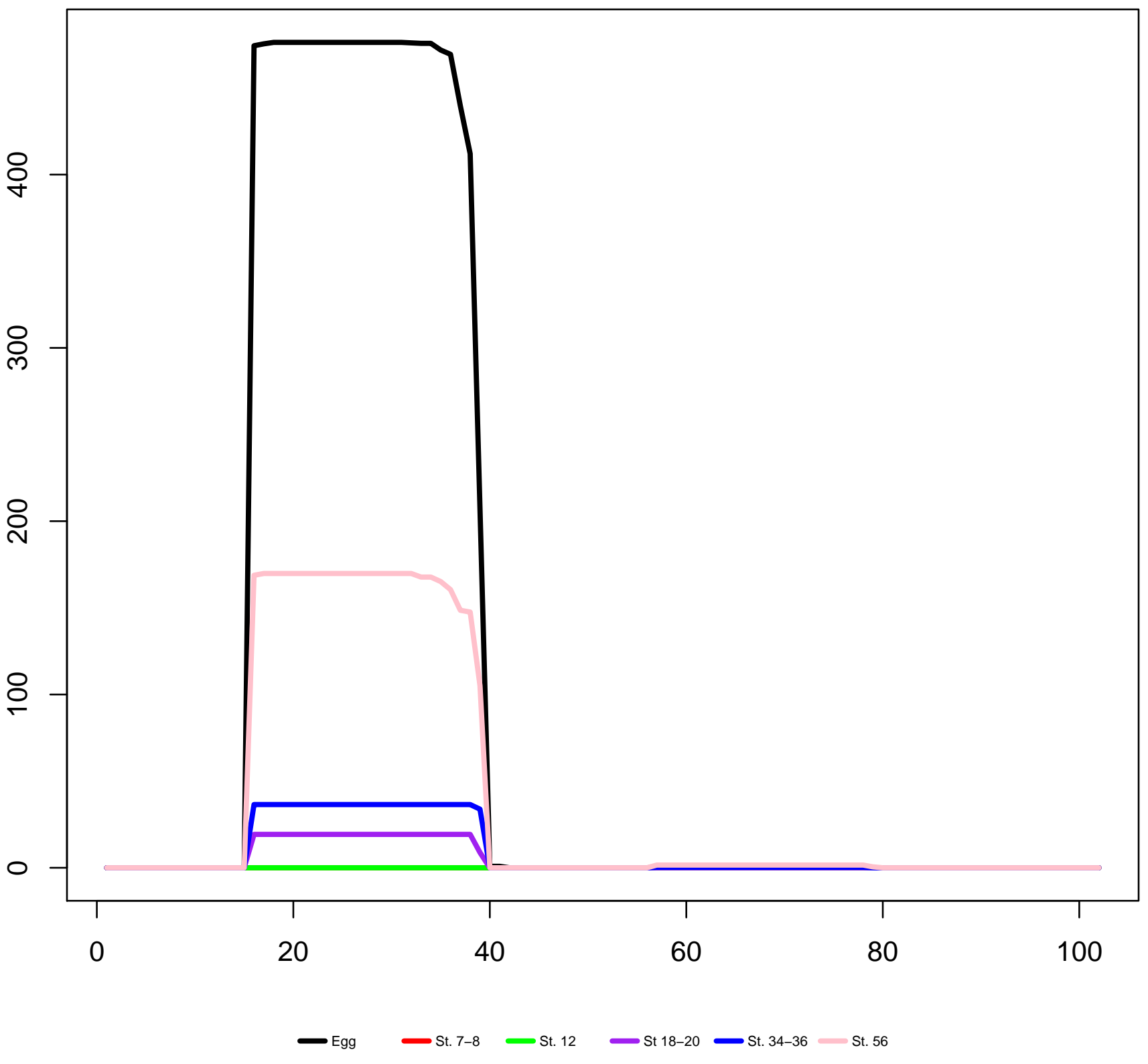


Scaffold8017_436113-436204(+) mir-428b

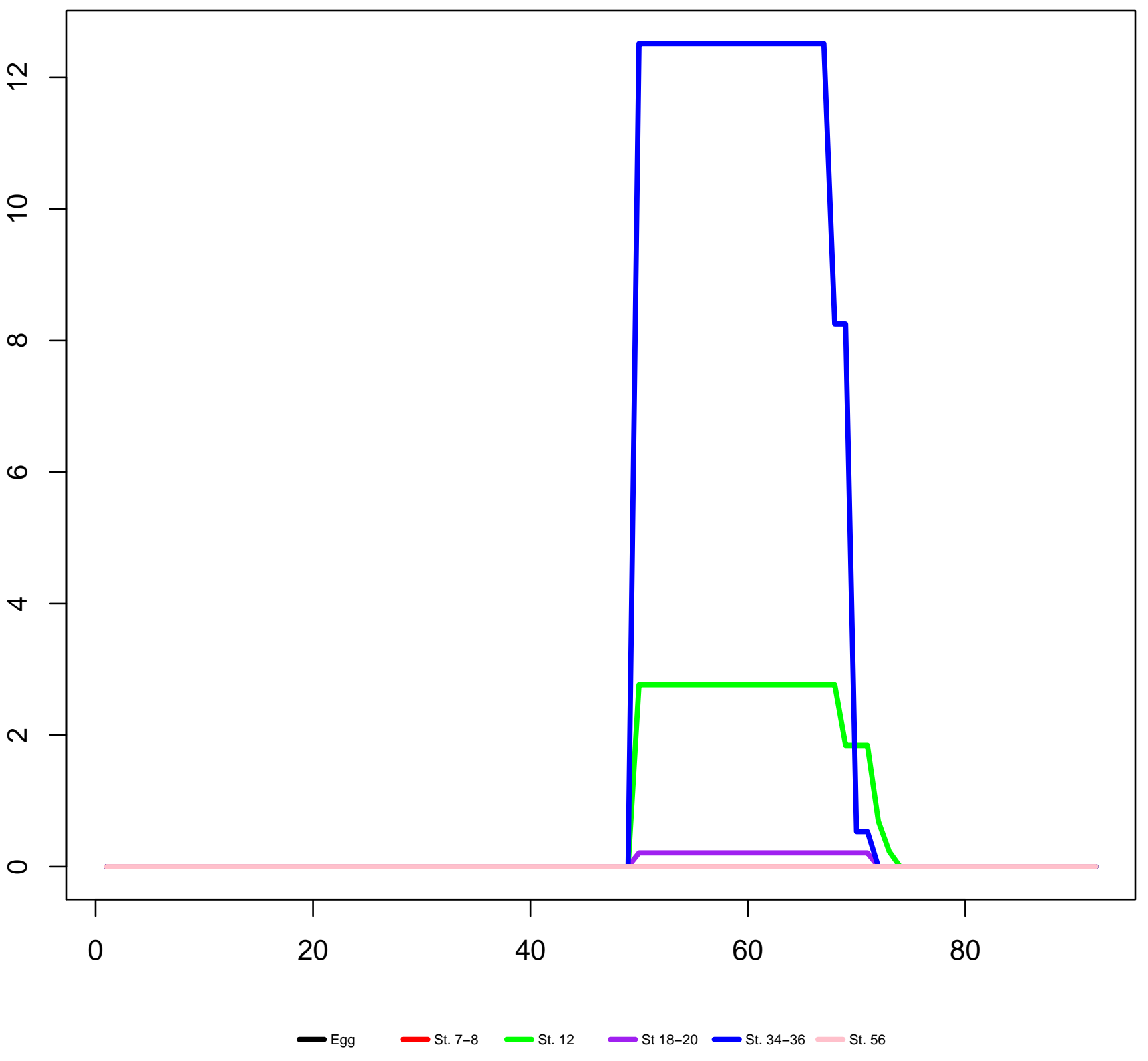


Scaffold80785_41262-41361(-) let-7a

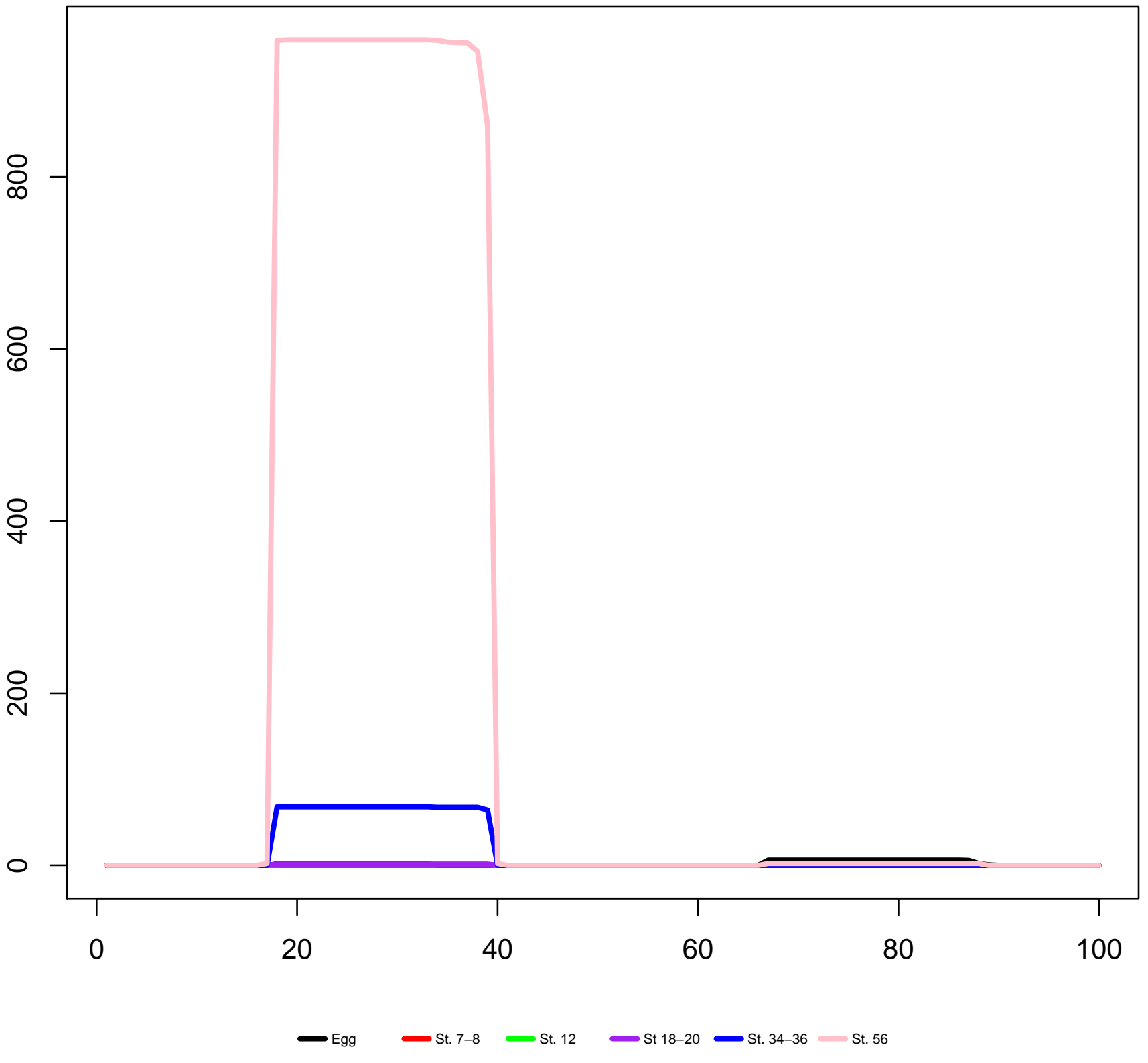


Scaffold81277_612609-612694(+) mir-148a

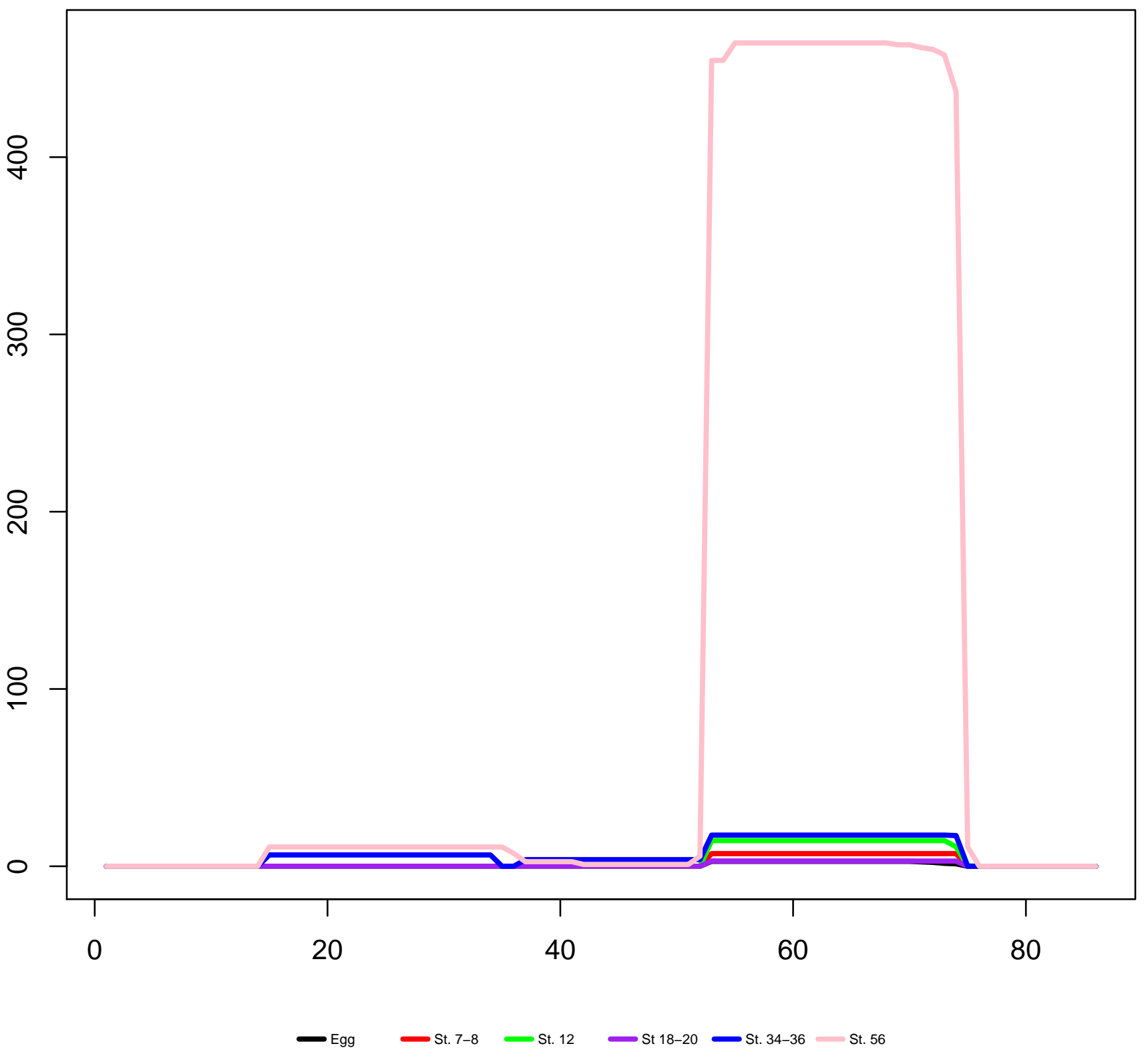


Scaffold8178_272800-272876(+) mir-2184

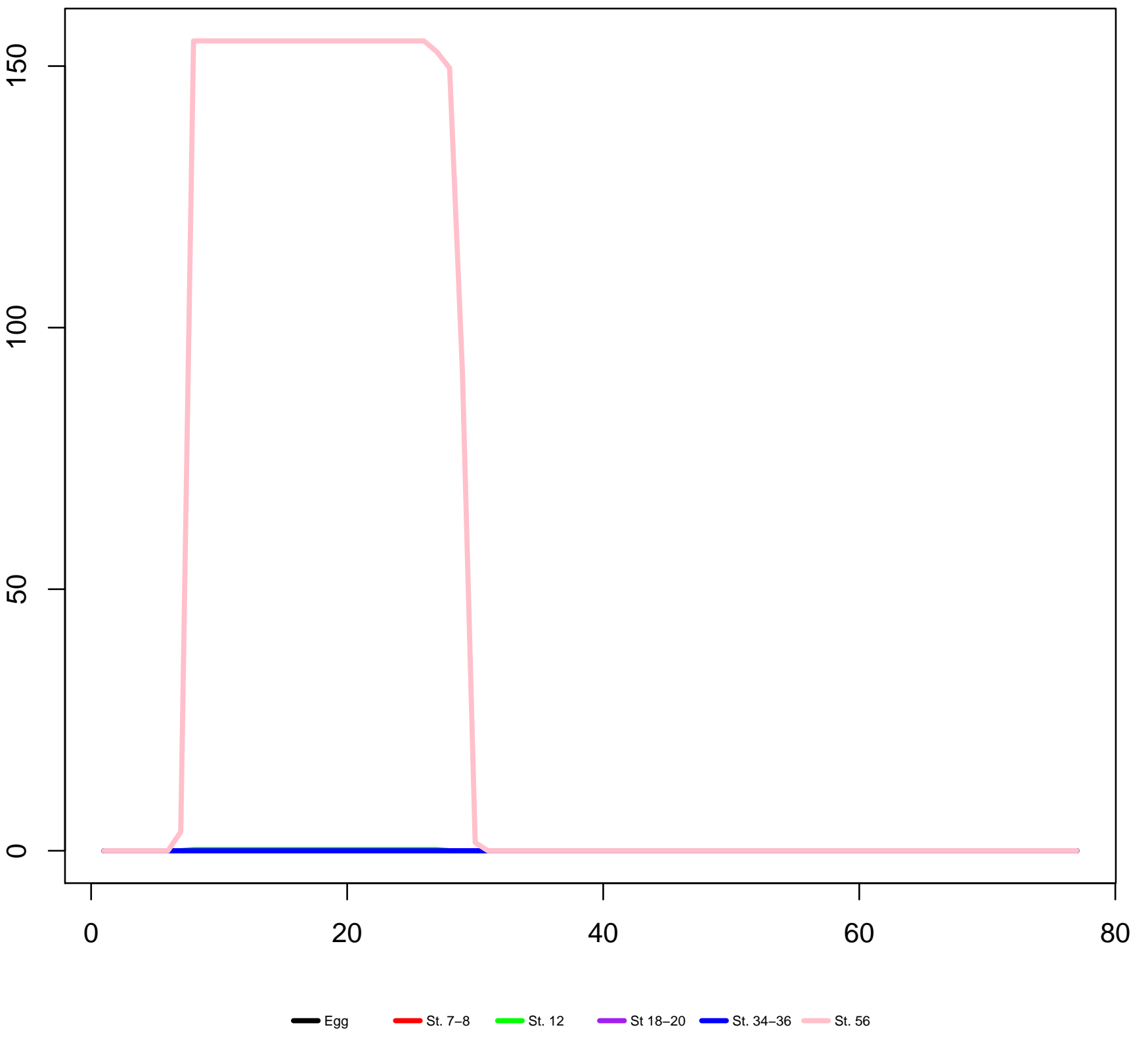




\section{Scaffold8178_306566-306653(-) mir-132}

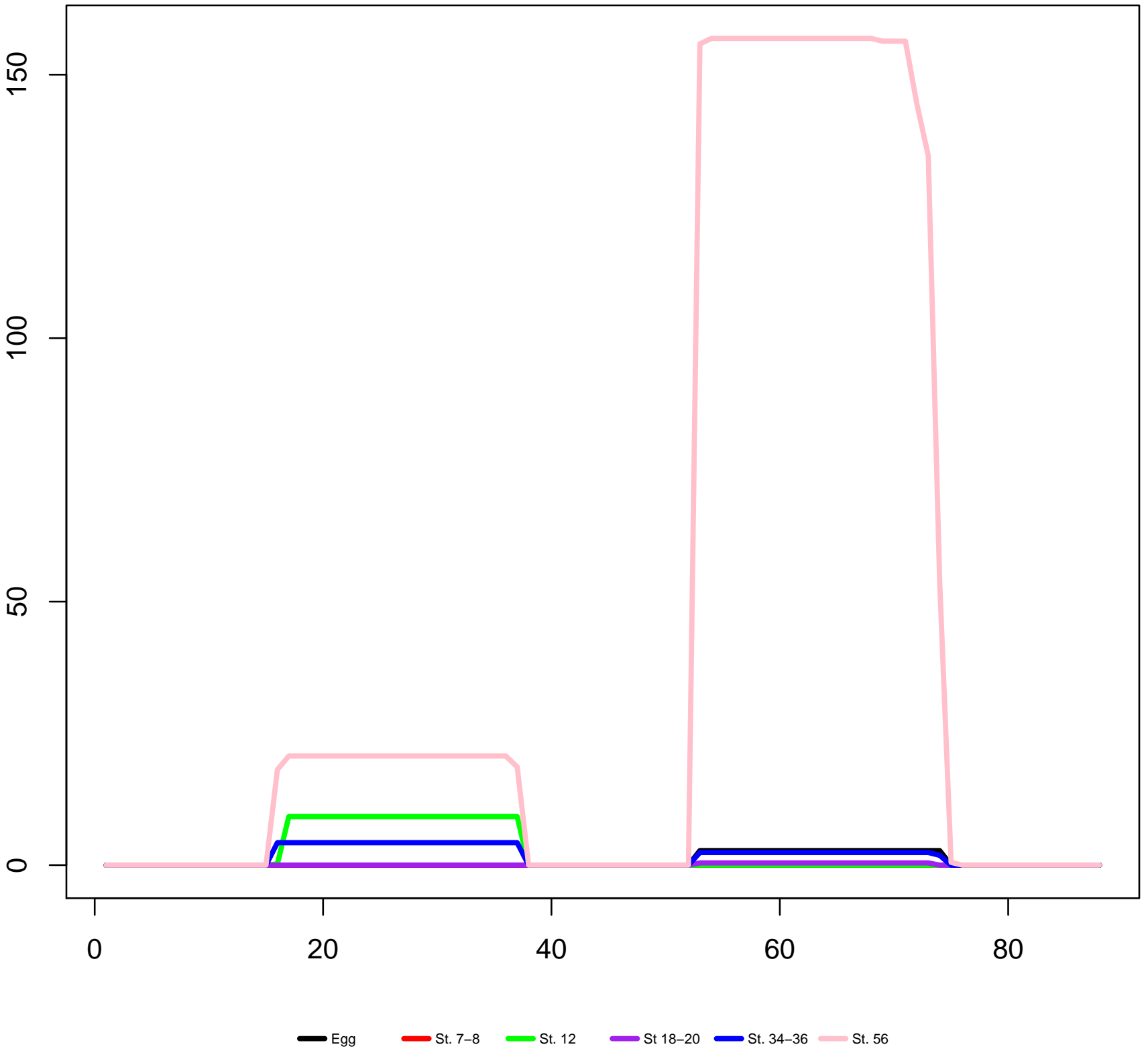


Scaffold8178_932823-932884(-) mir-144

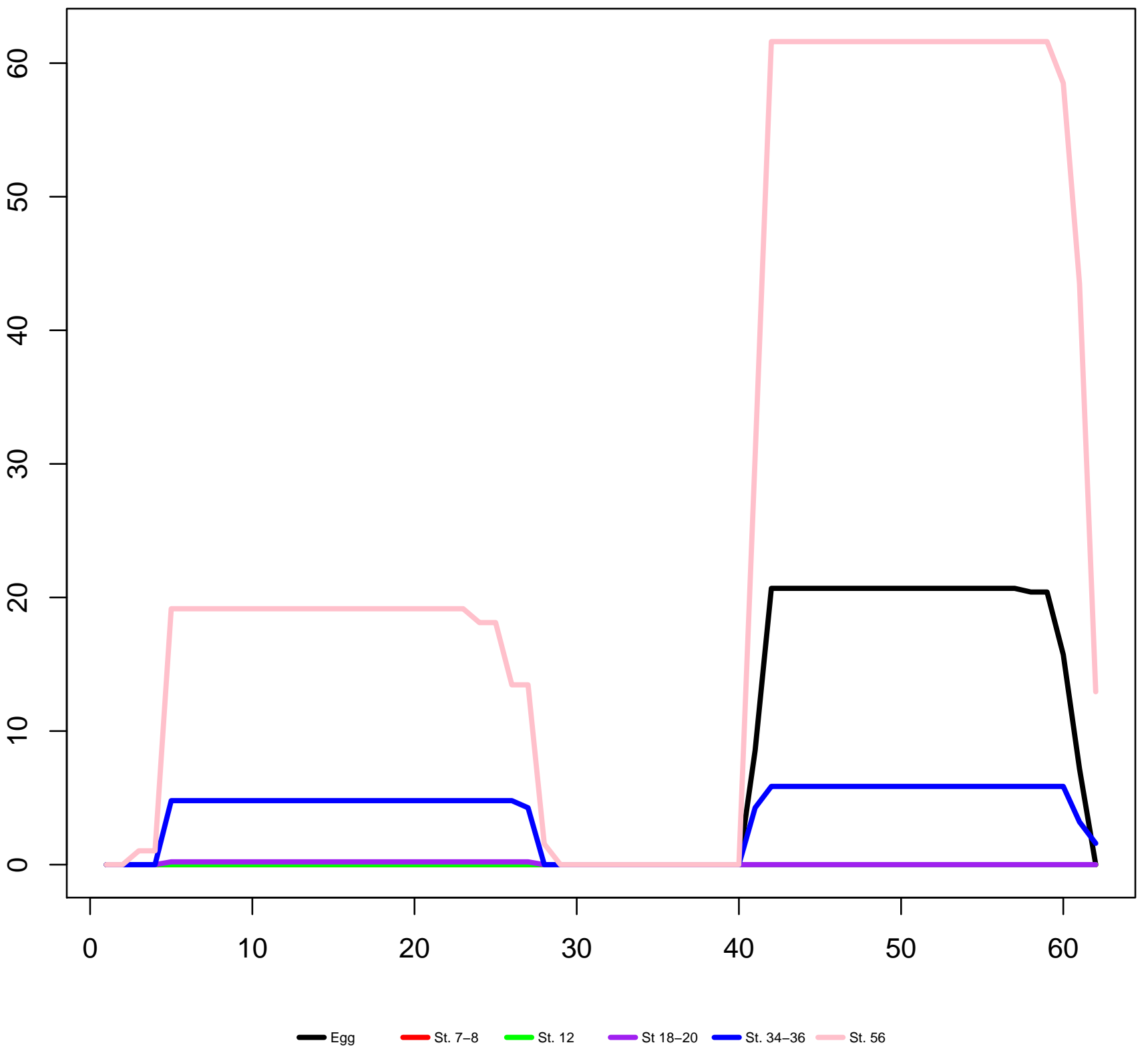


Scaffold82464_535672-535756(+) mir-191

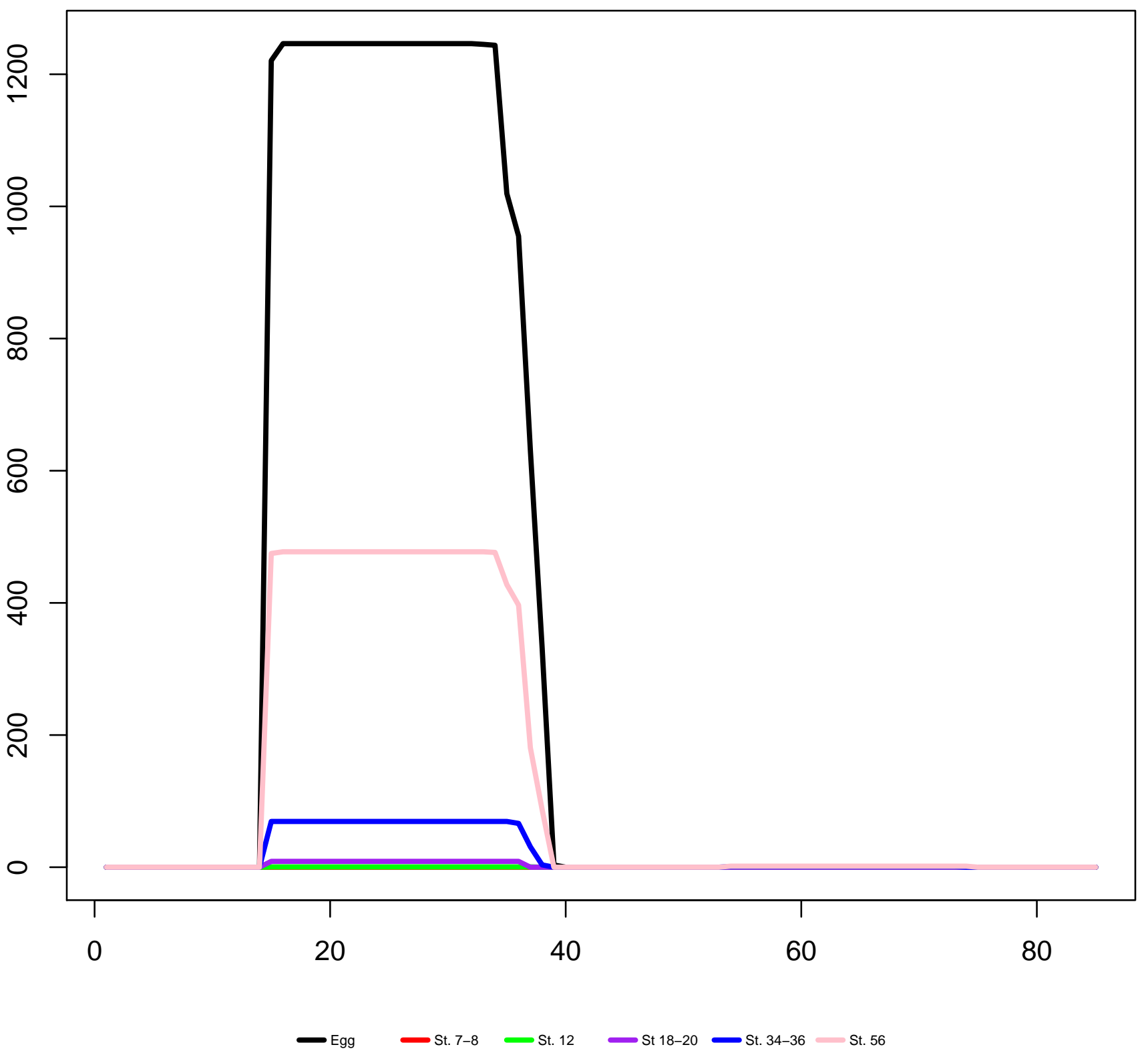


Scaffold83271_85812-85875(+) mir-150

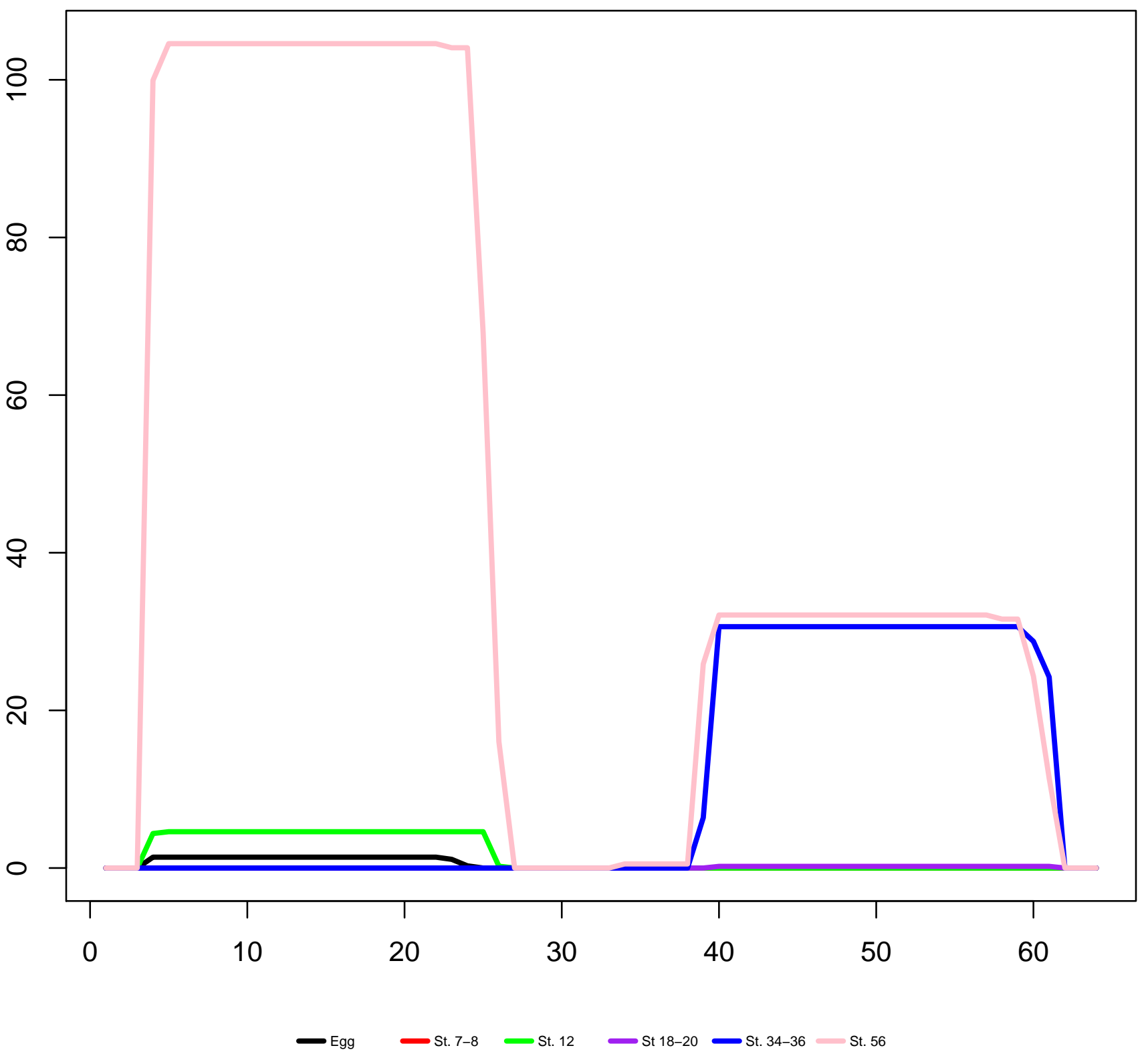


Scaffold84419_58429-58521(-) mir-146b

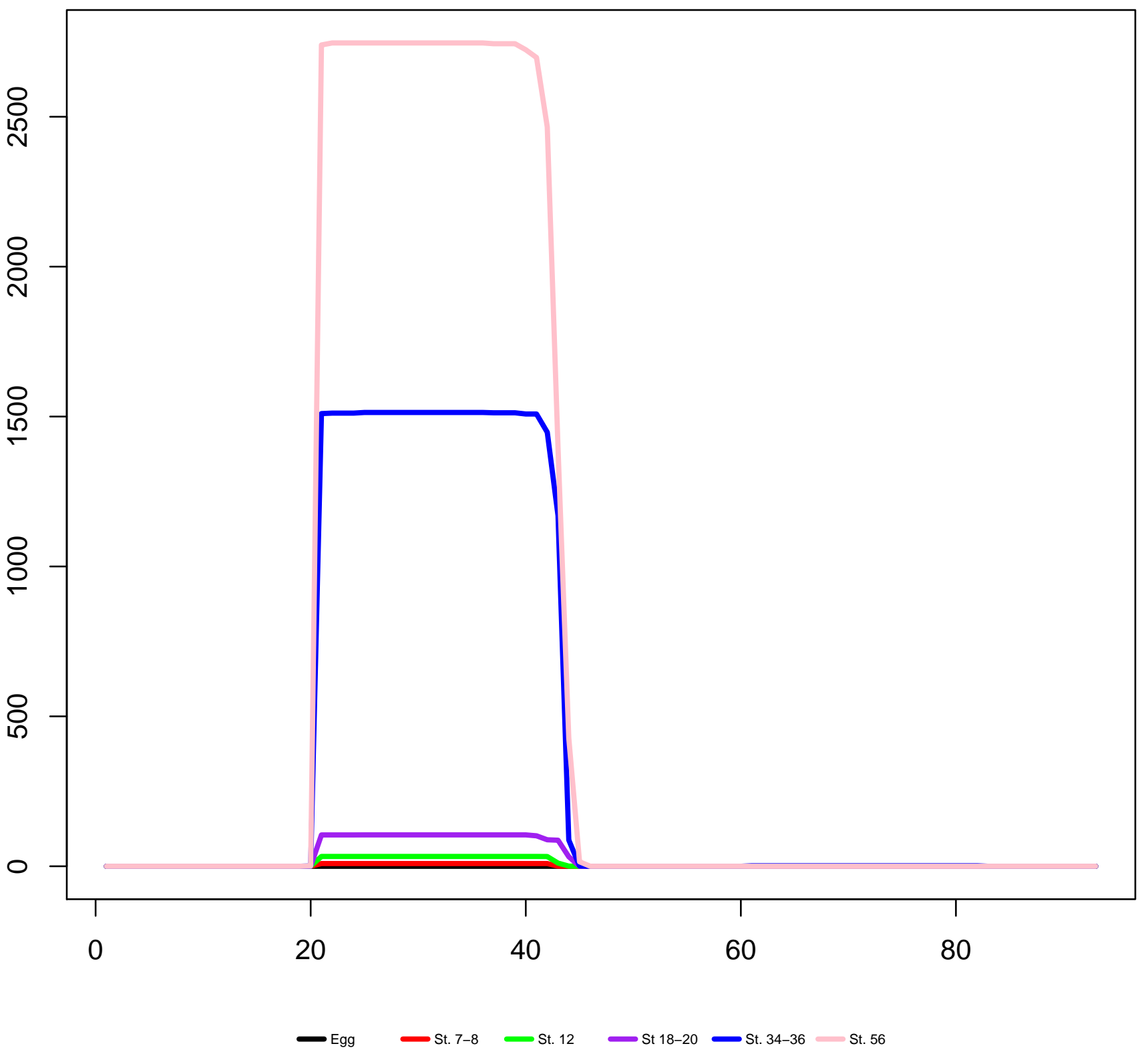


Scaffold854_740132-740216(-) mir-1306

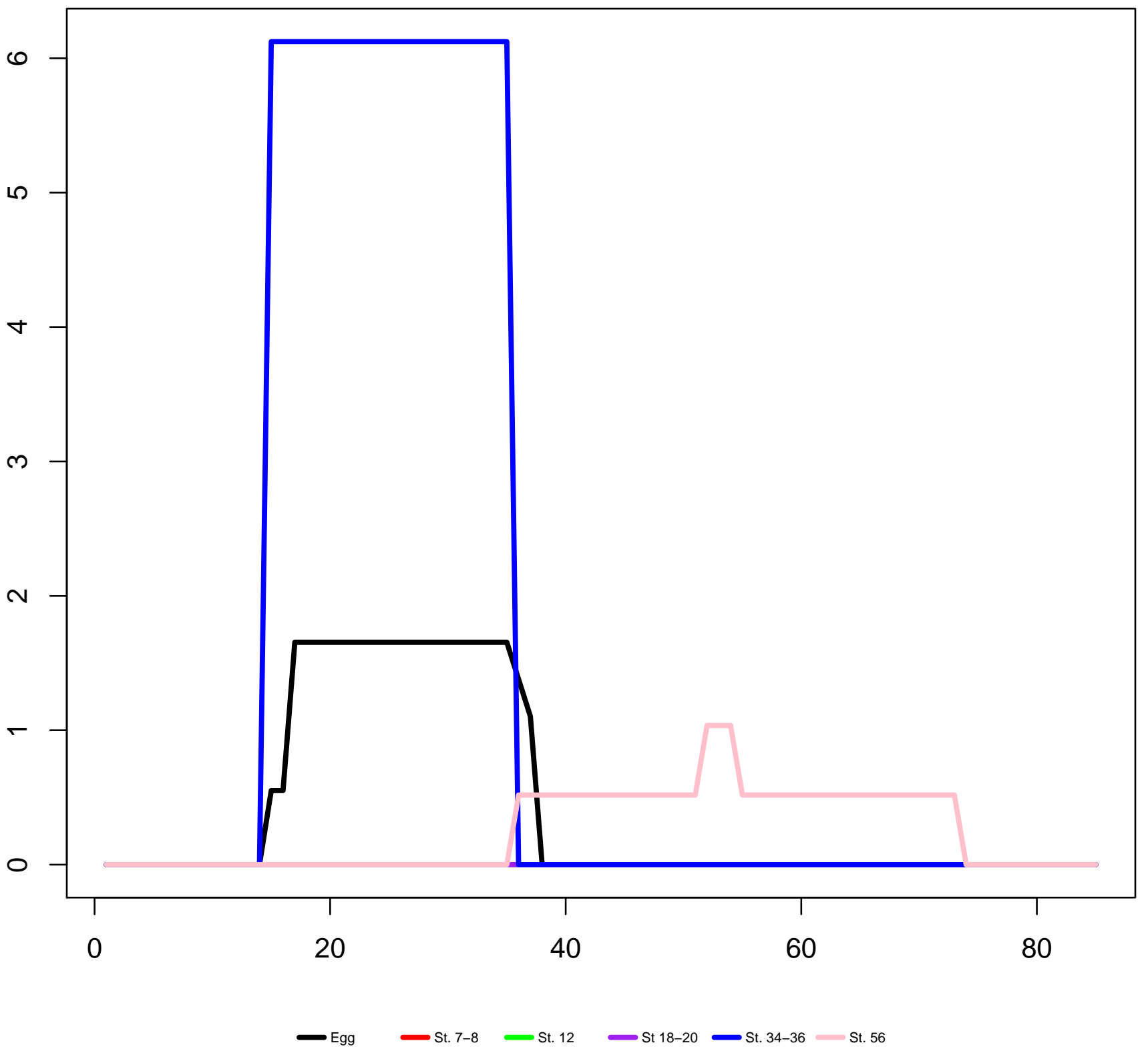


Scaffold854_740429-740519(-) mir-3618

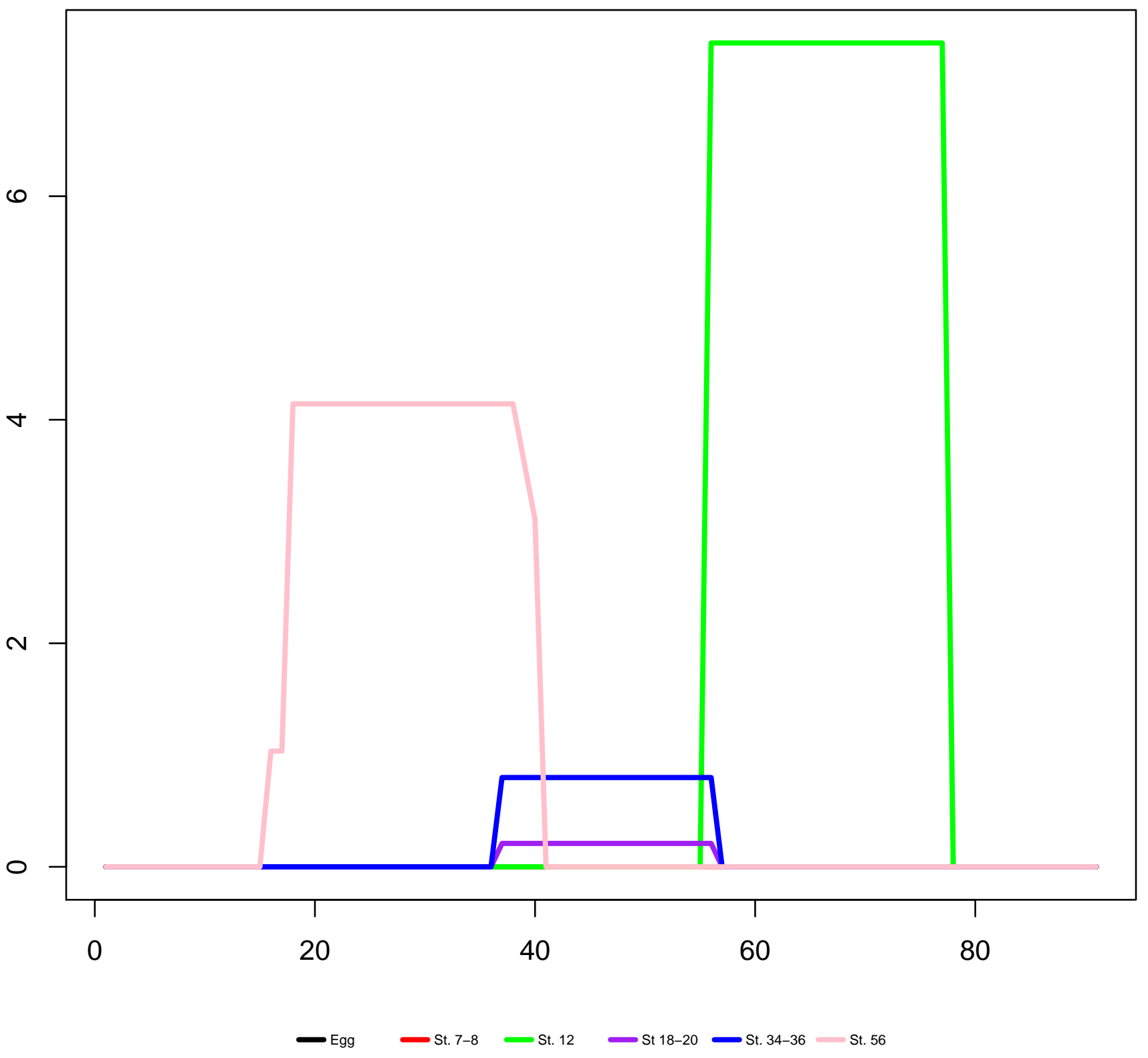


Scaffold8540_69100-69175(+) mir-100

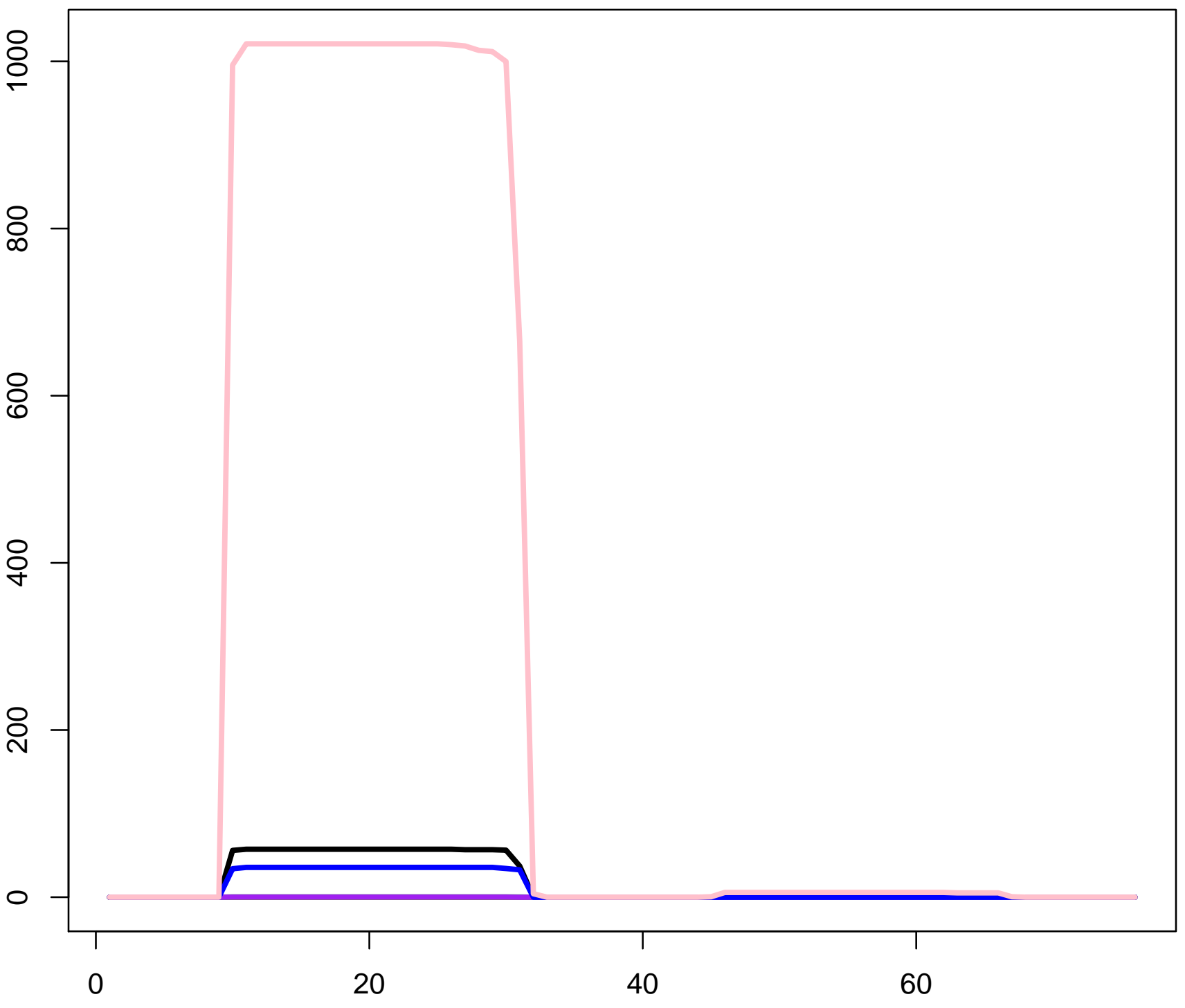


Scaffold8540_70931-71015(+) let-7c-2

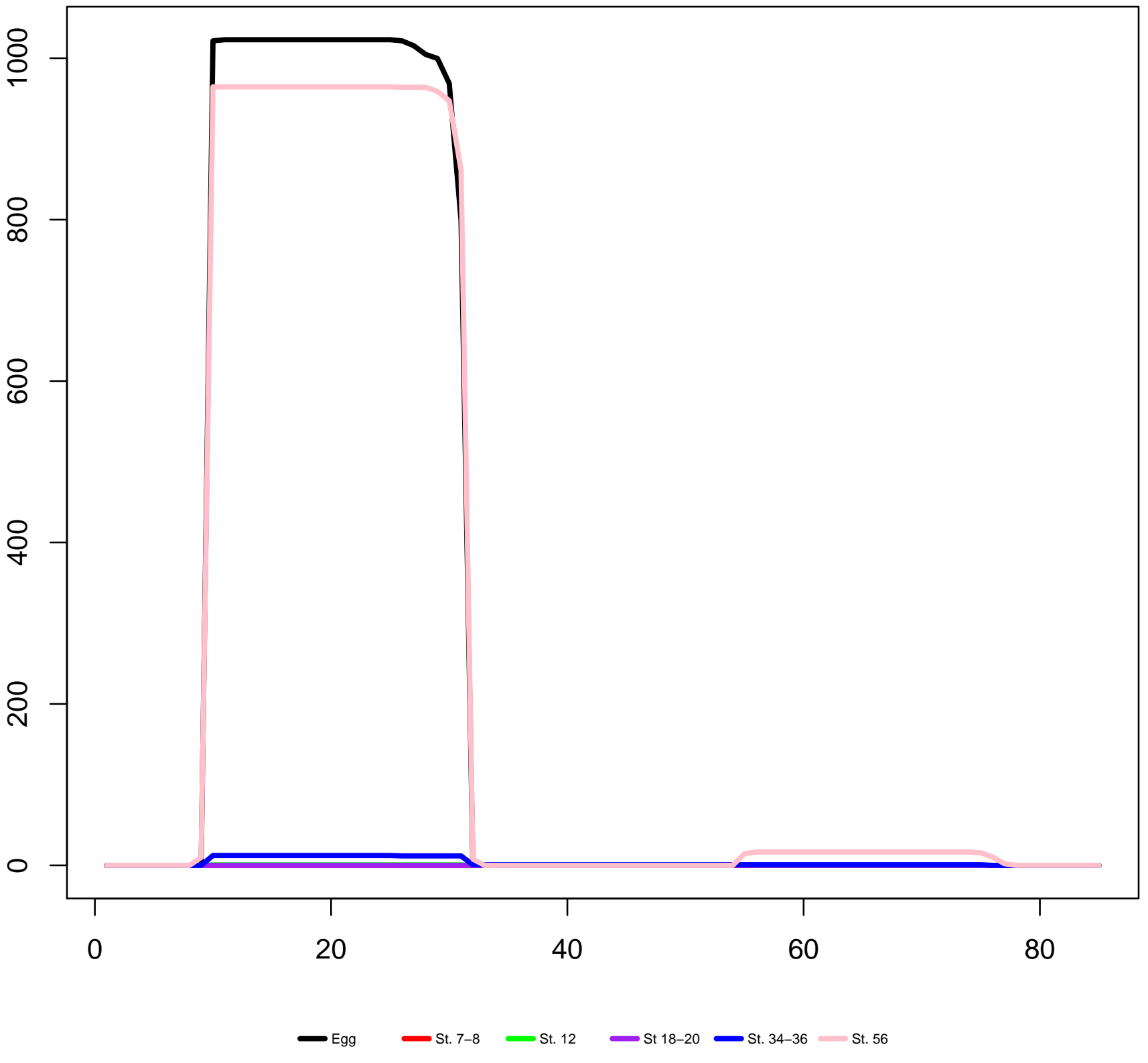


Scaffold8555_784666-784763(+) mir-9-1

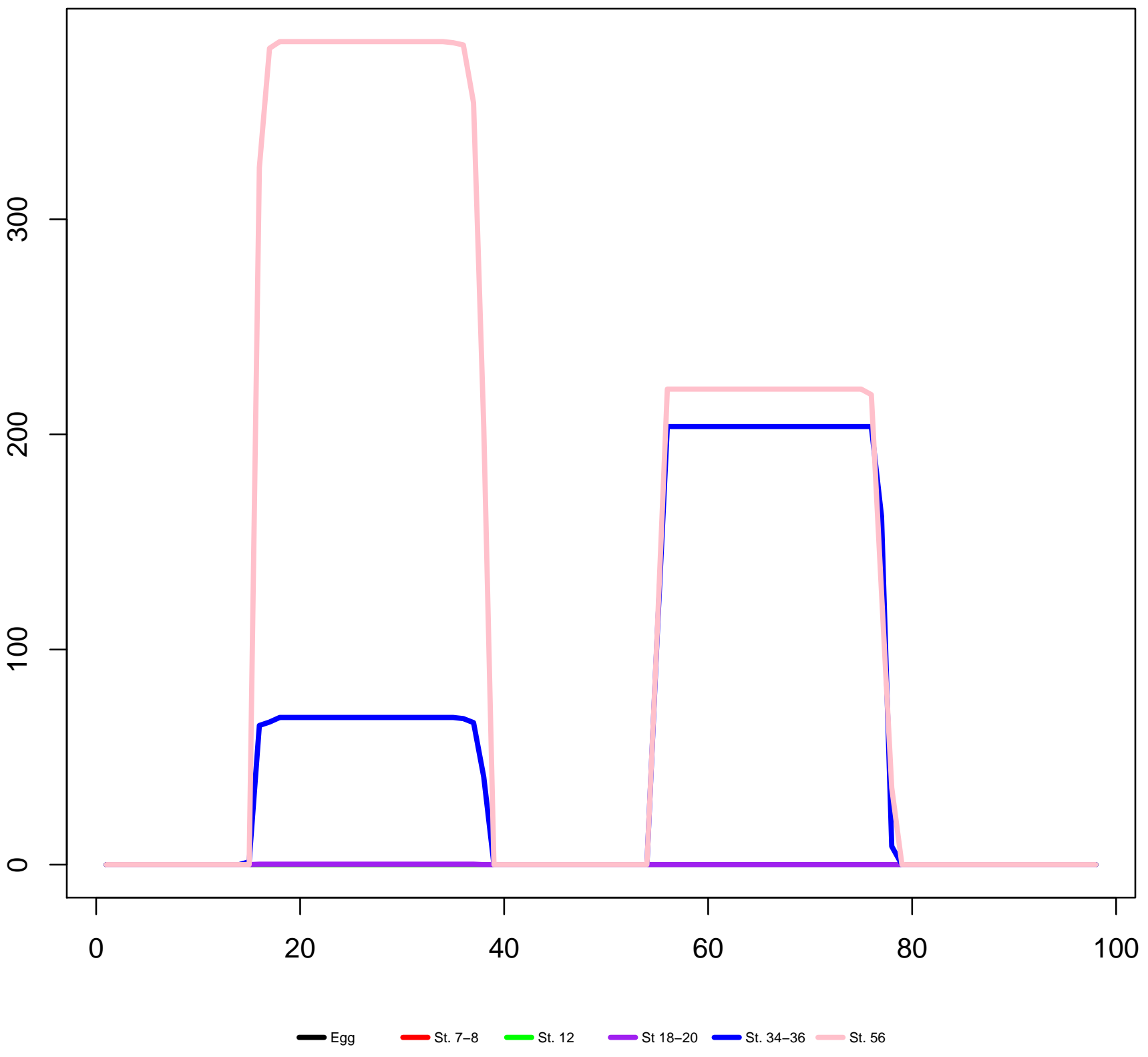


Scaffold85909_75338-75438(+) mir-137a

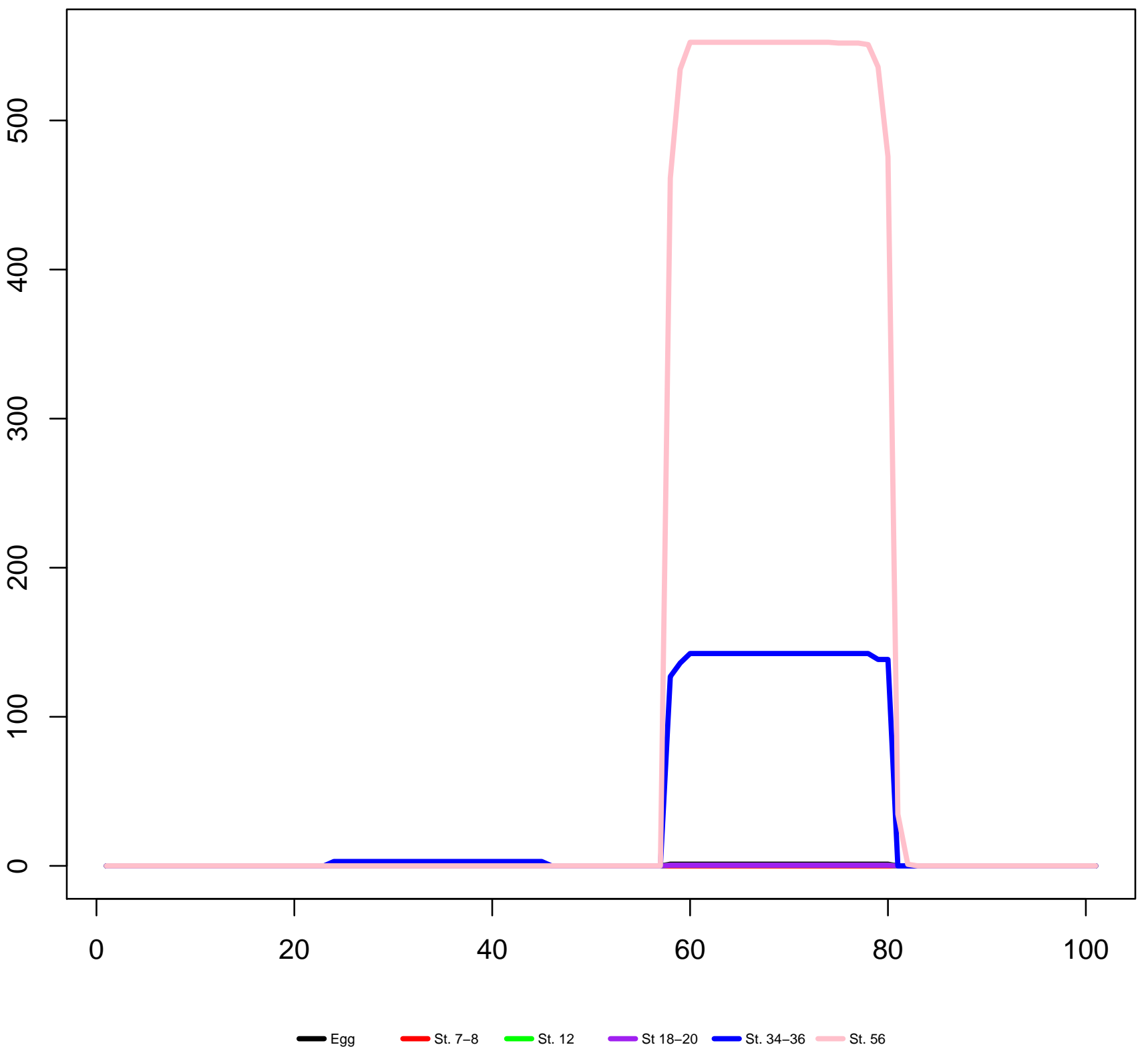




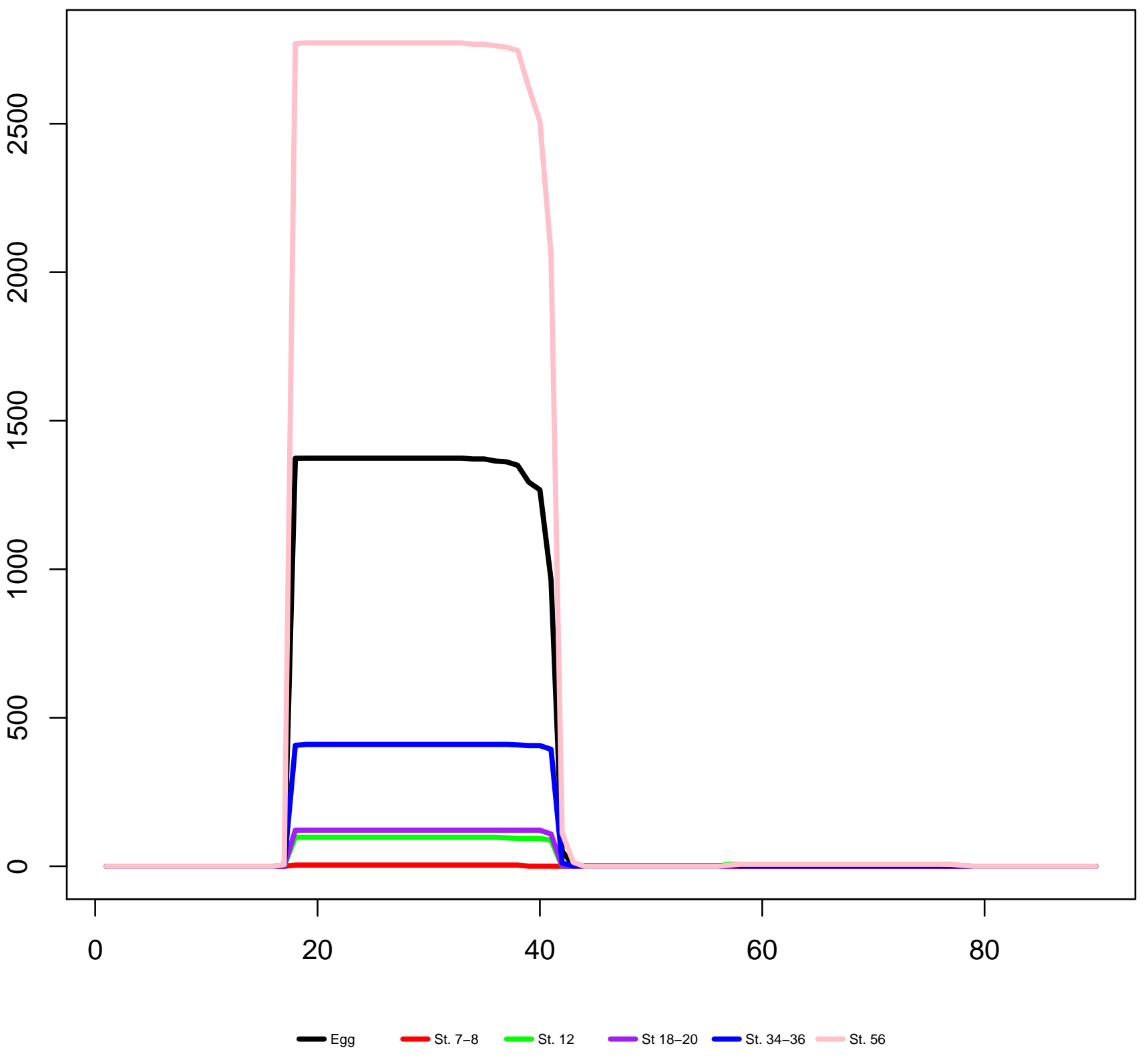


Scaffold91500_68888-68973(-) mir-142

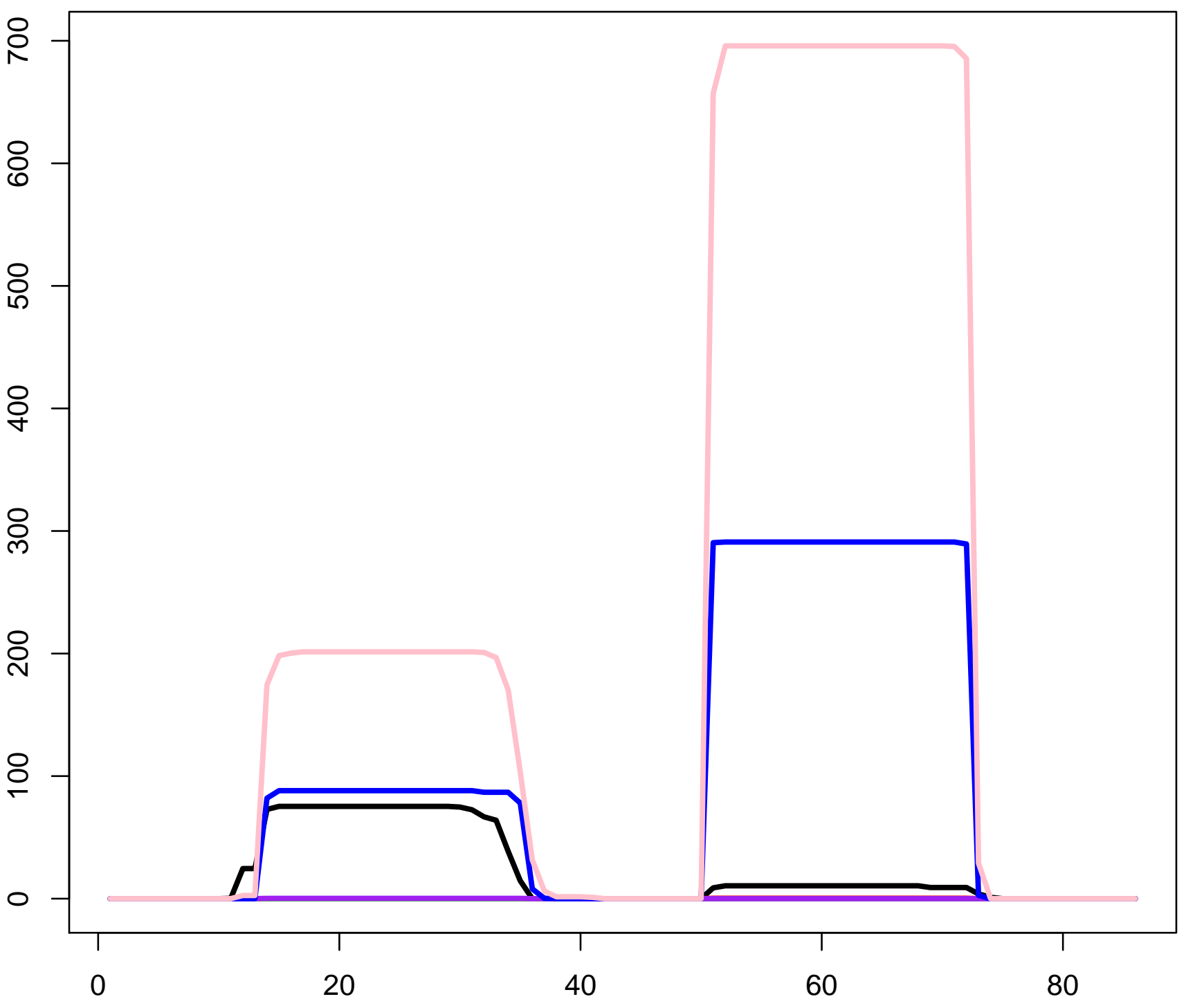

Egg $\rightleftharpoons$ St. 7-8 $\rightleftharpoons$ St. $12=$ St 18-20 $\rightleftharpoons$ St. 34-36 St. 56 
Scaffold95135_67052-67137(+) mir-146a

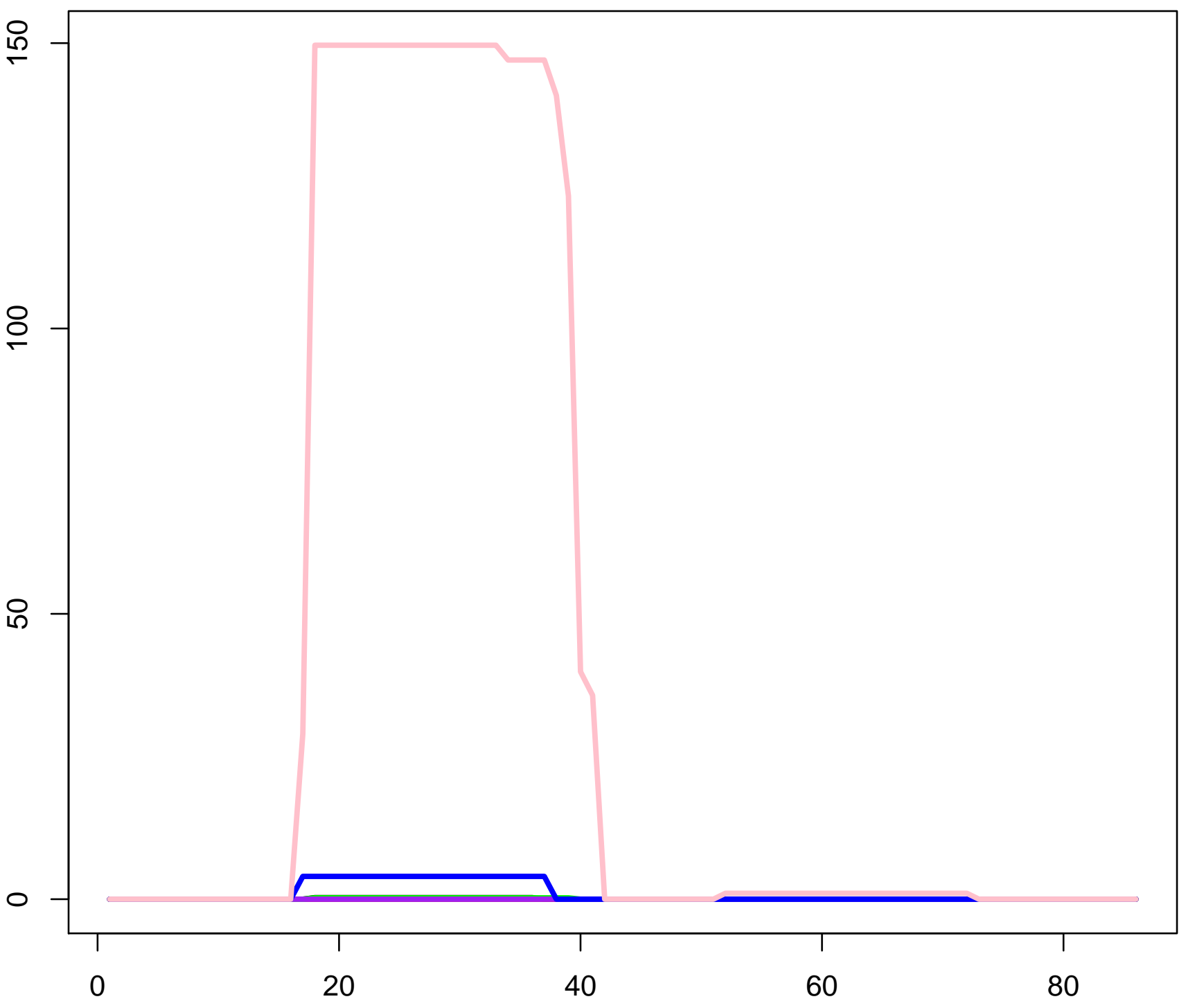


Scaffold95441_1909553-1909636(+) mir-29a-1

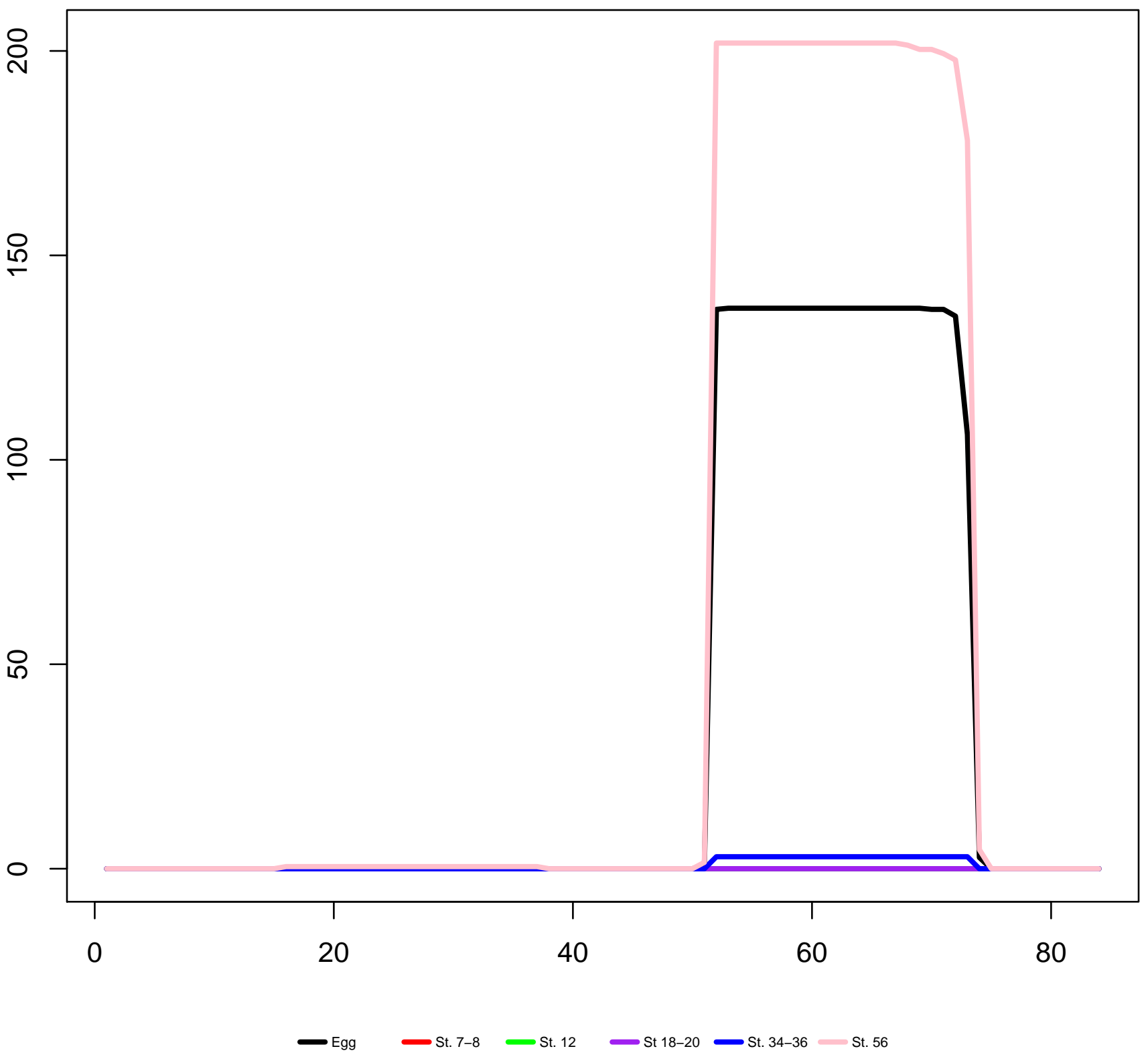


